\title{
Low-Voltage Electrowetting on Dielectrics Integrated and Investigated with Electrical Impedance Spectroscopy (LV-EWOD-EIS)
}

\author{
Dissertation \\ for the award of the degree \\ "Doctor rerum naturalium" \\ of the Georg-August-Universität Göttingen \\ within the doctoral program Chemistry \\ of the Georg-August-University School of Science (GAUSS)
}

submitted by

Yingjia Li

from Shanghai / China

Göttingen 2018 



\section{Thesis Committee}

Prof. Dr. Andreas Janshoff

Institute of Physical Chemistry, Georg-August-University Göttingen

Prof. Dr. Philipp Vana

Institute of Physical Chemistry, Georg-August-University Göttingen

Dr. Brian Cahill

Junior Research Group, Institute for Bioprocessing and Analytical Measurement Techniques

\section{Member of the Examination Board}

$1^{\text {st }}$ Referee: Prof. Dr. Andreas Janshoff

Institute of Physical Chemistry, Georg-August-University Göttingen

$2^{\text {nd }}$ Referee: Prof. Dr. Philipp Vana

Institute of Physical Chemistry, Georg-August-University Göttingen

\section{Further members of the Examination Board}

Dr. Brian Cahill

Junior Research Group, Institute for Bioprocessing and Analytical Measurement Techniques

Prof. Dr. Sarah Köster

Institute for X-Ray Physics, Georg-August-University Göttingen

Dr. Oliver Bäumchen

Dynamics of Complex Fluids, Max Planck Institute for Dynamics and Self-Organization

Dr. Thomas Burg

Biological Micro- and Nanotechnology, Max Planck Institute for Biophysical Chemistry

Date of oral examination: 07. 08. 2018 


\section{Declaration}

I, Yingjia Li, hereby declare that my doctoral thesis entitled "Low-Voltage Electrowetting on

Dielectrics Integrated and Investigated with Electrical Impedance Spectroscopy (LV-EWODEIS)" has been written independently with no other sources and aids than quoted.

Heiligenstadt, 2018

Yingjia Li 
Meiner Familie

„Die eigentliche Entdeckung besteht nicht darin, Neuland zu finden, sondern mit neuen Augen zu sehen.“

Marcel Proust 


\section{ABStRACT}

Electrowetting on dielectrics (EWOD) is an electrically controllable wetting effect, that has been applied in diverse fields including optics, displays and lab on a chip systems. However, the further development of EWOD applications and the online electrical characterization of EWOD are hindered by one major bottleneck, the need for high operating voltages. Due to the importance of overcoming this critical obstacle and better understanding the EWOD effect, a low-voltage EWOD electrode was developed in this thesis. It is based on a dielectric bilayer consisting of an anodic tantalum pentoxide $\left(\mathrm{Ta}_{2} \mathrm{O}_{5}\right)$ thin film with a high relative permittivity and a self-assembled hydrophobic silane monolayer. By means of this novel EWOD electrode, low-voltage EWOD was achieved. It shows a low voltage threshold of $2 \mathrm{~V}$ that enabled the integration of EWOD with electrical impedance spectroscopy (EIS). A further association with a high-speed imaging instrument facilitated the simultaneous EWOD excitation, optical imaging and impedimetric measurement using either frequency-domain EIS or dynamic EIS. This paved the way to online investigate the frequency dependence of EWOD on the application of alternating voltages as well as the non-linearity and dynamics of EWOD and dewetting. The EWOD configuration is an aqueous electrolyte droplet of $\mu \mathrm{L}$ volume on the low-voltage EWOD electrode in oil as the ambient medium. Between the droplet and the electrode, an oil layer of $\mathrm{nm}$ thickness is entrapped. Its thickness was determined during EWOD and dewetting. The frequency dependence of EWOD was studied with the focus on the Young-Lippmann equation, the fundamental equation of electrowetting theory, and its adaptions to account for the application of alternating voltages. The frequency dependence of the three EWOD-related variables in the equation was studied at various measurement conditions (applied voltage and salt concentration). The EWOD efficiency on the application of alternating voltages was assessed by a correlation coefficient introduced in the equation. Moreover, the non-linearity of EWOD and dewetting was characterized by the hysteresis in the deformation of the droplet and that of the entrapped oil layer. The characteristic time for the droplet deformation was determined to evaluate the dynamics of EWOD and dewetting. For the first time, both non-linearity and dynamics of EWOD and dewetting on the novel lowvoltage EWOD electrode were studied with dynamic EIS with the focus on the conversion efficiency of the electric potential energy in the deformation processes. 


\section{ZUSAMMENFASSUNG}

Die Elektrobenetzung auf Dielektrika („Electrowetting On Dielectrics“ - EWOD) ist ein elektrisch steuerbarer Benetzungseffekt, der seine Anwendung in verschiedenen Gebieten findet, von Optik, über Bildschirme bis hin zu Lab-on-a-Chip-Systemen. Allerdings werden die Weiterentwicklung der EWOD-basierten Anwendungen und die elektrische OnlineUntersuchungen der EWOD deutlich erschwert, weil hohe Betriebsspannungen für die EWOD notwendig sind. Aufgrund der Bedeutung der Überwindung der hohen Betriebsspannungen und des besseren Verständnisses des EWOD-Effektes wurde im Rahmen dieser Arbeit eine Niedrigspannungs-EWOD-Elektrode entwickelt. Sie basiert auf einer dielektrischen Doppelschicht aus einer anodischen Tantalpentoxid - $\left(\mathrm{Ta}_{2} \mathrm{O}_{5}\right)$ - Dünnschicht mit einer hohen Dielektrizitätskonstante und einer selbst-assemblierten hydrophoben Silan-Monoschicht. Diese neuartige EWOD-Elektrode ist als Niedrigspannungs-Elektrode geeignet. Sie weist eine niedrige Schwellenspannung von $2 \mathrm{~V}$ auf, die ermöglichte, den EWOD-Effekt mit elektrischer Impedanzspektroskopie (EIS) zu kombinieren. Die Verbindung mit einem bildgebenden Instrument mit einer hohen Aufnahmegeschwindigkeit ermöglichte die zeitgleiche Durchführung verschiedener Prozesse: EWOD-Anregung, optischer Bildaufnahme und Impedanzmessung mittels EIS bei Frequenzvariation oder dynamischer EIS. Damit war es möglich, eine Online-Untersuchung der Frequenzabhängigkeit der EWOD bei Anwendung von Wechselspannungen sowie der Nichtlinearität und Dynamik der Elektrobe- und Entnetzung durchzuführen. Die EWOD-Anordnung besteht aus einem wässrigen Elektrolyttropfen in einem Volumen von $\mu \mathrm{L}$ auf der Elektrode in Öl als Umgebungsmedium. Zwischen dem Tropfen und der Elektrode ist eine Ölschicht in einer Dicke von nm eingeschlossen. Deren Schichtdicke wurde während der Elektrobe- und Entnetzung bestimmt. Die Frequenzabhängigkeit der EWOD wurde mit dem Fokus auf der Young-LippmannGleichung, der grundlegenden Gleichung in der Theorie der Elektrobenetzung, und deren Anpassungen an Wechselspannungsanwendungen untersucht. Die Frequenzabhängigkeit der drei EWOD-relevanten Variablen in der Gleichung wurde unter verschiedenen Messbedingungen (angewendete Spannung und Salzkonzentration) untersucht. Die EWODEffizienz bei den Wechselspannungsanwendungen wurde anhand eines in die Gleichung eingeführten Korrelationskoeffizienten ausgewertet. Zudem wurde die Nichtlinearität der Elektrobe- und Entnetzung durch die Hysteresen in der Verformung des Tropfens und der eingeschlossenen Ölschicht charakterisiert. Die charakteristische Zeitkonstante der Tropfenverformung wurde ermittelt, um die Dynamik der Elektrobe- und Entnetzung zu evaluieren. Zum ersten Mal wurden sowohl die Nichtlinearität als auch die Dynamik der Elektrobe- und Entnetzung der neuartigen EWOD-Elektrode mittels dynamischer EIS mit dem Schwerpunkt auf dem Umwandlungswirkungsgrad der elektrischen Potentialenergie in den Verformungs-prozessen untersucht. 


\section{CONTENT}

1. Introduction .1

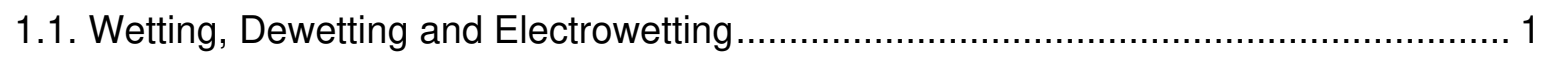

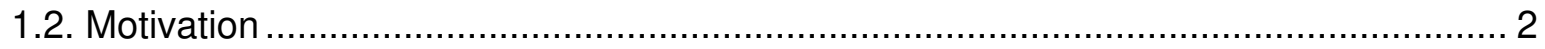

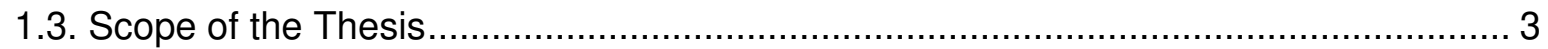

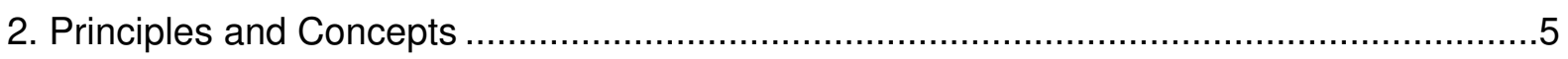

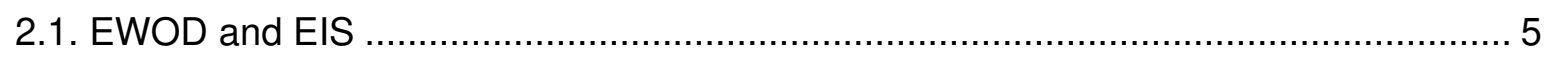

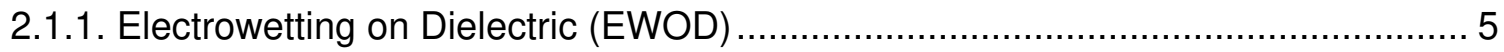

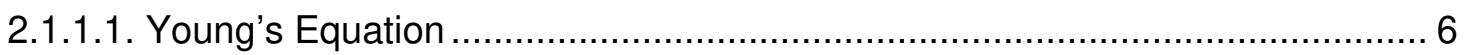

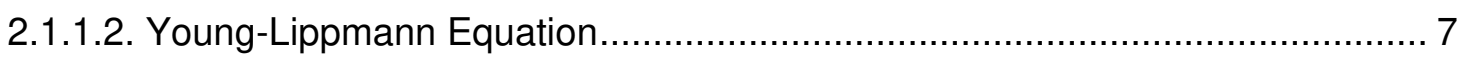

2.1.1.3. Derivation of Young-Lippmann Equation ................................................. 7

2.1.2. Electrical Impedance Spectroscopy (EIS) ................................................ 10

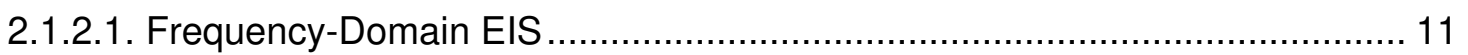

2.1.2.2. Time-Domain EIS and Dynamic EIS .................................................. 14

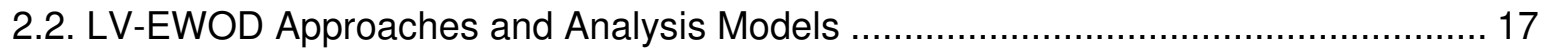

2.2.1. Low-Voltage Electrowetting on Dielectrics (LV-EWOD) …............................... 18

2.2.1.1. LV-EWOD Electrode Concept ................................................................ 18

2.2.1.2. Material Selection and Fabrication Methods for Dielectric Layers ................ 18

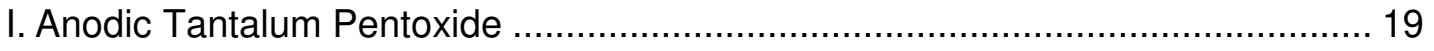

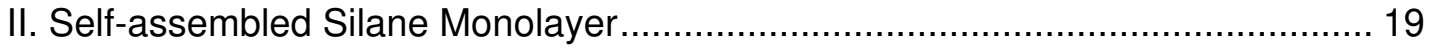

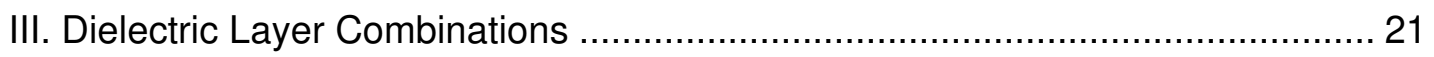

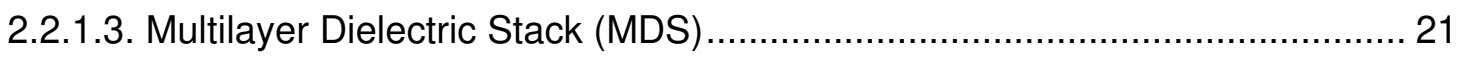

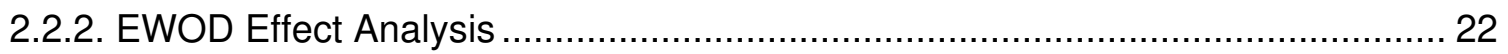

2.2.2.1. Equivalent Circuit Models ..................................................................... 22

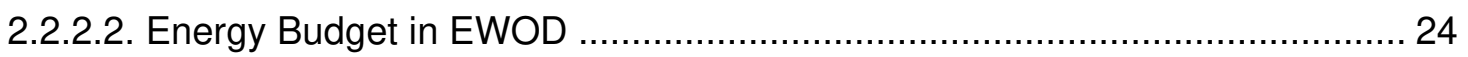

2.2.2.3. Young-Lippmann Equation for Alternating Voltage Applications................... 25

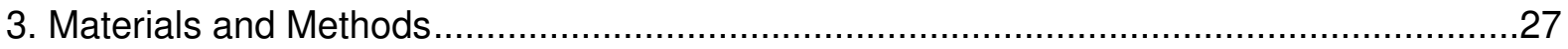

3.1. Fabrication of Low-Voltage (LV) EWOD Electrodes............................................ 27

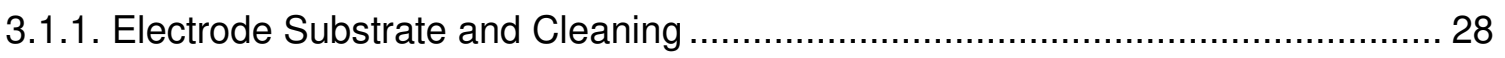

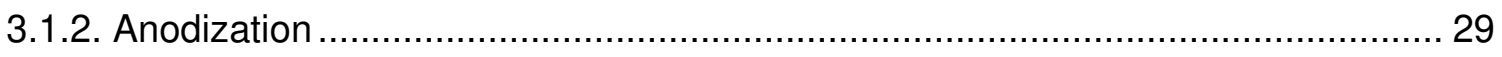

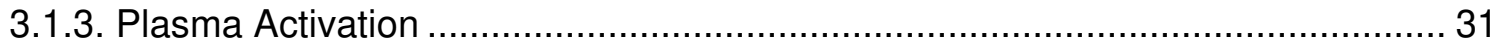




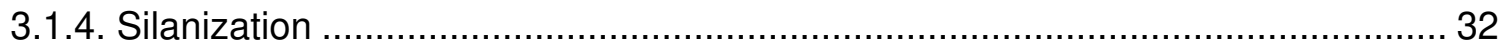

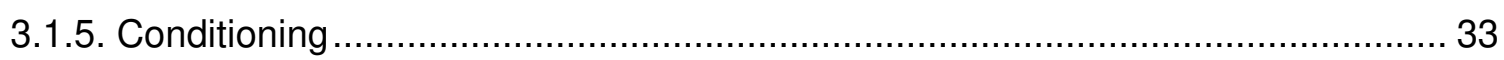

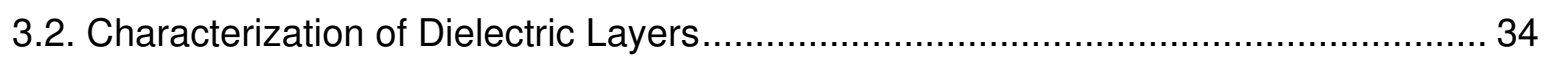

3.2.1. Electrical Impedance Spectroscopy: Capacitance......................................... 34

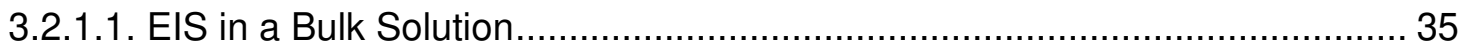

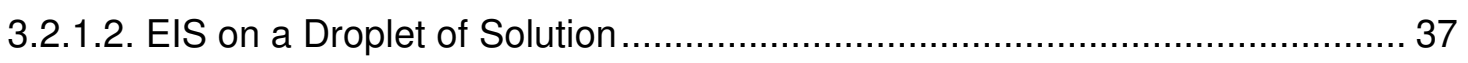

3.2.2. Cyclic Voltammetry: Capacitive Behavior ................................................... 39

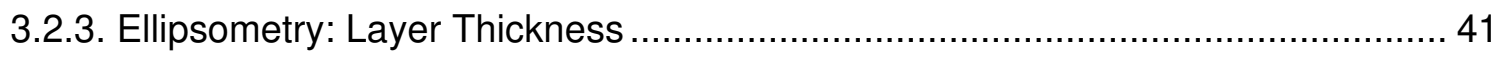

3.2.4. Atomic Force Microscopy: Surface Topology and Roughness .......................... 43

3.2.5. Contact Angle Determination: Surface Hydrophobicity.................................... 44

3.2.6. Measurement of Contact Angle Hysteresis: Surface Friction ............................ 46

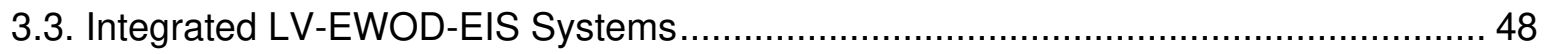

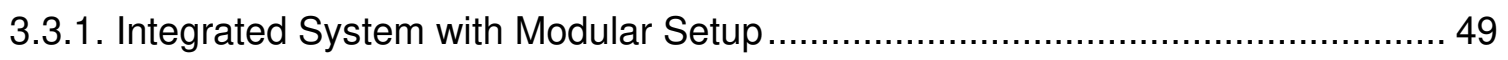

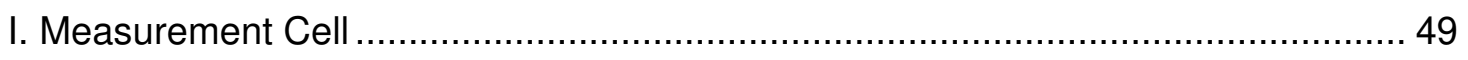

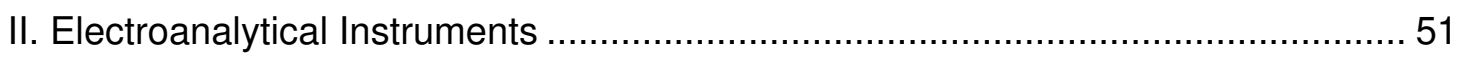

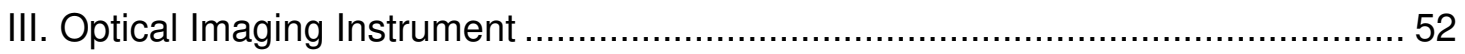

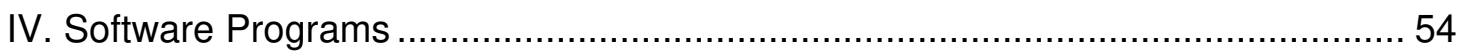

3.3.2. LV-EWOD with Frequency-Domain EIS …............................................... 55

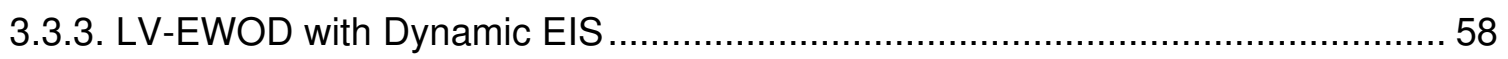

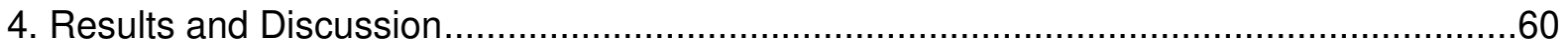

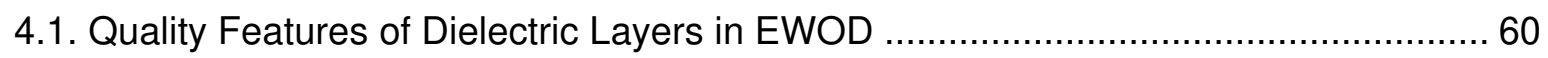

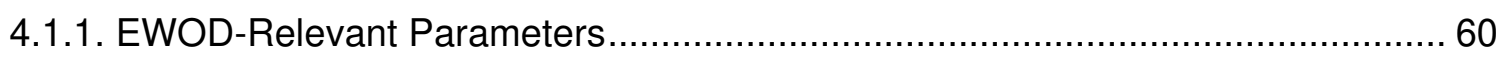

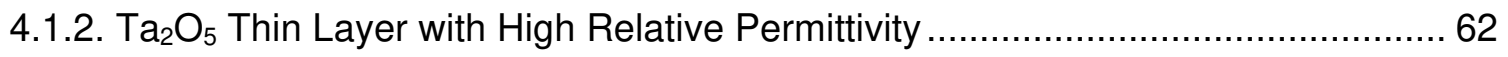

4.1.2.1. Homogeneous Smooth Hydrophilic $\mathrm{Ta}_{2} \mathrm{O}_{5}$ Surface .................................... 62

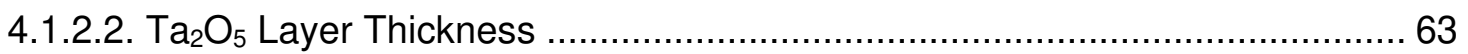

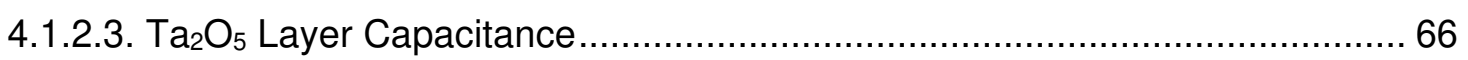

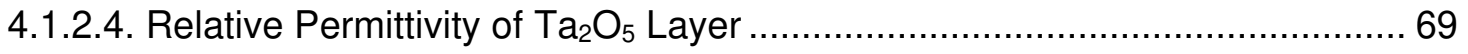

4.1.3. ODTS Monolayer with Hydrophobic Smooth Surface ....................................... 71

4.1.3.1. Topology and Hydrophobicity of ODTS Surface ...................................... 71

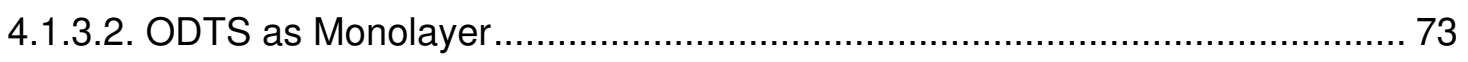

4.1.3.3. Capacitance of $\mathrm{Ta}_{2} \mathrm{O}_{5}$-ODTS-Bilayer and of ODTS Layer ........................... 74

4.1.3.4. Relative Permittivity of ODTS Monolayer .................................................. 76 
4.1.4. Multilayer Dielectric Stack Including an Oil Layer ............................................ 77

4.1.4.1. Low Surface Friction in Oil Surrounding............................................. 77

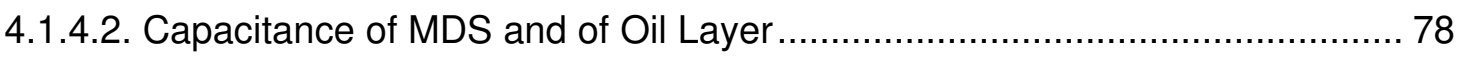

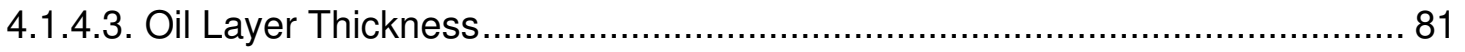

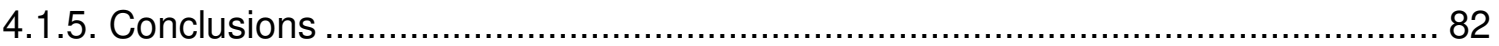

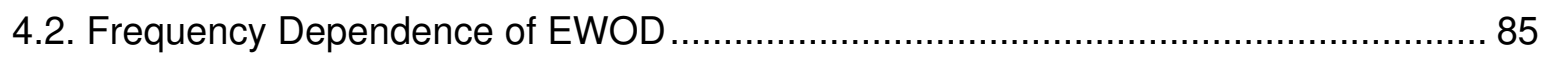

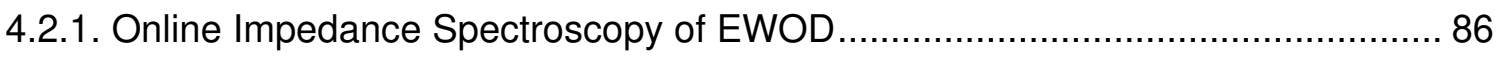

4.2.2. Voltage Drop on MDS Influenced by Frequency and Concentration ................... 88

4.2.3. MDS Capacitance Influenced by Oil Layer and Frequency ............................... 90

4.2.4. Contact Angle Influenced by Voltage and Frequnecy..................................... 91

4.2.5. Young-Lippmann Equation for AC Applications.............................................. 93

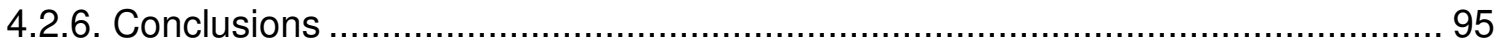

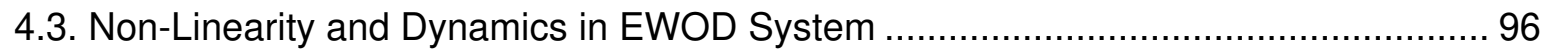

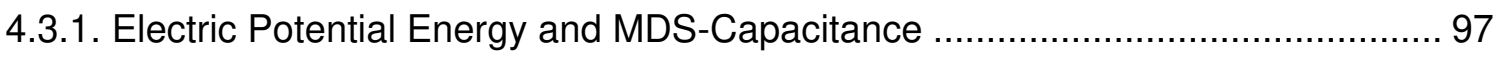

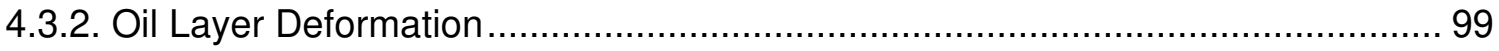

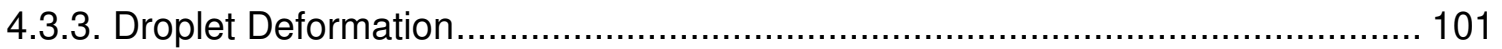

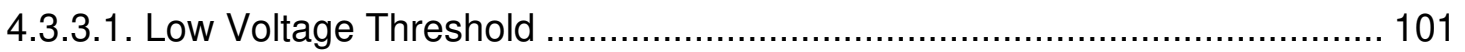

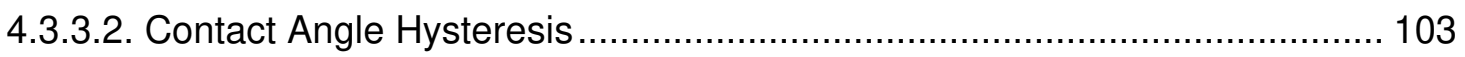

4.3.4. Contact Area Dynamics and Characteristic Time ........................................... 105

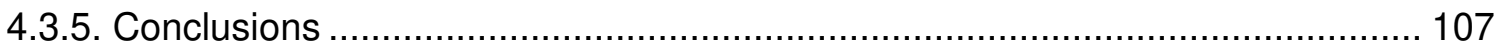

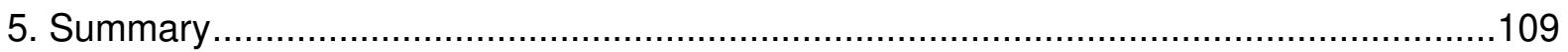

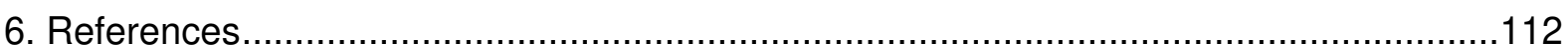

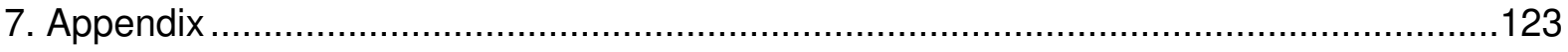

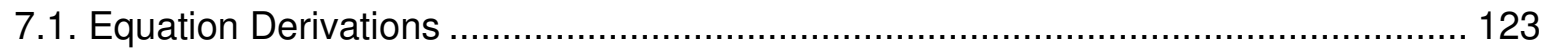

7.1.1. Equation for Assessment of Dynamic Relaxation Process .............................. 123

7.1.2. Root-Mean-Square Value of the Positive Alternating Voltages ........................ 125

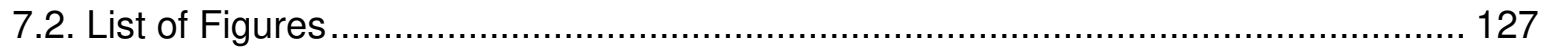

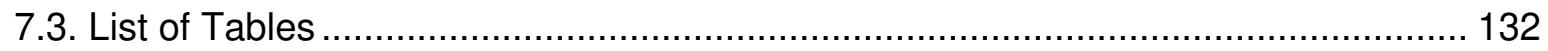

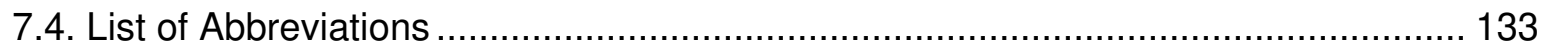

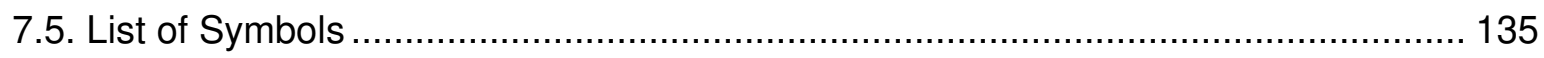

7.6. Circuit Diagram of Transimpedance Amplifier .................................................... 139

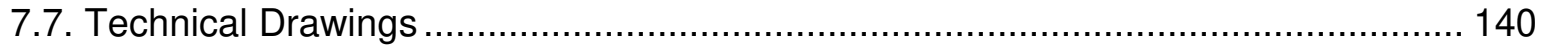


Publication

161

Selected Conference Contributions ... 161

Danksagung. 162 


\section{INTRODUCTION}

Wetting and dewetting are important and often encountered phenomena in nature and our daily life. Wetting is the spreading of a liquid on a solid surface and dewetting is the retraction of a fluid from a solid surface. Electrowetting, a voltage-dependent wetting effect, is the key subject of this doctoral research. In this opening section, the key development and applications of electrowetting are presented (Chapter 1.1) and followed by the motivation (Chapter 1.2) and the scope of this thesis (Chapter 1.3).

\subsection{Wetting, Dewetting and Electrowetting}

The "dew-collecting" Namib Desert beetles ${ }^{1-2}$ and the "self-cleaning" lotus leaves ${ }^{3-4}$ are the masterpieces of wetting and dewetting effect created by nature. In our daily life, wetting and dewetting of liquids on solid surfaces are phenomena of key importance as well. In inkjet printing $^{5}$, painting and cosmetics, wetting or spreading play an essential role; while dewetting and non-wetting are important in the dewatering from motorways, self-cleaning windows ${ }^{6}$ and anti-fouling paints ${ }^{7}$.

In these cases, the wetting and the dewetting effect are mainly determined by the surface chemistry and / or the surface topology. An alternative strategy to actively control and change the surface wettability is by applying electrical energy, known as electrowetting and further as electrowetting on dielectric (EWOD) ${ }^{8}$.

The foundation of electrowetting was laid by Lippmann ${ }^{9}$ in 1875 with his elucidation of electrocapillary effect. In 1993, Berge ${ }^{8}$ set another milestone with the introduction of electrowetting on dielectric (EWOD). In this modern concept, an aqueous liquid drop is placed on an electrode with a thin hydrophobic insulating coating; by applying a voltage across the insulating layer, electric potential energy gets stored in the insulating layer and makes the surface more easily wettable by a liquid drop.

EWOD is an interdisciplinary subject, where chemistry, physics and engineering meet and merge. EWOD behavior can be designed and programmed by means of multidisciplinary approaches, such as surface chemistry and interfacial interactions ${ }^{10}$, fluid hydrodynamics ${ }^{11}$, electrical stimuli and electrode architectures ${ }^{12-13}$.

The advantageous feature of being dynamic has opened a wide and diverse application field for EWOD, which has covered optics, displays and laboratory miniaturizations ${ }^{14}$.

The electrical control of drop shape parameters, such as curvature radius and wetting area, have led to EWOD finding application in liquid lenses ${ }^{15-16}$ with adjustable focus length and in electronic paper ${ }^{17-18}$ with inked droplets as individually addressable pixels. 
Moreover, the electrically driven deformation and translocation of droplets in microliter or nanoliter sizes have made EWOD the key foundation for the digital microfluidics ${ }^{14,}{ }^{19}$ with droplets as containers for reagents and samples being dispensed, moved, mixed, merged, and divided $^{20-22}$. This technique leads to diverse lab-on-a-chip microsystems ${ }^{23-24}$ in food-related or medical analytics.

\subsection{Motivation}

The EWOD-based applications face challenging bottlenecks in their development. One critical obstacle is the high operating voltages ${ }^{25}$. DC voltages about $25 \mathrm{~V}$ or alternating voltages around $50 \mathrm{~V}$ are required at least ${ }^{21,23,26 .}$

This high voltage demand is responsible for diverse drawbacks:

- It demands complicated electronics, materials with high electrical breakdown strength ${ }^{27}$ and thus results in high costs.

- High voltages can induce several side effects, such as irreversible electrowetting ${ }^{28}$, Ohmic warming and even electrolysis ${ }^{29}$.

- The need for high voltages is a hurdle for combining EWOD and electrical analysis techniques. Commercial electroanalytical equipment and most electronic components generally have a lower operating voltage limit than $25 \mathrm{~V}_{\mathrm{DC}}$ or $50 \mathrm{~V}_{\mathrm{AC}}$. The typical voltage range of potentiostats is around $\pm 10 \mathrm{~V}$. The power supply for operational amplifiers is normally within $\pm 15 \mathrm{~V}$.

- The high voltage application raises signal-output-errors in electrical measurements due to limited slew rate and driving capability ${ }^{30}$.

These high-voltage-related drawbacks hinder the EWOD effect from being thoroughly investigated by online electrical analysis. Furthermore, they are the limiting factors for combining EWOD actuation and electrical or electrochemical detection in developing integrated actuator - sensor systems.

The motivation to overcome these drawbacks and to better understand the EWOD effect has led to the main aims of this thesis:

- develop an approach to the low-voltage EWOD that facilitates the integration of EWOD with electrical impedance spectroscopy (EIS)

- investigate EWOD online with a focus on its frequency dependence in alternating voltage applications

- study the dynamics and non-linearity of EWOD and dewetting. 
EIS $^{31-32}$ is a non-invasive and widely used electrical analysis method. For this research, frequency-domain EIS is very suitable for investigating the frequency dependence of EWOD on application of alternating voltages. The high time resolution of dynamic EIS makes it an ideal measurement method for studying the dynamic and non-linear behavior of EWOD and dewetting.

Concerning EWOD applications using alternating voltages, the influence of frequency on EWOD has not yet been thoroughly studied. This is of key importance, since alternating voltages are more commonly applied than direct voltages especially in the digital microfluidic platforms ${ }^{14}$, 33, where EWOD-based actuators are integrated as key components. Moreover, using alternating voltages can greatly benefit EWOD since the voltage alternation can reduce the contact angle hysteresis and can thus ease the droplet motion and further enhance the EWOD effect. ${ }^{34}$

The dynamic behavior of EWOD and dewetting directly affects the actuation rate, which is a key performance feature of almost all EWOD applications in optics, displays and lab-on-a-chip systems ${ }^{14}$. The typical assessment of dynamics involves merely the optical detection of droplet deformation or translocation ${ }^{26}$. However, the dynamic behavior has not been studied with a further respect to the change of the electrical potential energy stored in the dielectric layer, which originates the EWOD and the dewetting. This is highly important to understand the dynamics and non-linearity of EWOD and the dewetting.

\subsection{Scope of the Thesis}

First, a low-voltage EWOD-EIS system was developed to fulfill the aims of the thesis. The centerpiece of the developed system was a novel EWOD electrode that was specially designed to require low voltages for inducing EWOD effect. In the low-voltage EWOD electrode, a dielectric bilayer coating was optimized according to the Young-Lippmann equation, the basis of the electrowetting theory. The dielectric bilayer was fabricated with the appropriately selected materials, tantalum pentoxide and silane, by electrochemical oxidation and by selfassembled monolayer deposition respectively.

With respect to the common EWOD applications in a surrounding oil ${ }^{35}$, the EWOD system in this study was configured as an aqueous $\mu \mathrm{L}$-droplet on the EWOD electrode in oil. Between the droplet and the electrode, a thin oil layer was entrapped as a fluid dielectric layer ${ }^{36}$, which is deformable during electrowetting. This oil layer and the two solid dielectric layers in the electrode formed a multilayer dielectric stack (MDS). Diverse surface, layer and material properties of each dielectric layer were characterized using atomic force microscopy (AFM), ellipsometry, contact angle measurements, cyclic voltammetry (CV) and EIS. The experimentally not easily accessible key parameters, such as relative permittivity and oil layer thickness in the nanometer range were indirectly determined via physical correlations. 
The low-voltage EWOD electrode was integrated in a low-voltage EWOD-EIS (LV-EWOD-EIS) system with associated high-speed optical imaging instrument in a modular setup. It enabled the online EWOD investigation with simultaneous EWOD excitation, EIS measurement and optical detection. For the EIS analysis, the object under study was described electrically using an equivalent circuit model and compared with a low-pass filter from electrical engineering.

Second, the study focused on the frequency dependence of EWOD, the efficiency of EWOD on application of alternating voltages. The EWOD excitation was integrated with frequencydomain EIS by using a single sinusoidal voltage signal with varied frequency and voltage. For droplets with salt concentrations in the physiologically relevant range, the frequency influence on the EWOD effect was characterized by using three EWOD-related variables in the YoungLippmann equation as indicators. They were simultaneously determined during electrowetting from its impedimetric response recorded by the LV-EWOD-EIS system and from the droplet deformation traced by high-speed imaging. The basic Young-Lippmann equation was adapted for alternating voltage by specifying the effective voltage drop across the MDS and by introducing a correlation coefficient that serves as an assessment parameter for the EWOD efficiency.

Third, the non-linearity and the dynamics of EWOD and dewetting were studied with the focus on the origin of the EWOD effect, the electric potential energy stored in MDS per unit area. As a determining factor of the electrical potential energy, the MDS-capacitance, was determined by dynamic EIS using a staircase voltage signal integrated with square wave function. Using this signal with an ascending and a descending ramp, EWOD and dewetting were induced correspondingly. The non-linearity was characterized by the voltage threshold of EWOD and the hysteresis behavior between EWOD and dewetting. These features were analyzed on the deformation of the droplet and that of the oil layer between the droplet and electrode. The dynamic behaviour was investigated by determining the characteristic time of the droplet geometry change at each voltage jump in the staircase signal. The change of the droplet geometry, that is, its contact angle and contact area, was traced by high-speed imaging in parallel to dynamic EIS at high time resolution. The electrical energy conversion in the deformation of droplet and oil layer was assessed with respect to the possible dissipation. 


\section{PRINCIPLES AND CONCEPTS}

\subsection{EWOD and EIS}

In this section, Chapter 2.1.1 focuses on the understanding of EWOD (electrowetting on dielectric) effect with a view of its theoretical principles. Chapter 2.1.2 is devoted to the principles of EIS (electrical impedance spectroscopy) and the integration of EWOD with frequency-domain EIS and with dynamic EIS.

\subsubsection{Electrowetting on Dielectric (EWOD)}

The phenomenon of interest, EWOD, is a wetting effect induced by electrical energy. ${ }^{35,37}$ This means, the spreading of a liquid on a solid insulator surface in a fluid surrounding (air or immiscible liquid) can be enhanced electrically. A general EWOD system (Figure $2.1 \mathrm{a}$ ) consists of a conductive liquid droplet partially wetting a planar dielectric or insulating solid layer. The droplet and the solid layer are connected to a power supply. By applying a voltage (Figure $2.1 \mathrm{~b}$ ), electrical energy causes the droplet to further spread on the solid surface. The change of the wetting state can be observed as the enlargement of the liquid-solid contact area and as the reduction of the contact angle from $\boldsymbol{\theta}_{\mathbf{0}}$ to $\boldsymbol{\theta}_{\mathbf{u}}$ on the intersection of the solidliquid interface and the liquid-ambient medium interface.

(a)

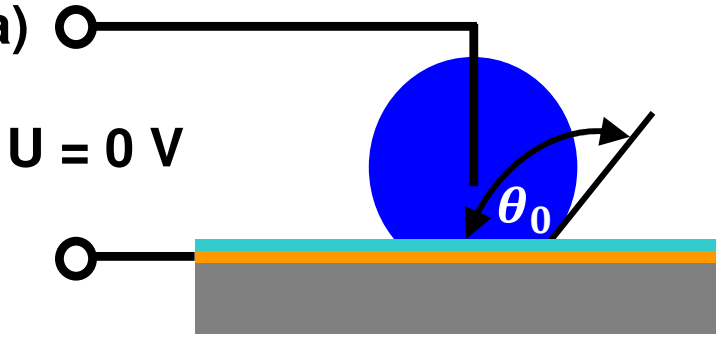

(b)

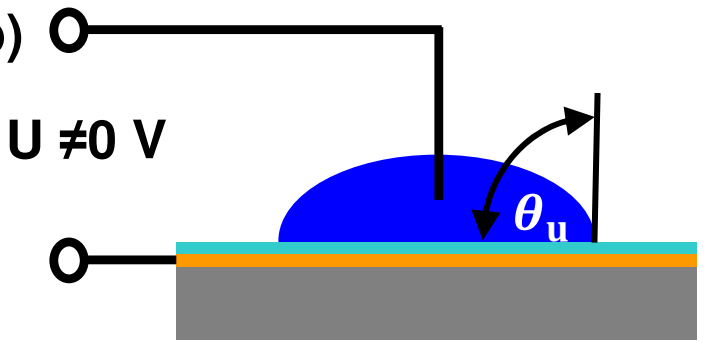

Figure 2.1. Electrowetting on dielectric (EWOD) effect demonstrated by a conductive droplet (dark blue) resting on a dielectric solid layer (bright blue) associated with an electrode layer (yellow) on a substrate (gray). (a) Under zero voltage: resting or dewetted state of the droplet with the static contact angle, $\theta_{0}$. (b) Under voltage: wetting state of the droplet with the voltagedependent contact angle, $\theta_{u}$.

In a reversible EWOD process, dewetting occurs upon switching off the supply voltage: the spread liquid contracts and dewets the solid surface with the contact angle increase and the contact area reduction. Ideally, the liquid returns to its resting state with the contact angle $\boldsymbol{\theta}_{\mathbf{0}}$ and the initial contact area from Figure 2.1 (b) to (a). 


\subsubsection{Young's Equation}

Without voltage application, the EWOD system is in a thermodynamic equilibrium state (Figure 2.2) with three interfacial tensions balanced on the contact line, the triple phase intersection.

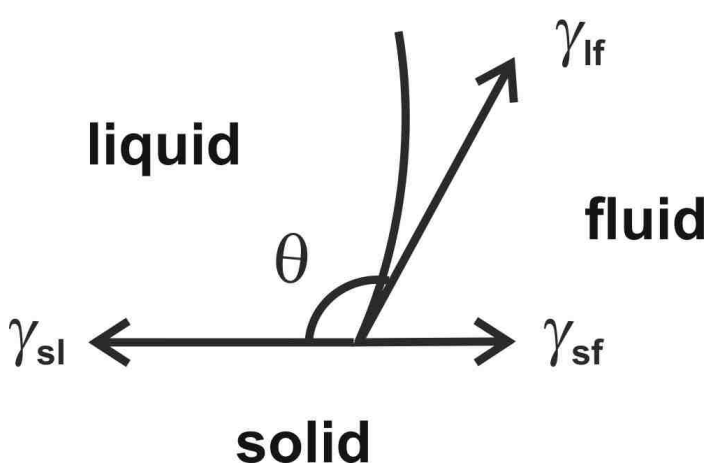

Figure 2.2. Equilibrium state of the three interfacial tensions on the triple-phase contact line. $\theta$ : Static contact angle. $\gamma_{\mathrm{sf}}$ : Solid-fluid interfacial tension. $\gamma_{\mathrm{sl}}$ : Solid-liquid interfacial tension. $\gamma_{\text {If }}$ : Liquid-fluid interfacial tension.

This balance results in the droplet contact angle $\left(\boldsymbol{\theta}_{\mathbf{0}}\right)$ and can be described with the Young's equation 38 :

$$
\cos \theta_{0}=\frac{\gamma_{\text {sf }}-\gamma_{\text {sl }}}{\gamma_{\text {lf }}}
$$

with the solid-fluid interfacial tension $\left(\gamma_{\mathbf{s f}}\right)$, the solid-liquid interfacial tension $\left(\gamma_{\mathbf{s l}}\right)$ and the liquidfluid interfacial tension $\left(\gamma_{\text {lf }}\right)$. The interfacial tension is defined as the Gibbs free energy change upon the contact area change at constant temperature, pressure and without material exchange with the exterior. ${ }^{39-41}$

Young's equation is derived under the thermodynamic equilibrium condition, that the change of the Gibbs free energy equals zero. A detailed derivation can be found in the textbook by Professor Butt et al. ${ }^{39}$. To ensure the equilibrium condition and thus the validity of Young's equation, the surrounding fluid phase in the EWOD system should be saturated with the liquid. ${ }^{39}$ In this study, an oil with a negligible solubility in water was used as the ambient medium for an aqueous droplet. In comparison to a liquid-air system, such a liquid-liquid system is more widely used in the EWOD-based applications, such as liquid lenses ${ }^{15}$, EWOD displays ${ }^{17}$ and digital microfluidic platforms ${ }^{14}$.

Moreover, Young's equation is the theoretical foundation for the contact angle measurement to investigate the surface hydrophobicity ${ }^{39,41-43}$. In this study, the contact angle measurement was implemented (i) with a commercial device to characterize the fabricated dielectric layers in the EWOD electrode (Chapter 3.2.5, 4.1.3.1, 4.1.4.1) and (ii) with an integrated EWOD-EIS system (Chapter 3.3.1) to analyze the droplet geometry at its resting state as well as its deformed state during EWOD and dewetting (Chapter 4.2.4, 4.3.3). 


\subsubsection{Young-Lippmann Equation}

The EWOD effect can be quantitatively described by the electrowetting equation, namely Young-Lippmann equation (Eq. 2.2). It expresses how the contact angle of the droplet changes with the applied voltage:

$$
\cos \theta_{\mathrm{u}}-\cos \theta_{0}=\frac{1}{2 \gamma_{\mathrm{lf}}} \cdot \frac{C}{A} \cdot U^{2}
$$

Here, $\boldsymbol{\theta}_{\mathbf{u}}$ and $\boldsymbol{\theta}_{\mathbf{0}}$ stand for the contact angles of a droplet with and without applied voltage respectively. $\gamma_{\text {If }}$ is the interfacial tension between the droplet liquid and its fluid surrounding. $\boldsymbol{C} / \boldsymbol{A}$ means the capacitance per unit area of the droplet-electrode interface. $\boldsymbol{U}$ is the voltage drop across the droplet-electrode interface.

In this doctoral research, the Young-Lippmann equation is the central theoretical foundation. It serves as a bridge between the EWOD-induced change of the droplet geometry $(\boldsymbol{\theta}$ and $\boldsymbol{A})$ and the EWOD-required electric potential energy $\left(\frac{1}{2} \cdot \frac{C}{A} \cdot U^{2}\right.$, Eq. 2.9), which correlates with the dielectric property of the insulating layer $(\boldsymbol{C} / \boldsymbol{A})$ as well as the applied voltage $(\boldsymbol{U})$. The droplet geometry parameters can be studied by optical imaging (Chapter 3.3.1); in parallel, the capacitive and electric parameters can be investigated by online EIS of EWOD (Chapter 3.3.2, 3.3.3) at diverse conditions. Based on Young-Lippmann equation and its modification adapted to the study conditions (Chapter 2.2.2.2), this research aims to provide a better understanding of the EWOD effect.

\subsubsection{Derivation of Young-Lippmann Equation}

As foundation of the electrowetting theory, the Young-Lippmann equation can be derived through diverse approaches, such as the interfacial thermodynamic approach ${ }^{35,37}$, the energy minimization approach ${ }^{8,35}$ and the electromechanical approach ${ }^{11,44-46}$. The first approach is based on Lippmann's equation (Eq. 2.5) and Young's equation (Eq. 2.1) concerning the interfacial thermodynamics. The second approach, according to $\mathrm{Berg}^{8}$, focuses on minimizing the Gibb's free energy of the droplet in an EWOD setup, which is contributed by the interfacial energies and by the electrostatic energy. The third approach has been developed by Jones et al. ${ }^{11,44-46}$ from a physical mechanical view by considering the electric-field-induced forces $^{47}$ that affect the liquid droplet.

Here, the Young-Lippmann equation is derived according to the first approach for a typical EWOD setup (Figure 2.1) with a dielectric layer as the defined droplet-electrode-interface. This typical EWOD setup represents the experimental setup in this study (Chapter 3.3.1). Moreover, this classic interfacial thermodynamic derivation is viewed with respect to the energy balance following the second approach.

Upon the voltage application in the EWOD system (Figure 2.1), an electric field induces charge accumulation on the droplet-electrode interface, more specifically, on the droplet-dielectric layer interface and on the dielectric layer-electrode interface. 
The dielectric layer can thus be considered as a capacitor according to the capacitor definition ${ }^{48}$. Its capacitance $(\boldsymbol{C})$ can be expressed as

$$
\boldsymbol{C}=\frac{Q}{\boldsymbol{U}}
$$

with the charge $(\boldsymbol{Q})$ on one interface, e.g. the dielectric layer-droplet (solid-liquid) interface and the voltage between the two interfaces $(\boldsymbol{U})$.

The charge density on this solid-liquid interface $\left(\boldsymbol{\rho}_{\mathrm{sl}}\right)$ is obtained as

$$
\rho_{\mathrm{sl}}=\frac{C}{A} \cdot U
$$

with reference to the area of the solid-liquid interface $(\boldsymbol{A})$.

The accumulated charge on the solid-liquid interface leads to the reduction of its interfacial tension. This effect was firstly explained by Lippmann ${ }^{9}$ in his investigation into electrocapillarity with an electrolyte directly contacted to a metal (mercury).' In his setup, the electric double layer adjacent to the electrolyte-metal interface works as the capacitor. According to Lippmann, the correlation of the interfacial tension $\left(\gamma_{\mathbf{s l}}\right)$, the charge density $\left(\boldsymbol{\rho}_{\mathbf{s l}}\right)$ on the solid-liquid interface and the voltage $(\boldsymbol{U})$ across the interface can be formulated as below:

$$
\left(\frac{\partial \gamma_{s l}}{\partial U}\right)_{\mu}=-\rho_{s l}
$$

Concerning the definition of chemical potential ${ }^{49}, \boldsymbol{\mu}=\left(\frac{\partial G}{\partial \boldsymbol{n}_{\boldsymbol{a}}}\right)_{\boldsymbol{p}, \boldsymbol{\Theta}}$, the boundary condition of the Lippmann's equation is implied to be the constant temperature, pressure and no material exchange with the exterior.

By combining Eq. 2.4 with Eq. 2.5, the reduction of the solid-liquid interfacial tension $\left(\boldsymbol{d} \gamma_{\mathrm{sl}}\right)$ can be described as:

$$
d \gamma_{\mathrm{sl}}=-\frac{C}{A} \cdot U \cdot d U
$$

The integral of Eq. 2.6 leads to the voltage dependent solid-liquid interfacial tension $\left(\boldsymbol{\gamma}_{\mathbf{s}(\boldsymbol{U})}\right)$ :

$$
\gamma_{\mathrm{sl}(U)}=\gamma_{\mathrm{sl}(0)}-\frac{1}{2} \cdot \frac{C}{A} \cdot U^{2}
$$

\footnotetext{
I The original work from Lippmann was written in French. A translation in English is provided in the review article: Mugele, F.; Baret, J.-C. Electrowetting: from basics to applications. J. Phys.: Condens. Matter 2005, 17, R705-R774.
} 
Moreover, this correlation can be viewed from the energy balance aspect.

By considering the interfacial dielectric layer as a capacitor with a capacitance of $\boldsymbol{C}$, its stored electric potential energy ${ }^{48}\left(\boldsymbol{E}_{\mathrm{el}}\right)$ under a voltage of $\boldsymbol{U}$ can be expressed as

$$
E_{\mathrm{el}}=\frac{1}{2} \cdot C \cdot U^{2}
$$

The corresponding areal value can be calculated with reference to the interfacial area as below:

$$
\frac{E_{\mathrm{el}}}{A}=\frac{1}{2} \cdot \frac{C}{A} \cdot U^{2}
$$

with the unit, $\mathrm{J} / \mathrm{m}^{2}$, equal to the unit of interfacial tension, $\mathrm{N} / \mathrm{m}$.

Based on this, Eq. 2.7 can be understand as the voltage-induced reduction of the solid-liquid interfacial tension $\left(\boldsymbol{\gamma}_{\mathbf{s l}(\mathbf{0})}-\boldsymbol{\gamma}_{\mathbf{s l}(\boldsymbol{U})}\right)$ equals the electric potential energy stored in the interfacial dielectric layer of unit area $\left(\boldsymbol{E}_{\mathrm{el}} / \boldsymbol{A}\right.$, Eq. 2.9).

This claim is supported by the derivation with the energy minimization approach ${ }^{8}$, 35: This electric potential energy dominates the electrostatic contribution to the EWOD effect. Another local contribution around the contact line, which arises from the stray capacitance and the fringe fields ${ }^{37,50}$, is negligible for the sufficiently large droplets as the case of this study.

Further on, the EWOD system at a constant voltage (Figure $2.1 \mathrm{~b}$ ) is at an equilibrium state of the three interfacial tensions: the voltage-dependent, reduced solid-liquid interfacial tension $\left(\gamma_{\mathbf{s l}(\boldsymbol{U})}\right)$ and the other two voltage-independent interfacial tensions $\left(\boldsymbol{\gamma}_{\mathbf{s f}}\right.$ and $\left.\boldsymbol{\gamma}_{\mathbf{l f}}\right)$. This equilibrium is reflected with the contact angle under voltage $\left(\boldsymbol{\theta}_{\mathbf{u}}\right)$ and can be described with the Young's equation:

$$
\cos \theta_{\mathrm{u}}=\frac{\gamma_{\mathrm{sf}}-\gamma_{\mathrm{sl}(U)}}{\gamma_{\mathrm{lf}}}
$$

By combining Eq. 2.1, Eq. 2.7 and Eq. 2.10, the Young-Lippmann equation (Eq. 2.2) is derived.

For the EWOD electrode with a planar structure, as the case in this study, the interfacial dielectric layer can be modeled as a parallel plate capacitor. Its capacitance per unit area $(\boldsymbol{C} / \boldsymbol{A})$ depends on the relative permittivity $\left(\boldsymbol{\varepsilon}_{\boldsymbol{d}}\right)$ of its material and its layer thickness $(\boldsymbol{d})$ as below:

$$
\frac{C}{A}=\frac{\varepsilon_{0} \varepsilon_{d}}{d}
$$

with $\varepsilon_{0}$ standing for the vacuum permittivity. 
By combining with Eq. 2.11, the Young-Lippmann equation (Eq. 2.2) has the following expression:

$$
\cos \theta_{u}-\cos \theta_{0}=\frac{\varepsilon_{0} \varepsilon_{d}}{2 \gamma_{l f} d} \cdot U^{2}
$$

Here, $\boldsymbol{U}$ is the applied voltage across the interfacial dielectric layer.

Concerning the aim of this study to induce the EWOD effect on application of low voltages, a further explanation of $\boldsymbol{U}$ is given below: In the differential equation of Lippmann (Eq. 2.5), the original $\boldsymbol{U}$ is the applied voltage minus the voltage required to compensate the potential of zero charge $\left(\boldsymbol{U}=\boldsymbol{U}_{\text {app }}-\boldsymbol{U}_{\text {pzc }}\right)$. In contrast to a metal surface, which was directly contacted to electrolyte in Lippmann's experiment, the surface of an insulating material should not induce the spontaneous charge accumulation without voltage application ${ }^{35}$. Moreover, in the integrated Lippmann equation, the potential of zero charge can be considered negligible, since it is even in case of a metal (in the sub- $\mathrm{mV}$ range) ${ }^{51}$ significantly lower than the normal applied voltage $(25-50 \mathrm{~V})^{21,23,26}$ by two to three magnitudes. Therefore, for the practical investigation of EWOD effect, the applied voltage is usually regarded as the same as $\boldsymbol{U}$.

In this study with low voltages, the resting state is defined at $\boldsymbol{U}=0 \mathrm{~V}$, each applied voltage is an increment to the zero voltage.

\subsubsection{Electrical Impedance Spectroscopy (EIS)}

EIS $^{32,52-53}$, electrical impedance spectroscopy, is a non-invasive electrical analysis method using AC signals for material and process characterization. Its application can be found in versatile fields: on-line monitoring of living cells ${ }^{54}$ in life science ${ }^{55}$, control of product quality and production processes ${ }^{56}$ in food industry and characterization of batteries ${ }^{57}$ and solar cells ${ }^{58}$ in energy sector.

As an important analysis method, EIS yields useful information about the electrical features of the object under measurement. Furthermore, diverse material and process parameters can be obtained from the EIS information through further analysis. For example, aqueous droplets in segmented flow can be online determined for their conductivity based on the EIS technique ${ }^{59}$.

Moreover, EIS has also been utilized together with EWOD as integrated sensor-actuators. The two complimentary technologies combined on one platform close the gap between the operation and the detection of fluids, which is advantageous or even demanded in diverse application fields. EIS in association with electrowetting actuation has led to active matrix EWOD devices, in which the presence and the size of droplets can be determined ${ }^{12}$. A digital microfluidic platform with integrated EIS and EWOD enables the automatic actuation of droplets, to characterize the droplet composition of particles and the measurement conditions ${ }^{60-61}$. 
In addition, EIS has been applied to study various EWOD arrangements concerning the droplet configuration. By using EIS technique, droplet contact area can be detected during electrowetting and its geometry can be further interrogated ${ }^{62-63}$.

The sections focus on the theoretical basics of the electrical impedance spectroscopy (EIS) with respect to its two measurement techniques: EIS in frequency domain (Chapter 2.1.2.1) and EIS in time domain (Chapter 2.1.2.2). In this study, the two EIS techniques are integrated with EWOD excitation to enable the online investigation into the frequency influence on the EWOD effect (Chapter 4.2) and the non-linear and dynamic processes in the EWOD system (Chapter 4.3).

\subsubsection{Frequency-Domain EIS}

The general principle of frequency-domain EIS is the measurement of the current response of the system under investigation to an alternating voltage signal applied as stimulus. The basic alternating voltage stimulus $(\boldsymbol{U})$, as illustrated in Figure 2.3 (red curve), is a sinusoidal function of time $(\boldsymbol{t})$. It can be expressed as:

$$
U=U_{\mathrm{pp} / 2} \cdot \sin \omega t
$$

where $\boldsymbol{U}_{\mathrm{pp} / \mathbf{2}}$ is the voltage amplitude and $\boldsymbol{\omega}$ is the angular frequency.

This voltage stimulus results in a current flowing through the system under investigation. The current response of a (pseudo)-linear system or a non-linear system at its steady state, is also a sinusoidal function as demonstrated in Figure 2.3 (blue curve). Due to the electrical properties of the components in the system under investigation, there may be a phase shift $(\boldsymbol{\phi})$ between the voltage stimulus and current response. This can be expressed as:

$$
I=I_{\mathrm{pp} / 2} \cdot \sin (\omega t+\phi)
$$

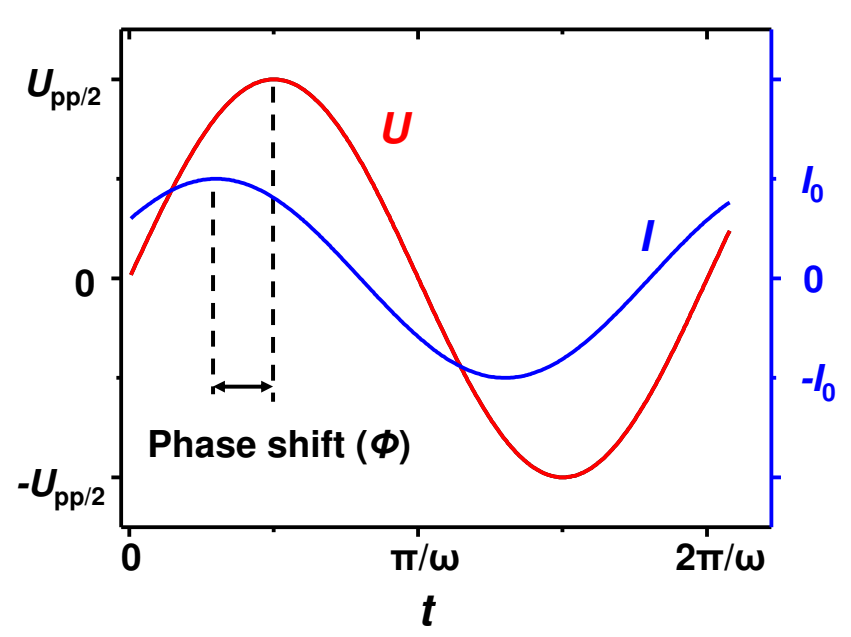

Figure 2.3. Measurement principle of EIS in frequency domain. $U$ : sinusoidal voltage stimulus (red), l: current response (blue), $\phi$ : phase shift between the voltage and current signals. 
In analogy to Ohm's law, the impedance $(Z)$ can be calculated and written in its polar form $\mathbf{a s}^{64}$ :

$$
Z=\frac{U}{I}=|Z| \cdot e^{j \phi}
$$

Here, $\boldsymbol{j}=\sqrt{-1}$ is the imaginary unit and $\boldsymbol{Z}$ is a complex impedance. $|\boldsymbol{Z}|$ is the impedance modulus, the magnitude of $\boldsymbol{Z}$.

With Euler's formula ${ }^{65}, e^{j x}=\cos x+j \cdot \sin x$, Eq. 2.15 can be transformed to:

$$
Z=|Z| \cdot \cos \phi+j \cdot|Z| \cdot \sin \phi=Z_{R e}+j \cdot Z_{I m}
$$

The complex impedance can be generally represented with the separated real $\left(\boldsymbol{Z}_{\boldsymbol{R} \boldsymbol{e}}\right)$ and imaginary $\left(\boldsymbol{Z}_{\boldsymbol{I m}}\right)$ parts ${ }^{64}$.

Concerning the impedimetric investigation of a physicochemical system or process, equivalent circuit models ${ }^{55,66}$ are commonly constructed to electrically describe the object under study. The basic physical components of equivalent circuit models are resistors and capacitors. For example, the electrochemical double layer (EDL) ${ }^{39,64}$ at the electrode-electrolyte interface is commonly modeled as a capacitor, while a bulk electrolyte is usually represented as a resistor. In biology, the cell membrane is usually simplified to be represented as a capacitor and the cytosol as a resistor ${ }^{67}$. In this study, a resistor and a capacitor in series connection is applied as the equivalent circuit model to electrically describe the EWOD system (Chapter 2.2.2.1).

The complex impedance of a resistor, a capacitor and a series $R C$ circuit are derived below.

First, by applying a sinusoidal voltage across a resistor, its current response is in phase with the applied voltage. By inserting $\boldsymbol{\phi}=\mathbf{0}$ in Eq. 2.16 and Eq. 2.15, $\boldsymbol{Z}$ is reduced to its real part and is determined by Ohm's law:

$$
Z=|Z| \cdot \cos \phi=Z_{R e}=\frac{U}{I}=R
$$

Thus, the impedance of a resistor possesses merely a real part, which is equal to the resistance $(\boldsymbol{R})$.

Second, by applying a sinusoidal voltage across a capacitor, its current response to the applied voltage has a lag of $\pi / 2$. Due to $\phi=-\pi / 2$, Eq. 2.16 is reduced to its imaginary part:

$$
Z=j \cdot|Z| \cdot \sin \phi=-j \cdot Z_{I m}
$$


Based on the definition of capacitance ( $\boldsymbol{C}=\frac{\boldsymbol{Q}}{\boldsymbol{U}}$, Eq. 2.3), that of current $\left(\boldsymbol{I}=\frac{\boldsymbol{d} \boldsymbol{Q}}{\boldsymbol{d} t}\right)$ and Eq. 2.15 at $\boldsymbol{\phi}=-\boldsymbol{\pi} / \mathbf{2}$, the impedance of a capacitor can be derived $\mathrm{as}^{64}$ :

$$
Z=-j \cdot \frac{1}{\omega C}=-j \cdot X_{\mathrm{c}}
$$

with $X_{\mathbf{c}}$ standing for the capacitive reactance $\left(X_{\mathbf{c}}=\frac{1}{\omega C}\right)^{48}$.

The combination of Eq. 2.18 and Eq. 2.19 yields:

$$
|Z|=Z_{I m}=X_{c}
$$

Hence, the impedance of a capacitor consists solely of an imaginary part, which is equal to the capacitive reactance.

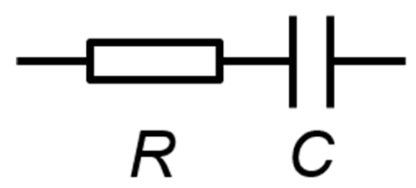

Figure 2.4. Series connection of a resistor and a capacitor.

Third, for an electric circuit consisting of a resistor and a capacitor in series (Figure 2.4), the application of the sinusoidal voltage across them follows Kirchhoff's law ${ }^{48}$ :

$$
\boldsymbol{U}=\boldsymbol{U}_{\boldsymbol{R}}+\boldsymbol{U}_{\boldsymbol{C}}
$$

The entire impedance is the sum of the impedance of the resistor (Eq. 2.18) and that of the capacitor (Eq. 2.19):

$$
\boldsymbol{Z}=\boldsymbol{R}-\boldsymbol{j} \cdot \boldsymbol{X}_{\mathbf{c}}
$$

The impedance modulus and the phase shift can be calculated as:

$$
\begin{gathered}
|Z|=\sqrt{R^{2}+X_{c}^{2}} \\
\phi=\tan ^{-1}\left(-\frac{X_{c}}{R}\right)=\tan ^{-1}\left(-\frac{1}{\omega R C}\right)
\end{gathered}
$$

In this study, impedance spectra are presented in Bode plots ${ }^{53,64}$, in which $\log |Z|$ and $\boldsymbol{\phi}$ are plotted against the logarithm of frequency $\left(f=\frac{\omega}{2 \pi}\right)$ respectively.

For a series $R C$ circuit, the phase shift is negative and varies between 0 and $-\pi / 2$; it indicates the balance between $R$ and $C$. A break-point frequency ${ }^{53}$ or critical frequency $\left(\omega_{c}=\frac{1}{R C} ; f_{c}=\frac{1}{2 \pi R C}\right)$ is defined for $\phi=\pi / 4$, where the resistive component and the capacitive component contribute equally to $|Z|$. 
This study applies frequency domain EIS for the online investigation of the frequency influence on the EWOD effect. The EWOD excitation and the simultaneous EIS measurements are achieved here with a single sinusoidal voltage signal. By applying a series of voltages and frequencies successively on the EWOD system with each condition maintaining for sufficient time, the system attains the steady-state for each impedance measurement (Chapter 3.3.2). The correlations derived in this chapter are involved in the data processing for compiling impedance spectra in Bode plots and in the data analysis for yielding further features of the EWOD system (Chapter 4.2).

\subsubsection{Time-Domain EIS and Dynamic EIS}

One of the most important excitation signals for time-domain EIS ${ }^{55}$ is the square wave. In this study, it is the basic signal involved in the dynamic EIS ${ }^{68}$ applied for investigation into the dynamics of EWOD effect. A detailed description about the dynamic EIS is provided further on. A square wave can be considered as a series of alternating positive and negative voltage steps (Figure 2.5).

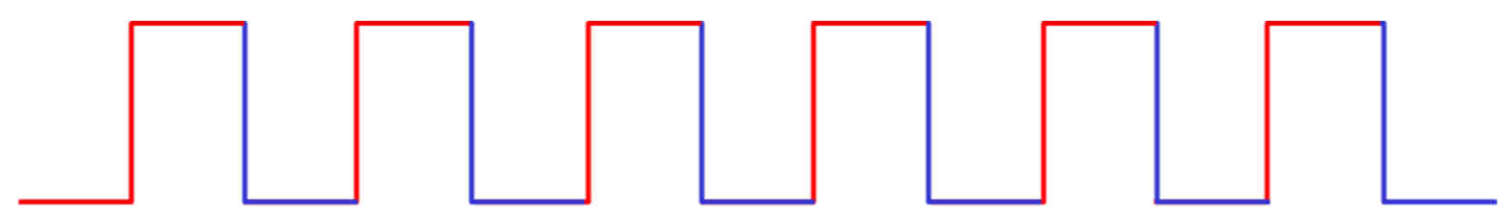

Figure 2.5. Square wave as a series of alternating positive (red) and negative (blue) voltage steps.

The basic principle of EIS in time domain is to apply a voltage step as electrical perturbation to the system under investigation and to analyze its current response ${ }^{64}$.

Upon applying a positive voltage step $(\boldsymbol{U})$ on a series $R C$ circuit (Figure 2.4), the current $(\boldsymbol{I})$ flowing through the circuit reaches first its maximal amplitude and immediately decays with the time $(\boldsymbol{t})$ as illustrated in Figure 2.6.

The current decay reflects the electrical relaxation behavior of the system under investigation and can be expressed as:

$$
I=\frac{U}{R} \cdot e^{-t / R C}
$$

Its derivation can be found in the textbook written by Bard and Faulkner ${ }^{64}$. The exponential function of the current decay contains diverse information: Its initial maximal amplitude correlates inversely with the resistance $(\boldsymbol{R})$ in the system. The time constant $(\boldsymbol{\tau})$ equals the product of $\boldsymbol{R}$ and $\boldsymbol{C}$ and is reciprocal to the corresponding critical frequency $\left(\boldsymbol{\omega}_{\boldsymbol{c}}\right)$ of EIS in frequency domain. At $\boldsymbol{t}=\boldsymbol{\tau}$, the current decreases to $0.37 \boldsymbol{U} / \boldsymbol{R}$. 


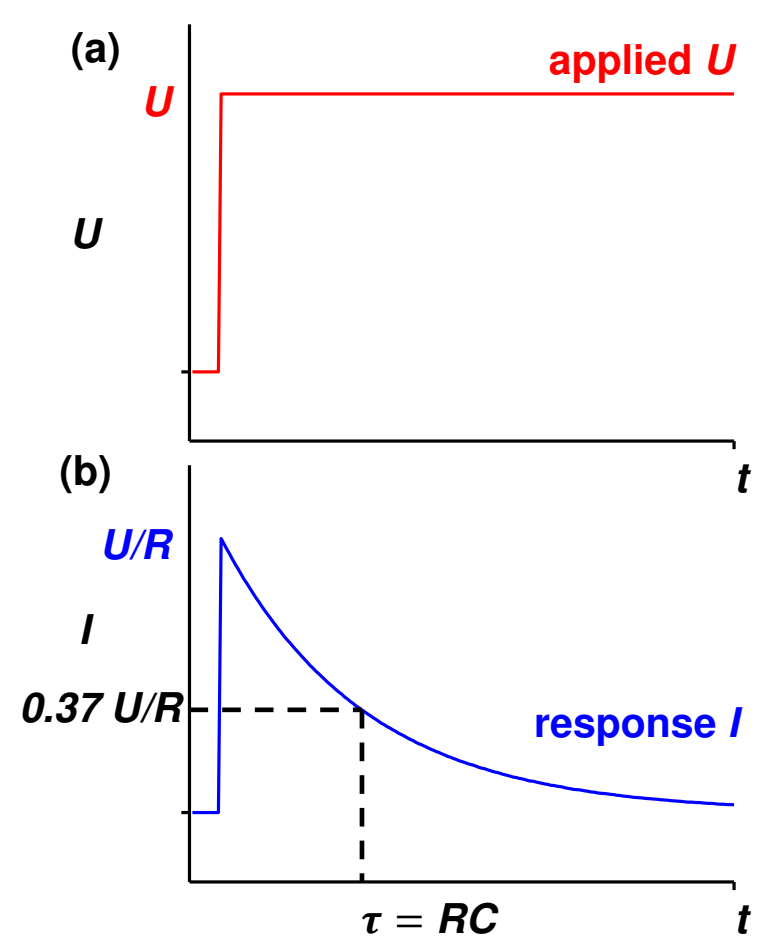

Figure 2.6. Basic principle of EIS in time domain demonstrated for a series $R C$ circuit. (a) A positive voltage step as electrical perturbation. (b) Current response with the time constant $(\tau)^{\prime \prime}$.

By applying a negative voltage step to the series $R C$ circuit, the current responses in the negative region in the identical manner: a maximal negative amplitude followed by an exponential decay towards zero. This current response yields the same impedimetric information as that under the application of a positive voltage.

Based on this, EIS measurements with a high repetition rate can be achieved by using a square voltage signal with a high frequency. This makes the EIS in time domain advantageous for rapid measurement requirements ${ }^{55}$. Moreover, the square wave is a typical broad bandwidth signal, ${ }^{69}$ which contains all odd harmonic signals. ${ }^{53}$ The impedimetric information related to a broad frequency range can be obtained with EIS in time domain in each single voltage step.

\footnotetext{
" Own representation based on Bard, A. J.; Faulkner, L. R. Electrochemical Methods: Fundamentals and Applications. 2 ed.; JOHN WILEY \& SONS, INC.: New York, Chichester, Weinheim, Brisbane, Singapore, Toronto, 2001, p. 16.
} 
In this study, the non-linear and dynamic processes in the EWOD system are investigated by dynamic EIS ${ }^{53,68}$. It combines the EIS in time domain using a high-frequency square signal with the EWOD excitation using a DC (direct current) staircase voltage signal (Figure 2.7).

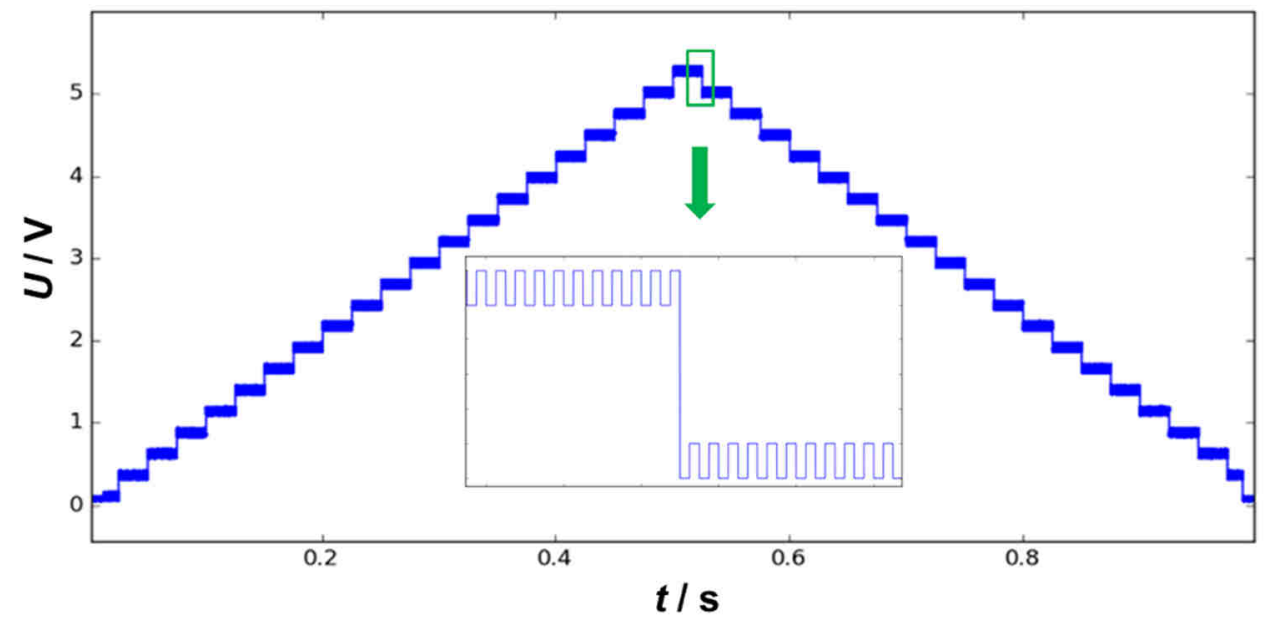

Figure 2.7. Dynamic EIS signal for investigation into the dynamic behavior of EWOD effect. Main panel: Staircase voltage signal for the EWOD excitation. Inset (enlargement of the green marked region): Square signal superposed in each DC voltage step for EIS measurement in time domain.

The staircase signal consists of an ascending ramp with increments of voltage offsets for EWOD excitation and a descending ramp with decrements of voltage offsets for controlled dewetting relaxation. At each step, the voltage offset is superposed with a square signal of high frequency for the rapid EIS measurement to trace the EWOD-system change during the wetting and dewetting process time-resolved.

Dynamic EIS is an appropriate method for characterization of time-varying and non-linear processes, such as the droplet deformation during EWOD and dewetting, for the following reasons ${ }^{68}$ : First, the EIS measurement period is shorter than the characteristic time of the EWOD-system change during wetting and dewetting process. Second, the small amplitude of the EIS excitation signal enables the EIS measurement within a linear or quasi-linear range although the superposed larger DC staircase signal inducing the non-linear EWOD effect. 


\subsection{LV-EWOD Approaches and Analysis Models}

Partial content in this chapter has been published in Li, Ying-Jia and Cahill, Brian P.,

"Frequency Dependence of Low-Voltage Electrowetting Investigated by Impedance

Spectroscopy”, Langmuir 2017, doi: 10.1021/acs.langmuir.7b03049.

This section is mainly devoted to the theoretical approach for the low-voltage electrowetting on dielectric (LV-EWOD) effect and the analysis model for the investigation with the electrical impedance spectroscopy (EIS). An overview of the development process and the diverse aspects concerning the online electrical investigation of EWOD effect is given in the following illustration.
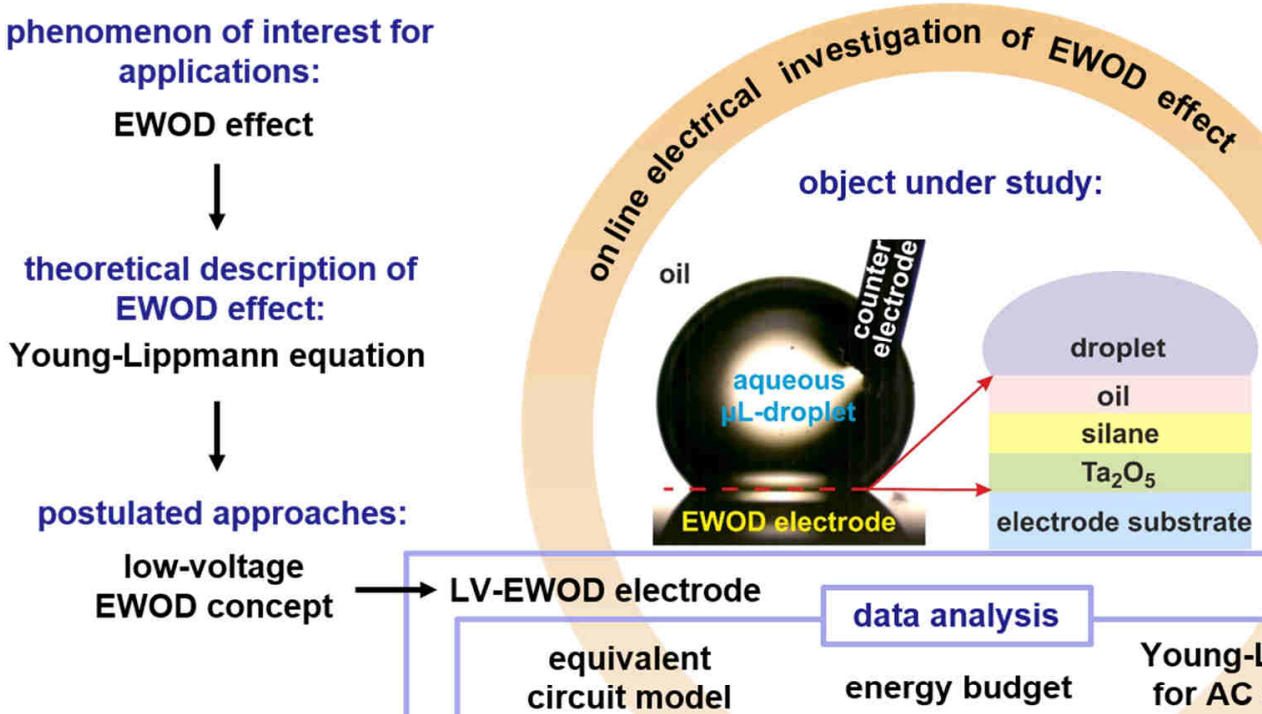

Young-Lippmann eq.
for AC applications circuit model

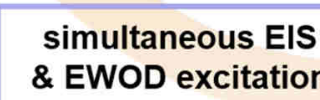

optical imaging

data acquisition

LV-EWOD-EIS system integrated with an imaging instrument

Figure 2.8. Overview of the development process and the diverse aspects concerning the online electrical investigation of EWOD effect.

As the fundamental equation describing the EWOD effect, the Young-Lippmann equation (Eq. 2.2) provides the foundation of Chapter 2.2.1 to develop the concept of low voltage EWOD and the approach for a LV-EWOD-electrode for the EWOD-EIS integration. In Chapter 2.2.2, an equivalent circuit model is postulated for the object under investigation in the EWOD system. An adapted Young-Lippmann equation is formulated for evaluating the EWOD effect in AC (alternating current) applications that accounts for the electric energy budget in the EWOD system. 


\subsubsection{Low-Voltage Electrowetting on Dielectrics (LV-EWOD)}

\subsubsection{LV-EWOD Electrode Concept}

Based on the Young-Lippmann equation (Eq. 2.2) and its transformation (Eq. 2.12),

$$
\cos \theta_{\mathrm{u}}-\cos \theta_{0}=\frac{C}{2 \gamma_{\mathrm{lf}} A} \cdot U^{2}=\frac{\varepsilon_{0} \varepsilon_{d}}{2 \gamma_{\mathrm{lf}} d} \cdot U^{2}
$$

the voltage demand for EWOD can be reduced through three approaches:

(i) Increase of the relative permittivity of the dielectric layers $\left(\boldsymbol{\varepsilon}_{\boldsymbol{d}}\right)$,

(ii) Reduction of the dielectric layer thickness $(\boldsymbol{d})$,

(iii) Enlargement of the droplet contact angle at the resting state $\left(\boldsymbol{\theta}_{\mathbf{0}}\right)$.

The first two approaches can contribute to an increased areal capacitance of the interfacial dielectric layer $(\boldsymbol{C} / \boldsymbol{A})$. Due to this enhanced capacitive property, the same amount of electric potential energy can be stored in the dielectric layer by applying a lower voltage. Therefore, the four parameters $\left(\boldsymbol{\varepsilon}_{\boldsymbol{d}}, \boldsymbol{d}, \boldsymbol{C} / \boldsymbol{A}, \boldsymbol{\theta}_{\mathbf{0}}\right)$ are relevant for a successful low-voltage EWOD electrode.

\subsubsection{Material Selection and Fabrication Methods for Dielectric Layers}

To achieve the three approaches postulated in Chapter 2.2.1.2, the key is the material, layer and surface property of the interfacial dielectric layer. The first approach, the increase of $\boldsymbol{\varepsilon}_{\boldsymbol{d}}$, can be fulfilled by using dielectric materials with high relative permittivity. The second approach, the reduction of $\boldsymbol{d}$, can be complied with thin-film deposition techniques. Moreover, the thin dielectric layer should be pin-hole free and have sufficient electrical breakdown strength. The third approach, the enlargement of $\boldsymbol{\theta}_{\mathbf{0}}$, can be achieved by increasing the surface hydrophobicity.

In the applications and earlier studies of the EWOD effect ${ }^{70-73}$, EWOD electrodes are often constructed as a dielectric bilayer or multilayer stack on an electrode substrate. The dielectric stack consists usually of an insulating material with high breakdown strength and a hydrophobic surface coating.

\section{hydrophobic coating main dielectric layer electrode substrate}

Figure 2.9. General layer structure of EWOD electrodes.

In this study, the EWOD electrode was designed based on the common layer structure (Figure 2.9): a hydrophobic coating and a main dielectric layer on an electrode substrate. The dielectric layer materials and fabrication methods were carefully selected with regard to processing approaches and optimization of low-voltage EWOD. 


\section{Anodic Tantalum Pentoxide}

A promising material for the main dielectric layer is anodic tantalum pentoxide $\left(\mathrm{Ta}_{2} \mathrm{O}_{5}\right)$. Its relative permittivity $\left(\varepsilon_{\boldsymbol{d}}=25.3-29.5\right)^{74-77}$ is much higher in comparison with the commonly used dielectrics, such as silicon oxide $\left(\varepsilon_{d}=3.9\right)^{78-79}$ and silicon nitride $\left(\varepsilon_{d}=7.5\right)^{73}$. Besides its high relative permittivity, $\mathrm{Ta}_{2} \mathrm{O}_{5}$ features high breakdown strength $(4-6.61 \mathrm{MV} / \mathrm{cm})^{76,80-81}$ and low pin-hole density ${ }^{70}, 82$. These properties have made $\mathrm{Ta}_{2} \mathrm{O}_{5}$ a favorable storage dielectric layer in microelectronics ${ }^{83}$ and in capacitance sensors ${ }^{84-85}$ over decades. Moreover, $\mathrm{Ta}_{2} \mathrm{O}_{5}$ possesses outstanding chemical stability ${ }^{82}$ and biocompatibility ${ }^{86}$ for applications in implants ${ }^{86}$ and other biomedical devices. Due to its high refractive index, $\mathrm{Ta}_{2} \mathrm{O}_{5}$ is also used as a basic material in optical waveguides ${ }^{87}$, in optical lenses ${ }^{88}$ and in antireflective films ${ }^{83}$.

$\mathrm{Ta}_{2} \mathrm{O}_{5}$ thin films can be fabricated through diverse techniques reviewed by Chaneliere et al.83: anodic or thermal oxidation of tantalum, oxide sputtering, vacuum evaporation, direct or chemical vapor deposition, atomic layer deposition, sol-gel methods, ion-assisted deposition. However, most of the techniques require expensive equipment with vacuum condition or high temperature to obtain a low-defect smooth oxide layer. In comparison, anodic oxidation (anodization) ${ }^{75,89-90}$ is a low-cost and promising fabrication method: anodic $\mathrm{Ta}_{2} \mathrm{O}_{5}$ thin layers can possess a higher film density $\left(8.01 \mathrm{~g} / \mathrm{cm}^{3}\right)^{90}$ than those obtained with other preparation methods ${ }^{83}$, higher relative permittivity (values listed above) and better layer quality (e.g. negligible pin-holes $)^{70}$ than those produced by sputtering $\left(\varepsilon_{\boldsymbol{d}}=20-23\right)^{71,91}$.

Anodization is an electrochemical process for defined oxide formation on a metal surface. The resulting layer possesses a self-healing property ${ }^{92-93}$ and a strong adherence to the metal ${ }^{94}$. For the anodization of tantalum, the following redox reaction is postulated $77,89,95$ :

$$
\begin{array}{cc}
\text { Anode reaction: } & 2 \mathrm{Ta}+5 \mathrm{H}_{2} \mathrm{O} \rightarrow \mathrm{Ta}_{2} \mathrm{O}_{5}+10 \mathrm{H}^{+}+10 \mathrm{e}^{-} \\
\text {Cathode reaction: } & 2 \mathrm{H}^{+}+2 \mathrm{e}^{-} \rightarrow \mathrm{H}_{2}, 2 \mathrm{O}_{2}+8 \mathrm{H}^{+}+8 \mathrm{e}^{-} \rightarrow 4 \mathrm{H}_{2} \mathrm{O} \\
\hline \text { Total redox reaction: } & 2 \mathrm{Ta}+\mathrm{H}_{2} \mathrm{O}+2 \mathrm{O}_{2} \rightarrow \mathrm{Ta}_{2} \mathrm{O}_{5}+\mathrm{H}_{2}
\end{array}
$$

On the anode, tantalum is oxidized to tantalum ions $\left(\mathrm{Ta}^{5+}\right)$, which react with the water molecules to form $\mathrm{Ta}_{2} \mathrm{O}_{5}$. On the cathode, hydrogen develops; oxygen is converted to water.

In this study, the anodic $\mathrm{Ta}_{2} \mathrm{O}_{5}$ was formed via potentiostatic anodization ${ }^{96}$ in citric acid on an electrode substrate of tantalum atop a silicon wafer (Chapter 3.1.2). The oxide layer thickness can be defined with process parameters, such as voltage and time.

\section{Self-assembled Silane Monolayer}

An advantageous thin layer as the hydrophobic surface coating of EWOD-electrode is an organic trichloro-silane monolayer. The silane monolayer of a few nanometers ${ }^{97}$ is significantly thinner than the commonly used spin-coated Teflon-AF film with a typical thickness from sub$\mu \mathrm{m}$ to a few $\mu \mathrm{m}^{25}$. Moreover, silane is less expensive than Teflon-AF and can form a homogeneous smooth surface ${ }^{97}$ with high-level hydrophobicity ${ }^{98}$ and low mechanical friction ${ }^{99}$. 
These features should fulfill the low-voltage EWOD approaches for a large initial contact angle and a thin coating. Furthermore, a low resistance against droplet motion should lower the voltage threshold for the EWOD-induced wetting and should ease the passive dewetting to ensure good process dynamics. Additionally, silane monolayers are good candidates for the gate dielectric in the low-voltage OTFTs (organic thin-film transistors) ${ }^{100}$ or for the functional semiconductor/dielectric interface in molecular electronic devices ${ }^{101}$.

A promising deposition method to obtain a well-arranged silane monolayer that strongly binds to the $\mathrm{Ta}_{2} \mathrm{O}_{5}$ surface, is the self-assembled monolayer (SAM) ${ }^{102}$ formation through silanization 4 . The silanization on silicon oxide and other metal oxide surfaces has been optimized over decades ${ }^{103-105}$. For the $\mathrm{Ta}_{2} \mathrm{O}_{5}$ surface, the same SAM formation principle is valid and shown in the illustration (Figure 2.10). From a microscopic view, an organic trichloro-silane molecule consists of two parts: a Si head group with three $\mathrm{Cl}$-atoms and an organic chain as rest group. Through a spontaneous chemisorption, the Si head group binds covalently onto the metal oxide surface, which ensures a superior stability of the binding to the substrate. Meanwhile, the organic chains of the silane molecules are spontaneously bound through the non-covalent van der Waal interaction. The regularly ordered and close-packed organic chains results in a hydrophobic layer. In addition, the adjacent Si head groups are bound through covalent crosslinking ${ }^{105}$, which leads to an enhanced chemical and mechanical robustness of the SAM.

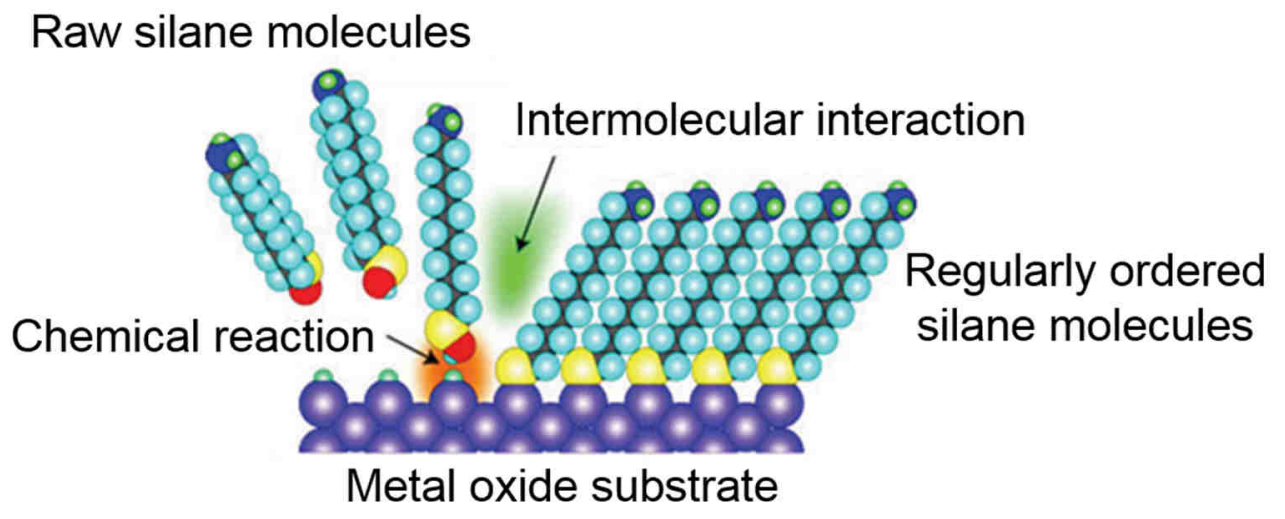

Figure 2.10. Principle of the self-assembled monolayer formation."'

In this study, the silanization on the $\mathrm{Ta}_{2} \mathrm{O}_{5}$ surface was mainly implemented in a wet chemical procedure using octadecyltrichlorosilane (ODTS) in toluene (Chapter 3.1.4).

\footnotetext{
III Own representation based on COST workshop on surface and interface 2014 in Delft.
} 


\section{Dielectric Layer Combinations}

In earlier studies, $\mathrm{Ta}_{2} \mathrm{O}_{5}$ and trichloro-silane have each been applied with diverse materials as a bilayer or multilayer dielectric integrated in advanced low-voltage EWOD electrodes. For example, $\mathrm{Ta}_{2} \mathrm{O}_{5}$ has been combined with an amorphous fluoropolymer coating, such as Teflon$\mathrm{AF}^{72}$ and Cytop ${ }^{\mathrm{TM}}$ 70-71. Li et al. showed that anodic $\mathrm{Ta}_{2} \mathrm{O}_{5}$ forms a smooth and robust layer and when coated with a thin fluoropolymer coating, the EWOD voltage could be reduced to $13 \mathrm{~V}$ 72. Moreover, anodic $\mathrm{Ta}_{2} \mathrm{O}_{5}$ was reported to have better EWOD performance under $\mathrm{AC}$ actuation $\left(\boldsymbol{U}=13 \mathrm{~V}_{\mathrm{RMS}}\right.$ ) than sputtered $\mathrm{Ta}_{2} \mathrm{O}_{5}{ }^{70}$. A bilayer stack with $\mathrm{Ta}_{2} \mathrm{O}_{5}$ was revealed to require a lower actuation threshold voltage $(\boldsymbol{U}=6 \mathrm{~V})$ than that with Parylene $\mathrm{C}$ (a poly $(\mathrm{p}$ xylylene) polymer with $\left.\varepsilon_{\mathbf{d}} \cong 3.15\right)^{71}$. Furthermore, EWOD electrodes with a hydrophobic silane integrated with other dielectric materials, such as silicon nitride was studied for electrowetting reversibility. In that study, an ODTS monolayer was shown to have slightly higher hydrophobicity and lower contact angle hysteresis than the Teflon-like fluoropolymers, which makes the droplet motion during electrowetting easier ${ }^{73}$.

In this doctoral research, an anodic $\mathrm{Ta}_{2} \mathrm{O}_{5}$ layer and a self-assembled silane monolayer are selected as the main dielectric layer and as the hydrophobic surface coating respectively; they are combined into a dielectric bilayer for the low-voltage (LV) EWOD electrode. The EWOD relevant parameters $\left(\boldsymbol{\varepsilon}_{\boldsymbol{d}}, \boldsymbol{d}, \boldsymbol{C} / \boldsymbol{A}, \boldsymbol{\theta}_{\mathbf{0}}\right)$, as mentioned in Chapter 2.2.1.1, depend on the surface, layer and material properties of both layers and can be influenced by diverse factors in the fabrication processes. To ensure the quality of the LV-EWOD electrode and to ease the comparison with other studies in the literature, it is essential to characterize each layer fabricated under diverse conditions regarding the four EWOD-relevant parameters (Chapter 4.1).

\subsubsection{Multilayer Dielectric Stack (MDS)}

In this doctoral research, the EWOD effect was studied by making use of the sessile drop model system, whereby a $\mu \mathrm{L}$-sized aqueous droplet was placed on the LV-EWOD electrode with oil as the surrounding medium. The setup is shown in the optical image (Figure 2.11 left).

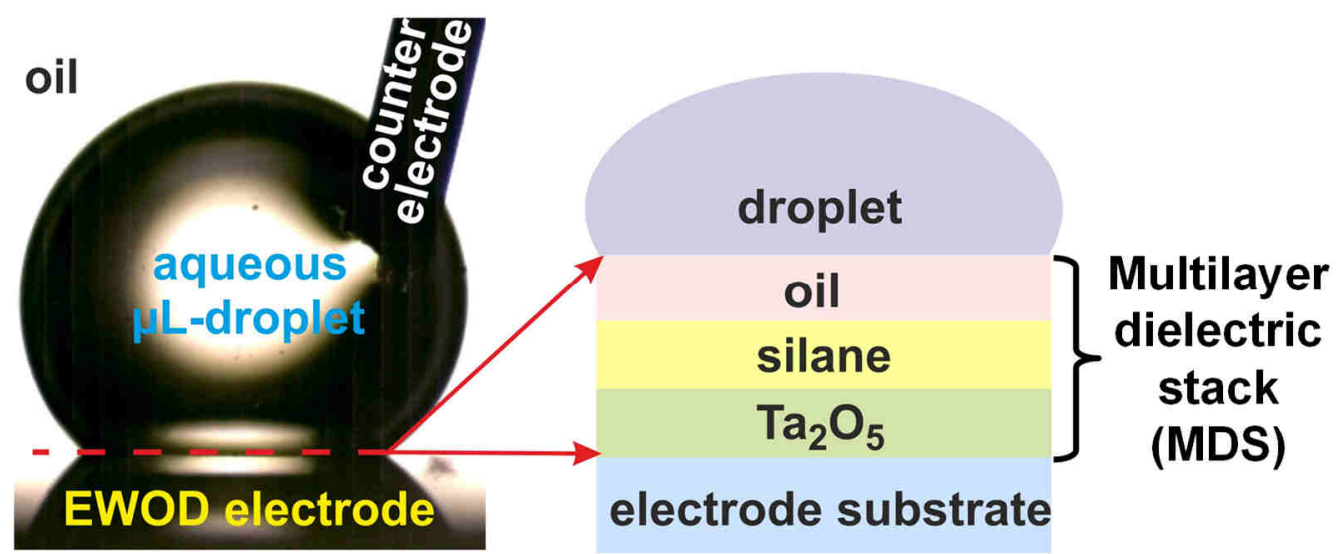

Figure 2.11. Optical image of a $3 \mu \mathrm{L}$ aqueous droplet on the EWOD electrode in oil with an inserted counter electrode and schematic abstract of the system under investigation. 
According to the Young-Lippmann equation (Eq. 2.2), the strength of the EWOD effect is directly proportional to capacitance per unit area of the dielectric material between the droplet and the electrode. This capacitance per unit area in the EWOD experiments detailed in this thesis depends directly on the components of the multilayer dielectric stack (MDS) shown in the illustration (Figure 2.11 right). The MDS consists of two solid layers in the EWOD electrode, the anodic $\mathrm{Ta}_{2} \mathrm{O}_{5}$ layer and the self-assembled silane layer, as well as one fluid oil layer entrapped between the droplet and the EWOD electrode.

This thin oil film should act as an additional dielectric layer besides the fabricated dielectric bilayer and as a lubricant to reduce the surface friction, the contact line pinning and thus ease the droplet wetting and dewetting ${ }^{35}$. The entrapment of such an oil film was observed by Staicu et al. ${ }^{36}$ in a comparable experimental setup.

\subsubsection{EWOD Effect Analysis}

\subsubsection{Equivalent Circuit Models}

To describe the system under investigation electrically, an equivalent circuit model was constructed (Figure $2.12 \mathrm{a}$ ): The aqueous droplet is represented by a resistor $\left(\boldsymbol{R}_{\text {drop }}\right)$ and a capacitor $\left(\boldsymbol{C}_{\mathbf{d r o p}}\right)$ in parallel. The MDS is described as a resistor $\left(\boldsymbol{R}_{\text {MDS }}\right)$ in parallel connection with three capacitors in series, $\boldsymbol{C}_{\mathbf{o i l}}, \boldsymbol{C}_{\text {silane }}$ and $\boldsymbol{C}_{\mathrm{Ta}_{2} \mathbf{O}_{5}}$. Concerning the measuring frequencies $(100 \mathrm{~Hz}-1 \mathrm{MHz})$ and the used salt concentrations $(6.25 \mathrm{mM}-200 \mathrm{mM} \mathrm{KCl})$ in this study, this equivalent circuit model (Figure 2.12 a) can be simplified as shown in Figure 2.12 (b).

(a)

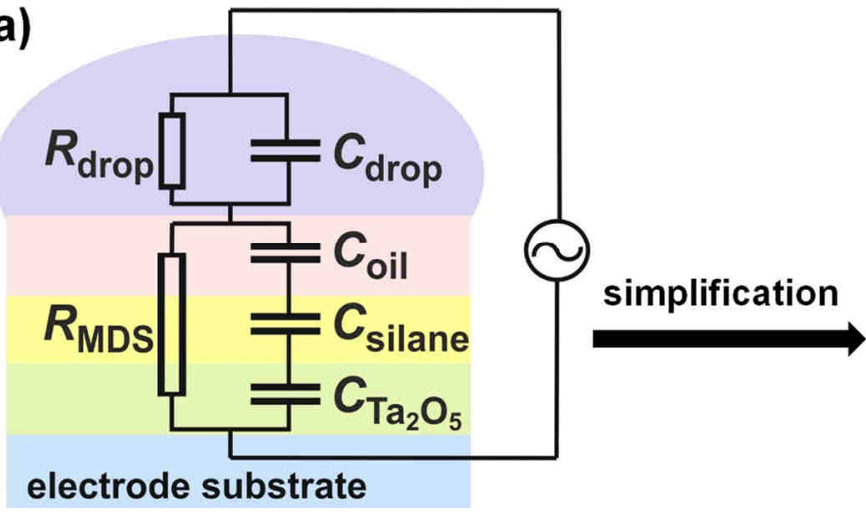

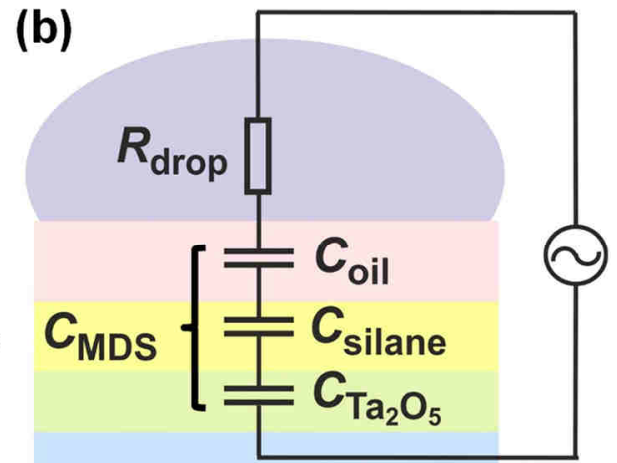

electrode substrate

Figure 2.12. (a) Equivalent circuit model for the system under investigation and (b) simplified equivalent circuit model. $R_{\mathrm{drop}}$ : droplet resistance; $C_{\mathrm{drop}}$ : droplet capacitance; $\boldsymbol{R}_{\mathrm{MDs}}$ : total resistance of the multilayer dielectric stack; $C_{\mathrm{oil}}, C_{\text {silane }}$ and $C_{\mathrm{Ta}_{2} \mathrm{O}_{5}}:$ separated capacitances of the oil film, the silane layer and the $\mathrm{Ta}_{2} \mathrm{O}_{5}$-layer respectively; $C_{\mathrm{MDs}}$ : total capacitance of the multilayer dielectric stack, composed of $C_{\text {oil }}, C_{\text {silane }}$ and $C_{\mathrm{Ta}_{2} \mathrm{O}_{5}}$ in series. 
The simplification is due to:

$$
\begin{array}{ll}
\text { (i) } & \boldsymbol{R}_{\mathrm{MDS}} \gg X_{C_{\mathrm{MDS}}} \text {, where } X_{C_{\mathrm{MDS}}}=\frac{1}{2 \pi f C_{\mathrm{MDS}}}, \\
\text { (ii) } & \boldsymbol{R}_{\text {drop }} \ll X_{C_{\text {drop }}} \text {, where } X_{C_{\text {drop }}}=\frac{1}{2 \pi f C_{\mathrm{drop}}} .
\end{array}
$$

Shapiro et al. ${ }^{106}$ suggested a comparable equivalent circuit for a system with a slightly resistive liquid drop on a dielectric resistive solid. Both their circuit and the simplified equivalent circuit in this study, describe the liquid drop as a resistor. In comparison to the simplified circuit here, the dielectric layer is modelled as a capacitor in parallel with an additional resistor. However, this resistor can be neglected under the measurement conditions in this study. Due to the low thickness of the MDS and the high relative permittivity of the $\mathrm{Ta}_{2} \mathrm{O}_{5}$-layer, the dielectric layers in this study have a very small capacitive reactance. In comparison, the total resistance of the dielectric layers is very high and appears beyond the measuring frequency range.

The EIS analysis of the EWOD effect in this study, either with the focus on the signal frequency influence or on the non-linear dynamic behavior, is based on the simplified equivalent circuit model (Figure 2.12b).

According to this model, the total capacitance of the MDS ( $\left.\boldsymbol{C}_{\text {MDS }}\right)$ is given by:

$$
\frac{1}{C_{\mathrm{MDS}}}=\frac{1}{C_{\mathrm{oil}}}+\frac{1}{C_{\text {silane }}}+\frac{1}{C_{\mathrm{Ta}_{2} \mathrm{O}_{5}}}
$$

Each layer in the MDS can be considered as a parallel plate capacitor. Thus, the capacitance per unit area $(\boldsymbol{C} / \boldsymbol{A})$ of each layer can be calculated with Eq. 2.11.

Moreover, the simplified equivalent circuit model is a series $R C$ circuit in principle, where $R$ represents the droplet and $C$ the entire MDS. Based on that, the EWOD system is comparable to a low-pass filter ${ }^{30,107}$ (Figure 2.13) in the electrical engineering. In analogy to the energy input and output through the low-pass filter, the electric potential energy stored in the MDS is the energy contributes to the EWOD effect.

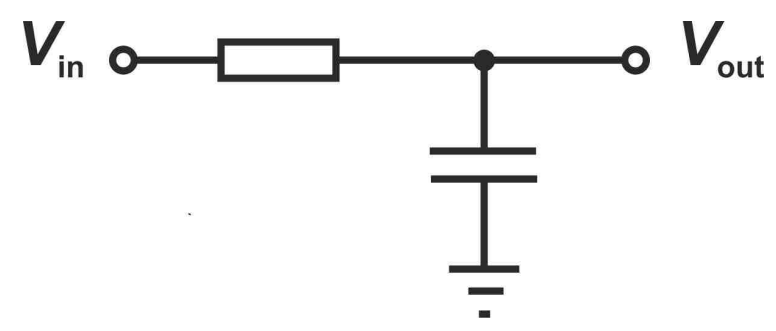

Figure 2.13. Circuit of a low-pass filter consisting of a resistor and a capacitor in series. ${ }^{\text {IV }}$ $V_{\text {in: }}$ Signal input. $V_{\text {out }}$ Signal output.

IV Own representation based on Horowitz, P.; Hill, W. The Art of Electronics. 3. ed.; Cambridge University Press: United Kingdom, 2015, p. 961. 
A low-pass filter, as its name says, passes signals with low frequencies and attenuates signals with high frequencies. The filter functionality is characterized by a cutoff-frequency, at which half of the input power is attenuated. This cutoff-frequency can be expressed identically as the critical frequency in the EIS for a series $R C$ circuit (Chapter 2.1.2.1): $f_{\mathrm{c}}=\frac{1}{2 \pi R C}$. Furthermore, the low-pass filter can also be considered as a voltage divider. At the cutoff-frequency, the voltage drops equally across the resistor and the capacitor.

\subsubsection{Energy Budget in EWOD}

In an ideal EWOD process, as described in Chapter 2.1.1, the applied electric energy at a constant voltage should be completely stored in the dielectric layer as the electric potential energy to lower the solid-liquid interfacial tension. This causes the droplet to wet the surface until a new interfacial thermodynamic equilibrium is achieved. On reducing the voltage, the droplet ideally dewets the surface reversibly and returns to its initial geometry at the resting state. The electric potential energy per unit interfacial area, as mentioned in Chapter 2.1.1.3, is given by:

$$
\frac{E_{\mathrm{el}}}{A}=\frac{1}{2} \cdot \frac{C}{A} \cdot U^{2}
$$

However, in a real EWOD system, the scenario is more complicated.

In the EWOD system of this study, an additional oil layer occupies the space between the droplet and the EWOD electrode. Under this circumstance, the stored electric potential energy can induce not only the droplet deformation, the EWOD effect, but also the deformation of the interfacial oil layer.

Besides the deformation processes, the electric energy input can be consumed in some dissipative processes: energy loss caused by the need to overcome contact line pinning due to the surface friction or through the droplet acting as a dissipative resistor and inducing Joule heating effect ${ }^{48}$.

Moreover, the impact of surface friction on EWOD can be reduced through application of an alternating voltage instead of a direct voltage as shown by Li and Mugele. ${ }^{34}$ However, by applying an alternating voltage with a high frequency, only partial electric energy input can be stored in the dielectric layer, as revealed by the low-pass filter model. The influence of frequency on the EWOD effect is reported in Chapter 4.2.

Furthermore, in either EWOD or dewetting, voltage jumps are the external disturbance for the EWOD system. By applying a single voltage jump, the EWOD- or dewetting-related deformation process should be a typical first-order relaxation response ${ }^{49}$. 
In the case of applying an asending voltage jump of the stair case stimulus (Figure 2.7) on the EWOD system, the increase of the droplet contact area $(\boldsymbol{A})$ can be expressed as:

$$
A=A_{e} \cdot\left(1-e^{-\frac{t}{\tau}}\right)
$$

with $\boldsymbol{A}_{\boldsymbol{e}}$ standing for the contact area reached at the interfacial equilibrium state and $\boldsymbol{\tau}$ for the characteristic time of the first-order relaxation process. The derivation for this equation can be found in Appendix 7.1.1. The process dynamics can be characterized with $\tau$ due to its reciprocal correlation with the process rate.

With respect to the electric potential energy, the dynamics and the non-linear responses of diverse parameters in the EWOD system have been investigated for the electrowetting and the dewetting process and shown in Chapter 4.3.

\subsubsection{Young-Lippmann Equation for Alternating Voltage Applications}

The basic Young-Lippmann equation (Eq. 2.2) is the gold standard to assess the EWOD effect at direct voltages. For alternating voltage applications, the basic Young-Lippmann equation requires adaptions. ${ }^{35}$

For the study of the influence of frequency on the EWOD effect, the Young-Lippmann equation is adapted to the measurement condition by specifying two parameters: the capacitance of the corresponding dielectric layers and the voltage inducing the EWOD effect. Moreover, the Young-Lippmann equation is extended with a correlation coefficient for comparison of EWOD performance under diverse experimental conditions and as an indicator for the energy amount converted to EWOD effect.

First, based on the EWOD system described in Chapter 2.2.1.3 and its equivalent circuit model presented in Chapter 2.2.2.1, the capacitance correlating with the EWOD effect is the MDS capacitance. Of all three dielectric layers in the MDS, the fluid oil layer can change its capacitance due to the influence of electrostatic pressure on the layer thickness ${ }^{36}$. Hence, in the adapted Young-Lippmann equation (Eq. 2.32), the entire MDS capacitance $\left(\boldsymbol{C}_{\text {MDS }}\right.$ ) is a variable parameter.

Second, the voltage involved in the Young-Lippmann equation is specified with regard to three aspects: (i) The EWOD effect is induced by a partial voltage applied to the EWOD system. It corresponds to the voltage drop across the MDS. (ii) With respect to the alternating voltage application, the effective voltage is calculated as the root mean square (RMS) value. (iii) For the EWOD electrode with an anodic $\mathrm{Ta}_{2} \mathrm{O}_{5}$ dielectric layer, merely positive voltage stimuli can be applied. This is because $\mathrm{Ta}_{2} \mathrm{O}_{5}$ is a valve metal oxide. It is a perfect insulator against the anodic current at a voltage below $50 \%$ of its anodization voltage but can be damaged by the cathodic current ${ }^{108}$. To fulfill this condition, the applied sinusoidal signal possesses a direct voltage offset that equals the voltage amplitude to yield the positive stimulus. 
Based on the equivalent circuit model (Figure $2.12 \mathrm{~b}$ ) that represents the EWOD system as a series $R C$ circuit, the specified voltage drop on the MDS, $\boldsymbol{U}_{(\mathbf{R M S}) \text { MDS }}$, can be determined by applying the voltage division principle:

$$
\boldsymbol{U}_{(\mathrm{RMS}) \mathrm{MDS}}=\frac{\boldsymbol{X}_{C_{\mathrm{MDS}}}}{|Z|} \cdot \boldsymbol{U}_{\mathrm{RMS}}
$$

Here, the capacitive reactance $\left(\boldsymbol{X}_{\boldsymbol{C}_{\text {MDS }}}\right)$ depends on the MDS capacitance $\left(\boldsymbol{C}_{\text {MDS }}\right)$ and the frequency $(\boldsymbol{f})$ :

$$
X_{C_{\mathrm{MDS}}}=\frac{1}{2 \pi f C_{\mathrm{MDS}}}
$$

The impedance modulus of the total system $(|\boldsymbol{Z}|)$ is contributed by the droplet resistance $\left(\boldsymbol{R}_{\text {drop }}\right)$ and the capacitive reactance of the MDS ( $\left.\boldsymbol{X}_{\boldsymbol{C}_{\mathrm{MDS}}}\right)$ :

$$
|Z|=\sqrt{R_{\mathrm{drop}}^{2}+X_{C_{\mathrm{MDS}}}^{2}}
$$

The RMS value of the applied positive voltage stimulus $\left(\boldsymbol{U}_{\mathrm{RMS}}\right)$ can be expressed as:

$$
U_{\mathrm{RMS}}=\sqrt{3 / 2} \cdot U_{\mathrm{pp} / 2}
$$

with $\boldsymbol{U}_{\mathbf{p p} / 2}$ standing for the amplitude, the half value of the peak-to-peak voltage. A detailed derivation of this $\boldsymbol{U}_{\mathrm{RMS}}$ expression is enclosed in Appendix 7.1.2.

Third, a dimensionless correlation-coefficient, $\boldsymbol{\beta}$, is introduced into the adapted YoungLippmann equation as below:

$$
\cos \theta_{\mathrm{u}}-\cos \theta_{0}=\beta \cdot \frac{C_{\mathrm{MDS}}}{2 \gamma_{\mathrm{lf}} A} \cdot U_{(\mathrm{RMS}) \mathrm{MDS}}^{2}
$$

$\boldsymbol{\beta}$ is considered as an assessment parameter, which enables direct comparison of electrowetting performance under diverse experimental conditions, regarding the frequency and the amplitude of the alternating voltage stimulus as well as the salt concentration.

Moreover, in the context of the energy budget in EWOD (Chapter 2.2.2.2), $\boldsymbol{\beta}$ can reflect the energy efficiency for the EWOD effect. Based on the integrated Lippmann equation (Eq. 2.7), Eq. 2.32 can be converted to the following expression:

$$
\beta=\frac{\left(\cos \theta_{\mathrm{u}}-\cos \theta_{0}\right) \cdot \gamma_{\mathrm{lf}}}{\frac{C_{\mathrm{MDS}}}{2 A} \cdot U_{(\mathrm{RMS}) \mathrm{MDS}}^{2}}=\frac{\gamma_{\mathrm{sl}(0)}-\gamma_{\mathrm{sl}(\mathrm{U})}}{\frac{C_{\mathrm{MDS}}}{2 A} \cdot U_{(\mathrm{RMS}) \mathrm{MDS}}^{2}}
$$

Here, $\boldsymbol{\beta}$ indicates the ratio of the energy output for the EWOD effect, the reduction of the solidliquid interfacial tension, to the areal electric potential energy stored in the MDS. 


\section{MATERIALS AND MethOdS}

\subsection{Fabrication of Low-Voltage (LV) EWOD Electrodes}

The LV-EWOD electrode was designed as a multilayer stack (Chapter 2.2.1.3). It was fabricated by a series of surface modifications of the planar electrode substrate (silicon wafer with a sputtered tantalum layer) as shown in the overview scheme below.

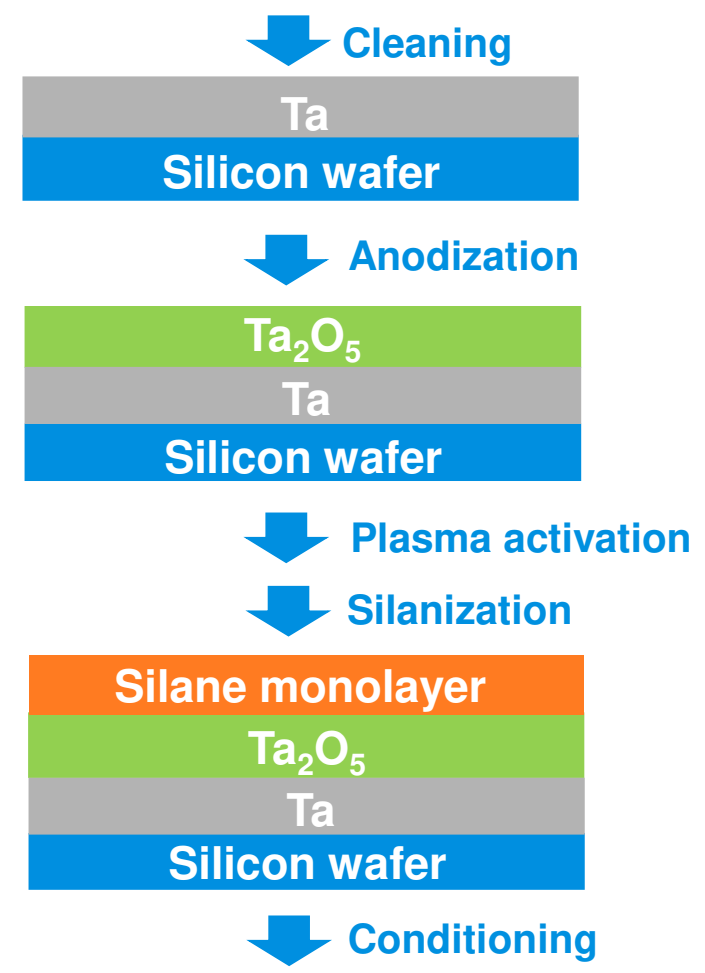

Figure 3.1. Overview scheme of the fabrication processes for the low-voltage EWOD electrode.

The fabrication process of the LV-EWOD electrode consists of 5 steps,

- cleaning (Chapter 3.1.1)

- $\quad$ anodization (Chapter 3.1.2)

- $\quad$ plasma activation (Chapter 3.1.3)

- $\quad$ silanization (Chapter 3.1.4)

- conditioning (Chapter 3.1.5).

Through these five steps, the electrode with an anodic tantalum pentoxide layer and a subsequent silane monolayer as defined dielectrics can be obtained and is conditioned for further measurement. 


\subsubsection{Electrode Substrate and Cleaning}

The electrode substrate is a planar silicon wafer without thermal oxidation that was sputter coated with a tantalum layer (typical thickness $500 \mathrm{~nm} \pm 50 \mathrm{~nm}$ ). This tantalum layer was covered with a very thin native tantalum oxide layer (typical thickness: a few $\mathrm{nm}$ ) and a photoresist layer. The electrode substrate was diced into $10 \mathrm{~mm} \times 15 \mathrm{~mm}$ specimen (supplier: IMN MacroNano ${ }^{\circledR}$, IImenau University of Technology, Germany).

A single specimen was first carefully separated from the diced substrate, which was hold by an adhesive foil from the backside (Figure 3.2).
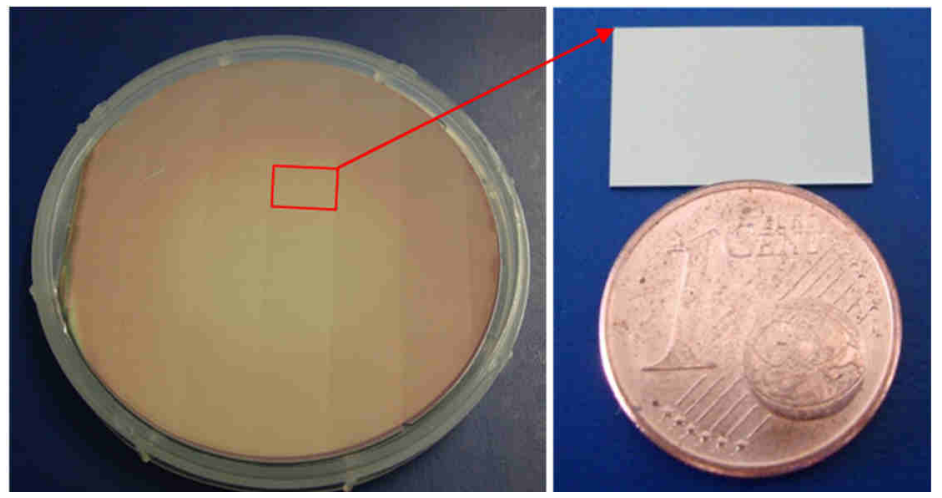

Figure 3.2. Diced substrates covered with photo resist (left). One specimen after cleaning (right). The specimen was then placed in a PTFE (Polytetrafluoroethylene) sample holder (Figure 3.3) with four vacancies for the cleaning procedures to remove the photoresist layer. This sample holder was in-house constructed and fabricated (technical drawing in Appendix 7.7: Li00).

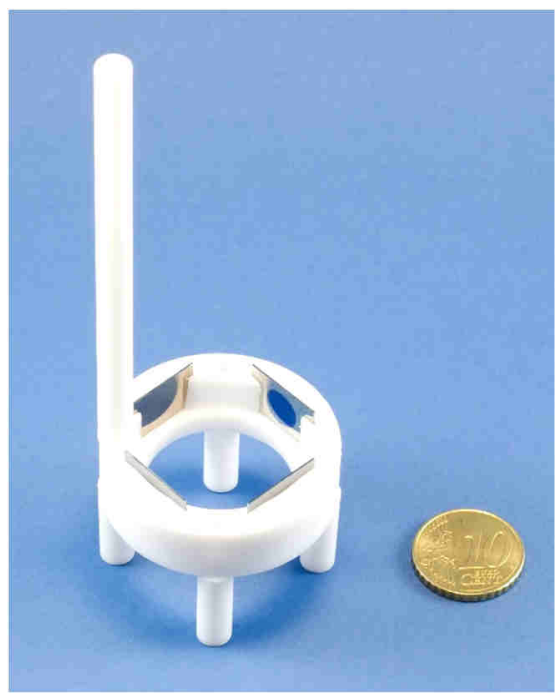

Figure 3.3. PTFE sample holder with four specimens for the cleaning procedures. 
The cleaning procedure includes three steps:

1. Immersion in an acid piranha solution, which consists of concentrated $\mathrm{H}_{2} \mathrm{SO}_{4}$ (Carl Roth $\mathrm{GmbH}$, Germany) and $30 \% \mathrm{H}_{2} \mathrm{O}_{2}$ (Carl Roth $\mathrm{GmbH}$, Germany) in a ratio of 3:1, at room temperature under stirring for $15 \mathrm{~min}$.

2. Heating in a base piranha solution, which consists of $25 \% \mathrm{NH}_{4} \mathrm{OH}$ (Carl Roth $\mathrm{GmbH}$, Germany), $30 \% \mathrm{H}_{2} \mathrm{O}_{2}$ and deionized water in a ratio of $1: 1: 4$, at $70^{\circ} \mathrm{C}$ under stirring for $15 \mathrm{~min}$.

3. Rinsing with deionized water and keep the specimen in the deionized water before usage.

\subsubsection{Anodization}

The anodization, as shown in Figure 3.4, is conducted under the potentiostatic condition in an electrochemical cell. The anode is here the working electrode (WE). On the anode surface, the electrochemical oxidation of tantalum to tantalum pentoxide occurs and the generated electrons enter the electrical circuit. The cathode is the counter electrode (CE), from which the electrons are released for the electrochemical reduction. Both electrodes are connected to a power source supplying a constant DC voltage. The current flowing through the circuit is measured by an ammeter. In this work, an electrometer is used as the power source and the ammeter.

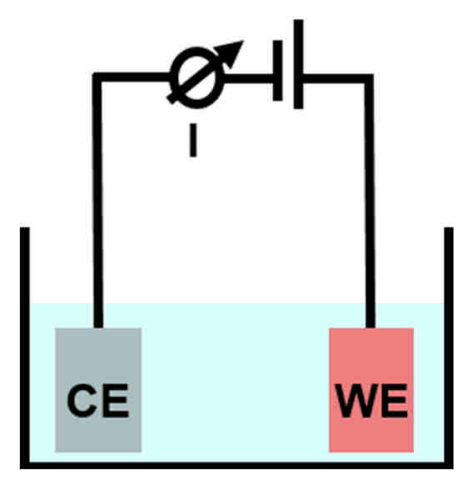

Figure 3.4. Scheme of an electrochemical cell configured with two electrodes for potentiostatic anodization and an ammeter for current measurement. CE: counter electrode, WE: working electrode.

\section{Setup}

The setup for the anodization consists of the following six elements: an anodization cell containing the specimen, a counter electrode, a solution bath, a Faraday cage, an electrometer and a control and recording software.

One essential accessory for the anodization was an anodization cell (Figure 3.4), in which the specimen was mounted for anodization in a solution bath. 
This anodization cell provides two functionalities: (i) It strictly separates the specimen surface in two regions: A round opening in the middle ensures a defined section area $\left(0.42 \mathrm{~mm}^{2}\right)$ for the anodization; meanwhile, the rest of the specimen is leak-proof to avoid short current. (ii) In the leak-proof region, the specimen inside is electrically connected with a spring pin wired to a power source for the anodization. The anodization cell was in-house constructed and fabricated (technical drawing in Appendix 7.7: Li 01).
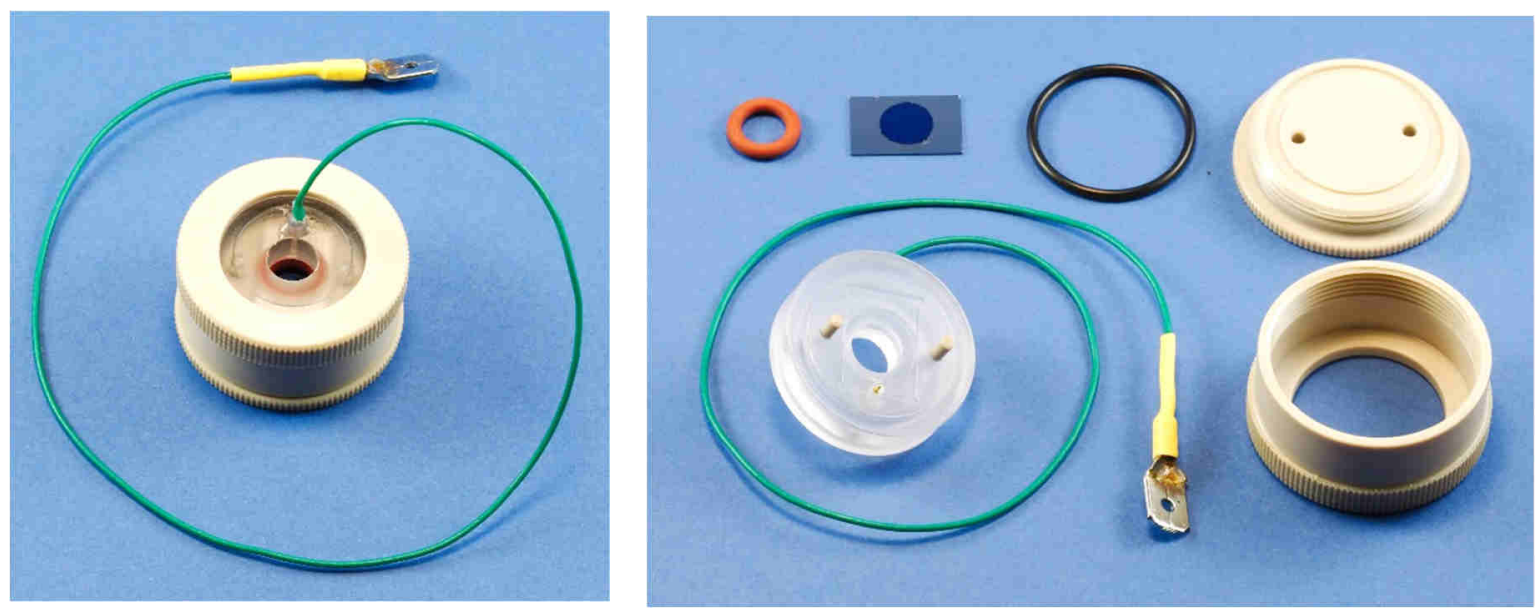

Figure 3.5. Anodization cell (left) facilitates a defined round area of the tantalum as the working electrode (WE). Separated anodization cell (right): two seal rings, housing bottom, top housing nut and middle part with a central aperture and a wired spring pin.

An electrometer (Keithley 6517, Tektronix Inc., USA) was used for DC-voltage application. A graphite electrode (Phywe Systeme $\mathrm{GmbH} \&$ Co. KG, Germany) was utilized as the counter electrode. An in-house fabricated Faraday cage was used for shielding during the anodization process.

As the measuring software for the anodization, a LabView program ${ }^{\vee}$ was developed in this work to fulfill the following demands: (i) setting the duration for the anodization, (ii) automatic countdown of the anodization period (iii) tracing the real-time current flow and recording the data every 0.45 seconds in a txt. file during the anodization process.

\section{Procedure}

Directly before the anodization, the specimen was rinsed with deionized water, dried with nitrogen flow and mounted in the anodization cell. The anodization cell was completely immersed in a solution bath of degassed $0.1 \mathrm{M}$ aqueous citric acid. The specimen was connected through the anodization cell as the working electrode to the electrometer. Opposite to the opening of the anodization cell, the graphite electrode was inserted in the solution and contacted to the electrometer as the counter electrode.

\footnotetext{
$\checkmark$ The development of the LabView program was mostly supported by Robert Römer (Bioprocessing Techniques Department) and by Dr. Brian Cahill (Junior Researcher Department) at IBA.
} 
This electrochemical cell was located in the Faraday cage. Both electrode surfaces were controlled for assurance of no attached gas bubbles.

The electrometer was set to apply a constant positive DC voltage $(20,30,40$ or $50 \mathrm{~V})$ on the tantalum specimen against the counter electrode. An anodization duration of 90 min was set in the LabView program. The real-time current value was traced and shown in the LabView program window. A representative development of the current density over time is plotted for the anodization process at $30 \mathrm{~V}$ in Figure 3.6.

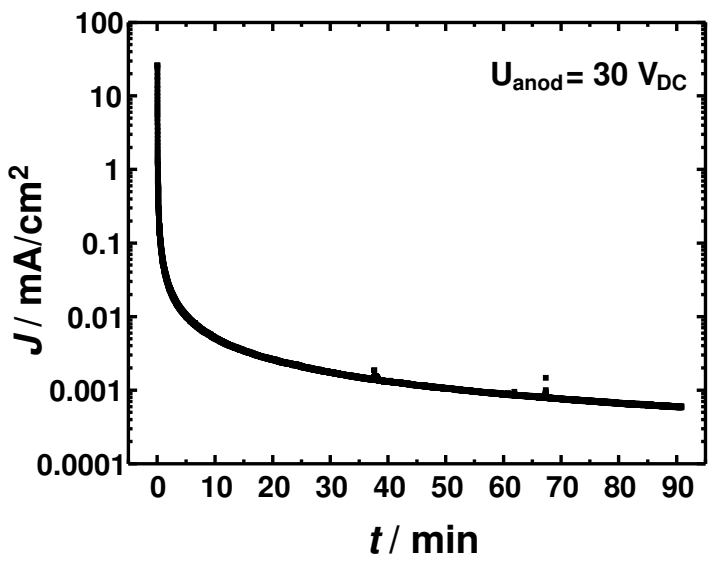

Figure 3.6. Logarithmic plot of current density over time for a representative anodization process of tantalum at $30 \mathrm{~V}$ in $0.1 \mathrm{M}$ citric acid for $90 \mathrm{~min}$.

After the anodization, the specimen was analyzed by EIS using the same anodization cell and $0.1 \mathrm{M}$ citric acid. Afterwards, the specimen was demounted from the anodization cell, cleaned with the acid piranha solution (Chapter 3.1.1, Cleaning step 1), rinsed with deionized water and dried with nitrogen flow for the further fabrication.

\subsubsection{Plasma Activation}

A plasma is an ionized gas, the so-called "fourth state of matter", and was firstly studied by Irving Langmuir ${ }^{109-110}$. Plasma is typically formed by applying an electric current or radio waves to a gas at low pressure. In this process, the gas molecules are heated, collide with each other, generate free electrons and become ionized. The free electrons collide with the gas molecules and generate more electrons. This cascade process continues until an equilibrium state, at which a certain degree of ionization is reached. ${ }^{111}$ Plasma has diverse applications, such as plasma medicine ${ }^{112}$, light generation with extreme brightness ${ }^{113}$, plasma etching and deposition in the semiconductor production ${ }^{14-115}$. Here, an oxygen plasma was applied to oxidize and to remove the residual organic contamination on the $\mathrm{Ta}_{2} \mathrm{O}_{5}$ surface as well as to activate the surface for the subsequent silane coating. 


\section{Procedure}

Immediately before the silanization step, the dried specimen was placed in the middle of the plasma cleaner chamber (Plasma Cleaner, Harrick Inc., USA). The chamber pressure was firstly reduced to 26.7 $\mathrm{Pa}$ and subsequently increased through an oxygen influx to 66.6 Pa. At this condition, an oxygen plasma was generated. The specimen was treated with the oxygen plasma for 1 minute.

\subsubsection{Silanization}

Octadecyltrichlorosilane (ODTS) was selected as the appropriate coating material for fabricating the hydrophobic surface of the LV-EWOD electrode. It consists of a silane functional group and a long alkane chain as revealed by its chemical structure (Figure 3.7).<smiles>CC(C)(C)CC(C)(C)C[Si](Cl)(Cl)Cl</smiles>

Figure 3.7. Chemical structure of octadecyltrichlorosilane (ODTS).

The silanization in this study was a wet-chemical coating process with an ODTS (Abcr, Germany) solution in toluene (Sigma-Aldrich Chemie, Germany). Since ODTS is sensitive to moisture, its contamination with air at the normal ambient humidity can induce unwanted agglomeration of ODTS. To assure a dry ambiance for the silane solution preparation, a glove bag (Aldrich® AtmosBag, Sigma-Aldrich, Germany) was set up, equipped with a hygrometer and associated with a vacuum plant and with a dry argon gas supply. The toluene was dried over night with sodium sulphate (Carl Roth, Germany). The consumable items, such as pipette tips and glass tubes, were also dried before usage.

\section{Procedure}

First, the glove bag with all items for the silane solution preparation inside was alternatively evacuated and filled with dry argon gas.

Second, a $0.5 \mathrm{wt} \%$ ODTS solution was prepared in the dried toluene under argon atmosphere in the glove bag.

Third, the ODTS coating was performed according to the literature ${ }^{116}$ with modification. After the plasma activation (Chapter 3.1.3), the specimen was immediately immersed in the prepared ODTS solution under argon atmosphere for 3 hours and kept in the dark.

Fourth, the specimen was thoroughly and successively rinsed with chloroform, acetone, deionized water and methanol, blown off with nitrogen gas and dried for 12 hours at $80^{\circ} \mathrm{C}$ in a heating cabinet. 


\subsubsection{Conditioning}

The fabricated EWOD electrode was conditioned in tetradecane (Sigma-Aldrich Chemie, Germany) before its usage in all experiments performed in oil. As transparent liquid oil, tetradecane is a long-chain alkane insoluble in water $\left(3.3 \cdot 10^{-4} \mathrm{mg} / \mathrm{L}\right)^{117}$. For the conditioning, the entire specimen was immersed in tetradecane and kept in the dark at least for one day. 


\subsection{Characterization of Dielectric Layers}

This section describes the characterization of diverse surface and layer properties of the dielectric materials consisting in the LV-EWOD electrode using commercial equipment. The characterization refers to the electrowetting-relevant parameters declared in Chapter 4.1.1. It aims to select the appropriate fabrication conditions and to validate the quality of the dielectric materials for the LV-EWOD electrode. The characterization covers the following analysis spectrum:

- Capacitance determination with electrical impedance spectroscopy (EIS) in a bulk solution and in a drop of solution (Chapter 3.2.1)

- Characterization of capacitive behavior with the cyclic voltammetry (CV) in a drop of solution (Chapter 3.2.2)

- Layer thickness measurement with the ellipsometry (Chapter 3.2.3)

- Surface topology and roughness investigation with the atomic force microscopy (AFM, Chapter 3.2.4)

- Surface chemistry analysis by means of the contact angle measurement (Chapter 3.2.5) and determination of the contact angle hysteresis (Chapter 3.2.6)

For the measurements in this section, the following aqueous solutions were prepared with the deionized water: (i) $0.1 \mathrm{M}$ citric acid solution after degassing for $30 \mathrm{~min}$ and (ii) $200 \mathrm{mM} \mathrm{KCl}$ in $0.1 \mathrm{mM} \mathrm{HCl}$ solution.

\subsubsection{Electrical Impedance Spectroscopy: Capacitance}

Electrical impedance spectroscopy (EIS) is a non-invasive analytic method that finds use in versatile applications (Chapter 2.3). In this study, potentio electrochemical impedance spectroscopy (PEIS) was applied as the measurement technique. To determine the capacitance of the dielectric coatings, the impedance spectra were analyzed with an equivalent circuit model, which electrically represents the system under investigation. The areal value of the capacitance can be obtained with reference to the involved electrode area.

In this chapter, the capacitance per unit area was determined with a commercial potentiostat associated in two measurement setups for different dielectric layers:

(i) Bulk solution setup for the native tantalum oxide layer and the anodic $\mathrm{Ta}_{2} \mathrm{O}_{5}$-layers $\left(\boldsymbol{U}_{\text {anod }}=20-50 \mathrm{~V}\right)$

(ii) Drop solution setup for the $\mathrm{Ta}_{2} \mathrm{O}_{5}$-ODTS-layer 


\subsubsection{EIS in a Bulk Solution}

To determine the capacitance per unit area $(\mathrm{C} / \mathrm{A})$ of the native tantalum oxide layer and the anodic $\mathrm{Ta}_{2} \mathrm{O}_{5}$-layers $\left(\boldsymbol{U}_{\text {anod }}=20-50 \mathrm{~V}\right)$, the PEIS measurements were performed in a bulk solution.

\section{Setup}

The setup was an electrochemical cell with a potentiostat and three electrodes in a bulk solution. As shown in Figure 3.8, the potentiostat serves as the power source, generates the voltage signal and traces the current feedback. The defined voltage signal was applied between the working electrode (WE) and the reference electrode (RE). The current flow was measured between the working electrode and the counter electrode (CE). Here, single sinusoidal signals were used for measurements in a defined frequency range (Table 3.1).

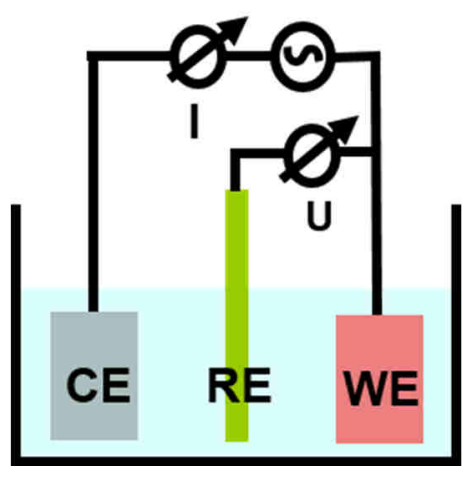

Figure 3.8. Scheme of an electrochemical cell configured with three electrodes in a bulk solution and a potentiostat for the electrical impedance spectroscopy. CE: counter electrode, RE: reference electrode, WE: working electrode.

\section{Procedure}

First, the working electrode was prepared: In case of the native tantalum oxide layer, an electrode substrate was cleaned (Chapter 3.1.1) and mounted into the anodization cell (Figure 3.5). In case of an anodic $\mathrm{Ta}_{2} \mathrm{O}_{5}$-layer, an electrode substrate was cleaned and anodized (Chapter 3.1.2). The anodized electrode substrate was kept inside the anodization cell.

Second, the electrode substrate was connected through the anodization cell as working electrode to a potentiostat (SP-300, Bio-Logic Science Instruments SAS, France) against a graphite counter electrode (44513-00, Phywe System, Germany) and a silver / silver chloride reference electrode (DRIREF-2, Sensortechnik Meinsberg GmbH, Germany). The three electrodes were inserted in the $0.1 \mathrm{M}$ citric acid solution. 
The PEIS measurement was conducted under the instruction ${ }^{118-119}$ for using the software, EClab V10.44 (Bio-Logic Science Instruments SAS, France), with the parameters listed in Table 3.1.

Table 3.1. Parameters for the PEIS measurements of the $\mathrm{Ta}_{2} \mathrm{O}_{5}$ layer with EC-lab.

\begin{tabular}{cc}
\hline parameter & setting \\
mode & single sine \\
frequency range & $1 \mathrm{mHz}-10 \mathrm{kHz}$ \\
number of data points & 7 per decade \\
voltage offset & $0.01 \cdot \boldsymbol{U}_{\text {anod }}$ (anodic oxide layer); $0.05 \mathrm{~V}$ (native oxide layer) \\
sinus amplitude & $0.01 \cdot \boldsymbol{U}_{\text {anod }}$ (anodic oxide layer); $0.05 \mathrm{~V}$ (native oxide layer) \\
\hline
\end{tabular}

Third, the electrode substrate was demounted from the anodization cell after the PEIS measurement. It was rinsed with the deionized water and dried with the nitrogen flow.

\section{Data Processing}

The recorded impedimetric data were processed and analyzed with the same software, EClab V10.44, according to the user manual ${ }^{119}$. The impedance spectra were plotted as Bode plots with the impedance modulus and the phase shift against the frequency (Figure $3.9 \mathrm{a}$ ).

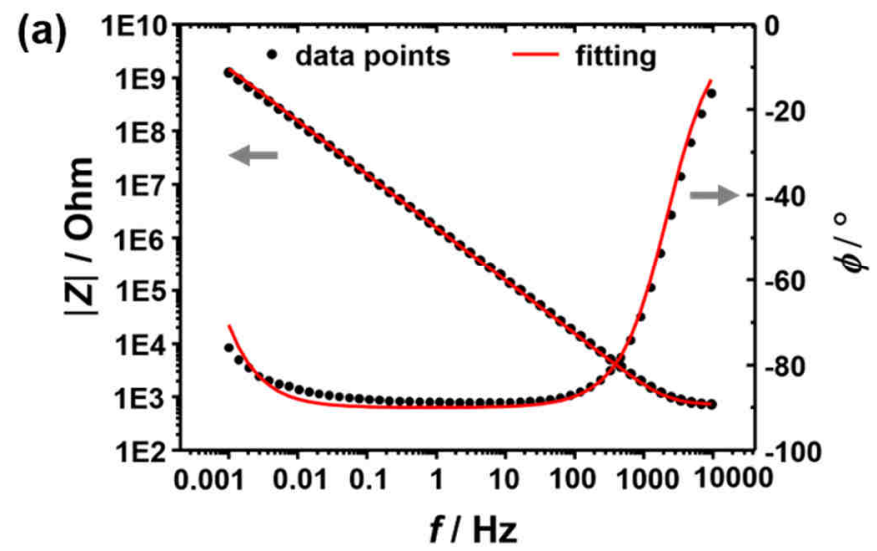

(b)

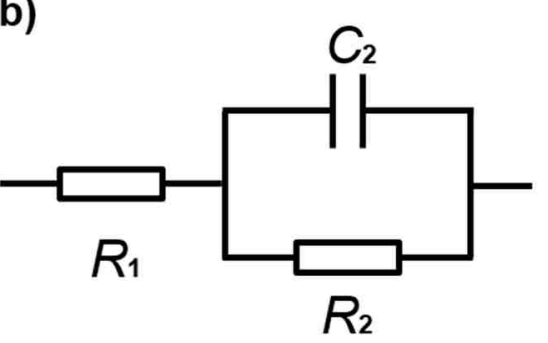

Figure 3.9. (a) Bode plot (black dots) of impedance modulus (left axis) and phase shift (right axis) against frequency for $\mathrm{a}_{2} \mathrm{O}_{5}$ layer anodized at $50 \mathrm{~V}$ measured with PEIS. The fitted curves (red) based on (b) Equivalent circuit model for the tantalum oxide layer and the measurement solution. $R_{1}$ : Resistance of the measurement solution, $C_{2}, R_{2}$ : Capacitance and resistance of tantalum oxide layer.

The data were then fitted with the equivalent circuit model illustrated in Figure 3.9 (b) with the same software for the measurements. 
The fitting was performed with the parameters listed in Table 3.2 to determine the value of $C_{2}$. It indicates the capacitance of the $\mathrm{Ta}_{2} \mathrm{O}_{5}$ layer. The capacitance per unit area of the $\mathrm{Ta}_{2} \mathrm{O}_{5}$ layer was calculated with reference to the electrode area $\left(0.42 \mathrm{~mm}^{2}\right)$. This was specified by the central aperture of the anodization cell.

Table 3.2. Parameters for the data fit with EC-lab.

\begin{tabular}{cc}
\hline fit parameter & setting \\
analysis tool & Z-Fit \\
equivalent circuit & $R_{1} /\left(R_{2}+C_{2}\right)$ \\
fit method & Randomize + Simplex \\
weight & $|Z|$ \\
iterations (Randomize) & 5000 \\
iterations (Simplex) & 10000 \\
\hline
\end{tabular}

\subsubsection{EIS on a Droplet of Solution}

To determine the capacitance per unit area of the $\mathrm{Ta}_{2} \mathrm{O}_{5}$-ODTS-bilayer, the PEIS measurement was conducted with two electrodes connected with a droplet of solution. These measurements also served as the calibration for the in-house developed EIS setup involved in the LV-EWODEIS system (Chapter 3.3.1-3.3.2).

\section{Setup}

The setup consisted of the commercial potentiostat (SP-300, Bio-Logic Science Instruments SAS, France), an in-house-fabricated measurement cell (Chapter 3.3.1) and an imaging system (Chapter 3.3.1).

In comparison with the PEIS measurements in a bulk solution with three electrodes (Figure 3.8), this electrochemical cell solely consisted of the potentiostat, a working electrode (WE) and a counter electrode (CE). Instead of a bulk solution, a droplet of solution connected the two electrodes electrically. The working electrode was the fabricated LV-EWOD electrode with a $\mathrm{Ta}_{2} \mathrm{O}_{5}$ layer anodized at $30 \mathrm{~V}$ as the initial layer and an ODTS top layer. The counter electrode was a miniaturized platinum electrode with a wire diameter of $0.23 \mathrm{~mm}$. This electrochemical cell was set up in a measurement cell (Chapter 3.3.1).

The potentiostat worked as the power source, the signal generator and the signal recorder. The imaging system was applied to determine the contact area of the working electrode to the drop (Chapter 3.3.1). 


\section{Procedure}

A fabricated LV-EWOD electrode without the oil conditioning was fixed in the in-house fabricated electrode-holder (Figure 3.13, Chapter 3.3.1). Through the holder, the electrode was connected through a spring pin as the working electrode to the potentiostat. The electrode holder was placed on a sample stage (Table 3.10, Chapter 3.3.1). Through a holding bar (Figure 3.13, Chapter 3.3.1) on the lifting stage, the platinum electrode was fixed right above the LV-EWOD electrode within the area of the anodic $\mathrm{Ta}_{2} \mathrm{O}_{5}$. The platinum electrode was connected as the counter electrode to the potentiostat.

Immediately before the PEIS measurement, a $3 \mu \mathrm{L}$ droplet of $200 \mathrm{mM} \mathrm{KCl}$ was positioned between the platinum wire and the LV-EWOD electrode surface. The PEIS measurement was conducted according to the user instruction ${ }^{118-119}$ with the following setting:

Table 3.3. Parameters for the PEIS measurements of the ODTS-Ta2 $\mathrm{O}_{5}$-bilayer with EC-lab.

\begin{tabular}{cc}
\hline measurement parameter & setting \\
mode & single sine \\
frequency range & $100 \mathrm{~Hz}-10 \mathrm{kHz}$ \\
number of data points & 5 per decade \\
voltage offset & $0.5 \mathrm{~V}$ \\
sine amplitude & $0.5 \mathrm{~V}$ \\
\hline
\end{tabular}

Since the applied voltage was below the threshold for the EWOD effect, the droplet geometry maintained its static resting state. Shortly after the PEIS measurement was started, the imaging system was operated with a software written in Python (Chapter 3.3.1). The optical measurement was focused on the interfacial region of the droplet on the LV-EWOD-electrode. The diameter of the droplet-electrode contact area was determined with the profile images recorded in the optical measurement (Chapter 3.3.1). Both the PEIS measurement and the imaging acquisition took a few seconds.

\section{Data Processing}

The data fitting was performed according to the user manual ${ }^{119}$. A simplified equivalent circuit model with one resistor and one capacitor in a series connection (Figure 3.10) was used in the Z-Fit, the EC-lab analysis tool.

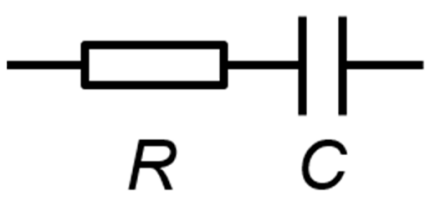

Figure 3.10. Equivalent circuit model for the $\mathrm{Ta}_{2} \mathrm{O}_{5}-\mathrm{ODTS}$-bilayer and the drop of solution. $R$ : Resistance of the measurement solution, $C$ : Capacitance of the bilayer. 
In this equivalent circuit model, the resistor represents the resistance of the measuring solution and the capacitor stands for the total capacitance of the $\mathrm{Ta}_{2} \mathrm{O}_{5}$-ODTS-bilayer. For the frequency measuring range (Table 3.3), the resistance of the dielectric bilayers does not appear in the spectrum due to its very high value. Therefore, the equivalent circuit can be simplified in comparison to that in Figure 3.9 (b).

The other parameters for the data fitting were identical to those listed in the Table 3.2. Through the data fitting, $\boldsymbol{C}$ was determined. The contact area value $(\boldsymbol{A})$ was calculated with the measured contact diameter $\left(\boldsymbol{d}_{\mathbf{c}}\right)$ according to the correlation $\boldsymbol{A}=\mathbf{1} / \mathbf{4} \cdot \boldsymbol{\pi} \cdot \boldsymbol{d}_{\mathbf{c}}{ }^{2}$. The capacitance per unit area of the $\mathrm{Ta}_{2} \mathrm{O}_{5}$-ODTS-bilayer was obtained with reference to the value of the contact area between the droplet and the LV-EWOD-electrode.

\subsubsection{Cyclic Voltammetry: Capacitive Behavior}

Cyclic voltammetry (CV) ${ }^{120-121}$ is commonly used in electrochemical analysis for studying electrode coatings and electrochemical processes on the electrodes. The CV measurement was performed in this study with a droplet of solution in an immiscible oil surrounding to characterize the capacitive behavior of the MDS comprising the solid $\mathrm{Ta}_{2} \mathrm{O}_{5}$-ODTS-bilayer and a fluid oil layer as dielectrics.

\section{Setup}

The measurement setup was nearly identical to that for the PEIS measurement on a drop of solution (Chapter 3.2.1.2). It consisted of the commercial potentiostat (SP-300, Bio-Logic Science Instruments SAS, France), the imaging system for the EWOD-EIS system (Chapter 3.3.1) and the measurement cell (Chapter 3.3.1).

The CV measurements was performed on a droplet in oil contained in the measurement cell. The electrochemical cell was comparable to that for the PEIS measurements with a bulk solution. Instead of a bulk solution, a drop of solution in oil connected the three electrodes (Figure 3.11).

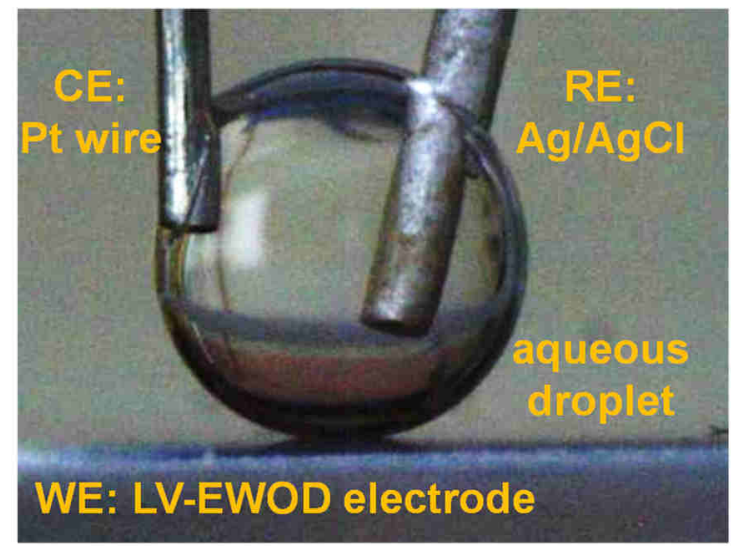

Figure 3.11. Optical image of the electrochemical cell for cyclic voltammetry (CV) measurements in an aqueous droplet connecting three electrodes in oil. WE: LV-EWOD electrode as working electrode, $\mathrm{CE}$ : platinum wire as counter electrode, $\mathrm{RE}$ : sintered $\mathrm{Ag} / \mathrm{AgCl}$ wire as reference electrode. 
The counter electrode (CE) and the reference electrode (RE) were inserted in the droplet resting on the working electrode (WE). In this study, the working electrode was a LV-EWOD electrode with a $\mathrm{Ta}_{2} \mathrm{O}_{5}$ layer $\left(\boldsymbol{U}_{\text {anod }}=30 \mathrm{~V}\right)$ and an ODTS top layer in association with a thin oil layer beneath the drop. The counter electrode was the miniaturized platinum electrode with a platinum wire and the reference electrode was a sintered $\mathrm{Ag} / \mathrm{AgCl}$ wire.

The potentiostat and the imaging system were used to fulfill the same tasks as described in Chapter 3.2.1.2.

\section{Procedure}

Comparable to the PEIS measurement on a droplet, a LV-EWOD electrode was fixed in the electrode holder (Figure 3.13, Chapter 3.3.1). The electrode holder was positioned in tetradecane (oil) contained by a glass cuvette (Figure 3.13, Chapter 3.3.1.) and connected to the potentiostat as the working electrode. A $10 \mu \mathrm{L}$ droplet of $200 \mathrm{mM} \mathrm{KCl}$ solution was placed in tetradecane on the LV-EWOD electrode within the region of the anodic $\mathrm{Ta}_{2} \mathrm{O}_{5}$ layer. The platinum electrode and the sintered $\mathrm{Ag} / \mathrm{AgCl}$ wire were inserted into the drop (Figure 3.11) and connected to the potentiostat as the counter electrode and as the reference electrode respectively.

The CV measurements were performed according to the user manuals ${ }^{118-119}$ for the software, EC-lab V10.44 (Bio-Logic Science Instruments SAS, France). The applied electrical signal processed a triangle waveform with the parameter settings listed in Table 3.4.

Table 3.4. Parameters for the CV measurements with EC-lab.

\begin{tabular}{cc}
\hline CV-parameter & setting \\
\hline starting potential & $0.1 \mathrm{~V}$ vs. RE \\
scan rate & $32 \mathrm{mV} / \mathrm{s}$ (oil) \\
maximal potential & $0.4 \mathrm{~V}$ vs. RE \\
minimal potential & $0.1 \mathrm{~V}$ vs. RE \\
cycle numbers & 3 \\
\hline
\end{tabular}

Since the applied maximal potential $(0.4 \mathrm{~V}$ vs. RE) was below the electrowetting excitation threshold, the droplet presented no electrowetting behavior and remained in a static resting state during the entire CV measurement. Analog to the imaging measurement for PEIS on a drop of solution (Chapter 3.2.1.2), the diameter of the contact area between the droplet and the LV-EWOD electrode was measured with the imaging system operated with the Python script (Chapter 3.3.1). 


\section{Data Processing}

The recorded data were processed with the software, EC-lab V10.44, according to the user manual ${ }^{119}$. A baseline correction was performed to the current data with the program Origin (OriginPro 8.5, OriginLab Corporation, United States). The cyclic voltammogram was obtained by plotting the current response against the applied voltage between the working electrode and the reference electrode.

\subsubsection{Ellipsometry: Layer Thickness}

Ellipsometry ${ }^{122-123}$ is a non-contact and non-destructive spectroscopic analysis method for thin film characterization concerning the layer thickness and the optical properties (reflection index, extinction coefficient). In this study, imaging ellipsometry was applied to determine the thickness of the anodic $\mathrm{Ta}_{2} \mathrm{O}_{5}$ layers $\left(\boldsymbol{U}_{\text {anod }}=20,30,40\right.$ and $\left.50 \mathrm{~V}\right)$ and that of the ODTS coating.

\section{Principle}

The general principle ${ }^{123}$ of ellipsometry is based on the change of polarization states of a polarized light beam reflected on a surface. The change or maintenance of the polarization state depends on the optical properties (refraction index, extinction coefficient) of the sample material as well as on the layer thickness. The polarization state is described by the amplitude and the phase shift of the $p$ component (parallel to the incident plane) and $s$ component (perpendicular to the incident plane) of the polarized light. As shown in the fundamental equation of ellipsometry ${ }^{124}$ (Eq. 3.1), the polarization state change is experimentally determined with two ellipsometric parameters, Delta $(\boldsymbol{\Delta})$ and Psi $(\boldsymbol{\psi})$ :

$$
\tan \psi e^{i \Delta}=\frac{R^{P}}{R^{S}}
$$

Delta relates to the phase shift difference between the $p$ and the $s$ component. Psi corresponds to the amplitude reduction, which is the reflection coefficient $(\boldsymbol{R})$ ratio between the $p$ and the $s$ component.

\section{Procedure}

To determine the different thicknesses of the anodic $\mathrm{Ta}_{2} \mathrm{O}_{5}$ layers, specimens under investigation were the electrode substrates with the $\mathrm{Ta}_{2} \mathrm{O}_{5}$ layers fabricated through anodization at 20, 30, 40 and $50 \mathrm{~V}$ respectively (Chapter 3.1.2). Shortly before the ellipsometry measurement, the specimen was cleaned again with the acid piranha solution (Chapter 3.1.1, cleaning step 1). The ellipsometry measurements were conducted according to the operating instructions $^{125}$ using a spectroscopic imaging ellipsometer (EP3). The measurement parameters were listed in Table 3.5. 
To obtain the ODTS layer thickness, a prerequisite was the precisely known thickness of the $\mathrm{Ta}_{2} \mathrm{O}_{5}$ initial layer. First, an electrode substrate with an anodic $\mathrm{Ta}_{2} \mathrm{O}_{5}$ initial layer $\left(\boldsymbol{U}_{\text {anod }}=30 \mathrm{~V}\right)$ was measured with the ellipsometer (EP3) to determine the $\mathrm{Ta}_{2} \mathrm{O}_{5}$ layer thickness. Second, this electrode substrate was activated with the oxygen plasma (Chapter 3.1.3) and coated with an ODTS layer (Chapter 3.1.4). After the LV-EWOD electrode was fabricated, the ellipsometry measurement was performed with the parameter settings listed in Table 3.5.

Table 3.5. Parameters for the ellipsometric measurements of $\mathrm{Ta}_{2} \mathrm{O}_{5}$ layers and ODTS coating ${ }^{\mathrm{v}}$.

measurement parameter

wavelength $(\boldsymbol{\lambda})$

microscope objective

angle of incidence (AOI)

region of interest $(\mathrm{ROI})$

measurement averaging anodic $\mathrm{Ta}_{2} \mathrm{O}_{5}$ layers

$658 \mathrm{~nm}, 532 \mathrm{~nm}$

20x magnification

$70^{\circ}$

3 ROls

4-zone

\section{ODTS coating}

$532 \mathrm{~nm}$

20x magnification

$40^{\circ}$

3 ROls

\section{Data Processing}

The data processing was performed according to the user manual ${ }^{126}$ for the analyzing software (EP4 Model 1.2.0). With the software, a sample layer stack was set up as model. Based on the fabrication process of the LV-EWOD electrode (Figure 3.1), the model for the substrate with an anodic $\mathrm{Ta}_{2} \mathrm{O}_{5}$ layer consists from bottom up of $\mathrm{Ta}, \mathrm{Ta}_{2} \mathrm{O}_{5}$ and air. For the LV-EWOD electrode, ODTS was added between $\mathrm{Ta}_{2} \mathrm{O}_{5}$ and air in the model. The ellipsometry data were fitted according to the model using the reflection index $(\boldsymbol{n})$ and the extinction coefficient $(\boldsymbol{k})$ for each layer listed in Table 3.6. Due to the large variability of the tantalum lattice structure depending on the sputter procedure, the parameters for the tantalum layer were experimental specified with the best fit of Psi and Delta based on the literature value ${ }^{10}$.

\footnotetext{
VI The anodic $\mathrm{Ta}_{2} \mathrm{O}_{5}$-layers were measured with the ellipsometer (EP3 View, Accurion, Germany) at the wavelength of $658 \mathrm{~nm}$ in the Department of Biomaterials in the Institute for Bioprocessing and Analytical Measurement Techniques (IBA). The ODTS coating thickness was determined with the ellipsometer (EP3 View V260, Nanofilm Technologie, Germany) at the wavelength of $532 \mathrm{~nm}$ in the research group of Prof. Dr. A. Janshoff in the Institute for Physical Chemistry at the University of Göttingen.
} 
Table 3.6. Refractive index $(n)$ and extinction coefficient $(k)$ of each layer for the analysis model.

\begin{tabular}{cccc}
\hline layer & refractive index $(\boldsymbol{n})$ & extinction coefficient $(\boldsymbol{k})$ & reference \\
\hline $\begin{array}{c}\mathrm{Ta} \\
(\boldsymbol{\lambda}=532 \mathrm{~nm})\end{array}$ & 2.92 & 3.33 & experimental value \\
$\mathrm{Ta}$ & & & \\
$(\boldsymbol{\lambda}=658 \mathrm{~nm})$ & 2.92 & 2.13 & 76,127 \\
$\mathrm{Ta}_{2} \mathrm{O}_{5}$ & 2.22 & 0 & 128 \\
ODTS & 1.46 & 0 & \\
air & 1.00 & 0 & \\
\hline
\end{tabular}

For the ellipsometry measurements, all used equipment and software were supplied by Accurion $\mathrm{GmbH}$ in Germany.

\subsubsection{Atomic Force Microscopy: Surface Topology and Roughness}

Atomic force microscopy (AFM) is an important and widely used analysis method to determine the surface topology and surface chemistry. AFM can highly resolve the scanned surface almost at the scale of the atomic level. As a standard analytical technique, AFM is described in detail concerning its working principle, the physiochemical background and its applications in textbooks ${ }^{129-130}$. In this study, AFM was applied to topologically scan and image the surface of the anodic $\mathrm{Ta}_{2} \mathrm{O}_{5}$ layers as well as that of the ODTS coating.

\section{Setup}

An atomic force microscope, NanoWizard ${ }^{\circledR} 4$ NanoScience, was placed on a vibration isolation system (Accurion $\mathrm{GmbH}$, Germany) equipped with a JPK TopViewOptics ${ }^{\mathrm{TM}}$ camera. This setup was located in a JPK acoustic enclosure on a JPK base. For specimens with either a $\mathrm{Ta}_{2} \mathrm{O}_{5}$ surface or a silane surface, AFM cantilevers with a $\mathrm{Si}_{3} \mathrm{~N}_{4}$-tip (NCHR-50, Pointprobe ${ }_{-}^{\Theta_{-}}$ Silicon SPM-Sensor, non-contact mode, Nanoworld, Germany) were used.

The AFM preparation and operation were performed with the SPM software (software v.6).

\section{Procedure}

The specimen was attached to a glass slide and placed on the sample stage. The measurement was prepared according to the user manual ${ }^{131}$ for the NanoWizard ${ }^{\circledR}$ devices. In case of the specimens with a $\mathrm{Ta}_{2} \mathrm{O}_{5}$ surface, the cantilever end was controlled with the TopViewOptics ${ }^{\mathrm{TM}}$, which was positioned right over the anodic $\mathrm{Ta}_{2} \mathrm{O}_{5}$ area. 
For a $\mathrm{Ta}_{2} \mathrm{O}_{5}$ surface, the tapping mode $(\mathrm{AC} \text { mode })^{131}$ in air was set in the software for measurement. For an ODTS surface, the quantitative imaging $\left(\mathrm{QI}^{\mathrm{TM}}\right)$ mode $^{131}$ in air was selected due to the strong repulsive interaction between the hydrophobic surface and the hydrophilic cantilever tip. ${ }^{132}$ The scanning was performed according to the user manual ${ }^{131}$ with the following parameter settings for the $\mathrm{Ta}_{2} \mathrm{O}_{5}$ surface and for the ODTS surface respectively.

Table 3.7. Parameter settings for the AFM imaging of a $\mathrm{Ta}_{2} \mathrm{O}_{5}$ surface (left) and for that of an ODTS surface (right).

\begin{tabular}{|c|c|c|c|}
\hline \multicolumn{2}{|c|}{ AC mode } & \multicolumn{2}{|c|}{$\mathrm{QI}^{\mathrm{TM}}$ mode } \\
\hline I Gain & $300 \mathrm{~Hz}$ & Cantilever & $\mathrm{NCH}-\mathrm{AC}$ \\
\hline Set point & $0.598 \mathrm{~V}$ & Set point & $13.24 \mathrm{nN}$ \\
\hline Z-range & $5 \mu \mathrm{m}$ & Z-length & $0.172 \mu \mathrm{m}$ \\
\hline Line rate & $1 \mathrm{~Hz}$ & Speed & $64.18 \mu \mathrm{m} / \mathrm{s}$ \\
\hline
\end{tabular}

The topographical information was repeatedly collected with different specimens and various regions of interest with a size of $20 \mu \mathrm{m} \times 20 \mu \mathrm{m}$.

\section{Data Processing}

With the software, JPK data processing (Version 6.0.64), the topographical information of each scanned region was processed to an image. The data processing was operated according to the software manual ${ }^{133}$. Each image and its roughness were obtained through following operations: (i) The height data were corrected through a line-by-line fitting with a polynomial function. (ii) The root-mean-square value of roughness $\left(\boldsymbol{R}_{\mathrm{RMS}}\right)$ was determined with the histogram tool in the software.

All applied equipment, accessories and softwares for the AFM measurement were provided by JPK instruments in Germany, when no other assignment is mentioned.

\subsubsection{Contact Angle Determination: Surface Hydrophobicity}

Contact angle measurement ${ }^{39}$ is a method for characterizing the surface chemistry. The contact angle describes the wettability of a surface by a liquid in a second fluid phase (gas or another immiscible liquid). In the case of a water droplet on a surface, low contact angle values indicate surface hydrophilicity and high contact angle values indicate hydrophobicity. ${ }^{41}$ The quantitative correlation between the contact angle and the interfacial tensions is described by the Young's equation (Eq. 2.1).

Here, the 3-phase-circumstances under investigation were (i) a water droplet on the anodic $\mathrm{Ta}_{2} \mathrm{O}_{5}$ surface in air, (ii) a water droplet on the ODTS surface in air and (iii) a water droplet on the ODTS surface in oil. 


\section{Setup}

In this study, the sessile drop method was used with the tangent leaning fitting to determine the contact angle. The same measurement principle was applied for the droplet geometry determination with the imaging system constructed for the LV-EWOD-EIS system (Chapter 3.3.1).

The measurement setup was a commercial contact angle measurement system (OCA 20) and its software (SCA 20). The equipment consists of a camera, a back light, a dosing system and a sample table. The software controls the droplet dosing, the baseline setting and the contact angle determination with the tangent leaning fitting method. The equipment and the software were supplied by the company, Dataphysics instruments in Germany.

\section{Procedure}

The specimens for the three measurement circumstances were (i) an anodized electrode substrate after cleaning with the acid piranha solution (Chapter 3.1.1, cleaning step 1), (ii) an electrode substrate with a $\mathrm{Ta}_{2} \mathrm{O}_{5}$ initial layer $\left(\boldsymbol{U}_{\text {anod }}=30 \mathrm{~V}\right)$ and an ODTS top layer and (iii) a fabricated LV-EWOD electrode immersed in tetradecane (oil) in a glass cuvette. For all specimens, the measurements were performed according to the user manual ${ }^{134}$ with the setting listed in Table 3.8.

Table 3.8. Parameters for the contact angle measurements.

\begin{tabular}{cc}
\hline measurement parameter & setting \\
\hline droplet size & $3 \mu \mathrm{L}$ \\
droplet solution & deionized water \\
calculation & tangent leaning \\
number of data points & 15 \\
number of measured sites & 3 \\
\hline
\end{tabular}

Each circumstance was measured on three sites of the specimen surface. For each measurement, the mean contact angle was determined by averaging the left and right contact angle. Fifteen data points were recorded for the statistic mean value and standard deviation. 


\subsubsection{Measurement of Contact Angle Hysteresis: Surface Friction}

Contact angle hysteresis $(\mathrm{CAH})^{39,} 135$ indicates the surface resistance against drop motion. $\mathrm{CAH}$ is defined as the difference between an advancing contact angle $\left(\boldsymbol{\theta}_{\text {adv }}\right)$ and a receding contact angle $\left(\boldsymbol{\theta}_{\text {rec }}\right)$ as below:

$$
\boldsymbol{C A H}=\boldsymbol{\theta}_{a d v}-\boldsymbol{\theta}_{\text {rec }}
$$

These two contact angles can be determined with two measurement techniques: (i) dynamic sessile drop method and (ii) tilting method.

\section{Two Measurement Techniques}

In case of the dynamic sessile drop method ${ }^{18}$, the volume of a sessile drop is continuously varied. The advancing angle is measured as the maximal contact angle, while the drop volume is increased, before its contact area to the substrate advances. Reversely, the receding angle is measured as the minimal contact angle, while the drop volume is decreased, before the contact area recedes.

In case of the tilting method ${ }^{20}$, the measurement system with a sessile drop resting on the specimen is tilted to bring the drop into motion. While the drop moves, its larger contact angle on one site of the interfacial intersection is measured as the advancing contact angle. Its smaller contact angle on the other site is the receding contact angle.

In this study, the two methods were used for two different measurement circumstances: (i) For a water droplet on the ODTS surface in air, the CAH was determined with the dynamic sessile drop method. (ii) For a water droplet on the ODTS surface in an oil environment, the tilting method was applied to determine the $\mathrm{CAH}$.

Under the circumstance (i) in air, the large surface friction hindered the droplet motion despite of the system tilting to a large extent. This $\mathrm{CAH}$ could not be measured with the tilting method. Under the circumstance (ii) in the oil surrounding, the needle for dispensing and reverse dispensing could not be kept inserted in the drop. This made the $\mathrm{CAH}$ of this circumstance not measurable with the dynamic sessile drop method. Therefore, the CAHs of the two circumstances were determined using different measurement techniques.

\section{Setup}

The contact angle system (OCA 20) and the software (SCA 20) used for the CAH measurement were the same equipment and software involved in the contact angle determination (Chapter 3.2.5).

\section{Procedure}

Both $\mathrm{CAH}$ measurements were performed according to the user manual ${ }^{134}$. The specimen for the measurement circumstance (i) was an electrode substrate with a $\mathrm{Ta}_{2} \mathrm{O}_{5}$ initial layer $\left(\boldsymbol{U}_{\text {anod }}=30 \mathrm{~V}\right)$ and an ODTS top layer. The advancing and the receding contact angle were 
successively determined using the dynamic sessile drop method with the parameters listed in Table 3.9. The CAH was calculated with Eq. 3.2.

Table 3.9. Parameters for the CAH measurements with the dynamic sessile drop method.

\begin{tabular}{cc}
\hline measurement parameter & setting \\
\hline mode & needle in \\
$\begin{array}{c}\text { velocity of dispensing and } \\
\text { reverse dispensing } \\
\text { droplet solution } \\
\text { calculation } \\
\text { number of data points } \\
\text { number of measured sites }\end{array}$ & $3 \mu \mathrm{L} / \mathrm{s}$ \\
& 15 advancing contact angles, 15 receding contact angles \\
& 3 \\
\hline
\end{tabular}

The specimen for the measurement circumstance (ii) was a fabricated LV-EWOD electrode immersed in tetradecane (oil) in a glass cuvette. The advancing and the receding contact angle were determined with the tilting method. Besides the tilting of the sample stage, the measurement parameter setting was identical to that for the contact angle determination (Table 3.8). The CAH was calculated with Eq. 3.2. 


\subsection{Integrated LV-EWOD-EIS Systems}

A novel integrated LV-EWOD-EIS system associated with optical imaging was established within this doctoral research for the online investigation of the EWOD effect by EIS and imaging. Its setup, as demonstrated in Figure 3.12, consists of four modules: (I) measurement cell, (II) electroanalytical instrument, (III) optical imaging instrument and (IV) software. This modular setup enables versatile investigation and application opportunities through its adaptability to the individual measurement tasks.

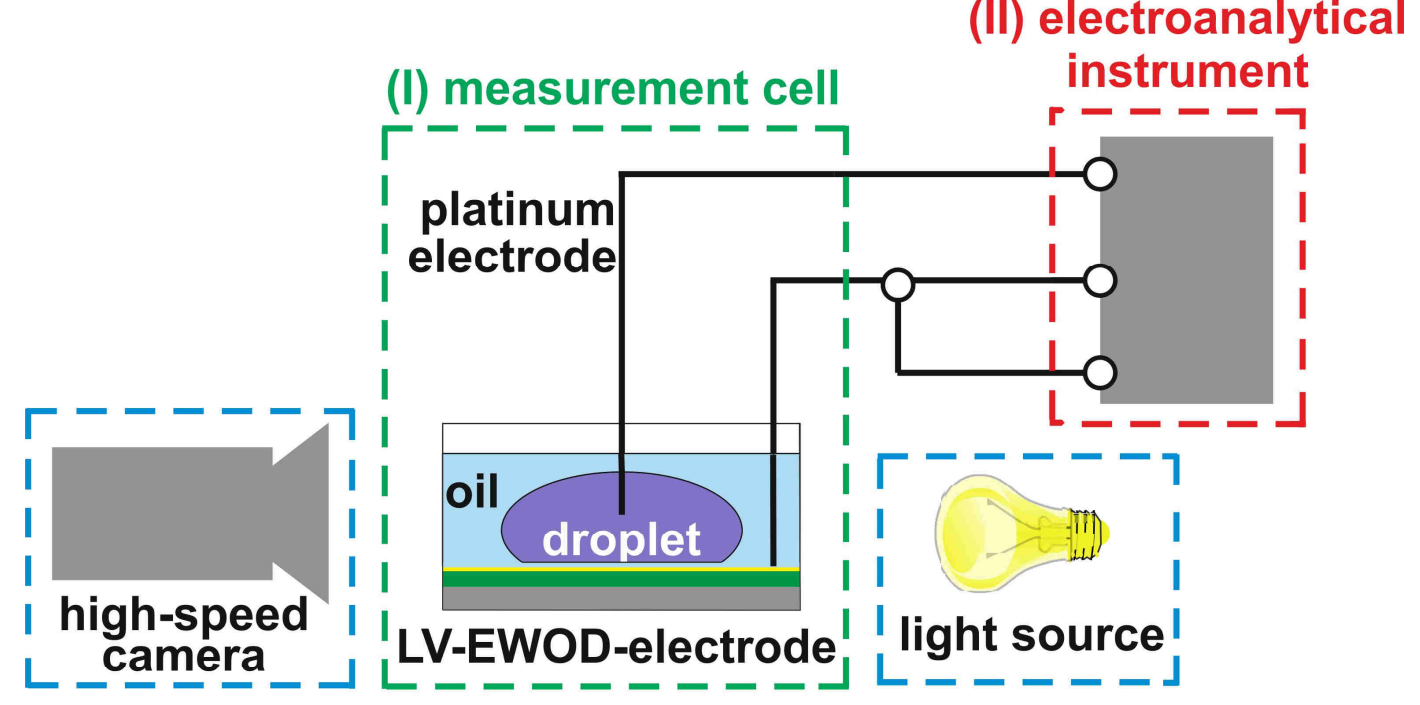

(III) optical imaging instrument

(IV) software

Figure 3.12. Schematic setup of the integrated LV-EWOD-EIS system associated with optical imaging. Modular setup with (I) measurement cell, (II) electroanalytical instrument, (III) optical imaging instrument and (IV) software.

In this doctoral research, two measurement principles were applied: EWOD integrated with EIS in frequency domain (Chapter 2.1.2.1) and EWOD integrated with dynamic EIS (Chapter 2.1.2.2). These two measurement principles were implemented by two experimental setups consisting of the four modules. They share the measurement cell and the optical imaging instrument but utilize individual electroanalytical instruments and are controlled by different software programs.

In this section, the four modules composing this integrated system are individually presented in the beginning (Chapter 3.3.1). For each module, their components are introduced with the focus on the functionality and the specification. The two subsequent chapters are devoted to one measurement application with frequency-domain EIS (Chapter 3.3.2) and to another with dynamic EIS (Chapter 3.3.3). Each measurement application is described in detail concerning the measurement setup, the procedure and the data processing. 


\subsubsection{Integrated System with Modular Setup}

\section{Measurement Cell}

Concerning the role in the integrated LV-EWOD-EIS system, the measurement cell contains the object under investigation: an aqueous saline drop on the LV-EWOD electrode surrounded by an immiscible oil (tetradecane). In principle, the centerpiece is an electrochemical cell in oil with the LV-EWOD electrode as the working electrode, the droplet as the electrolyte solution and a platinum electrode as the counter electrode.
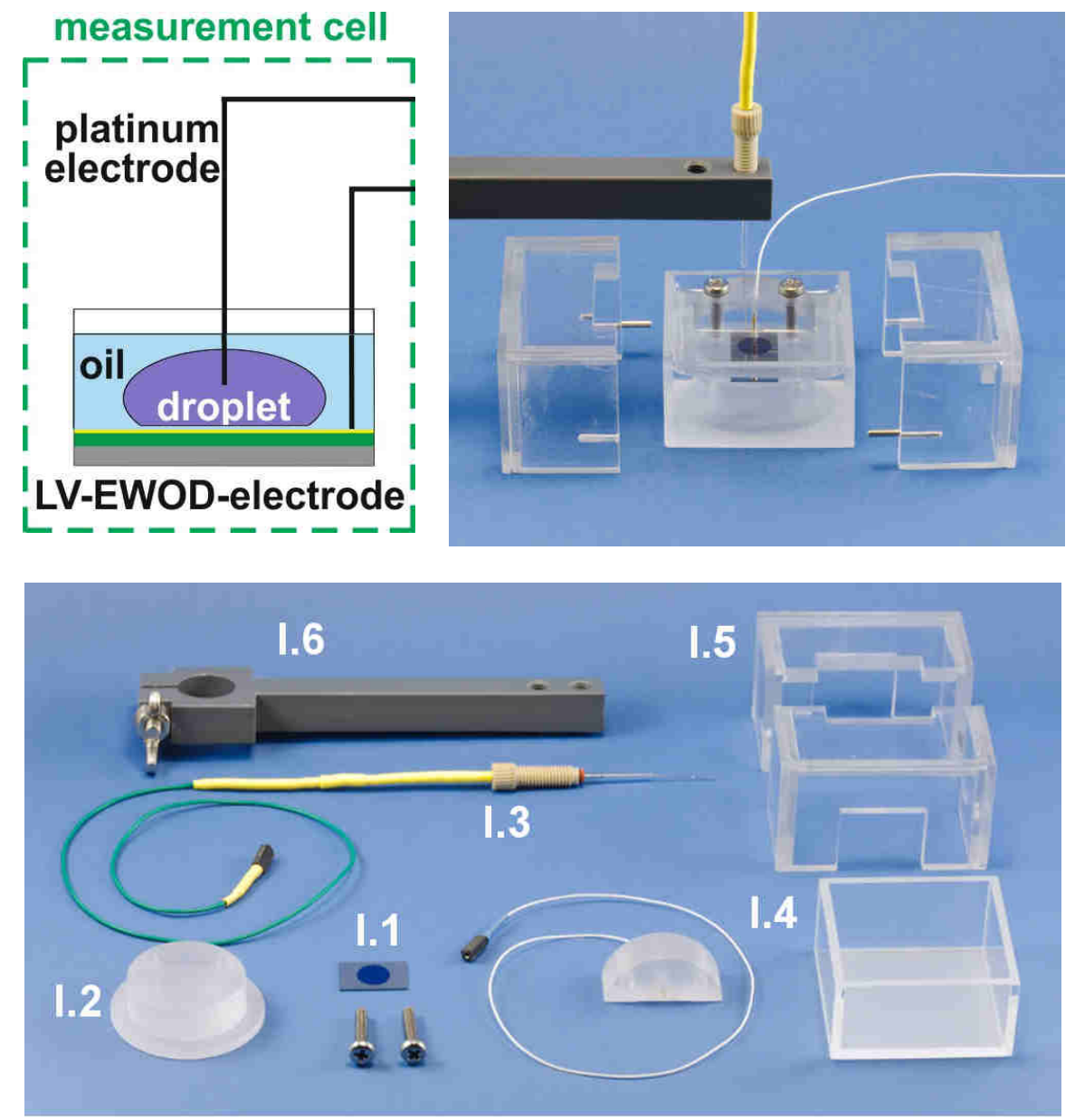

Figure 3.13. Measurement cell setup and its disassembled components: a LV-EWOD electrode (I.1), an electrode holder (I.2), a platinum electrode (I.3), a glass cuvette (I.4), a transparent hood (I.5) and a holding bar (I.6).

The measurement cell setup and its disassembled hardware components are shown in Figure 3.13. The LV-EWOD electrode (I.1) was fabricated according to Chapter 3.1. The electrode holder (I.2), the platinum electrode (I.3), the transparent hood (I.5) and the holding bar (I.6) were developed and fabricated in-house. Technical drawings are enclosed in Appendix 7.7 (Li02, Li03, Li04, Li05, Li06, Li07, Li08). The glass cuvette (I.4) was a high precision glass cell made for optical spectroscopy and supplied by Hellma Analytics, Germany. The functionality and specification of each component are listed in the following table. 
Table 3.10. Functionality and specification of the hardware components in the measurement cell.

\begin{tabular}{|c|c|}
\hline component name & functionality and specification \\
\hline $\begin{array}{l}\text { LV-EWOD electrode } \\
\text { (I.1) }\end{array}$ & $\begin{array}{l}\text { The electrode coating consists of an anodic } \mathrm{Ta}_{2} \mathrm{O}_{5} \text { initial layer } \\
\text { fabricated at } 30 \mathrm{~V} \text { and an ODTS top layer. The electrode was } \\
\text { conditioned in tetradecane. (Chapter } 3.1 \text { ) } \\
\text { It enables the EWOD effect to be induced by applying low voltages, } \\
\text { which is the foundation for the integrated EWOD excitation and EIS } \\
\text { measurement using one signal. }\end{array}$ \\
\hline $\begin{array}{l}\text { electrode holder } \\
\text { (I.2) }\end{array}$ & $\begin{array}{l}\text { The electrode consists of a bottom part, an upper part and two } \\
\text { screws. } \\
\text { The bottom part has an indentation for fixing an EWOD electrode. } \\
\text { The upper part processes a spring pin and a thin cable for connecting } \\
\text { an electrical measurement device. } \\
\text { With two screws, the electrode holder is mounted with an EWOD } \\
\text { electrode positioned on the bottom part and half covered by the } \\
\text { upper part. The EWOD electrode can be connected to the electrical } \\
\text { measurement device }\end{array}$ \\
\hline $\begin{array}{l}\text { platinum electrode } \\
\text { (I.3) }\end{array}$ & $\begin{array}{l}\text { The platinum electrode served as the counter electrode in the } \\
\text { electrical measurements. } \\
\text { It consists of a pure platinum wire with a diameter of } 0.23 \mathrm{~mm} \text { (purity } \\
\text { proven by energy-dispersive X-ray spectroscopy) soldered on a thin } \\
\text { cable and fixed in a glass capillary within a holder. The platinum } \\
\text { electrode can be fixed on the holding bar (I.6) on a sample stage to } \\
\text { be set at a proper distance to the EWOD electrode. }\end{array}$ \\
\hline $\begin{array}{l}\text { glass cuvette } \\
(1.4)\end{array}$ & $\begin{array}{l}\text { The glass cuvette has a size of } 40 \mathrm{~mm}(\mathrm{I}) \times 40 \mathrm{~mm}(\mathrm{w}) \times 20 \mathrm{~mm}(\mathrm{~h}) \text {. } \\
\text { It serves as a container for tetradecane as the fluid surrounding for } \\
\text { the EWOD experiment. Its transparent glass walls permit high } \\
\text { transmission of light and support the good image quality in the optical } \\
\text { measurement. }\end{array}$ \\
\hline
\end{tabular}




\begin{tabular}{|l|l|}
\hline transparent hood & $\begin{array}{l}\text { The transparent hood serves mainly to minimize oil evaporation and } \\
\text { contaminations, such as dusts, from the ambient air. } \\
\text { It consists of two assembled parts for positioning around the glass } \\
\text { cuvette (I.4). Each part has an opening on the side to let the light } \\
\text { beam pass through the cuvette for optical detection. Another } \\
\text { opening on the top side enables the positioning of the platinum } \\
\text { electrode in a short distance to the EWOD electrode. }\end{array}$ \\
\hline (I.6) & $\begin{array}{l}\text { The holding bar is fixed on the sample stage to enable a proper } \\
\text { configuration of the counter electrode, working electrode and } \\
\text { reference electrode (if used). }\end{array}$ \\
\hline sample stage & $\begin{array}{l}\text { The sample stage supports the measurement cell and facilitates its } \\
\text { independent position adjustment in the x-, y- and z- direction. It can } \\
\text { be adjusted in the horizontal plane to control and avoid the stage } \\
\text { tilting. } \\
\text { It consists of following components: } \\
\text { a microscope table for the position adjustment in the x- and } \\
\text { a lirection } \\
\text { a base with four height-adjustable pillars and a spirit level for precise } \\
\text { setting in the horizontal plane }\end{array}$ \\
\hline
\end{tabular}

\section{Electroanalytical Instruments}

The simultaneous LV-EWOD excitation and EIS measurement were achieved using the electroanalytical instrument. It consists of a digital USB oscilloscope, a transimpedance amplifier and a power source. The oscilloscope fulfills two tasks: It serves as an arbitrary waveform generator and possesses two measurement channels, Channel 1 and Channel 2, for simultaneously recording the applied signal and the signal response in units of voltage. The transimpedance amplifier ${ }^{30}$ converts the signal response in current to voltage. The amplification can be adjusted by setting the value of a feedback resistor, so that the signal response can be recorded by the oscilloscope. The signal generation, the data acquisition and the response analysis are controlled by software programs written in Python (Chapter 3.3.1.IV). 
The LV-EWOD-EIS system was implemented using two hardware setups (Table 3.11), one for measurements using frequency-domain EIS (Chapter 3.3.2) and the other using dynamic EIS (Chapter 3.3.3).

Table 3.11. Hardware components of two instrumental setups corresponding to measurement applications with frequency-domain EIS and with dynamic EIS respectively.

\begin{tabular}{|c|c|c|}
\hline $\begin{array}{l}\text { hardware } \\
\text { components }\end{array}$ & frequency-domain EIS & dynamic EIS \\
\hline oscilloscope & $\begin{array}{l}\text { Handyscope HS3 (Tiepie } \\
\text { engineering, Netherlands) }\end{array}$ & $\begin{array}{l}\text { Handyscope HS5 (Tiepie } \\
\text { engineering, Netherlands) }\end{array}$ \\
\hline $\begin{array}{l}\text { transimpedance } \\
\text { amplifier }\end{array}$ & $\begin{array}{l}\text { in-house developed and } \\
\text { fabricated front-end } \\
\text { transimpedance amplifier and } \\
\text { feedback resistor }\end{array}$ & $\begin{array}{l}\text { variable gain high speed current } \\
\text { amplifier DHPCA-100 (FEMTO } \\
\text { Messtechnik GmbH, Germany) }\end{array}$ \\
\hline power supply & $\begin{array}{l}\text { in-house fabricated power supply, } \\
\text { output: } \pm 12 \mathrm{~V}\end{array}$ & $\begin{array}{c}\text { Traco Power (Traco electronic AG, } \\
\text { Germany), output: DC } \pm 15 \mathrm{~V}\end{array}$ \\
\hline
\end{tabular}

Sufficient measurement accuracy was confirmed for both setups by calibration with standard $\mathrm{RC}$ combinations.

In comparison to the conventional EIS equipment based on a commercial potentiostat (ref. Chapter 3.2.1), the two in-house developed setups possess several advantages: programmable input signal for combination with EWOD excitation, extended voltage measurement range, low-cost and small size.

\section{Optical Imaging Instrument}

In parallel to the electrical analysis, EWOD effect was investigated by the online optical detection of the droplet geometry parameters including contact angle and contact diameter. The optical measurement was implemented with a high-speed imaging instrument.

The hardware setup consists of a high-speed camera (Pike F-032C, Allied Vision Technologies $\mathrm{GmbH}$, Germany) with an associated objective (Micro-NIKKOR $105 \mathrm{~mm} \mathrm{f} / 2.8$, Nikon, Japan) and a simple LED (light-emitting diode) lamp with a dimmer as back light. The specification of the high-speed camera is listed in Table 3.12. 
Table 3.12. Specification of the Pike high-speed camera. ${ }^{136}$

\begin{tabular}{cc}
\hline parameter & technical data \\
\hline camera type & video graphic array camera \\
sensor technique & CCD \\
sensor type & KAI-0340 \\
cell size $/ \mu m^{2}$ & $7.4 \times 7.4$ \\
interface & IEEE 1394b Fire Wire $-800 \mathrm{Mb} / \mathrm{s}$ \\
\hline
\end{tabular}

For the measurement applications in this doctoral research, the imaging instrument recorded images at a high rate between 715 and 720 frames per second for a defined observation window with a length of 640 pixels and a height of 100 pixels. This observation window was set at the maximal zoom of the objective and covered the entire intersect region of a $3 \mu \mathrm{L}$ droplet on the EWOD electrode in oil (Figure 3.14).

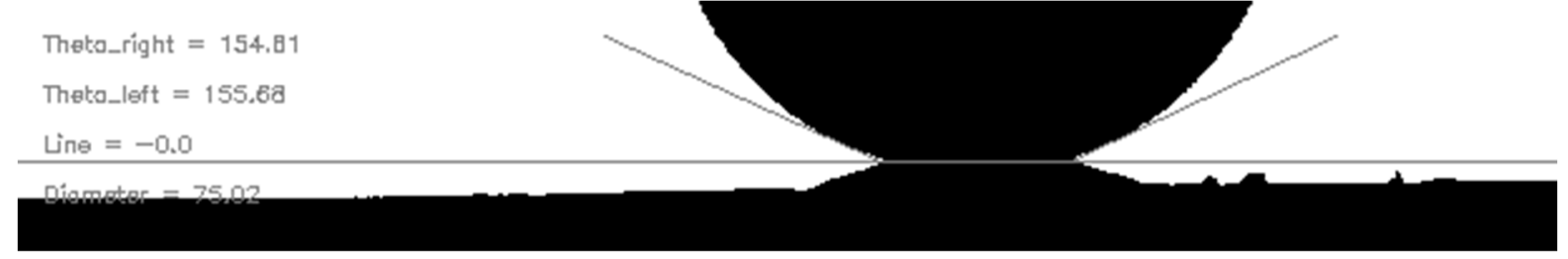

Figure 3.14. Recorded image of the intersection region of a $3 \mu \mathrm{L}$ droplet on the EWOD electrode. Image analysis with the baseline and the two tangent lines yields the right and the left contact angles as well as the diameter of the contact area.

The image acquisition and analysis were controlled and implemented with software programs written in Python (Chapter 3.3.1.IV).

The high-speed imaging instrument in combination with the software programs possess the functionalities comparable to a commercial contact angle measurement system (ref. Chapter 3.2.5) for setting base line and image contrast as well as for measuring the contact angle and contact area. Moreover, it is advantageous in the high-speed image recording, the programmed simultaneous measurements with electric analysis and low cost. 


\section{Software Programs ${ }^{\text {VII }}$}

To simultaneously implement the EWOD excitation, the EIS measurement and the optical imaging analysis, software programs were developed to achieve a centralized control of hardware units, automatic data acquisition and basic data processing.

Four software scripts were written in-house in Python 2.7 (Python Software Foundation) for the two measurement applications: one with frequency-domain EIS (Chapter 3.3.2) and the other with dynamic EIS (Chapter 3.3.3). The four program codes are enclosed on the webpage ${ }^{137}$ of Institute for Bioprocessing and Analytical Measurement Techniques (IBA). Their main functionalities and applications are summarized below:

\section{Program 1: acquiretheta3}

This program serves to prepare the image acquisition in both measurement applications (Chapter 3.3.2 and 3.3.3). It enables the baseline setting and tilting in the observation window to fit the droplet-electrode-intersection (Figure 3.14) and the regulation of the image contrast. Moreover, the platinum wire diameter was measured with this program for determining the actual pixel-to-millimeter ratio used for the unit-conversion in analyzing the recorded images.

\section{Program 2: Impedancecameraloop13}

This program combines the imaging with the simultaneous EWOD excitation and frequencydomain EIS for the measurement application presented in Chapter 3.3.2.

It serves to define the key parameters of a sinusoidal voltage signal concerning its frequency, amplitude, offset and signal length. The Handyscope generates the electrical signal for the EWOD excitation and acquires the EIS measurement data. Moreover, this program implements loops that enable the automation of measurements covering a wide range of frequencies and voltage amplitudes. Concerning the imaging functionality, it triggers the highspeed camera for continuous image acquisition at each frequency and voltage.

This program serves to record the applied signal and the signal response as a voltage signal and to convert the signal response in current via the given value of the feedback resistor. Based on the theory of frequency-domain EIS (Chapter 2.1.2.1), the impedance and the phase shift are determined in this program using the applied voltage signal and the current feedback. Concerning the image analysis, this program determines the contact diameter of the dropletelectrode-intersection and calculates the average contact angle from the measured left and right contact angles.

\footnotetext{
VII Program 1, 2 and 3 were mainly written by Dr. Brian Cahill (Junior Researcher Department) at IBA. Program 4 was mainly written by Jiaji Pan (former intern of Department for Analytical Measurement Techniques) at IBA.
} 


\section{Program 3: aquaretheta5}

This program supports a continuous image acquisition and analysis. It was used in parallel to the dynamic EIS measurement (Chapter 3.3.3). It is manually triggered at the measurement beginning of the dynamic EIS. It records and analyzes the droplet deformation along the staircase voltage signal with a high time resolution. It yields the contact area and the average contact angle from the measured left and right contact angles.

\section{Program 4: staircase}

In this program, the EWOD excitation and the dynamic EIS measurement are integrated using a staircase voltage signal with a square wave function on each voltage step (Figure 2.7, Chapter 2.1.2.2). Its measurement application is described in Chapter 3.3.3.

This program allows to define the frequency and the voltage amplitude of the square wave function as well as the maximal voltage of the staircase signal. The applied signal and the signal response are both recorded as voltage signals in a very high time resolution.

\subsubsection{LV-EWOD with Frequency-Domain EIS}

For investigation of the frequency dependence of the EWOD effect, the LV-EWOD-EIS system (Figure 3.12) was applied to integrate EWOD excitation, frequency-domain EIS and optical imaging. The measurements yielded the raw data for determination of the EWOD-related variables (Chapter 4.2) at different frequency $(100 \mathrm{~Hz}-1 \mathrm{MHz})$ under the voltage variation $(0.5 \mathrm{~V}-5 \mathrm{~V})$ for a $3 \mu \mathrm{L}$ droplet of a $\mathrm{KCl}$ solution $(6.25 \mathrm{mM}-200 \mathrm{mM})$.

\section{Setup}

The measurement setup consisted of the four modules of the LV-EWOD-EIS system (Figure 3.12). In Module II (electroanalytical instrument, Table 3.11), the centerpieces were the Handyscope HS3 and the in-house fabricated transimpedance amplifier (Figure 3.15). The circuit diagram of the later is enclosed in Appendix 7.6.

The transimpedance amplifier, as shown in Figure 3.15, was connected to the Handyscope HS3 via its three channels (AWG, Ch1, Ch2), and connected to the measurement cell via the working electrode (WE) and the counter electrode (CE). A feedback resistor was attached to the amplifier to set the transimpedance gain. A power supplier was connected to the backside of the transimpedance amplifier. The Handyscope HS3 and the imaging instrument (Chapter 3.3.1.III) were connected to a computer that carried out the software programs (Chapter 3.3.1.IV).

\footnotetext{
VIII The in-house fabricated transimpedance amplifier and the power supplier were provided by the Department of Analytical Measurement Techniques at IBA.
} 


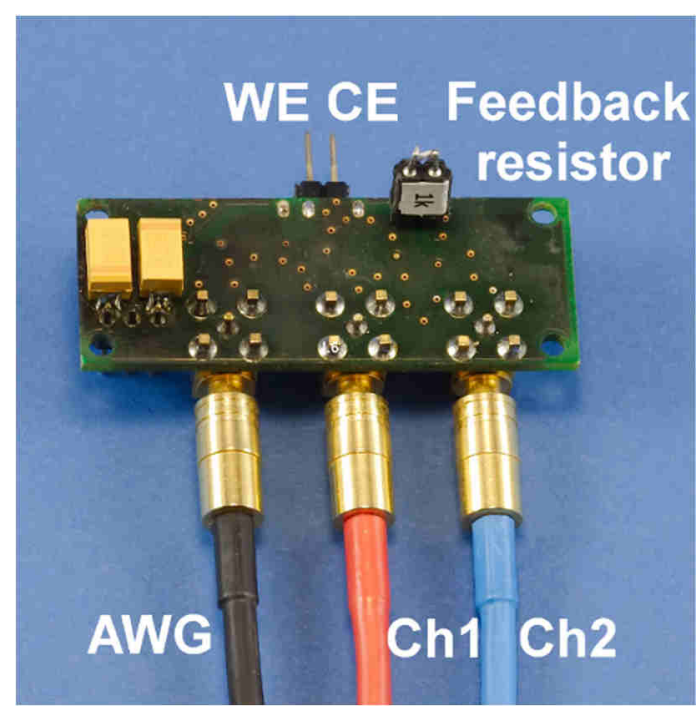

Figure 3.15. Transimpedance amplifier with a changeable feedback resistor, connections to the working electrode (WE) and the counter electrode (CE) as well as connections to the three channels of the Handyscope. AWG: arbitrary waveform generation, Ch1: Channel 1 for recording the applied signal, Ch2: Channel 2 for recording the response signal.

\section{Procedure}

First, a $3 \mu \mathrm{L}$ droplet of $\mathrm{KCl}$ solution $(6.25 \mathrm{mM}-200 \mathrm{mM})$ was placed on the LV-EWOD electrode (working electrode) in tetradecane in the measurement cell (Figure 3.13). The platinum electrode was inserted in the droplet and served as counter electrode. Both electrodes were connected to the transimpedance amplifier (Figure 3.15). Depending on the $\mathrm{KCl}$-concentration in the droplet, an appropriate feedback resistor (Table 3.13) was used in the measurement setup.

Table 3.13. $\mathrm{KCl}$ concentrations in the droplet and the correspondingly used feedback resistors.

\begin{tabular}{cc}
\hline $\mathbf{K C l}$ concentration / $\mathbf{m M}$ & resistor value / $\mathbf{\Omega}$ \\
\hline 200 & 1.203 \\
100 & 2.207 \\
50 & 6.860 \\
25 & 7.400 \\
12.5 & 14.90 \\
6.25 & 27.00 \\
\hline
\end{tabular}

Second, the Python program "acquiretheta3" (Program 1, Chapter 3.3.1 IV) was executed to prepare the imaging measurement. 
Third, the simultaneous EWOD excitation, EIS in the frequency domain and imaging measurement were conducted by using the Python program "impedancecameraloop13" (Program 2, Chapter 3.3.1 IV) with the following parameter setting (Table 3.14).

Table 3.14. Parameter setting for the simultaneous EWOD excitation, EIS in the frequency domain and imaging measurement.

\begin{tabular}{ccc}
\hline \multicolumn{1}{c}{ parameter } & variable label in the program code & values / unit \\
\hline $\begin{array}{c}\text { number of frames recorded per } \\
\text { amplitude and per frequency } \\
\text { initial excitation frequency } \\
\text { final excitation frequency }\end{array}$ & frames & $10-100$ \\
frequency values per decade & exfreqstart & $1000000 \mathrm{~Hz}$ \\
initial excitation amplitude & exfreqend & $100 \mathrm{~Hz}$ \\
final excitation amplitude & perdecade & 5 \\
number of amplitude values & amplitudestart & $0.5 \mathrm{~V}$ \\
amplitude increment & amplitudeend & $5 \mathrm{~V}$ \\
resistance of feedback resistor & amplitudeloops & 10 \\
\hline
\end{tabular}

The measurement series were performed in the frequency range from $1 \mathrm{MHz}$ to $100 \mathrm{~Hz}$ using a sinusoidal alternating voltage signal with an offset $\left(\boldsymbol{U}_{\mathbf{o f f s e t}}\right)$ equal to the amplitude $\left(\boldsymbol{U}_{\mathbf{p p} / \mathbf{2}}\right)$. The amplitude was varied from $0.5 \mathrm{~V}$ to $5 \mathrm{~V}$ in steps of $0.5 \mathrm{~V}$. Measurements were conducted with several EWOD electrodes and repeated three times with each electrode to ensure the reproducibility.

\section{Data Processing}

The data processing was automatically conducted by the measuring program "impedancecameraloop13" (Program 2, Chapter 3.3.1 IV) for each single measurement at each frequency and applied voltage. Based on the theory of frequency-domain EIS (Chapter 2.1.2.1), the impedimetric data were analyzed to generate the impedance spectra and to determine the capacitive resistance, the capacitance and the resistance. Through the image analysis, the contact angle and contact area were obtained. 


\subsubsection{LV-EWOD with Dynamic EIS}

To investigate the dynamics and non-linearity in the EWOD system during electrowetting and dewetting, the LV-EWOD-EIS system (Figure 3.12) served to integrate dynamic EIS, EWOD excitation and optical imaging. The time-resolved response of a $3 \mu \mathrm{L}$ droplet of the $200 \mathrm{mM}$ $\mathrm{KCl}$ solution to a staircase voltage signal (Figure 2.7, Chapter 2.1.2.2) was recorded by determining the contact angle, the contact area and the MDS-capacitance in parallel.

\section{Setup}

The measurement setup consisted of the four modules in the LV-EWOD-EIS system associated with optical imaging (Figure 3.12). As Module II (electroanalytical instrument, Table 3.11), a dynamic EIS measurement device was designed in-house and assembled by the Department of Analytical Measurement Techniques at IBA. As a closed device, it possesses a power switch, a USB-port for connection to a computer with the software programs (Chapter 3.3.1.IV) and a coaxial cable as connection to the working electrode and to the counter electrode. Inside the dynamic EIS measurement device, the internal feedback resistor on the transimpedance amplifier was set at $100 \Omega$ for the measuring condition in this study.

\section{Procedure}

First , the measurement cell (Figure 3.13) was setup with a $3 \mu \mathrm{L}$ droplet of $200 \mathrm{mM} \mathrm{KCl}$ solution between the platinum electrode (counter electrode) and the LV-EWOD electrode (working electrode) in analogy to the measurement preparation described in Chapter 3.3.2. Both electrodes were connected to the dynamic EIS measurement device.

Second, the Python program "acquiretheta3" (Program 1, Chapter 3.3.1 IV) was implemented to prepare the imaging measurement.

Third, the Python program "staircase" (Program 4, Chapter 3.3.1 IV) was started to prepare the dynamic EIS measurement device for the measurement.

Fourth, the Python program "staircase" with the following parameter setting (Table 3.15) and the Python program "acquiretheta5" (Program 3, Chapter 3.3.1 IV) were executed simultaneously.

The dynamic EIS measurements were performed for one period ( $1 \mathrm{~s}$ ) with a symmetric staircase signal consisting of 40 steps. Its maximal voltage was $6.12 \mathrm{~V}$. At each voltage step, the voltage offset was superposed with a square wave function signal. It had a frequency of $10 \mathrm{kHz}$ and a peak-to-peak amplitude in a ratio of 0.1 to the maximal voltage in the staircase signal. The EWOD excitation and measurement began with the ascending ramp followed by a descending ramp. In parallel, the high-speed camera recorded 2500 image frames at the rate about 720 frame per second. Measurements were repeated three times with several EWOD electrodes to ensure the result reproducibility. 
Table 3.15. Parameter setting for the simultaneous EWOD excitation and dynamic EIS.

\begin{tabular}{ccc}
\hline parameter & $\begin{array}{c}\text { variable label in the } \\
\text { program code }\end{array}$ & values / unit \\
\hline $\begin{array}{c}\text { max. voltage of the staircase signal } \\
\text { number of steps in the staircase signal } \\
\text { frequency of the square wave function }\end{array}$ & gen.amplitude & $6.12 / 5 \mathrm{~V}$ \\
$\begin{array}{c}\text { peak-to-peak amplitude of the square } \\
\text { wave function }\end{array}$ & steps & 40 \\
resistance of feedback resistor & Amp & 0.1 x max. voltage of \\
the staircase signal \\
\end{tabular}

\section{Data Processing}

The recorded images were automatically analyzed by the Python program "acquiretheta5" during a measurement (Chapter 3.3.1.IV). The averaged contact angle and contact area were determined in a time-resolved manner.

The data recorded in the dynamic EIS measurement were processed by Prof. Dr. Pliquett at IBA (Department for Analytical Measurement Techniques) within the funding project, "Impedanzspektroskopische Bioanalytik - schnell und hochparallel", 2016 FGR 0040. The data processing was based on the theory of the dynamic EIS (Chapter 2.1.2.2). It was implemented by using software programs written in-house in Matlab (R2011, MathWorks, USA) and enclosed on the webpage ${ }^{137}$ of IBA. The determined parameters were the MDS-capacitance and the characteristic time of the contact area change at each voltage jump in the staircase signal. 


\section{RESULTS AND DISCUSSION}

\subsection{Quality Features of Dielectric Layers in EWOD}

This section focuses on the characterization of EWOD-relevant parameters for the dielectric layers in the system under investigation, the multilayer dielectric stack (MDS). This characterization aims to specify the features of each dielectric layer with a view in three dimensions: surface, layer and material. This serves to assure the quality of the LV-EWOD electrode fabrication and to determine the resting state of the MDS without EWOD effect.

The EWOD-relevant parameters are firstly defined in Chapter 4.1.1. The parameters are successively characterized for each dielectric layer $\left(\mathrm{Ta}_{2} \mathrm{O}_{5}\right.$, ODTS and oil) individually and for the entire MDS in Chapter 4.1.2, 4.1.3, 4.1.4, as shown in the overview scheme (Figure 4.1). Lastly, conclusions from the parameter characterization are summarized in Chapter 4.1.5.

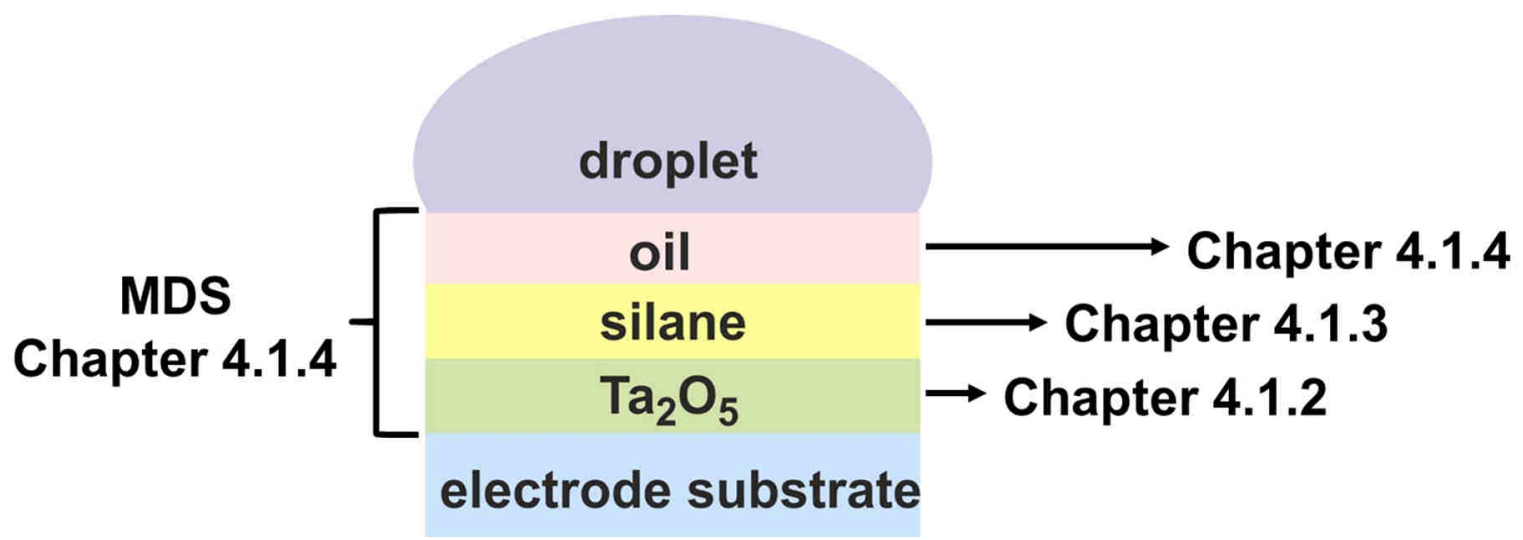

Figure 4.1. Layer model for the object under investigation (Figure 2.11) and overview of the layeroriented chapter assignment concerning characterization of EWOD-relevant parameters for each dielectric layer individually and for the entire MDS.

\subsubsection{EWOD-Relevant Parameters}

Four EWOD-relevant parameters in the Young-Lippmann equation and its transformation (Eq. 2.26) are identified in Chapter 2.2.1.1 as key to the low-voltage EWOD.

$$
\cos \theta_{\mathrm{u}}-\cos \theta_{0}=\frac{\varepsilon_{0} \varepsilon_{\mathrm{d}}}{2 \gamma_{\mathrm{lf}} d} \cdot U^{2}=\frac{1}{2 \gamma_{\mathrm{lf}}} \cdot \frac{C}{A} \cdot U^{2}
$$

These key parameters are highlighted in blue: $\boldsymbol{\theta}_{\mathbf{0}}$ stands for the contact angle of droplet in its resting or dewetted state and reflects the surface hydrophobicity, $\boldsymbol{C} / \boldsymbol{A}$ is the capacitance per unit area, $\boldsymbol{d}$ the layer thickness and $\boldsymbol{\varepsilon}_{\mathbf{d}}$ the relative permittivity of the entire dielectric layers in EWOD. 
Besides the four parameters, a fifth essential parameter for EWOD, the surface friction against droplet motion, is indirectly implied in the Young-Lippmann equation by $\cos \boldsymbol{\theta}_{\mathbf{u}}-\boldsymbol{c o s} \boldsymbol{\theta}_{\mathbf{0}}$. Due to its possible influence on the droplet wetting and dewetting process, the surface friction may affect the minimal contact angle under voltage $\left(\boldsymbol{\theta}_{\mathbf{u}}\right)$ and the reattainment of the contact angle at the resting state $\left(\boldsymbol{\theta}_{\mathbf{0}}\right)$. The surface friction can originate from the surface topology, specifically the roughness, which may lead to surface pinning and limit the change of the contact angle.

These five EWOD-relevant parameters are classified into three categories: surface, layer and material property (Table 4.1). They were determined for each dielectric material in the MDS with various surface and layer analytics or through calculation.

Table 4.1. Overview of analytic methods or calculation applied to determine the EWOD-relevant parameters for each dielectric layer in the MDS. AFM: atomic force microscopy, CAH: measurement of contact angle hysteresis, CA: contact angle measurement, CV: cyclic voltammetry, EIS: electrical impedance spectroscopy.

\begin{tabular}{|c|c|c|c|c|c|}
\hline \multirow{2}{*}{$\begin{array}{l}\text { target } \\
\text { layer } \\
\text { (liquid / } \\
\text { solid) }\end{array}$} & \multicolumn{2}{|c|}{ surface property } & \multicolumn{2}{|c|}{ layer property } & \multirow{2}{*}{$\begin{array}{c}\begin{array}{c}\text { material } \\
\text { property }\end{array} \\
\text { relative } \\
\text { permittivity }\left(\boldsymbol{\varepsilon}_{\boldsymbol{d}}\right)\end{array}$} \\
\hline & $\begin{array}{c}\text { topology } \\
\text { (roughness), } \\
\text { friction }\end{array}$ & $\begin{array}{l}\text { hydrophobicity } \\
\qquad\left(\boldsymbol{\theta}_{\mathbf{0}}\right)\end{array}$ & $\begin{array}{l}\text { capacitance } \\
\text { per unit area } \\
\qquad(\boldsymbol{C} / \boldsymbol{A})\end{array}$ & $\begin{array}{l}\text { thickness } \\
\qquad(\boldsymbol{d})\end{array}$ & \\
\hline oil & $\mathrm{CAH}$ & $\mathrm{CA}$ & CV, EIS & calculation $^{\mathrm{IX}}$ & literature value \\
\hline silane & AFM, CAH & CA & EIS & ellipsometry & \multirow{2}{*}{ calculation $\mathrm{x}$} \\
\hline $\mathrm{Ta}_{2} \mathrm{O}_{5}$ & AFM & CA & EIS & ellipsometry & \\
\hline
\end{tabular}

For the surface characterization, the topology of the two solid layers $\left(\mathrm{Ta}_{2} \mathrm{O}_{5}\right.$ and silane) was characterized by AFM (Chapter 3.2.4). The hydrophobicity of all layers was determined by means of contact angle measurement (Chapter 3.2.5). The friction against droplet motion was evaluated by measuring the contact angle hysteresis (Chapter 3.2.6) of the hydrophobic silane surface in air and in oil respectively.

IX The oil layer thickness was calculated with the transformed Eq. 2.11, $\boldsymbol{d}=\varepsilon_{\boldsymbol{d}} \cdot \varepsilon_{\mathbf{0}} \cdot \frac{\boldsymbol{A}}{\boldsymbol{C}}$, based on the measured $\boldsymbol{C} / \boldsymbol{A}$ value and the $\boldsymbol{\varepsilon}_{\boldsymbol{d}}$ value from literature.

$\mathrm{x}$ The relative permittivity of the silane layer and that of the $\mathrm{Ta}_{2} \mathrm{O}_{5}$ layer were calculated using the transformed Eq. 2.11, $\varepsilon_{\boldsymbol{d}}=\frac{\boldsymbol{d}}{\varepsilon_{0}} \cdot \frac{\boldsymbol{C}}{\boldsymbol{A}}$. The $\boldsymbol{d}$ and $\boldsymbol{C} / \boldsymbol{A}$ values were determined experimentally. 
For the layer analytics, the capacitance per unit area for each layer was obtained using EIS (Chapter 3.2.1) and the capacitive behavior of the MDS was investigated by CV (Chapter 3.2.2). The thickness of the two solid layers was measured by imaging ellipsometry (Chapter 3.2.3). The thickness of the fluid oil layer was indirectly determined with its capacitance per unit area from the EIS analysis and its relative permittivity from the literature.

The material property, relative permittivity, was specified for the $\mathrm{Ta}_{2} \mathrm{O}_{5}$ and the silane layer fabricated in this research through calculation. This is based on the correlation of this material property to the measurable layer properties, the capacitance per unit area and the layer thickness (Eq. 2.11).

In the following chapters, a layer-by-layer characterization is presented for the $\mathrm{Ta}_{2} \mathrm{O}_{5}$ layer (Chapter 4.1.2), silane layer (Chapter 4.1.3), oil layer as well as the entire MDS (Chapter 4.1.4) with the focus on their EWOD-relevant parameters.

\subsection{2. $\mathrm{Ta}_{2} \mathrm{O}_{5}$ Thin Layer with High Relative Permittivity}

As the essential component of the EWOD electrode, an anodic thin $\mathrm{Ta}_{2} \mathrm{O}_{5}$ layer should serve as a dielectric with high relative permittivity, high capacitance and high dielectric strength. It is located adjacent to the electrode substrate and is the layer onto which the silane coating binds (Figure 4.1). The $\mathrm{Ta}_{2} \mathrm{O}_{5}$ layer was fabricated through anodization (Chapter 3.1.2).

\subsubsection{Homogeneous Smooth Hydrophilic $\mathrm{Ta}_{2} \mathrm{O}_{5}$ Surface}

A representative $\mathrm{Ta}_{2} \mathrm{O}_{5}$ layer, which was formed through anodization at $30 \mathrm{~V}$, is shown to be a homogeneous surface in the AFM image (Figure $4.2 \mathrm{a}$ ).

(a) $U_{\text {anod }}=30 \mathrm{~V}, R_{\mathrm{RMS}}=0.31 \mathrm{~nm}$

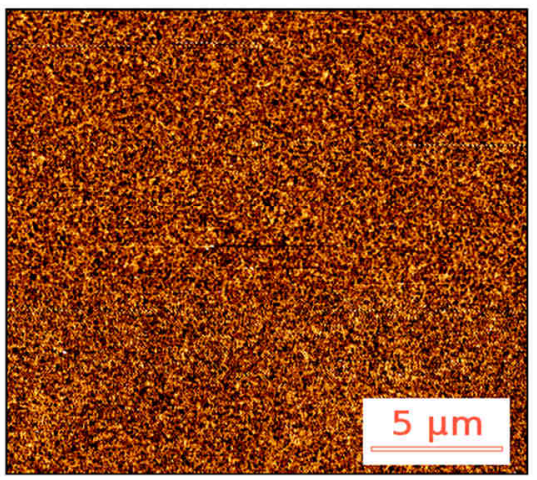

(b) $U_{\text {anod }}=50 \mathrm{~V}, R_{\mathrm{RMS}}=0.47 \mathrm{~nm}$

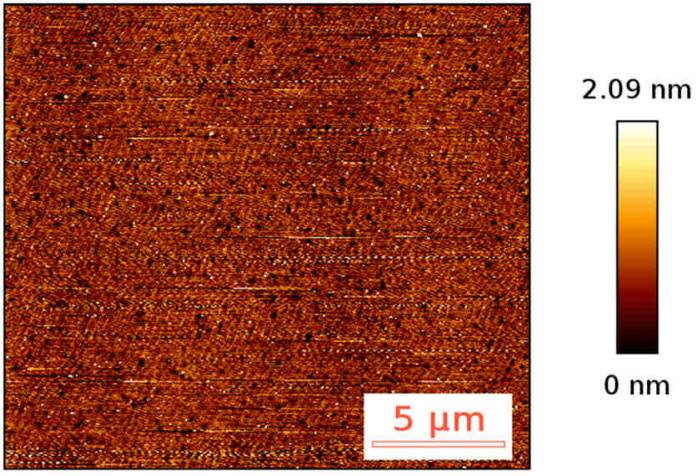

Figure 4.2. AFM images with their color-height correlations for (a) anodic $\mathrm{Ta}_{2} \mathrm{O}_{5}$ layer $\left(U_{\text {anod }}=30 \mathrm{~V}\right)$ with a root-mean-square roughness of $0.31 \mathrm{~nm}$, (b) anodic $\mathrm{Ta}_{2} \mathrm{O}_{5}$ layer $\left(U_{\text {anod }}=50 \mathrm{~V}\right)$ with a root-mean-square roughness of $0.47 \mathrm{~nm}$. 
Besides the homogeneity, this surface was very smooth. Its root-mean-square roughness $\left(\boldsymbol{R}_{\mathbf{R M S}}\right)$ of $0.31 \mathrm{~nm}$ is negligible in comparison to its layer thickness of $59 \mathrm{~nm}$ (Figure 4.5). Moreover, homogeneous surfaces with a comparable smoothness were observed for the other anodic $\mathrm{Ta}_{2} \mathrm{O}_{5}$ layers (e.g. $\boldsymbol{U}_{\text {anod }}=50 \mathrm{~V}$, Figure $4.2 \mathrm{~b}$ ) as well. This suggests anodization to be an appropriate method for fabricating $\mathrm{Ta}_{2} \mathrm{O}_{5}$ layer with a homogeneous and smooth surface. This appropriate surface topology may benefit a homogeneous and smooth subsequent coating of silane monolayer. In comparison, De Palma et al. ${ }^{116}$ reported a root-mean-square roughness of $0.8 \mathrm{~nm}$ for a dry-cleaned native $\mathrm{Ta}_{2} \mathrm{O}_{5}$ surface. Anodized $\mathrm{Ta}_{2} \mathrm{O}_{5}$ with a mean roughness of $0.6 \mathrm{~nm}$ was used by other researchers as initial layer for a thin fluoropolymer coating for low-voltage EWOD ${ }^{72}$.

Furthermore, the $\mathrm{Ta}_{2} \mathrm{O}_{5}$ surface after the anodization was thoroughly wetted by water. The water contact angle in air on the $\mathrm{Ta}_{2} \mathrm{O}_{5}$ surface was measured to be $9^{\circ}$ (Table 4.2 for comparison of the surface property between $\mathrm{Ta}_{2} \mathrm{O}_{5}$ and ODTS). This reveals the $\mathrm{Ta}_{2} \mathrm{O}_{5}$ surface to be very hydrophilic.

\subsubsection{2. $\mathrm{Ta}_{2} \mathrm{O}_{5}$ Layer Thickness}

In the optical image (Figure 4.3), the anodic $\mathrm{Ta}_{2} \mathrm{O}_{5}$ layers show different colors (dark red to bright blue) depending on the anodization voltage $(20-50 \mathrm{~V})$. The color is not a material property of $\mathrm{Ta}_{2} \mathrm{O}_{5}$, since $\mathrm{Ta}_{2} \mathrm{O}_{5}$ is transparent as bulk material and its extinction coefficient equals zero.

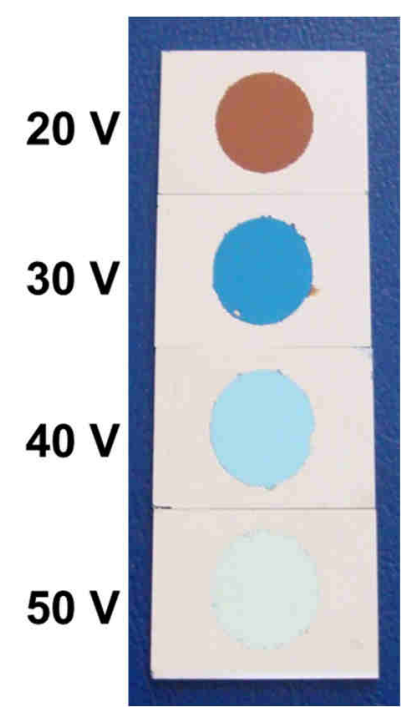

Figure 4.3. Optical image of the electrode substrates with a $\mathrm{Ta}_{2} \mathrm{O}_{5}$ layer anodized in the round middle area at various voltages $(20-50 \mathrm{~V})$.

The color results from interference ${ }^{138}$, an optical effect occurring in a transparent thin film with an interference thickness $(\mathrm{nm}-\mathrm{sub} \mu \mathrm{m})$ under visible light. Examples in nature of how thin films cause structural color are many butterfly wings and bird feathers ${ }^{139}$. It has diverse technical applications, such as interferometry ${ }^{138}$ for optical analytics, antireflection coatings ${ }^{138}$, interferometric chemical, biochemical sensors ${ }^{140}$ or colorimetric humidity sensors ${ }^{141-142}$. 
Here, the $\mathrm{Ta}_{2} \mathrm{O}_{5}$ layer functions as an interference layer, when visible light partially reflects on its upper interface to air or silane and partially on its lower interface to the reflective tantalum substrate. In general, the reflected intensity is enhanced at certain wavelengths and attenuated at other wavelengths due to interference. The $\mathrm{Ta}_{2} \mathrm{O}_{5}$ layer thickness determines the interference pattern for the reflected light and thus the observable color. Based on this, the interference colors, as reported by L. Young ${ }^{76}$, were successfully used for determination of the thickness of the oxide films on tantalum.

Since the interference-based divergence in color can mirror the thickness difference of the interference layer in $\mathrm{nm}$ range, the uniform color of each anodized area (Figure 4.3) indicates the thickness homogeneity of the $\mathrm{Ta}_{2} \mathrm{O}_{5}$ layer fabricated through anodization.

The precise thickness of the $\mathrm{Ta}_{2} \mathrm{O}_{5}$ layers anodized at 20,30, 40 and $50 \mathrm{~V}$ was determined by means of ellipsometry (Chapter 3.2.3) and its correlation with the anodization voltage was studied. In the $\Delta / \Psi$ trajectory (Figure 4.4 ), the representative data point for each layer $\left(\boldsymbol{U}_{\text {anod }}=20-50 \mathrm{~V}\right)$ in red locates close on the theoretical thickness curve in black, which was calculated with the layer stack model (Chapter 3.2.3, Table 3.6). This evinces the significant difference of layer thickness for various anodization voltages. Additionally, this confirms the validity of the layer stack model and its related optical parameters, the refractive index and the extinction coefficient, for tantalum and for the anodic $\mathrm{Ta}_{2} \mathrm{O}_{5}$.

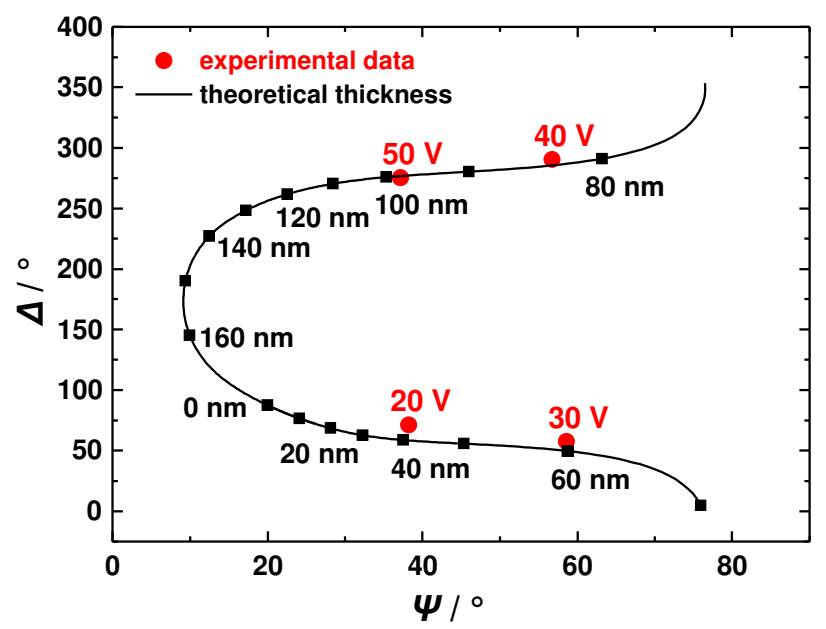

Figure 4.4. $\Delta / \Psi$ trajectory for the experimental data points (red) of the anodic $\mathrm{Ta}_{2} \mathrm{O}_{5}$ layers $\left(U_{\text {anod }}=20-50 \mathrm{~V}\right)$ and the scaled theoretical thickness (black curve with dots) of the ellipsometry model (Chapter 3.2.3, Table 3.6).

$\mathrm{Ta}_{2} \mathrm{O}_{5}$ layers were anodized at four different voltages (20, 30, 40 and $\left.50 \mathrm{~V}\right)$. The layer thickness plotted against the anodization voltage (Figure 4.5) presents a linear correlation $\left(\overline{\boldsymbol{R}}^{2}=0.998\right)^{\mathrm{XI}}$.

\footnotetext{
XI The adjusted coefficient of determination $\left(\overline{\boldsymbol{R}}^{2}\right)$ is used to describe the quality of a fit. It is calculated with the data analysis program Origin as follows: $\overline{\boldsymbol{R}}^{2}=\mathbf{1}-\frac{R S S / d f_{\text {error }}}{T S S / d f_{\text {total }}}$ with the residual sum of squares (RSS), the total sum of squares (TSS) and the degree of freedom (df).
} 
This indicates a linear increase of the $\mathrm{Ta}_{2} \mathrm{O}_{5}$ layer thickness with anodization voltage and supports the findings of earlier studies ${ }^{77}$ that the $\mathrm{Ta}_{2} \mathrm{O}_{5}$ layer grows linearly with the applied voltage. The slope of the linear fitting yields a layer growth coefficient of $(1.94 \pm 0.05) \mathrm{nm} / \mathrm{V}$. This value is comparable with the result reported by Kim et al. ${ }^{75}$ for a 22-nm-thick anodic $\mathrm{Ta}_{2} \mathrm{O}_{5}$ layer prepared in a citric acid solution at $10 \mathrm{~V}$.

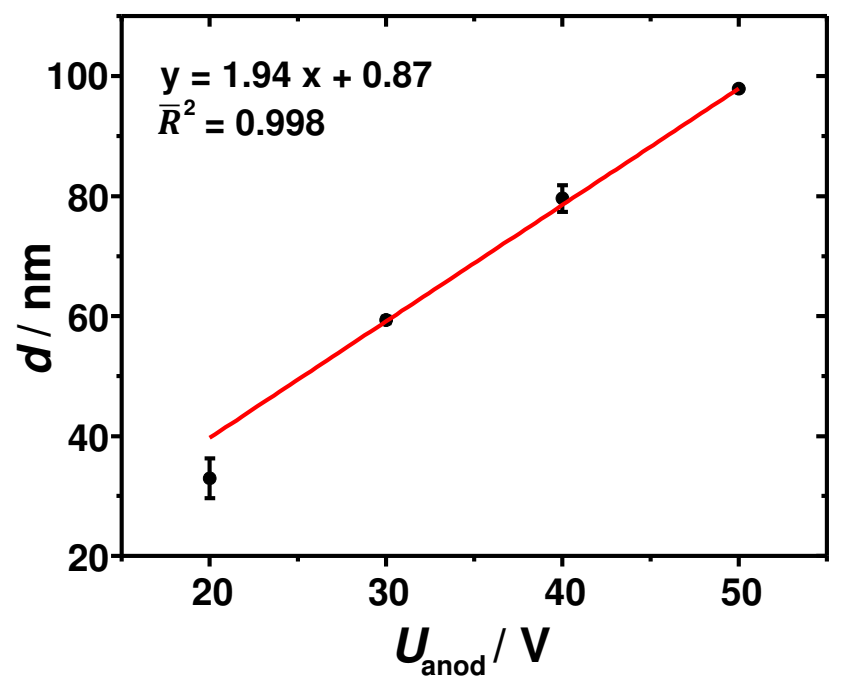

Figure 4.5. Thickness of $\mathrm{Ta}_{2} \mathrm{O}_{5}$ layers anodized at different voltages $(20-50 \mathrm{~V}, N \geq 3)$ and a linear fit (red).

In comparison with the $\mathrm{Ta}_{2} \mathrm{O}_{5}$ layers anodized at higher voltages $(30-50 \mathrm{~V})$, the $\mathrm{Ta}_{2} \mathrm{O}_{5}$ layer anodized at the lowest voltage $(20 \mathrm{~V})$ shows an outlier behavior concerning its thickness (Figure 4.5). On one hand, its mean value deviates most from the linear dependence of the layer thickness on the voltage. In comparison, the data points at the higher anodization voltages $(30-50 \mathrm{~V})$ exhibit this linear correlation very consistently. On the other hand, the layer thickness obtained at $20 \mathrm{~V}$ has a coefficient of variation ${ }^{\mathrm{XII}}(\mathrm{cv})^{143}$ of $10.2 \%$. This cv value is significantly higher than those determined for the other anodic $\mathrm{Ta}_{2} \mathrm{O}_{5}$ layers $(\mathrm{cv}<1 \%)$. These suggest that the $\mathrm{Ta}_{2} \mathrm{O}_{5}$ layers anodized at 30,40 and $50 \mathrm{~V}$ feature a significantly more predictable and more reproducible thickness than the oxide layer obtained at $20 \mathrm{~V}$.

XII Coefficient of variation (cv) is defined in the statistics as the ratio of the standard deviation to the mean in percentage ( $\mathrm{cv}=\frac{\sigma}{\bar{\mu}}$ ). It is used to compare the reproducibility for data sets with significantly different mean values. In this study, it was applied to evaluate the reproducibility of the thickness, the capacitance per unit area and the relative permittivity of the $\mathrm{Ta}_{2} \mathrm{O}_{5}$ layers. 


\subsubsection{3. $\mathrm{Ta}_{2} \mathrm{O}_{5}$ Layer Capacitance}

The electrical capacitance of tantalum oxide layers, either in the native form or fabricated through anodization, was investigated by means of PEIS (potentiostatic electrochemical impedance spectroscopy) in the citric acid solution (Chapter 3.2.1). The object under study here consists of the $\mathrm{Ta}_{2} \mathrm{O}_{5}$ layer and the citric acid solution.

The impedance spectra of the native tantalum oxide $\left(\boldsymbol{U}_{\text {anod }}=0 \mathrm{~V}\right)$ and the anodic oxide $\left(\boldsymbol{U}_{\text {anod }}=20-50 \mathrm{~V}\right)$ are shown in a Bode plot (Figure $\left.4.6 \mathrm{a}\right)$. The spectra reveal the frequencydependent responses of the impedance modulus, $|\boldsymbol{Z}|$, (dots) and the phase shift, $\emptyset$, (circles). Each impedance modulus curve, as well as each phase shift curve, shows two behaviors separated by a critical frequency (grey arrows demonstrated for a native tantalum oxide layer and for one anodized at $20 \mathrm{~V}$ ). At the critical frequency (Chapter 2.1.2.1), the impedance modulus shows a cutoff behavior; meanwhile, the phase shift has a value of $-45^{\circ}$. This is marked by the grey dashed lines. Above the critical frequency, the impedance modulus remains constant and independent from the frequency; meanwhile, the phase shift is between $0^{\circ}$ and $-45^{\circ}$. This behavior in the high frequency range reveals the resistance of the surrounding aqueous solution. This resistance is represented as $R_{1}$ in the circuit model (Figure $4.6 \mathrm{~b}$ ). Below the critical frequency, the impedance modulus features an inverse linear correlation to the frequency in the log-log graph; meanwhile, the phase shift is between $-45^{\circ}$ and $-90^{\circ}$. This impedance response indicates the capacitance of the object under study. This capacitance is represented as $C_{2}$ in the equivalent circuit model (Figure $4.6 \mathrm{~b}$ ).

(a)

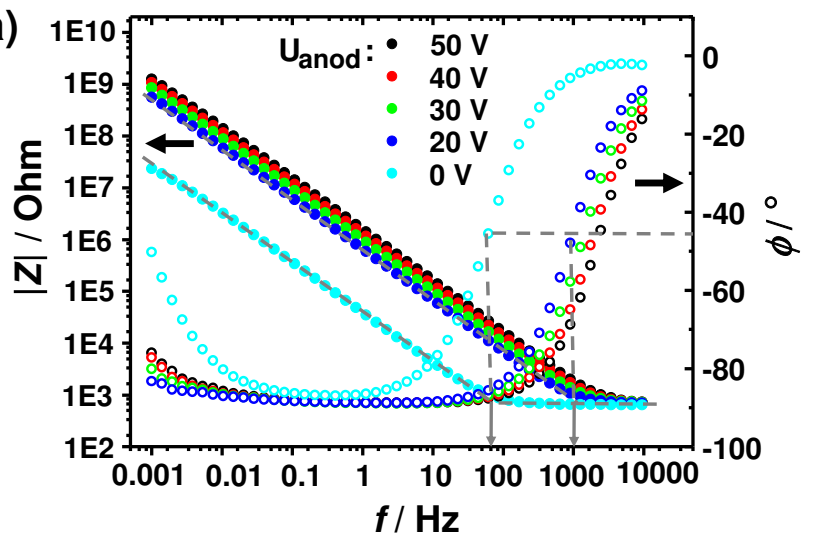

(b)

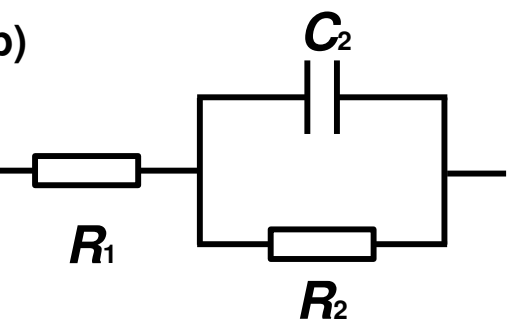

Figure 4.6. (a) Bode plot of impedance spectra with impedance modulus $(|Z|$, dots) and phase shift $\left(\varnothing\right.$, circles) against frequency for a native tantalum oxide layer $\left(U_{\text {anod }}=0 \mathrm{~V}\right)$ and for anodic $\mathrm{Ta}_{2} \mathrm{O}_{5}$ layers $\left(U_{\text {anod }}=20-50 \mathrm{~V}\right)$. Grey arrows: critical frequencies for a native oxide layer and for one anodized at $20 \mathrm{~V}$. (b) Equivalent circuit model for data fitting. $R_{1}$ : resistance of the surrounding solution, $C_{2}$ : capacitance of the object under study, $R_{2}$ : resistance of the $\mathrm{Ta}_{2} \mathrm{O}_{5}$ layer.

Moreover, a large drift of the phase shift toward $0^{\circ}$ was measured for the native tantalum oxide layer $\left(\boldsymbol{U}_{\text {anod }}=0 \mathrm{~V}\right)$ at very low frequencies below $12 \mathrm{mHz}$. In contrast, the anodized layers $\left(\boldsymbol{U}_{\text {anod }}=20-50 \mathrm{~V}\right)$ just show slight drifts of their phase shift in this low frequency region. This means a significant leakage current flowing through the native oxide layer. This finding reveals the native oxide layer to be unsuitable as the dielectric layer for the EWOD electrode. 
In comparison, the anodized oxide layers are superior in avoiding current leakage with a higher breakdown voltage. This result implies also the significantly higher resistance of the anodized oxide layers than that of the native one. This coincides with the finding that the anodized layers $(33.0-97.9 \mathrm{~nm}$, Figure 4.5$)$ are significantly thicker than the native one $(2-3 \mathrm{~nm})^{77,116}$ due to the linear correlation between the layer resistance $(\boldsymbol{R})$ and the thickness $(\boldsymbol{d})$ expressed in the equation $\boldsymbol{R}=\boldsymbol{\rho} \cdot \frac{\boldsymbol{d}}{\boldsymbol{\alpha}}$, with $\boldsymbol{\rho}$ standing for the resistivity and $\boldsymbol{\alpha}$ for the cross-section area ${ }^{48}$. The $\mathrm{Ta}_{2} \mathrm{O}_{5}$ layer resistance is represented in the equivalent circuit model as $R_{2}$ (Figure $4.6 \mathrm{~b}$ ).

By analyzing the impedance spectra (Figure 4.6 a) with the equivalent circuit model (Figure $4.6 \mathrm{~b}$ ), the capacitance of the object under study, $C_{2}$, was determined (Chapter 3.2.1). This capacitance may originate from the $\mathrm{Ta}_{2} \mathrm{O}_{5}$ layer $\left(\boldsymbol{C}_{\mathrm{Ta}_{2} \mathbf{O}_{5}}\right)$ and from the electrochemical double layer (EDL, $\left.\boldsymbol{C}_{\mathrm{dl}}\right)^{66,121}$, which is adjacent to the electrode surface in the solution. Due to their layer-on-layer structure, they can be modeled as two capacitors in series. The correlation between the $\boldsymbol{C}_{\mathrm{Ta}_{2} \mathbf{O}_{5}}, \boldsymbol{C}_{\mathbf{2}}$ and $\boldsymbol{C}_{\mathrm{dl}}$ can be expressed as below:

$$
\frac{1}{C_{\mathrm{Ta}_{2} \mathrm{O}_{5}}}=\frac{1}{C_{2}}-\frac{1}{C_{\mathrm{dl}}}
$$

To estimate the influence of the EDL capacitance, $\boldsymbol{C}_{\mathbf{2}}$ and $\boldsymbol{C}_{\mathrm{dl}}$ are compared based on their areal values. Since the counter electrode possesses a significantly larger contact area to the electrolyte than the working electrode, the $\boldsymbol{C}_{\mathrm{dl}}$ of the counter electrode can be considered negligible in comparison with the $\boldsymbol{C}_{\mathrm{dl}}$ of the working electrode. This means that the tantalum oxide layer and the EDL have an identical area. Thus, the areal value of $\boldsymbol{C}_{2}$ was determined with reference to the contact area $(\boldsymbol{A})$ of the working electrode to the electrolyte.

As a result, the $C_{2} / A$ yields $(103 \pm 8) \mathrm{nF} / \mathrm{mm}^{2}$ in case of the native tantalum oxide, while it ranges from $2.40 \mathrm{nF} / \mathrm{mm}^{2}$ to $5.88 \mathrm{nF} / \mathrm{mm}^{2}$ for the anodic $\mathrm{Ta}_{2} \mathrm{O}_{5}$ layers $\left(\boldsymbol{U}_{\text {anod }}=20-50 \mathrm{~V}\right)$. The typical $\boldsymbol{C} / \boldsymbol{A}$ value for a EDL lies in the range from $100 \mathrm{nF} / \mathrm{mm}^{2}$ to $500 \mathrm{nF} / \mathrm{mm}^{2}$ according to Orazem and Tribollet ${ }^{13}$ or further to $1000 \mathrm{nF} / \mathrm{mm}^{2}$ according to Butt et al. ${ }^{39,66}$.

Based on that, two cases are distinguished with the following conclusions:

Case 1: native tantalum oxide layer

The total capacitance per unit area $\left(\boldsymbol{C}_{2} / \boldsymbol{A}\right)$ and the contribution from the EDL are at the same magnitude or have a maximal difference by one magnitude. This result implies an essential influence of the EDL on the total capacitance for the object under study with a native tantalum oxide layer.

Case 2: anodic $\mathrm{Ta}_{2} \mathrm{O}_{5}$ layer

The total capacitance per unit area $\left(\boldsymbol{C}_{2} / \boldsymbol{A}\right)$ is significantly smaller than the contribution from the EDL by at least two magnitudes. According to Eq. 4.1, this result suggests that the influence of the EDL can be considered negligible. Therefore, the determined $\boldsymbol{C}_{2} / \boldsymbol{A}$ can be considered as the capacitance per unit area of the anodic $\mathrm{Ta}_{2} \mathrm{O}_{5}$ layer $\left(\boldsymbol{C}_{\mathrm{Ta}_{2} \mathbf{O}_{5}} / \boldsymbol{A}\right)$. Meanwhile, the 
capacitive component, $C_{2}$, in the equivalent circuit (Figure 4.6 b) represents the capacitance of the anodic $\mathrm{Ta}_{2} \mathrm{O}_{5}$ layers $\left(\boldsymbol{C}_{\mathrm{Ta}_{2} \mathbf{O}_{5}}\right)$.

To further characterize and compare the capacitive features of the anodic $\mathrm{Ta}_{2} \mathrm{O}_{5}$ layers fabricated at various voltages, the $\boldsymbol{C}_{\mathrm{Ta}_{2} \mathrm{O}_{5}} / \boldsymbol{A}$ values are plotted against the anodization voltage in Figure 4.7. The $\boldsymbol{C} / \boldsymbol{A}$ values are fitted with the function, $\mathrm{y}=\mathrm{a} / \mathrm{x}+\mathrm{b}$, to prove the following hypothesis: The capacitance per unit area should linearly correlate with the reciprocal voltage of anodization $\left(\frac{\boldsymbol{C}}{\boldsymbol{A}} \propto \frac{\boldsymbol{1}}{\boldsymbol{U}_{\text {anod }}}\right)$. This hypothesis is based on the finding, $\boldsymbol{d} \propto \boldsymbol{U}_{\text {anod }}$ (Figure 4.5, Chapter 4.1.2.2), and on the theoretical expression of the layer capacitance with the parallelplate capacitor model $\left(\frac{C}{A}=\frac{\varepsilon_{0} \varepsilon_{d}}{d}\right.$, Eq. 2.11).

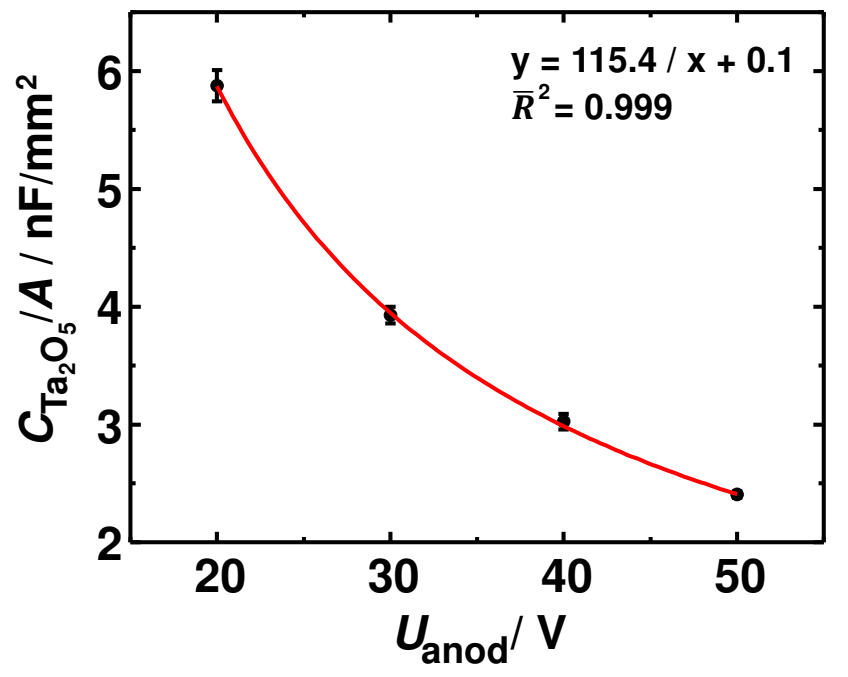

Figure 4.7. Capacitance per unit area of the anodic $\mathrm{Ta}_{2} \mathrm{O}_{5}$ layers $\left(U_{\text {anod }}=20-50 \mathrm{~V}, N \geq 3\right)$ fitted to the function, $y=a / x+b$, in red.

In Figure 4.7, the data set shows a trend of decreasing capacitance with increasing anodization voltage. Through the well-matched fitting $\left(\overline{\boldsymbol{R}}^{2}=0.999\right)$ with the function, $y=a / x+b$, this trend can be described more precisely: $\boldsymbol{C} / \boldsymbol{A}$ correlates with the reciprocal voltage. This result confirms the hypothesis suggested above. Furthermore, with respect to the result presented in Figure 4.5 ( $\boldsymbol{d} \propto \boldsymbol{U}_{\text {anod }}$ ), the outcome agrees well with the finding reported in earlier studies ${ }^{77}$, that the capacitance increases linearly with the reciprocal layer thickness.

A comparison among all data points shows that the highest value of the capacitance per unit area can be achieved through anodization at the lowest voltage $(20 \mathrm{~V})$. Unfortunately, this high capacitance is associated with a significantly higher coefficient of variation $(11.4 \%)$ in comparison with those obtained through anodization at 30,40 and $50 \mathrm{~V}$ (cv<2.5\%). This reveals the low reproducibility of the $\mathrm{Ta}_{2} \mathrm{O}_{5}$ layer anodized at $20 \mathrm{~V}$ with regard to its capacitance. 


\subsubsection{Relative Permittivity of $\mathrm{Ta}_{2} \mathrm{O}_{5}$ Layer}

Based on the two independently determined layer-properties, the thickness (Chapter 4.1.2.2) and the capacitance per unit area (Chapter 4.1.2.3), a material property of the anodic $\mathrm{Ta}_{2} \mathrm{O}_{5}$, the relative permittivity $\left(\boldsymbol{\varepsilon}_{\boldsymbol{d}}\right)$, can be calculated with the following equation:

$$
\varepsilon_{\mathrm{d}}=\frac{d}{\varepsilon_{0}} \cdot \frac{C}{A}
$$

This equation is obtained using Eq. 2.11 (Chapter 2.2.2.1) with $\varepsilon_{0}$ standing for the relative permittivity of vacuum. Since the thickness linearly correlates with the anodization voltage $\left(\boldsymbol{d} \propto \boldsymbol{U}_{\text {anod }}\right.$, Figure 4.5), while the capacitance per unit area depends linearly on the reciprocal voltage $\left(\frac{\boldsymbol{C}}{\boldsymbol{A}} \propto \frac{\mathbf{1}}{\boldsymbol{U}_{\mathrm{anod}}}\right.$, Figure 4.7$)$, the product of these two voltage-dependent parameters should be independent of the anodization voltage. Thus, $\boldsymbol{\varepsilon}_{\boldsymbol{d}}$ should also be voltageindependent according to Eq. 4.2.

To prove that the relative permittivity and the anodization voltage are independent of each other, the $\boldsymbol{\varepsilon}_{\boldsymbol{d}}$-value was determined for the $\mathrm{Ta}_{2} \mathrm{O}_{5}$ layers fabricated at $20,30,40$ and $50 \mathrm{~V}$ individually (Figure 4.8).

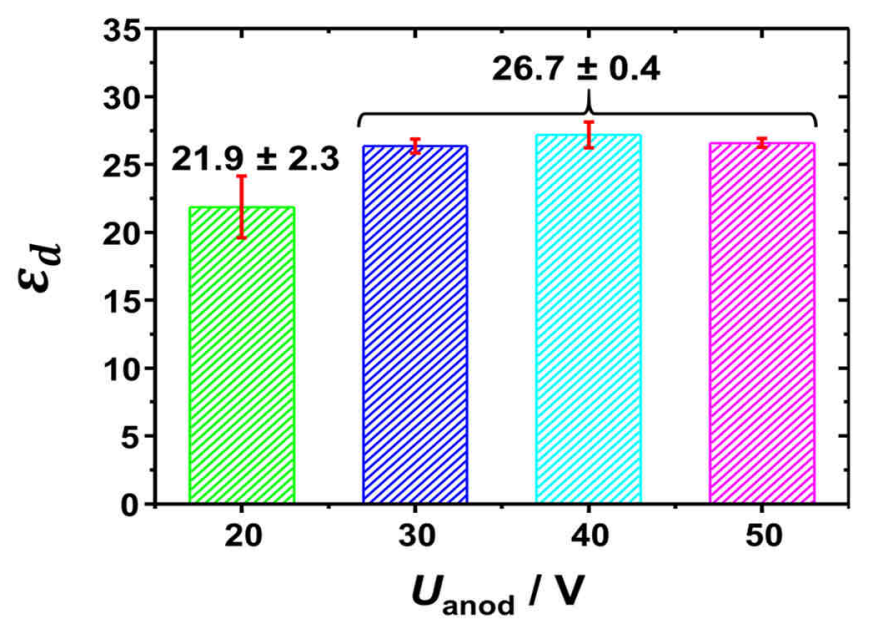

Figure 4.8. Relative permittivity of $\mathrm{Ta}_{2} \mathrm{O}_{5}$ layers anodized at different voltages $(20-50 \mathrm{~V}, N \geq 3)$.

The $\mathrm{Ta}_{2} \mathrm{O}_{5}$ layers anodized at the higher anodization voltages $(30,40$ and $50 \mathrm{~V}$ ) yield comparable values of the relative permittivity (Figure 4.8). Their mean value was determined to be 26.7 with a low standard deviation of 0.4 . This indicates that a constant, voltageindependent relative permittivity is available through anodization in this voltage region. Moreover, this relative permittivity value agrees very well with the literature values for the anodic $\mathrm{Ta}_{2} \mathrm{O}_{5}$ fabricated in citric acid solutions. A relative permittivity of 26.7 (anodization in $0.01 \%$ citric acid) was reported by Muth ${ }^{127}$ and $26.2-26.3$ (anodization in $0.5 \%$ citric acid) by Wilcox et al..$^{90}$. 
In contrast, the $\mathrm{Ta}_{2} \mathrm{O}_{5}$ layer anodized at $20 \mathrm{~V}$ shows a low relative permittivity of $21.9 \pm 2.3$. The percentage difference ${ }^{\mathrm{XIII}}$ between this low $\varepsilon_{\boldsymbol{d}}$-value and the high $\varepsilon_{\boldsymbol{d}}$-value from the other $\mathrm{Ta}_{2} \mathrm{O}_{5}$ layers $\left(\boldsymbol{U}_{\text {anod }}=30,40\right.$ and $\left.50 \mathrm{~V}\right)$ amounts to $19.8 \%$. This signalizes a significant negative variation of the relative permittivity in case of the anodization at $20 \mathrm{~V}$. Moreover, based on the linear correlation between the layer thickness and the anodization voltage (Figure 4.5), this result agrees with the finding reported by Kerrec et al:: a low $\varepsilon_{\boldsymbol{d}}$-value ( 18.5) for thin anodic $\mathrm{Ta}_{2} \mathrm{O}_{5}$ layers $(\leq 19 \mathrm{~nm})$ and a high $\boldsymbol{\varepsilon}_{\boldsymbol{d}}$-value $(\sim 27.5)$ for thick $\mathrm{Ta}_{2} \mathrm{O}_{5}$ layers ${ }^{74}$. As the reason for the $\varepsilon_{d}$-value difference, Kerrec et al. claimed that the relative permittivity in thin films is strongly influenced by the native tantalum oxide layer, which mainly contains $\mathrm{TaO}$ instead of $\mathrm{Ta}_{2} \mathrm{O}_{5}{ }^{74}$ This suggests that the relative permittivity of $\mathrm{TaO}$ is lower than that of $\mathrm{Ta}_{2} \mathrm{O}_{5}$. Thus, the thin layer below a certain thickness threshold possesses a low $\boldsymbol{\varepsilon}_{\boldsymbol{d}}$-value due to the low percentage ratio of $\mathrm{Ta}_{2} \mathrm{O}_{5}$ to $\mathrm{TaO}$ in the layer.

Furthermore, the $\varepsilon_{\boldsymbol{d}}$-value of the $\mathrm{Ta}_{2} \mathrm{O}_{5}$ layer anodized at $20 \mathrm{~V}$ has a cv of $10.4 \%$. This $\mathrm{cv}$ is significantly higher than those for the other oxide layers (cv $\leq 3.5 \%$ ). The error in the relative permittivity arises from the error in the layer thickness ( $\mathrm{cv}=10.2 \%)$ and that in the capacitance per unit area ( $\mathrm{cv}=11.4 \%$ ). The high cv-values imply the low reproducibility of the $\mathrm{Ta}_{2} \mathrm{O}_{5}$ layer fabricated through anodization at $20 \mathrm{~V}$. Since these three parameters are key to the EWOD effect (Chapter 4.1.1), their high uncertainty in this case makes this $\mathrm{Ta}_{2} \mathrm{O}_{5}$ layer inadequate as the dielectric layer in an EWOD electrode.

To achieve the low-voltage $\mathrm{EWOD}$, the $\mathrm{Ta}_{2} \mathrm{O}_{5}$ layer anodized at $30 \mathrm{~V}$ was considered to be the most appropriate dielectric layer in the EWOD electrode. This $\mathrm{Ta}_{2} \mathrm{O}_{5}$ layer possesses the lowest layer thickness $(59.4 \pm 0.6 \mathrm{~nm}$, Figure 4.5$)$ and the highest capacitance per unit area $\left(3.93 \pm 0.07 \mathrm{nF} / \mathrm{mm}^{2}\right.$, Figure 4.7) among the $\mathrm{Ta}_{2} \mathrm{O}_{5}$ layers fabricated at higher voltages $\left(\boldsymbol{U}_{\text {anod }}=30-50 \mathrm{~V}\right)$ in this study. These two layer-properties and its high relative permittivity $(26.3 \pm 0.5)$ benefit the EWOD effect to occur at a low voltage according to the YoungLippmann equation (Eq. 2.26). Moreover, its low cv-values for the three key parameters, $\boldsymbol{d}(0.9 \%), \boldsymbol{C} / \boldsymbol{A}(1.8 \%)$ and $\boldsymbol{\varepsilon}_{\boldsymbol{d}}(2.0 \%)$, confirm the high reproducibility of the dielectric properties and thus assure the reliability of its high dielectric quality. Therefore, this $\mathrm{Ta}_{2} \mathrm{O}_{5}$ layer $\left(\boldsymbol{U}_{\text {anod }}=30 \mathrm{~V}\right)$ was preferred as the dielectric initial layer for the LV-EWOD electrodes used in the further investigations into the EWOD effect.

XIII Percentage difference is the difference between two values with reference to their average value in percentage. It is mathematically expressed as: $\frac{\left|x_{1}-x_{2}\right|}{\left(x_{1}+x_{2}\right) / 2} \cdot 100 \%$. 


\subsubsection{ODTS Monolayer with Hydrophobic Smooth Surface}

On top of the $\mathrm{Ta}_{2} \mathrm{O}_{5}$ initial layer, a silane layer, specifically a thin film of ODTS (Octadecyltrichlorosilane), covers the EWOD electrode (Figure 4.1) to render its surface hydrophobic. The ODTS layer was fabricated through the wet-chemical silanization (Chapter 3.1.4) based on the self-assembling principle ${ }^{39,102 .}$

\subsubsection{Topology and Hydrophobicity of ODTS Surface}

A representative ODTS coating on the anodic $\mathrm{Ta}_{2} \mathrm{O}_{5}$ layer $\left(\boldsymbol{U}_{\text {anod }}=30 \mathrm{~V}\right)$ shows a homogeneous smooth surface with a few round particles in different nanometer sizes in its AFM image (Figure 4.9). The surface roughness has an increased root-mean-square value of $1.45 \mathrm{~nm}$ in comparison to that of the $\mathrm{Ta}_{2} \mathrm{O}_{5}$ layer $(0.31 \mathrm{~nm}$, Figure $4.2 \mathrm{a})$.

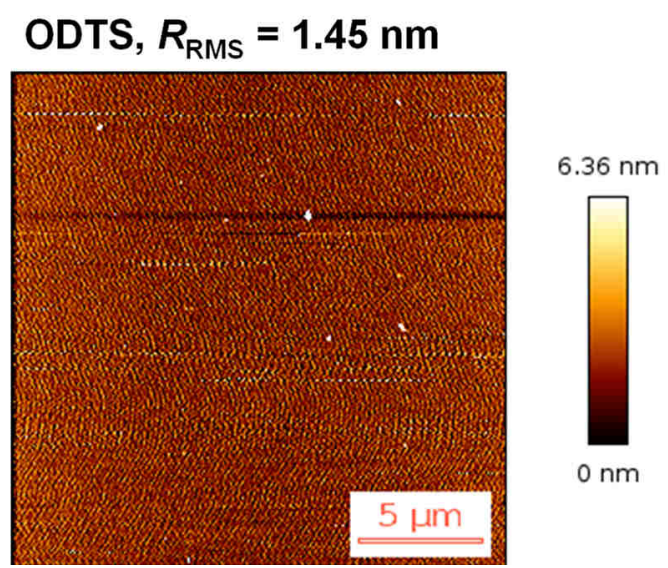

Figure 4.9. AFM image besides the color-height correlation for an ODTS coating with a rootmean-square roughness of $1.45 \mathrm{~nm}$ on a $\mathrm{Ta}_{2} \mathrm{O}_{5}$ initial layer $\left(U_{\text {anod }}=30 \mathrm{~V}\right)$.

The increased surface roughness can mainly result from the particles, which can be the oligomers of ODTS formed through a water-induced aggregation. Despite the preventive dry operation condition, water in the ambient air may contaminate ODTS due to the gaps in the preparation and the coating process chain. Chen et al. ${ }^{144}$ also reported particles on the ODTS monolayer generated through vapor deposition on $\mathrm{SiO}_{2}$. Due to the novel combination of ODTS and $\mathrm{Ta}_{2} \mathrm{O}_{5}$, no other report about this material combination was available for the literature values for comparison.

Besides the determination of the topological roughness, the ODTS coating was investigated for its surface hydrophobicity through measurements of the contact angle. Its contact angle hysteresis was determined to quantify the resistance against droplet motion on its surface, since this resistance can be considered as a static friction ${ }^{37}$.

In Table 4.2, the ODTS coating presents a large water contact angle of $104.7^{\circ} \pm 1.0^{\circ}$, while the $\mathrm{Ta}_{2} \mathrm{O}_{5}$ initial layer shows a very small contact angle (Chapter 4.1.2.1). This indicates that the fourth fabrication step, silanization with ODTS (Chapter 3.1.4), successfully converted the electrode surface from very hydrophilic to hydrophobic. Moreover, the electrode surface of ODTS on the $\mathrm{Ta}_{2} \mathrm{O}_{5}$ exhibits a contact angle hysteresis of $11.6^{\circ} \pm 0.6^{\circ}$. 
Table 4.2. Contact angle and contact angle hysteresis determined for the anodic $\mathrm{Ta}_{2} \mathrm{O}_{5}$ surface $\left(U_{\text {anod }}=20-50 \mathrm{~V}\right)$ and for the ODTS surface based on $\mathrm{a} \mathrm{Ta}_{2} \mathrm{O}_{5}$ initial layer.

\begin{tabular}{ccc}
\hline interfacial circumstance & $\mathrm{Ta}_{2} \mathrm{O}_{5} /$ water / air & ODTS / water / air \\
\hline static contact angle $\left(\boldsymbol{\theta}_{\mathbf{0}}\right)\left[^{\circ}\right]$ & $11.2 \pm 3.3$ & $104.7 \pm 1.0$ \\
contact angle hysteresis $(\Delta \boldsymbol{\theta})\left[^{\circ}\right]$ & - & $11.6 \pm 0.6$ \\
\hline
\end{tabular}

The ODTS surface generated in this study features a comparable contact angle and a slightly lower contact angle hysteresis in comparison to another alkyl silane, (n-decyl)trichlorosilane (DTS), coated on a wet-cleaned $\mathrm{Ta}_{2} \mathrm{O}_{5}$ layer $\left(\boldsymbol{\theta}_{\mathbf{0}}=108.4^{\circ}, \Delta \boldsymbol{\theta}=13.8^{\circ}\right)^{116}$.

A comparison with the ODTS-coatings on other substrates reveals slight discrepancy in the contact angle and contact angle hysteresis. A larger contact angle was reported for the ODTS layer deposited on $\mathrm{SiO}_{2}\left(\boldsymbol{\theta}_{\mathbf{0}}=112^{\circ}\right)^{97}$. The ODTS layer on silicon nitride oxide was reported to have lower values in both contact angle and contact angle hysteresis $\left(\boldsymbol{\theta}_{\mathbf{0}}=101^{\circ}, \Delta \boldsymbol{\theta}=6^{\circ}\right)^{73}$.

This comparison indicates that ODTS layers deposited on initial layers consisting of various materials can present differences in their surface hydrophobicity and in the friction against droplet motion on their surfaces. This interfacial friction or resistance may result from the surface roughness and from the surface topology on the macroscopic and microscopic level as elucidated below.

First, the roughness of the initial layer strongly influences the roughness of the top layer due to the very thin monolayer of ODTS in the nanometric scale (Chapter 4.1.3.2). In comparison to the typically used substrates for silane coatings, such as silicon, silicon dioxide $\left(\boldsymbol{R}_{\mathbf{R M S}}=0.46 \AA\right)^{97}$ and silicon nitride oxides, the anodic $\mathrm{Ta}_{2} \mathrm{O}_{5}$ (Figure 4.2) has a significantly rougher surface. This roughness of the metal oxides differs probably due to their fabrication process and their material properties, such as the lattice structure and the correlated distance between the possible binding sites for silane molecules. Thus, the lattice structure of substrates essentially affects the molecular arrangement of a self-assembled silane monolayer. The resulted different package density and the tilt angle of the silane molecules on different metal oxide initial layer can influence the microscopic surface roughness.

Second, this roughness may originate from the inhomogeneity of the self-assembled ODTS monolayer on a molecular level as reported in a publication ${ }^{145}$ within this doctoral research. During self-assembly, the head groups of ODTS molecules bond with $\mathrm{Ta}_{2} \mathrm{O}_{5}$ via silanol-groups and become immobilized on the surface. Besides this covalent bonding, the ODTS molecules non-covalently bind with each other through the van der Waals interaction between their hydrophobic alkyl chains. The long alkyl chains in a monolayer may have different conformations. Since the identical conformation is essential for molecules to be regularly ordered, slight difference in the conformation may result in irregular orientation and nonuniform arrangement of the molecules. 
This microscopic inhomogeneity may originate from impurities, such as ODTS-isomers and -oligomers, and result in a loose packed layer and local discrepancy in the surface topology (roughness). The ODTS surface roughness may affect the interfacial friction against the droplet movement during electrowetting and thus influence the dynamics of the wetting and dewetting process.

Furthermore, the high hydrophobicity of the ODTS surface could induce a strong repulsive force against the hydrophilic cantilever made of $\mathrm{Si}_{3} \mathrm{~N}_{4}{ }^{132}$ and result in artefacts in AFM-imaging. Therefore, the ODTS surface was scanned under the qualitative imaging mode in AFM by applying a constant force to the cantilever, to avoid possible errors by using the tapping mode.

In addition, to achieve the hydrophobic electrode surface, ODTS and a fluorinated silane, (tridecafluoro-1,1,2,2-tetrahydrooctyl) trichlorosilane, were applied using diverse coating methods, including wet-chemical deposition in various solvents and vapor deposition. The fluorinated silane coatings generated through each deposition method contained significantly more aggregates in irregular forms and sizes (AFM images not shown here) in comparison to the ODTS coatings. This may be caused by the very high sensitivity of the fluorinated silane molecules to moisture. Due to the low quality and low reproducibility, these coatings are inadequate for usage in the EWOD electrode. In this study, the ODTS coating obtained through the wet deposition in toluene was found to be the most promising candidate as the top layer in the LV-EWOD electrode. McGovern et al. reported also about the well-packed SAM of silane on glass through silanization in toluene ${ }^{146}$. The high quality of this ODTS coating is based on its superior reproducibility and high homogeneity with reference to the AFM images and the contact angle results. In consequence, merely this ODTS coating was thoroughly investigated with respect to the EWOD-relevant parameters.

\subsubsection{ODTS as Monolayer}

The ODTS thin layer deposited on the anodic $\mathrm{Ta}_{2} \mathrm{O}_{5}$ surface is transparent. Its thickness was determined with the ellipsometry to be $(2.3 \pm 0.2) \mathrm{nm}$.

The length of an ODTS molecule along its alkyl chain was estimated to be $2.62 \mathrm{~nm}$. This was calculated based on the monolayer model postulated by Wassermann et al. ${ }^{147}$ for methylterminated siloxane deposited on $\mathrm{SiO}_{2}$. In that model, the silane molecules in the SAM (selfassembled monolayer) are oriented nearly perpendicularly to the $\mathrm{SiO}_{2}$ surface with a tilt angle of $10^{\circ}$ from the vertical axis. The monolayer thickness $(t)$ is expressed as $t_{\mathbf{m}}=1.26 \boldsymbol{m}+4.78$ (in $\AA$ ) with $\boldsymbol{m}$ standing for the number of $\mathrm{CH}_{2}$-units in the alkyl chain. Moreover, this estimated molecule length agrees with the ellipsometirc thickness of ODTS monolayer on $\mathrm{Si} / \mathrm{SiO}_{2}$ reported by Wang et al. $(2.4 \mathrm{~nm} \pm 0.2 \mathrm{~nm}, 2.6 \mathrm{~nm} \pm 0.2 \mathrm{~nm})^{97}$ and by Bush et al. $(2.7 \mathrm{~nm} \pm 0.1 \mathrm{~nm})^{148}$. 
Furthermore, SAM of silane on $\mathrm{Ta}_{2} \mathrm{O}_{5}$ surface was studied by De Palma et al. ${ }^{116}$ with (n-decyl)trichlorosilane (DTS). In comparison to ODTS $(\boldsymbol{m}=17)$, DTS has the analog structure but a shorter alkyl chain with $9 \mathrm{CH}_{2}$-units. A thickness of $(1.11 \pm 0.01) \mathrm{nm}$ was reported besides a tilt angle of $45^{\circ}$ between the alkyl chain and the $\mathrm{Ta}_{2} \mathrm{O}_{5}$ surface for DTS. Based on their study and the length of a C-C single bond $(0.153-0.154 \mathrm{~nm})^{149-150}$, a theoretical monolayer thickness of ODTS on the $\mathrm{Ta}_{2} \mathrm{O}_{5}$ surface was estimated at $2.0 \mathrm{~nm}$.

In this study, the determined thickness value $(2.3 \mathrm{~nm} \pm 0.2 \mathrm{~nm})$ of the ODTS layer is lower than the estimated value of the ODTS molecule length $(2.6 \mathrm{~nm})$. This difference indicates the fabricated ODTS coating to be a monolayer with the ODTS molecules tilting on the $\mathrm{Ta}_{2} \mathrm{O}_{5}$ surface. In addition, the determined thickness is higher than its theoretical thickness $(2.0 \mathrm{~nm})$ derived from that of the DTS monolayer. This can result from the particles on the ODTS surface, which also contributed to the average layer thickness over the entire region of interest (ROI) in the ellipsometry measurement.

\subsubsection{Capacitance of $\mathrm{Ta}_{2} \mathrm{O}_{5}-O D T S-B i l a y e r$ and of ODTS Layer}

The capacitance per unit area of the ODTS- $\mathrm{Ta}_{2} \mathrm{O}_{5}$-bilayer was characterized by means of EIS using single sinusoidal signals and imaging. In this study, measurements were conducted with (i) an in-house programmed EIS associated with imaging for integration with EWOD (Chapter 3.3.1, 3.3.2) and (ii) a commercial potentiostat (Chapter 3.2.1) for calibration. Based on the $\boldsymbol{C} / \boldsymbol{A}$ value of the ODTS-Ta $\mathrm{O}_{5}$-bilayer and that of the $\mathrm{Ta}_{2} \mathrm{O}_{5}$-layer (Chapter 4.1.2.3), the capacitance per unit area was determined for the ODTS layer.

First, the capacitance per unit area $(\boldsymbol{C} / \boldsymbol{A})$ was determined for the ODTS-Ta $\mathrm{O}_{5}$-bilayer with the in-house programed EIS in a two-electrode configuration (Figure 3.13, Chapter 3.3.1). The object under investigation was a $3 \mu \mathrm{L}$ droplet of $200 \mathrm{mM} \mathrm{KCl}$ resting on the LW-EWOD electrode. The applied sinusoidal signal had a voltage amplitude of $0.5 \mathrm{~V}$ and an offset of $0.5 \mathrm{~V}$. The involved area of the dielectric bilayer was measured as the contact area $(\boldsymbol{A})$ between the droplet and the EWOD electrode. It was acquired through the simultaneous imaging for EIS measurement at each frequency. The $\boldsymbol{C} / \boldsymbol{A}$-value was calculated using the capacitance $(\boldsymbol{C})$ determined through EIS and the droplet contact area on the EWOD electrode determined by analysis of optical images of the droplet (Chapter 3.3.1, 3.3.2). The $\boldsymbol{C} / \boldsymbol{A}$ was plotted against frequency in the region from $100 \mathrm{~Hz}$ to $10 \mathrm{kHz}$, as shown in Figure 4.10.

Within the frequency measuring range, $\boldsymbol{C} / \boldsymbol{A}$ generally remains constant. This yields a mean value of $(2.66 \pm 0.16) \mathrm{nF} / \mathrm{mm}^{2}$. This result was verified by PEIS-measurement using a commercial potentiostat and the separated imaging system under the same measuring conditions $\left(2.65 \mathrm{nF} / \mathrm{mm}^{2} \pm 0.04 \mathrm{nF} / \mathrm{mm}^{2}, \boldsymbol{N}=3\right)$. The well comparable mean values reveal the high accuracy of the in-house programmed EIS for integration with EWOD. 


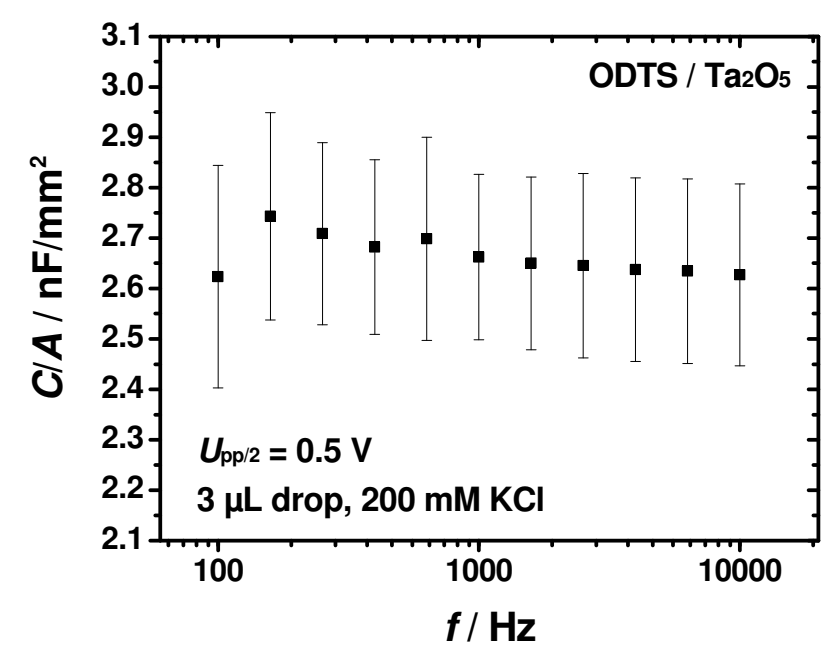

Figure 4.10. Capacitance per unit area for the ODTS-Ta $\mathrm{O}_{5}$-bilayer determined with EIS using a single sinusoidal signal $\left(U_{\mathrm{pp} / 2}=0.5 \mathrm{~V}\right)$ on a $3 \mu \mathrm{L}$ drop of $200 \mathrm{mM} \mathrm{KCl}$ resting on the LV-EWOD electrode $(N=3)$.

This determined capacitance per unit area can be considered as the ODTS- $\mathrm{Ta}_{2} \mathrm{O}_{5}$-bilayer capacitance. This claim is based on the significantly smaller capacitance of the ODTS- $\mathrm{Ta}_{2} \mathrm{O}_{5}-$ bilayer than that of the EDL on the droplet - platinum wire - interface by two magnitudes. Despite its area being larger by one order of magnitude, the dielectric bilayer has the significantly larger thickness by three orders of magnitude and lower relative permittivity than the EDL. Therefore, the dielectric capacitance dominates in the system under investigation and the EDL capacitance can be considered negligible.

Moreover, the plot reveals a slight consistent drift of $\boldsymbol{C} \boldsymbol{A}$. For this observation, two reasons may be responsible: On the one hand, the recorded droplet - EWOD electrode - contact area $(\boldsymbol{A})$ declines with the proceeding of the measurement from the highest to the lowest frequency. This means that the droplet shrinks slightly due to evaporation with the frequency decrease. On the other hand, the recorded capacitance increased slightly with the frequency decrease. This could result from the prolonged period $\left(T=\frac{1}{f}\right)$ for the storage of the electrical potential energy, which causes a higher apparent capacitance.

Second, the ODTS-Ta ${ }_{2} \mathrm{O}_{5}$-bilayer $\left(\boldsymbol{C} / \boldsymbol{A}=(2.66 \pm 0.16) \mathrm{nF} / \mathrm{mm}^{2}\right.$, Figure 4.10) and the anodic $\mathrm{Ta}_{2} \mathrm{O}_{5}$ layer $\left(\boldsymbol{U}_{\text {anod }}=30 \mathrm{~V}, \boldsymbol{C} / \boldsymbol{A}=(3.93 \pm 0.07) \mathrm{nF} / \mathrm{mm}^{2}\right.$, Figure 4.7) exhibited capacitive characteristics. This indicates the ODTS layer to also behave as a capacitor in the bilayer stack. Based on the layer-by-layer structure, this supports the validity of the equivalent circuit model (Figure 2.12 b, Chapter 2.2.2.1) to represent the $\mathrm{Ta}_{2} \mathrm{O}_{5}$ layer and the ODTS layer as two capacitors in a series connection. Thus, the capacitance per unit area $(\boldsymbol{C} / \boldsymbol{A})$ value for the ODTS layer was calculated according to the following correlation:

$$
\frac{A}{C_{\mathrm{ODTS}}}=\frac{A}{C_{\text {bilayer }}}-\frac{A}{C_{\mathrm{Ta}_{2} \mathrm{O}_{5}}}
$$

This yields a $\boldsymbol{C} / \boldsymbol{A}$-value of $(8.23 \pm 1.56) \mathrm{nF} / \mathrm{mm}^{2}$ for the ODTS layer. The uncertainty was calculated according to Gaussian error propagation. 


\subsubsection{Relative Permittivity of ODTS Monolayer}

Based on the plate capacitor model, the relative permittivity was determined for the ODTS monolayer with its thickness $(2.3 \mathrm{~nm} \pm 0.2 \mathrm{~nm}$, Chapter 4.1.3.2) and its capacitance per unit area $\left(8.23 \mathrm{nF} / \mathrm{mm}^{2} \pm 1.56 \mathrm{nF} / \mathrm{mm}^{2}\right.$, Chapter 4.1.3.3). In analogy to the case of the anodic $\mathrm{Ta}_{2} \mathrm{O}_{5}$ layer (Chapter 4.1.2.4), the $\varepsilon_{\boldsymbol{d}}$-value was calculated for the ODTS monolayer with Eq. 4.2 to be $2.1 \pm 0.4$.

This $\varepsilon_{\boldsymbol{d}}$-value is close to the medium $\boldsymbol{\varepsilon}_{\boldsymbol{d}}$-value suggested by Gala and Zollo ${ }^{79}$ in a simulation study about the relative permittivity of the ODTS monolayer. The authors postulated the $\boldsymbol{\varepsilon}_{\boldsymbol{d}^{-}}$ value for an ODTS monolayer on a silicon substrate in the range from 1.6 to 3.1 depending on the coverage ratio.

According to Gala and Zollo ${ }^{79}$, the medium $\boldsymbol{\varepsilon}_{\boldsymbol{d}}$-value may be induced by a medium package density of the fabricated ODTS layer on the $\mathrm{Ta}_{2} \mathrm{O}_{5}$ surface and could be limited by the Ta-O$\mathrm{Si}$ adhesion group between the ODTS surface and the $\mathrm{Ta}_{2} \mathrm{O}_{5}$ surface.

The medium package density may originate from the material property of $\mathrm{Ta}_{2} \mathrm{O}_{5}$ as the immobilization substrate and from the layer property of the self-assembled ODTS monolayer. First, in comparison to silicon, $\mathrm{Ta}_{2} \mathrm{O}_{5}$ possesses a larger lattice distance. This means a larger distance between the accessible binding sites for the ODTS molecules on the $\mathrm{Ta}_{2} \mathrm{O}_{5}$-surface . Additionally, De Palma et al. ${ }^{116}$ suggested that not every binding site on the $\mathrm{Ta}_{2} \mathrm{O}_{5}$-surface is linked to a silane molecule. These factors may lead to the large distance between the neighboring adhesion groups and thus a large tilting angle of the alkyl chains in the ODTS molecules. In this study, the lower value of the determined layer thickness than the estimated ODTS molecule length (Chapter 4.1.3.2) confirms the molecule tilting and the limited package density. Second, the self-assembled ODTS monolayer may contain local inhomogeneity. The inhomogeneous arrangement of the ODTS molecules could be caused by different configurations of the alkyl chains regarding their torsion and orientation. This is suggested for the slight surface roughness in the topology analysis with AFM (Chapter 4.1.3.1) and could reduce the packing density of the alkyl chains.

The packing density of the adhesion groups and the alkyl chains influences their partial dipole contribution to the entire polarizability of the ODTS layer and hence influences the relative permittivity. 


\subsubsection{Multilayer Dielectric Stack Including an Oil Layer}

Besides the solid ODTS- $\mathrm{Ta}_{2} \mathrm{O}_{5}$-bilayer of the LV-EWOD-electrode, an oil layer of tetradecane contributes a fluid dielectric layer to the MDS model as shown in Figure 4.1. This oil layer is entrapped between the droplet and the hydrophobic EWOD electrode due to the application of an oil environment in the EWOD setup. In comparison with air, an oil surrounding immiscible to the droplet should benefit the low-voltage EWOD in diverse aspects: increasing the contact angle of the droplet in its resting state, reducing the surface friction against the droplet motion and preventing droplet evaporation. In addition, the most applications of droplet-based microfluidics use aqueous droplets in an oil as carrier medium ${ }^{151}$.

\subsubsection{Low Surface Friction in Oil Surrounding}

The advantages of the oil environment against the air ambience were characterized by the results of contact angle and contact angle hysteresis shown in Table 4.3. These two parameters were determined with a water droplet in air on the LV-EWOD electrode before its oil conditioning (Chapter 3.1.5) and in tetradecane (oil) respectively (Chapter 3.2.5-3.2.6).

Table 4.3. Contact angle and contact angle hysteresis of a $3 \mu \mathrm{L}$ water droplet on the LV-EWOD electrode in air and in tetradecane.

\begin{tabular}{ccc}
\hline interfacial circumstance & ODTS / water/ air & ODTS / water / tetradecane \\
\hline static contact angle $\left(\boldsymbol{\theta}_{\mathbf{0}}\right)\left[^{\circ}\right]$ & $104.7 \pm 1.0$ & $160.7 \pm 0.8$ \\
contact angle hysteresis $(\Delta \boldsymbol{\theta})\left[^{\circ}\right]$ & $11.6 \pm 0.6$ & $0.9 \pm 0.4$ \\
\hline
\end{tabular}

In tetradecane, the water droplet presents a large contact angle of $160.7^{\circ}$. This contact angle is significantly larger than that in air by $56^{\circ}$. Moreover, a water droplet in tetradecane exhibits a very low contact angle hysteresis of $0.9^{\circ}$. This value is significantly smaller than that in air by $10.7^{\circ}$ (Table 4.3 ).

The large contact angle and the very low contact angle hysteresis are two essential and advantageous features for achieving the EWOD effect at low voltages, as reasoned below.

First, the static contact angle corresponds to the droplet contact angle without applied voltage $\left(\boldsymbol{\theta}_{\mathbf{0}}\right)$ in the Young-Lippmann equation (Eq. 2.2). The large $\boldsymbol{\theta}_{\mathbf{0}}$ value is a key approach to the low-voltage electrowetting, as elucidated at the beginning of Chapter 4.1.

Second, the very low contact angle hysteresis measured in tetradecane by tilting method (Chapter 3.2.6) means the droplet requires just a slight difference between the advancing and the receding contact angle for its motion. This result suggests a very low surface friction against droplet motion. Transferred into the EWOD circumstance, the easy motion may lead to the rapid deformation of the droplet concerning its simultaneous alteration of the contact area and 
the contact angle. This implies good dynamic responses in the voltage-induced wetting process and more importantly in the passive dewetting process. Moreover, the simultaneous alteration of the contact area and the contact angle would be an essential condition for the replacement of the optical detection through area-related electrical measurements in the future research towards compact and low-cost EWOD-EIS devices.

Therefore, the application of an oil environment instead of the air ambience enhances the efficiency of the low-voltage EWOD effect. The enlarged contact angle and the reduced contact angle hysteresis of a water droplet on the solid surface of ODTS indicate the interfacial interaction between the aqueous droplet and the electrode surface to be strongly reduced by the oil surrounding. With a view of the Young's equation (Eq. 2.1), the larger contact angle in the oil than that in air can be attributed to the lower interfacial tensions on the oil-solid-interface and on the water-oil-interface than those corresponding surface tensions in air. With a microscopic view, the tetradecane (oil) molecules and the ODTS molecules may be weakly bound through the van der Waals interaction between their long alkane chains. Based on that, the immersion of the LV-EWOD electrode in the tetradecane for sufficient time may enable the silanized surface to be saturated with oil. This may result in a thin oil layer existing between the droplet and the substrate. This oil layer may act as lubricant and thus reduce or avoid the droplet pinning on the surface as postulated in the literature ${ }^{50}$.

\subsubsection{Capacitance of MDS and of Oil Layer}

Concerning the equivalent circuit model for the MDS (Figure $2.12 \mathrm{~b}$, Chapter 2.2.2.1), the oil top layer, the ODTS middle layer and the $\mathrm{Ta}_{2} \mathrm{O}_{5}$ initial layer $\left(\boldsymbol{U}_{\text {anod }}=30 \mathrm{~V}\right)$ are theoretically described as three plate capacitors in series. Based on this, the oil-ODTS- $\mathrm{Ta}_{2} \mathrm{O}_{5}$-stack (MDS) consisting of one fluid and two solid dielectric layers should feature a capacitor behavior. This hypothesis was proven by a CV measurement (Chapter 3.2.2) to characterize the capacitive behavior through a charging and discharging process. Moreover, analog to the $\boldsymbol{C} / \boldsymbol{A}$ determination of the ODTS- $\mathrm{Ta}_{2} \mathrm{O}_{5}$-bilayer (Chapter 4.1.3.3), the MDS was characterized for its capacitance per unit area with the in-house programmed EIS for the integration with EWOD (Chapter 3.3.2). The $\boldsymbol{C} / \boldsymbol{A}$ value of the oil layer was determined based on that of the MDS and that of the ODTS-Ta $\mathrm{O}_{5}$-bilayer (Figure 4.10).

First, to characterize the capacitive behavior of the oil-ODTS- $\mathrm{Ta}_{2} \mathrm{O}_{5}$-stack, CV was measured on an aqueous $200 \mathrm{mM} \mathrm{KCl}$ droplet resting in oil on the LV-EWOD electrode (Chapter 3.2.2). The cyclic voltammogram (Figure 4.11) shows the averaged current from three cycles against the applied voltage between the LV-EWOD electrode and the $\mathrm{Ag} / \mathrm{AgCl}$ reference electrode. 


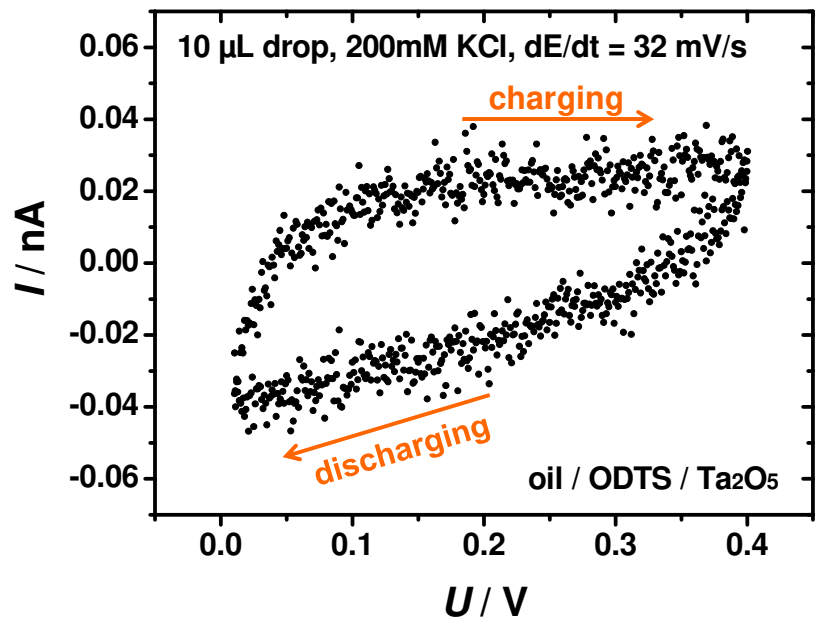

Figure 4.11. Cyclic voltammogram of the MDS during the charging and the discharging process (progression shown by orange arrows). Measurement at a scan rate of $32 \mathrm{mV} / \mathrm{s}$ with a $10 \mu \mathrm{L}$ drop of $200 \mathrm{mM} \mathrm{KCl}$ resting in oil on the LV-EWOD electrode (averaged data of 3 cycles).

The cyclic voltammogram shows an asymmetry of the current progression in the charging process and in the discharging process.

In the charging process with the voltage ramp from $0.0 \mathrm{~V}$ to $0.4 \mathrm{~V}$, the current starts with a sharp rise, gradually reaches its maximal value and remains almost constant in a saturated state. This rapidly reached constant current indicates the presence of a pure displacement current. It corresponds to the displacement of charge carriers within a capacitor upon penetration by an electric field. This characteristic displacement current reflects a charging process of a nearly ideal capacitor.

In the discharging process with the reversed ramp back to $0.0 \mathrm{~V}$, the current shows first a steep decline until $0 \mathrm{~A}$. It is followed by a further decrease in the negative region with a nearly constant slope. The latter consistent decline indicates that a faradaic current flows besides a displacement current. The faradaic current is very low, since the total recorded current locates in the sub-nA region. The displacement current is the reverse to that in the charging process. It corresponds to the relaxation of the charge carriers within the capacitor. This curve progression agrees with the general discharging behavior of real capacitors.

The asymmetry of the cyclic voltammogram reveals that the LV-EWOD electrode behaves differently in the charging and discharging process. This can mainly result from the material characteristics of anodic $\mathrm{Ta}_{2} \mathrm{O}_{5}$, an almost perfect isolator ${ }^{108}$. As a valve metal oxide, anodic $\mathrm{Ta}_{2} \mathrm{O}_{5}$ can ideally block anodic current, when the applied voltage is below $50 \%$ of the anodization voltage for the $\mathrm{Ta}_{2} \mathrm{O}_{5}$ formation ${ }^{152}$. In the $\mathrm{CV}$ measurement here, the maximal voltage is $0.4 \mathrm{~V}$. This is far below the half of $30 \mathrm{~V}$, at which the $\mathrm{Ta}_{2} \mathrm{O}_{5}$ was anodized for using in the EWOD electrode. Thus, in the charging process the displacement current was observed exclusively. However, the anodic $\mathrm{Ta}_{2} \mathrm{O}_{5}$ can nearly but not completely block the cathodic current ${ }^{152}$. This may result in the dissipation with a marginal faradaic current flowing through the anodic $\mathrm{Ta}_{2} \mathrm{O}_{5}$ in the discharging process. 
Based on the typical capacitor behavior revealed by the cyclic voltammogram, the hypothesis at the section beginning can be confirmed: It is appropriate to model the MDS as a capacitor in the equivalent circuit (Figure 2.12 b) as postulated in Chapter 2.2.2.1.

Second, the $\boldsymbol{C} / \boldsymbol{A}$ of the MDS was determined through EIS with the simultaneous optical imaging to compare with the $\boldsymbol{C} / \boldsymbol{A}$ of the ODTS- $\mathrm{Ta}_{2} \mathrm{O}_{5}$-bilayer (Chapter 4.1.3.3). Based on that, the capacitance of the thin oil layer in the interfacial region can be characterized indirectly.

Experimentally, the MDS was obtained by changing the measuring ambient medium from air to oil for the $3 \mu \mathrm{L}$ droplet of $200 \mathrm{mM} \mathrm{KCl}$ resting on the LV-EWOD electrode (Chapter 3.3.2). Identically to the experiments for determining the $\boldsymbol{C} / \boldsymbol{A}$ of the ODTS- $\mathrm{Ta}_{2} \mathrm{O}_{5}$-bilayer, EIS measurements were performed using the single sinusoidal signal with a voltage amplitude of $0.5 \mathrm{~V}$ and an offset of $0.5 \mathrm{~V}$ in the frequency range from $100 \mathrm{~Hz}$ to $10 \mathrm{kHz}$. The optical imaging was applied to measure the contact area between the droplet and the electrode to normalize the capacitance to the $\boldsymbol{C} / \boldsymbol{A}$. The data were analyzed with the equivalent circuit model, which consists of a resistor for the droplet resistance and a capacitor for the total capacitance of the dielectric layer stack.

At this measuring condition, the object under investigation was considered to be at its resting state without the EWOD effect, since the applied voltage is below the threshold to facilitate the EWOD effect (Figure 4.23, Figure 4.24, Chapter 4.3.3.1).

The determined $\boldsymbol{C} / \boldsymbol{A}$-values are plotted against frequency in Figure 4.12. The solid ODTS$\mathrm{Ta}_{2} \mathrm{O}_{5}$-bilayer shows higher and more constant $\boldsymbol{C} / \boldsymbol{A}$-values over the frequency range (filled squares, replotted from Figure 4.10) than those corresponding to the oil-ODTS- $\mathrm{Ta}_{2} \mathrm{O}_{5}$-stack (empty squares in Figure 4.12).

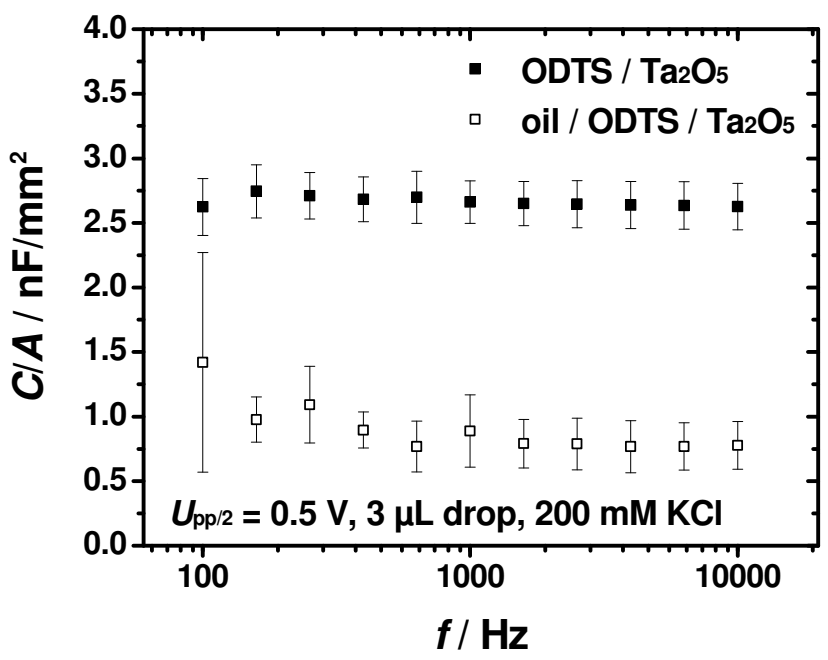

Figure 4.12. Capacitance per unit area of the MDS $(N=5)$ and that of the ODTS- $\mathrm{Ta}_{2} \mathrm{O}_{5}$-bilayer $\left(N=3\right.$, Figure 4.10) determined with EIS using a single sinusoidal signal $\left(U_{\mathrm{pp} / 2}=0.5 \mathrm{~V}\right)$ on a $3 \mu \mathrm{L}$ drop of $200 \mathrm{mM} \mathrm{KCl}$ resting on the LV-EWOD electrode. 
However, concerning the oil layer as a fluid dielectric layer in the MDS, $\boldsymbol{C} / \boldsymbol{A}$-value of the MDS is generally constant except the first data point at $100 \mathrm{~Hz}$. This data point was regarded as an outlier due to its high uncertainty. The nearly constant $\boldsymbol{C} / \boldsymbol{A}$ of the oil-ODTS- $\mathrm{Ta}_{2} \mathrm{O}_{5}$-stack indicates the oil layer to be stable at this low voltage with varied frequency.

The mean $\boldsymbol{C} / \boldsymbol{A}$-value of the oil-ODTS- $\mathrm{Ta}_{2} \mathrm{O}_{5}$-stack was determined without the first data point to be $(0.818 \pm 0.268) \mathrm{nF} / \mathrm{mm}^{2}$. Its coefficient of variance $(\mathrm{cv})$ yields $32.8 \%$. In comparison, the ODTS- $\mathrm{Ta}_{2} \mathrm{O}_{5}$-bilayer has a higher $\boldsymbol{C} / \boldsymbol{A}$-value of $(2.66 \pm 0.16) \mathrm{nF} / \mathrm{mm}^{2}$ but a lower $\mathrm{cv}$-value of $6.0 \%$. The significant discrepancy in the $\mathrm{cv}$-values results from the large variability of the oil layer thickness. This may be influenced by the insertion position and depth of the platinum electrode in the droplet.

Based on the capacitive feature of the MDS (Figure 4.11) and its higher $\boldsymbol{C} / \boldsymbol{A}$ than that of the solid ODTS- $\mathrm{Ta}_{2} \mathrm{O}_{5}$-bilayer (Figure 4.12), the oil layer is suggested to act as a capacitor as well. Moreover, the overlapping structure of the oil layer on top and the solid ODTS- $\mathrm{Ta}_{2} \mathrm{O}_{5}$-bilayer beneath supports the equivalent circuit model postulated for the MDS (Figure 2.12b, Chapter 2.2.2.1): the series connection of one capacitor for the oil layer and two capacitors for the solid bilayer. Therefore, the oil layer can be calculated with the $\boldsymbol{C} / \boldsymbol{A}$-value of the MDS and that of the ODTS-Ta $\mathrm{O}_{5}$-bilayer (Figure 4.12) according to the following correlation:

$$
\frac{A}{C_{\text {oil }}}=\frac{A}{C_{\text {MDS }}}-\frac{A}{C_{\text {bilayer }}}
$$

The $\boldsymbol{C} / \boldsymbol{A}$-value of oil layer yields $(1.18 \pm 0.56) \mathrm{nF} / \mathrm{mm}^{2}$. This characterizes the initial capacitive state of the oil layer between the droplet and the LV-EWOD electrode without EWOD effect.

\subsubsection{Oil Layer Thickness}

The oil layer entrapped between the droplet and the LV-EWOD electrode is characterized for its average thickness. As a fluid dielectric, this interfacial oil layer has a variable thickness depending on the radial position relative to the center of the droplet. The average thickness was determined by modeling the entire oil layer as a plate capacitor with a constant thickness and through calculation based on the following correlation (transformed from Eq. 2.11):

$$
d=\varepsilon_{0} \cdot \varepsilon_{d} \cdot \frac{A}{C}
$$

To characterize the entrapped oil layer at its initial resting state without EWOD effect, its thickness was determined according to Eq. 4.5 with the $C / A$-value of $(1.18 \pm 0.56) \mathrm{nF} / \mathrm{mm}^{2}$ (Chapter 4.1.4.2), the permittivity of vacuum $\left(\varepsilon_{0}\right)$ and the relative permittivity value of tetradecane $\left(\varepsilon_{\boldsymbol{d}}=2.03\right){ }^{153}$. This yields a thickness of $(15.2 \pm 7.2) \mathrm{nm}$. The large standard deviation of the oil layer thickness indicates its high variability. This variability is suggested to depend on the insertion position and the insertion depth of the platinum electrode in the droplet. 
Moreover, a thickness below $20 \mathrm{~nm}$ is far beyond the resolution limit of a light microscope (>200 $\mathrm{nm}$ ) based on the light diffraction (Abbe's) limit ${ }^{154}$. This thin oil layer might be detectable with the STED (stimulated emission depletion) nanoscope ${ }^{155}$ or Raman spectroscopy ${ }^{156-157 .}$ However, using these measurement techniques would require (i) complicated and expensive experimental setups, (ii) a transparent EWOD electrode for the detection in z-direction. In comparison, the indirect determination method applied in this study provides a low-cost and convenient opportunity to obtain the thickness information of a fluid layer in the $\mathrm{nm}$ range and is suitable for a non-transparent electrode.

\subsubsection{Conclusions}

This section focuses on the layer-by-layer characterization of the fabricated LV-EWOD electrode in its working ambience of oil. The object of interest, the multilayer dielectric stack (MDS, Figure 4.1), consists of three dielectric layers: the solid $\mathrm{Ta}_{2} \mathrm{O}_{5}$ layer, the solid ODTS layer and the fluid oil layer. Each layer was investigated with regard to its surface property, its layer property and its material property. The corresponding EWOD-relevant parameters are quantitatively determined for each layer and summarized in the following table:

Table 4.4. Overview table of the EWOD-relevant parameters determined for each dielectric layer in the MDS. $R_{\mathrm{RMS}}$ : root-mean-square roughness, $\mathrm{CAH}$ : contact angle hysteresis, $\theta_{0}$ : contact angle without voltage application.

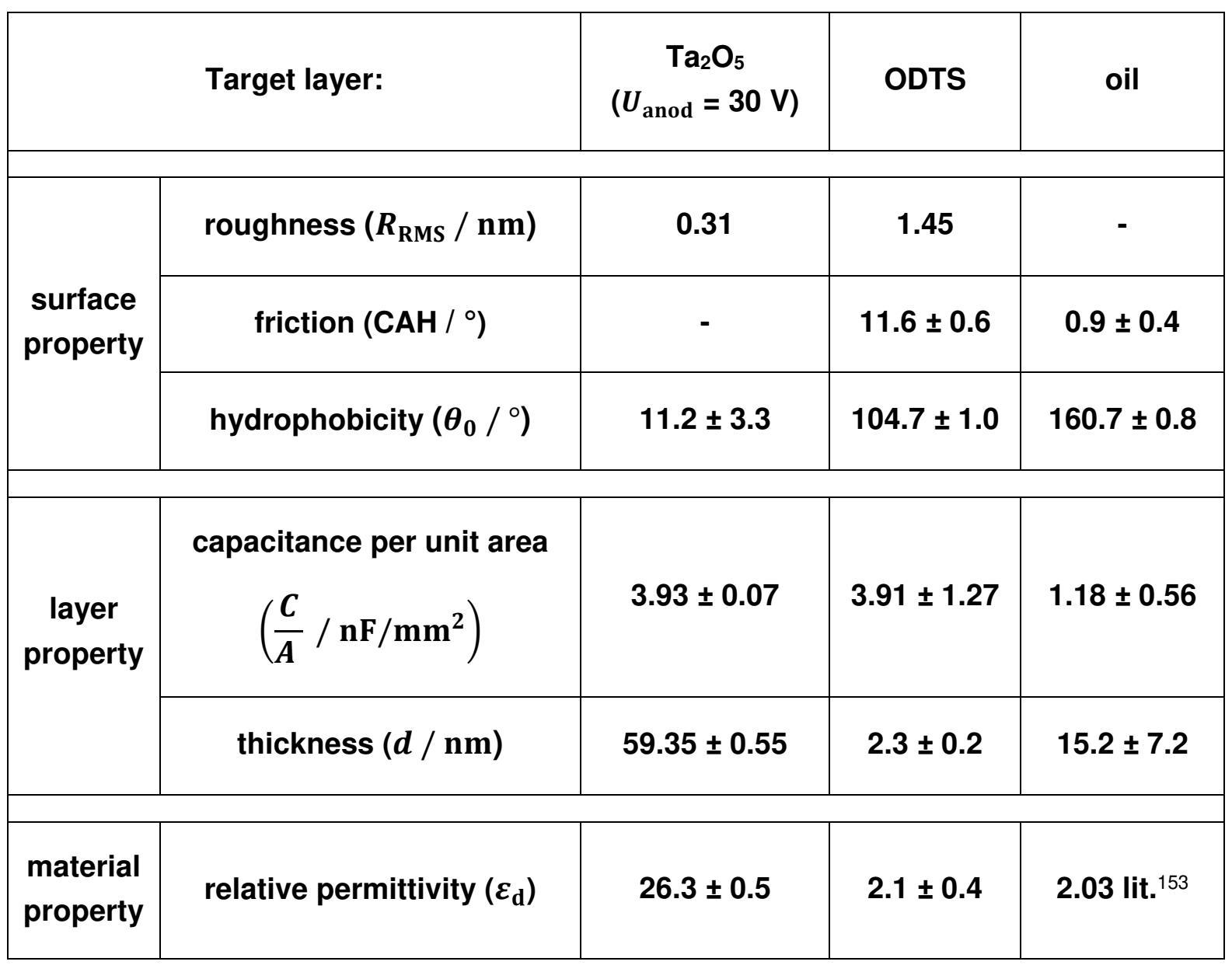


In this study, a thin anodic $\mathrm{Ta}_{2} \mathrm{O}_{5}$ layer fabricated through anodization at $30 \mathrm{~V}$ is evaluated as the best candidate to be the initial dielectric layer in the MDS. This layer features a smooth and hydrophilic surface and a homogeneous layer thickness. Moreover, it is outstanding due to the high reproducibility and reliability of its capacitance per unit area and of its high relative permittivity.

The anodization voltage is indicated to be an essential factor for adjusting the properties of the $\mathrm{Ta}_{2} \mathrm{O}_{5}$ layer. The linear increase of the $\mathrm{Ta}_{2} \mathrm{O}_{5}$ layer thickness with the anodization voltage is characterized with a growth coefficient of $(1.94 \pm 0.05) \mathrm{nm} / \mathrm{V}$. As of the anodization voltage of $30 \mathrm{~V}$, the $\mathrm{Ta}_{2} \mathrm{O}_{5}$ layer thickness is predictable and reproducible according to this linear dependence. The capacitance per unit area of the $\mathrm{Ta}_{2} \mathrm{O}_{5}$ layer is confirmed to correlate with the reciprocal value of the anodization voltage. The relative permittivity, an important material property of the anodic oxide layer, shows independence of the anodization voltage from $30 \mathrm{~V}$ upwards.

The anodic $\mathrm{Ta}_{2} \mathrm{O}_{5}$ layer has proved advantageous over native tantalum oxide because of its higher relative permittivity and its better isolating property to avoid the leakage current and to increase the breakdown strength.

Based on the $\mathrm{Ta}_{2} \mathrm{O}_{5}$ layer, a self-assembled ODTS layer consists as the middle layer in the MDS. The thickness of the monolayer characterizes this silane layer. The silanization successfully converts the electrode surface from hydrophilic to hydrophobic. This surface chemistry change is suggested to be responsible for the subsequent entrapment of a thin oil layer as the fluid dielectric top layer in the MDS.

By replacing air with oil as the ambient medium, a thin oil layer is indicated to occupy the space between the droplet and the electrode. This interfacial scenario results in the significantly enlarged contact angle of the droplet in its resting state and the largely reduced surface friction against the droplet motion (Table 4.4). These two parameters highlight the benefits of an oil environment for a successful low-voltage EWOD effect. Moreover, the application of an oil environment can prevent the $\mu \mathrm{L}$-sized droplets from evaporation, to assure the measurement reliability and to enable the long-time investigation. This advantageous operation ambience is also applied in the 2 -fluid-phase (water-oil) systems for the microfluidics ${ }^{158}$, liquid lenses ${ }^{15}$ and EWOD displays ${ }^{159}$.

Furthermore, the capacitance per unit area $(\boldsymbol{C} / \boldsymbol{A})$ is an important EWOD-relevant parameter due to its role in the areal electric potential energy (Eq. 2.9), which induces the EWOD effect. In this study, the $\boldsymbol{C} / \boldsymbol{A}$-values were determined for the $\mathrm{Ta}_{2} \mathrm{O}_{5}$-ODTS-bilayer and the MDS without EWOD effect respectively: $(2.66 \pm 0.16) \mathrm{nF} / \mathrm{mm}^{2}$ and $(0.818 \pm 0.268) \mathrm{nF} / \mathrm{mm}^{2}$. The former value was as reference subsequently involved in the estimation of the oil layer thickness at the EWOD effect (Chapter 4.3.2). 
In this study, a few key parameters, such as the relative permittivity of $\mathrm{Ta}_{2} \mathrm{O}_{5}$ layer, that of the silane layer as well as the thickness of the thin oil layer between the droplet and the electrode, are experimentally difficult to assess by direct analysis methods. To determine these parameters, a methodology involving indirect investigations and physical correlations based on equivalent circuit models was applied.

One methodology in this study is the independent investigation by ellipsometry and by EIS with simultaneous imaging followed by calculation based on the plate capacitor model. With this methodology, the relative permittivity, an essential material property, can be individually specified for diverse fabrication conditions. This enhances the quality control of the $\mathrm{Ta}_{2} \mathrm{O}_{5}$ layer and the silane layer. Moreover, this enables different fabrication conditions to be compared and the appropriate conditions to be selected, e.g. for preparing the $\mathrm{Ta}_{2} \mathrm{O}_{5}$ layer used in the low-voltage EWOD study.

Concerning the thin oil layer, its thickness in the nm-range as well as its location between an aqueous drop and a non-transparent solid electrode hinder its thickness to be determined with the usual optical analysis methods. A low-cost and convenient solution to overcome this obstacle is using the indirect methodology by measuring the layer capacitance and converting it to the thickness information. It involves EIS with simultaneous macroscopic imaging, the capacitors-in-series model for the MDS, the plate capacitor model for the oil layer and the literature value of the oil relative permittivity. With this indirect method, the oil layer thickness can be determined at the droplet resting state as reference (Chapter 4.1.4.3) and be monitored online during the EWOD process (Chapter 4.3.2). 


\subsection{Frequency Dependence of EWOD}

Partial results in this chapter have been published in Li, Ying-Jia and Cahill, Brian P.,

"Frequency Dependence of Low-Voltage Electrowetting Investigated by Impedance Spectroscopy", Langmuir 2017, doi: 10.1021/acs.langmuir.7b03049.

To understand and assess the frequency dependence of the EWOD effect at low alternating voltages, this study focuses on the influence of frequency on the three EWOD-relevant variables in the Young-Lippmann equation (Eq. 2.2). They are the voltage drop on the dielectric layer in the EWOD electrode $(\boldsymbol{U})$, the capacitance per unit area of the dielectric layer $(\boldsymbol{C} / \boldsymbol{A})$ and the contact angle under voltage $\left(\boldsymbol{\theta}_{\mathbf{u}}\right)$. Furthermore, the influence of frequency is analyzed for the correlation of these three EWOD-relevant variables under various measurement conditions.

The system under investigation is a sessile droplet of aqueous $\mathrm{KCl}$ solution resting on the LVEWOD electrode in the oil surrounding. The fabrication and the characterization of the LVEWOD electrode were presented in Chapter 3.1, 3.2 and 4.1 respectively. The region of interest is the droplet-electrode-interface including an entrapped oil layer as the fluid dielectric. This interfacial region is described as the multilayer dielectric stack (MDS, Figure 2.11, Chapter 2.2.1.3). The droplet and the MDS are electrically modelled with a developed equivalent circuit (Figure $2.12 \mathrm{~b}$, Chapter 2.2.2.1). For the alternating voltage applications, an adapted Young-Lippmann equation (Eq. 2.32) is postulated in Chapter 2.2.2.3.

The influence of frequency was investigated online using the integrated LV-EWOD-EIS system and high-speed imaging system (Chapter 3.3.1). It facilitates simultaneous EWOD excitation, EIS measurement and optical analysis of the droplet geometry. In this study, single sinusoidal signals were applied to the system under investigation with two variable quantities: frequency $(100 \mathrm{~Hz}-1 \mathrm{MHz})$ and amplitude voltage $(0.5 \mathrm{~V}-5 \mathrm{~V})$. A third variable of the measuring condition was the salt concentration of the droplet varied within the physiological-relevant range $(6.25 \mathrm{mM}-200 \mathrm{mM} \mathrm{KCl})$.

In this section, the three EWOD-relevant variables in the adapted Young-Lippmann equation (Eq. 2.32) were individually and systematically addressed as shown in Figure 4.13.

The online impedimetric response of EWOD (Chapter 4.2.1) serves to determine $\boldsymbol{U}_{(\mathbf{R M S}) \text { MDS }}$, the effective voltage drop on the MDS, (Chapter 4.2.2) and $\boldsymbol{C}_{\mathrm{MDS}}$, the MDS capacitance. The optical characterization of the droplet geometry yields $\boldsymbol{\theta}_{\mathbf{u}}$, the contact angle under voltage, (Chapter 4.2.4) and $\boldsymbol{A}$, the droplet / electrode contact area. From $\boldsymbol{C}_{\text {MDS }}$ and $\boldsymbol{A}$, the MDS capacitance per unit area, $\boldsymbol{C}_{\mathrm{MDS}} / \boldsymbol{A}$, is determined (Chapter 4.2.3). Based on the three EWODrelevant variables, the conversion efficiency of the electric potential energy to EWOD effect is evaluated with reference to the correlation coefficient $\boldsymbol{\beta}$ in the adapted Young-Lippmann equation (Chapter 4.2.5). 


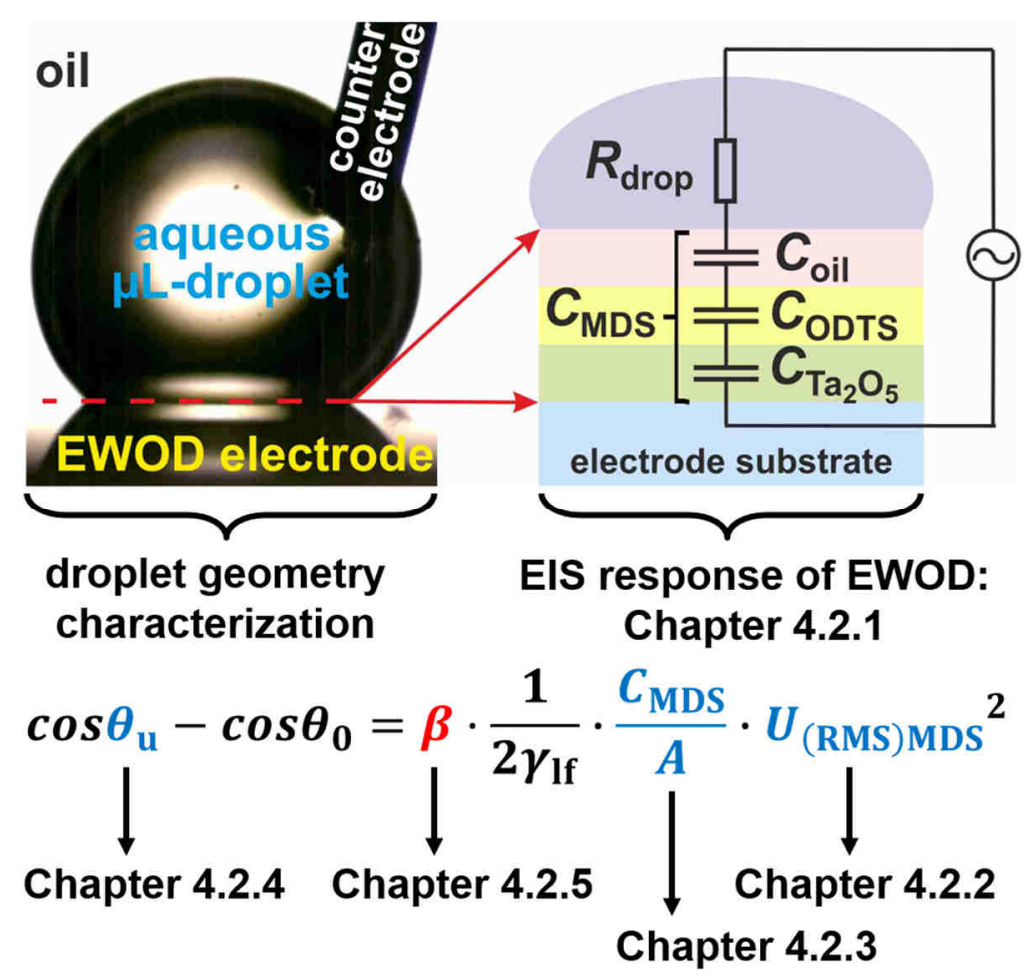

Figure 4.13. Section overview with the optical image of the system under study, its equivalent circuit model (reprint of Figure $2.12 \mathrm{~b}$, Chapter 2.2.2.1) and the adapted Young-Lippmann equation (Eq. 2.32). Three EWOD-relevant variables (blue): effective voltage drop on the MDS $\left(U_{(\mathrm{RMS}) \mathrm{MDS}}\right)$, MDS-capacitance per unit area $\left(C_{\mathrm{MDS}} / A\right)$ and contact angle under voltage $\left(\theta_{\mathrm{u}}\right)$. $\beta$ : correlation coefficient for assessment of the EWOD effect.

\subsubsection{Online Impedance Spectroscopy of EWOD}

By means of the integrated LV-EWOD and EIS, the impedimetric response of the system under investigation can be traced online during EWOD. In this study, the impedance spectra were recorded at frequencies from $1 \mathrm{MHz}$ to $100 \mathrm{~Hz}$, at voltage amplitudes from $0.5 \mathrm{~V}$ to $5 \mathrm{~V}$ and with different droplet $\mathrm{KCl}$ concentrations from $6.25 \mathrm{mM}$ to $200 \mathrm{mM}$ (Chapter 3.3.2). The impedimetric response was recorded under the diverse operating conditions to investigate the equivalent circuit model (Figure $2.12 \mathrm{~b}$ ) for representing the system under study during EWOD. Based on this equivalent circuit model, the impedance spectra were analyzed for determining the voltage drop on the MDS (Chapter 4.2.2) and the MDS-capacitance.

The equivalent circuit model describes the droplet as a resistive component and the entire MDS as a capacitive component (Chapter 2.2.2.1). Concerning its configuration as an RC combination in series, the impedance spectra should show two different responses: one frequency-independent resistive response and one frequency-dependent capacitive response at all measurement conditions. The latter is based on the frequency dependence of the impedance contribution from a capacitive component: $\boldsymbol{X}_{C}=\frac{1}{2 \pi f C}$ (Eq. 2.30). The validity of the equivalent circuit is supported by comparison with the impedance spectra obtained at various voltages (Figure $4.14 \mathrm{a}$ ) and for a range of saline concentrations (Figure $4.14 \mathrm{~b}$ ). 

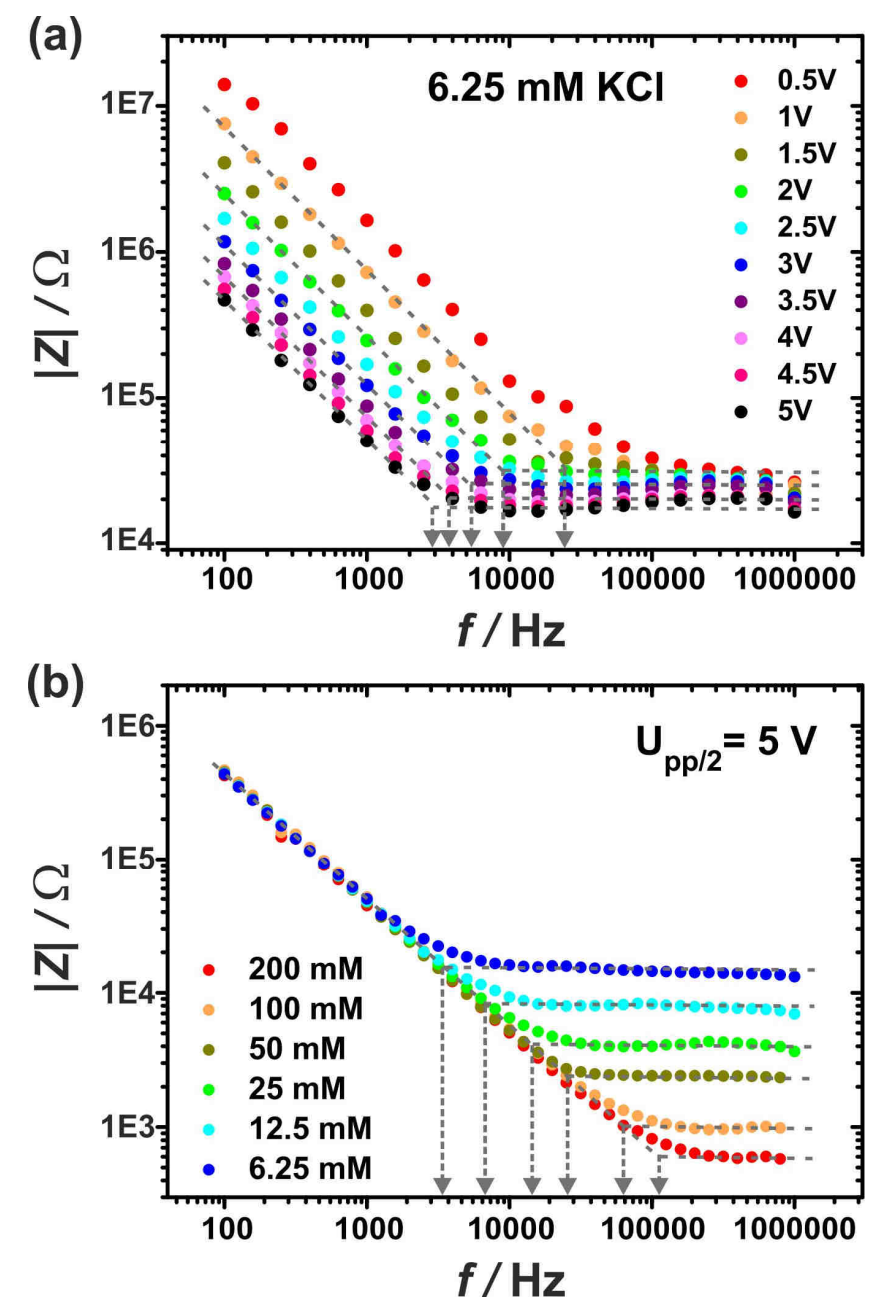

Figure 4.14. Impedance spectra of impedance modulus against frequency (a) during EWOD at varied voltage amplitudes $\left(U_{\mathrm{pp} / 2}=0.5-5 \mathrm{~V}\right)$ on a droplet of $6.25 \mathrm{mM} \mathrm{KCl}$ and (b) during EWOD of droplets with diverse $\mathrm{KCl}$ concentrations $(c=6.25-200 \mathrm{mM})$ at the voltage amplitude of $5 \mathrm{~V}$. Grey arrows: critical frequencies dividing the impedance spectra in the capacitive and the resistive response of the system under investigation.

For EWOD at varied voltages, representative impedance spectra (Figure $4.14 \mathrm{a}$ ) are demonstrated with measurements on droplets with a constant $\mathrm{KCl}$ concentration of $6.25 \mathrm{mM}$. Despite the difference in the applied voltage, each spectrum shows two characteristic impedimetric responses separated by a critical frequency. The critical frequency is pointed out by a grey arrow in Figure 4.14 (a) for each measurement at a $\boldsymbol{U}_{\mathbf{p p} / 2}$ with the integer values.

Below the critical frequency, the impedance modulus shows an inverse linear correlation to the frequency at each voltage. This impedimetric response is characteristic for a capacitor. Above the critical frequency, the impedance modulus appears to be independent of frequency. This impedimetric behavior is typical for a resistor. These two characteristic responses appear in the impedance spectra at all the applied voltages. This result confirms the hypothesis postulated above. Hence, the system under investigation can be electrically modeled as an $\mathrm{RC}$ combination. 
Moreover, the impedance spectra show that the applied voltage significantly influences the capacitive response of the system under investigation. In contrast, the voltage does not significantly affect the resistive response.

For EWOD of droplets with diverse $\mathrm{KCl}$ concentrations, representative impedance spectra are exhibited for a constant voltage of $5 \mathrm{~V}$ (Figure $4.14 \mathrm{~b}$ ).

Despite the difference in the $\mathrm{KCl}$ concentration, each impedance spectrum reveals the two characteristic responses as well: the capacitive response below the critical frequency and the resistive response above the critical frequency. Concerning the resistive response, the impedance modulus decreases with increasing the salt concentration in the droplet. Concerning the capacitive response at a constant voltage, all impedance spectra present a coincident inverse linear correlation between the impedance modulus and the frequency.

The concentration dependence of the resistive response implies the droplet to be the resistive element in the system under investigation. The influence of the $\mathrm{KCl}$ concentration on the resistive response can be explained as below: For the investigated concentration range, the concentration of the strong electrolyte $(\mathrm{KCl})$ proportionally correlates with its specific conductivity. ${ }^{49}$ The specific conductivity correlates inversely with the solution resistance, which is the predominant component of the frequency-independent real part of the impedance.

The concentration independence and the coincidence of the capacitive response (Figure 4.14 b) indicate that, the capacitive element in the system under study is independent of the $\mathrm{KCl}$ concentration in the measuring range. This result suggests that, the contribution of the double layer capacitance at the wire-droplet-interface and that at the droplet-MDS-interface to the entire capacitance of the system under study can be neglected. A detailed proof with a comparison between the MDS capacitance and the double layer capacitance is provided as the supporting material in the publication ${ }^{145}$. Therefore, the MDS can be considered as the capacitive element in the system under study.

The results presented above suggest the validity of the equivalent circuit model for representing the system under study during EWOD effect at the measuring conditions.

\subsubsection{Voltage Drop on MDS Influenced by Frequency and Concentration}

According to the equivalent circuit model (Figure $2.12 \mathrm{~b}$ ), the capacitive MDS can only store a part of the electric potential energy depending on the voltage drop across it $\left(\boldsymbol{E}_{\mathrm{el}}=\frac{\mathbf{1}}{\mathbf{2}} \cdot \boldsymbol{C}_{\mathrm{MDS}} \cdot \boldsymbol{U}_{\mathrm{MDS}}^{2}\right)$. According to the Young-Lippmann equation (Eq. 2.2), the electric potential energy per unit area stored in the $\operatorname{MDS}\left(\boldsymbol{E}_{\mathrm{el}} / \boldsymbol{A}=\frac{1}{2} \boldsymbol{C}_{\mathrm{MDS}} \cdot \boldsymbol{U}_{\mathrm{MDS}}^{2}\right.$, Eq. 2.9) induces the EWOD effect. Due to its quadratic contribution to the $\boldsymbol{E}_{\mathrm{el}} / \boldsymbol{A}$, the voltage drop on the MDS is implied to essentially influence the strength of the EWOD effect.

This voltage was determined with the voltage division principle (Eq. 2.29) and the impedance spectra analysis of the impedance modulus and phase. Concerning the alternating voltage 
application, the effective voltage value is calculated as the root-mean-square value (Eq. 2.31). The percentage of the voltage drop on MDS $\left(\frac{U_{(\mathrm{RMS}) \mathrm{MDS}}}{U_{\mathrm{RMS}}} \%\right)$ was investigated for the frequency dependence at various voltages (Figure 4.15 a) and with different saline concentrations (Figure 4.15 b). A hypothesis about the frequency influence is postulated as below: Since the system under study is electrically modeled as an $\mathrm{RC}$ combination in series, the voltage drop on the capacitive MDS should show a frequency dependence like the first-order low-pass filters $^{30,55}$ (Figure 2.13, Chapter 2.2.2.1) in the electrical engineering.

The percentage of the voltage drop across MDS shows a comparable frequency dependence at various voltages with a constant saline concentration (example: $6.25 \mathrm{mM} \mathrm{KCl}$ ) in the droplet (Figure $4.15 \mathrm{a}$ ): In the low frequency region, $100 \%$ of the applied voltage drops across the MDS. With the frequency increase, the percentage of voltage drop on MDS decreases gradually toward $0 \%$.
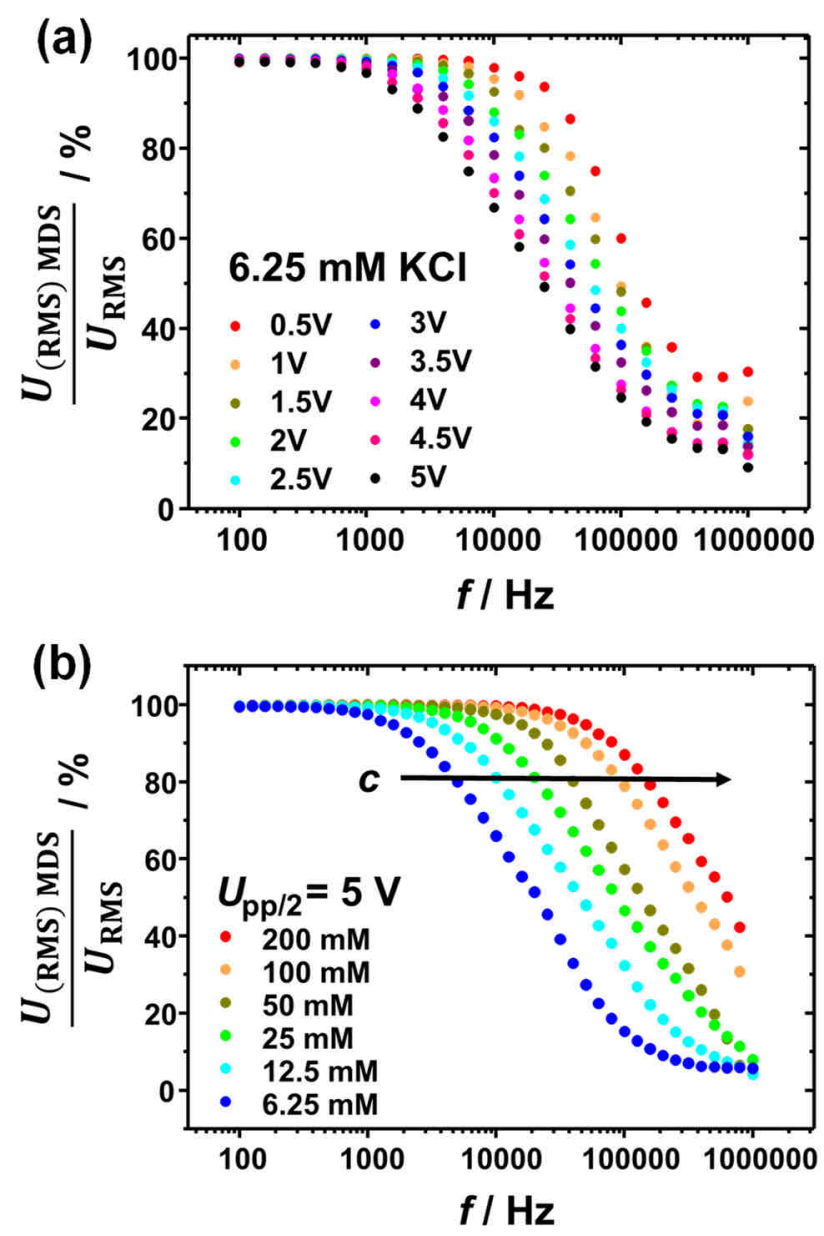

Figure 4.15. Percentage of the RMS-voltage drop on the MDS versus frequency (a) for EWOD at alternating voltages from $0.5 \mathrm{~V}$ to $5 \mathrm{~V}$ on a droplet of $6.25 \mathrm{mM} \mathrm{KCl}$ and (b) for EWOD on droplets with $\mathrm{KCl}$ concentrations from $200 \mathrm{mM}$ to $6.25 \mathrm{mM}$ at the alternating voltage of $5 \mathrm{~V}$. Black arrow with $c$ : increase of $\mathrm{KCl}$ concentration. 
This frequency dependence reflects a typical electrical low-pass filter behavior. Moreover, this low-pass filter behavior of the voltage percentage is observed for all measured saline concentrations (Figure $4.15 \mathrm{~b}$ ). These results confirm the hypothesis and support the validity of the equivalent circuit model to represent the system under study as a series RC-circuit.

With increasing saline concentration (black arrow in Figure $4.15 \mathrm{~b}$ ), the voltage percentage curve shifts to the higher frequencies and thus the low-pass frequency range extends. This influence can be explained by the following correlation: The saline concentration contributes negatively to the droplet resistance (detailed explanation in Chapter 4.2.1), which affects the total impedance (Eq. 2.23) and the voltage division (Eq. 2.29). Thus, the saline concentration is indicated to be a determining factor of the frequency range of the low-pass filter.

\subsubsection{MDS Capacitance Influenced by Oil Layer and Frequency}

As the origin of the EWOD effect, the electric potential energy per unit area $\left(\boldsymbol{E}_{\mathrm{el}} / \boldsymbol{A}=\frac{\mathbf{1}}{\mathbf{2}} \cdot \frac{\boldsymbol{C}_{\mathrm{MDS}}}{\boldsymbol{A}} \cdot \boldsymbol{U}_{\mathrm{MDS}}^{\mathbf{2}}\right.$, Eq. 2.9) is contributed by the MDS capacitance per unit area $\left(\boldsymbol{C}_{\mathrm{MDS}} / \boldsymbol{A}\right)$.

Based on the equivalent circuit model (Figure $2.12 \mathrm{~b}$ ), the MDS-capacitance $\left(\boldsymbol{C}_{\text {MDS }}\right)$ was determined by analyzing the impedance spectra of modulus $(|\boldsymbol{Z}|)$ and phase $(\emptyset)$ with $\boldsymbol{X}_{C_{\mathrm{MDS}}}=-|\boldsymbol{Z}| \cdot \sin \emptyset$ (Eq. 2.18, Eq. 2.20) and $\boldsymbol{X}_{\boldsymbol{C}_{\mathrm{MDS}}}=\frac{1}{2 \pi f C_{\mathrm{MDS}}}$ (Eq. 2.29). The area (A) refers to the contact area between the droplet and the LV- EWOD electrode and was obtained through image analysis of the droplet-electrode interfacial region.

Moreover, the capacitance per unit area, $\boldsymbol{C}_{\text {MDS }} / \boldsymbol{A}$, was used to exclude the influence of the contact area change. The influence of voltage and of frequency on $\boldsymbol{C}_{\text {MDS }}$ / $\boldsymbol{A}$ during EWOD is shown in Figure 4.16 for a $6.25 \mathrm{mM} \mathrm{KCl}$ droplet.

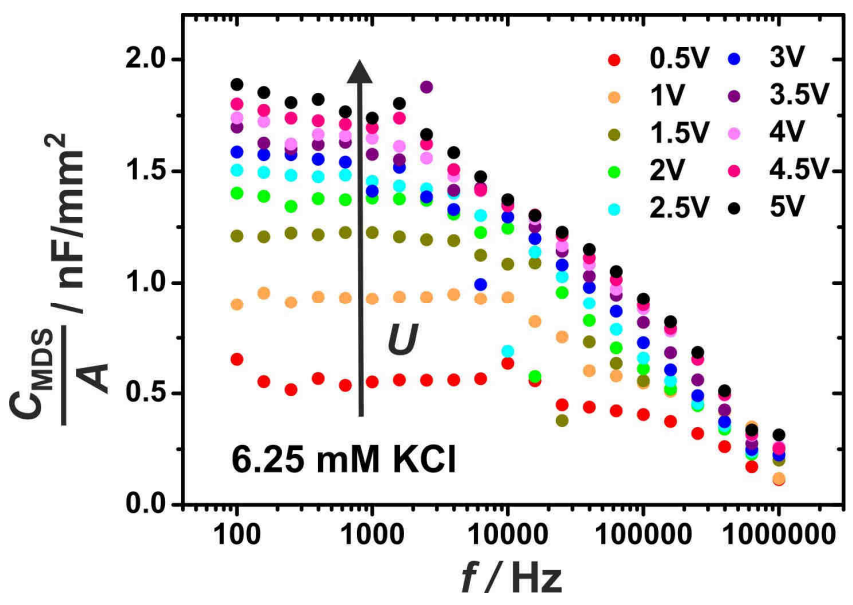

Figure 4.16. MDS capacitance per unit contact area to the droplet versus frequency for EWOD at alternating voltages from $0.5 \mathrm{~V}$ to $5 \mathrm{~V}$ on a droplet of $6.25 \mathrm{mM} \mathrm{KCl}$. Black arrow with $U$ : increase of the voltage. 
Each $\boldsymbol{C}_{\text {MDS }}$ / $\boldsymbol{A}$ curve exhibits a decrease with the frequency, especially in the high frequency region. Since MDS works as a capacitor, upon applying a voltage on the system under investigation, the charging process in MDS is time-related. At these high frequencies, the corresponding period values are lower than the time constant $(\boldsymbol{\tau}=\boldsymbol{R C})$ of the system under investigation. The charging process is incomplete, and less electric potential energy can be stored.

Moreover, $\boldsymbol{C}_{\mathrm{MDS}}$ / $\boldsymbol{A}$ significantly increases with the voltage, especially in the low frequency region. Based on the correlation, $\frac{C}{A}=\frac{\varepsilon_{0} \varepsilon_{d}}{d}$ (Eq. 2.11), this parameter is affected by the thicknesses of the dielectric layers consisting in the MDS. The thickness of its two solid layers, the $\mathrm{Ta}_{2} \mathrm{O}_{5}$-layer and the ODTS-layer, are invariable during EWOD. In contrast, the oil layer as a fluid between the droplet and the electrode can be deformed during EWOD. Its thickness should decrease with the increasing electrostatic pressure ${ }^{48}$. This means, that the oil layer should be squeezed out of the space between the droplet and the electrode by increasing the voltage. This hypothesis is supported by the observed increase of the MDS capacitance per unit area with the applied voltage.

\subsubsection{Contact Angle Influenced by Voltage and Frequnecy}

The electrowetting behavior of a droplet is characterized by its voltage-induced contact angle decrease and the corresponding contact area increase. The contact angle under voltage, $\boldsymbol{\theta}_{\mathrm{u}}$, as marked in a droplet image in Figure 4.17 (a), is the parameter used to study the influence of voltage and frequency on the electrowetting effect.

The EWOD-induced contact angle change at voltages lower than $5 \mathrm{~V}$ is shown in Figure 4.17 (b). A contact angle change of $30^{\circ}$ in the oil was observed at the AC voltage amplitude of $5 \mathrm{~V}\left(6.12 \mathrm{~V}_{\mathrm{RMS}}\right)$ during the frequency decrease from $1 \mathrm{MHz}$ to $100 \mathrm{~Hz}$. This lowvoltage electrowetting performance is comparable or better than the results from other research groups that used $\mathrm{Ta}_{2} \mathrm{O}_{5}$ insulating layers. To reach the same contact angle change in air, an EWOD electrode combining $\mathrm{Ta}_{2} \mathrm{O}_{5}$ with Teflon-AF required $13 \mathrm{~V}_{\mathrm{DC}}{ }^{72}$. EWOD electrodes with $\mathrm{Ta}_{2} \mathrm{O}_{5}$ and Cytop ${ }^{\mathrm{TM}}$ demanded $6 \mathrm{~V}_{\mathrm{DC}}$ to overcome the actuation threshold ${ }^{71}$ or to approach a contact angle change of $19^{\circ}$ in air ${ }^{72}$. The lower voltage demand reported here can be attributed to the much lower thickness of silane monolayers in comparison with fluoropolymers layers coated by spin coating.

Since the system under investigation shows a low-pass filter behavior (Figure 4.15), less voltage is dropped across $C_{\text {MDs }}$ at high frequencies. Thus, at low frequencies and high voltages, more electric potential energy is stored in $\boldsymbol{C}_{\text {MDS }}$ and the electrowetting is expected to be enhanced. 
The representative contact angle behavior at varied frequency (example: $6.25 \mathrm{mM} \mathrm{KCl}$ droplet) is presented in Figure 4.17 (b).

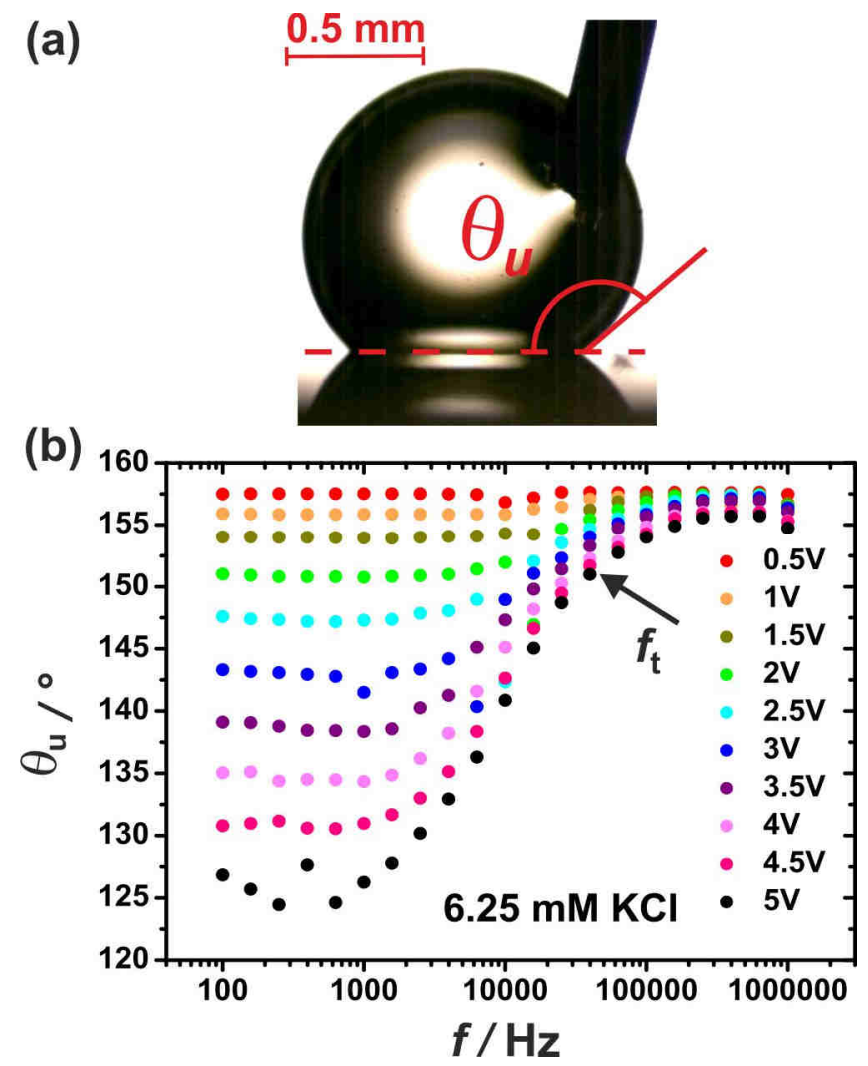

Figure 4.17. (a) Optical image of a $3 \mu \mathrm{L}$ droplet in tetradecane. $\theta_{\mathrm{u}}$ : contact angle under voltage. (b) Contact angle under voltage versus frequency for the electrowetting at alternating voltages from $0.5 \mathrm{~V}$ to $5 \mathrm{~V}$ on a droplet of $6.25 \mathrm{mM} \mathrm{KCl}$. Black arrow with $f_{t}$ : frequency threshold.

At the alternating voltages above and equal to $2 \mathrm{~V}$, the contact angle is significantly affected by the frequency. By lowering the frequency from $1 \mathrm{MHz}$ to $100 \mathrm{~Hz}$, the contact angle remains unchanged at the high frequencies, decreases at a frequency threshold (black arrow in Figure 4.17 b) and reaches saturation at the low frequencies. Moreover, the contact angle change depends on the applied voltage by reducing the final saturated contact angle. These indicate, the electrowetting can only occur below a frequency threshold and it is enhanced by lowering frequency and by increasing voltage.

Furthermore, at the alternating voltages below $2 \mathrm{~V}$, frequency has no significant influence on the contact angle. This indicates that the voltage drop across MDS and the electric potential energy stored in the MDS are too low to cause significant electrowetting. 


\subsubsection{Young-Lippmann Equation for AC Applications}

The three EWOD-relevant variables $\left(\boldsymbol{U}_{(\mathbf{R M S}) \mathrm{MDS}}, \frac{\boldsymbol{C}_{\mathrm{MDS}}}{\boldsymbol{A}}, \boldsymbol{\theta}_{\mathbf{u}}\right)$ in the adapted Young-Lippmann equation (Eq. 2.32, Chapter 2.2.2.3) are directly or indirectly influenced by frequency, voltage and salt concentration in the droplets, as shown in the previous chapters (Chapter 4.2.2 Chapter 4.2.4).

How frequency influences the Young-Lippmann equation, is analyzed here with a plot of the electrowetting number $\left(\frac{C_{\mathrm{MDS}}}{2 \gamma_{\mathrm{If}} A} \boldsymbol{U}_{(\mathrm{RMS}) \mathrm{MDS}}^{2}\right)^{35}$ against the EWOD-induced contact angle change $\left(\cos \theta_{\mathbf{u}}-\cos \theta_{\mathbf{0}}\right)$. The slope yields the correlation coefficient $\boldsymbol{\beta}$. It indicates the percentage of the electric potential energy contributing to the electrowetting.

A plot of the adapted Young-Lippmann equation (Eq. 2.32) is shown for the electrowetting at various frequencies from $100 \mathrm{~Hz}$ to $1 \mathrm{MHz}$ on a droplet of a physiologically relevant salt concentration (100 $\mathrm{mM} \mathrm{KCl})$ as a representative example (Figure 4.18).
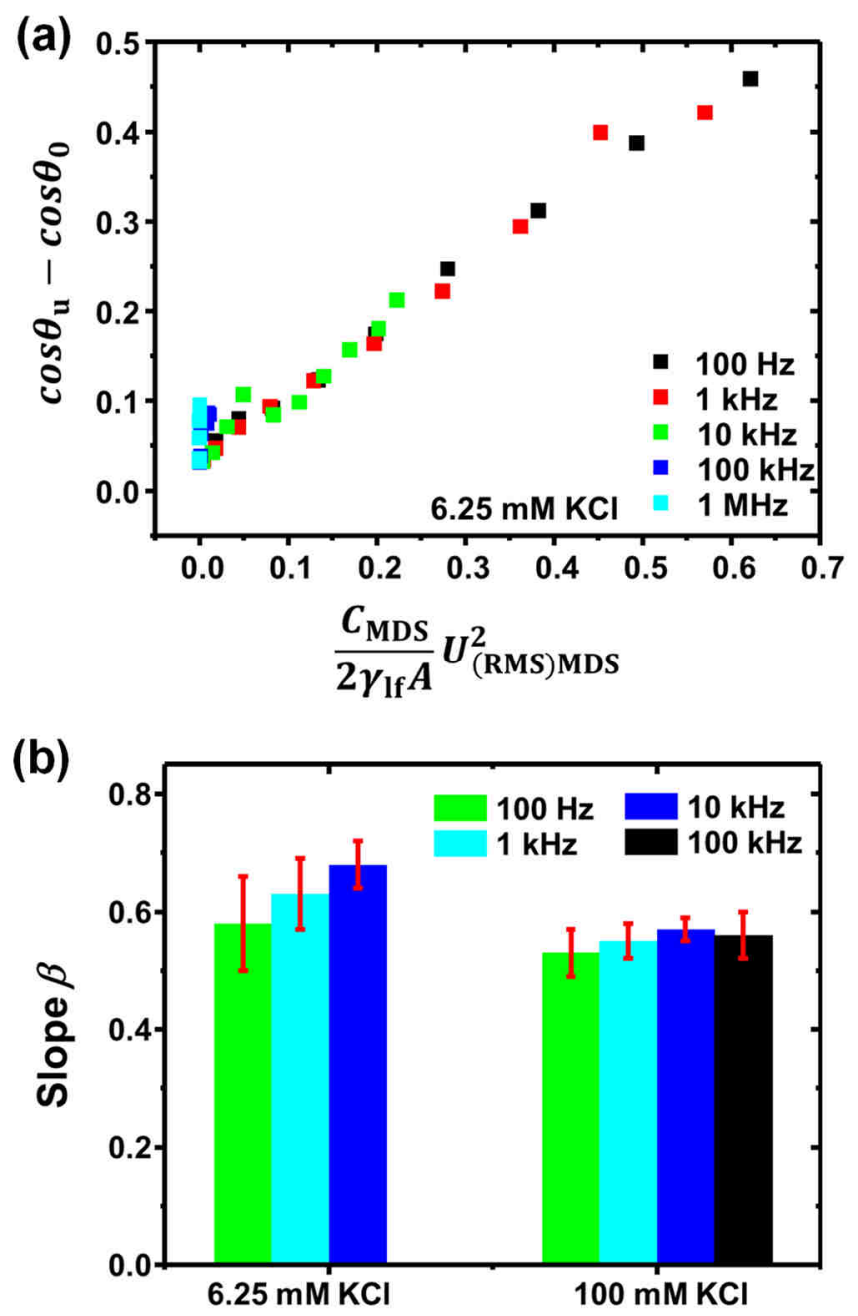

Figure 4.18. (a) Plot of the adapted Young-Lippmann equation (Equation 2.32) for the EWOD at frequencies from $100 \mathrm{~Hz}$ to $1 \mathrm{MHz}$ on a droplet of $100 \mathrm{mM} \mathrm{KCl}$. (b) Correlation coefficient $\beta$ for droplets of $100 \mathrm{mM} \mathrm{KCl}$ and $6.25 \mathrm{mM} \mathrm{KCl}$ at frequencies where $\beta<1(N=3)$. 
The adjusted coefficient of determination $\left(\overline{\boldsymbol{R}}^{2}\right)$ and the slope ( $\boldsymbol{\beta}$, Figure $4.18 \mathrm{~b}$, right bars, $\boldsymbol{N}=3$ ) were determined through a linear fitting for the lower frequencies $(100 \mathrm{~Hz} \leq f \leq 100 \mathrm{kHz})$. All the $\overline{\boldsymbol{R}}^{2}$ values are very close to one $\left(\overline{\boldsymbol{R}}^{2}>0.98\right)$ and the significance of each linear correlation is confirmed by a Student's t-test ${ }^{160}$ with $99 \%$ confidence. This indicates a significant linear correlation between the electrowetting number and $\cos \boldsymbol{\theta}_{\mathbf{u}}-\boldsymbol{c o s} \boldsymbol{\theta}_{\mathbf{0}}$ in this frequency regime.

Furthermore, the right bars in Figure $4.18 \mathrm{~b}$ reveal the comparable slopes $(\boldsymbol{\beta})$ of the linear correlations in the lower frequency range from $100 \mathrm{~Hz}$ to $100 \mathrm{kHz}$. Their average value is $0.55 \pm 0.02$. The significant linear correlations and the comparable $\boldsymbol{\beta}$ values suggest that the electrowetting behavior at alternating voltages can be well described by the adapted YoungLippmann equation (Eq. 2.32) with a stable $\boldsymbol{\beta}$ value in this frequency region. Moreover, the $\boldsymbol{\beta}$ value below one indicates that the electric potential energy stored in the dielectrics only partially contributes to the electrowetting. The residual energy may be converted to electromechanical work for the oil layer deformation and to dissipation during EWOD. Staicu and Mugele have investigated the deformation of an entrapped oil layer in a comparable measurement setup. The oil film thickness is described by an extension of the Landau-Levich law regarding the electrostatic pressure ${ }^{36}$.

In contrast, the dataset of the highest frequency $(f=1 \mathrm{MHz})$ reveals no significant linear correlation between $\cos \boldsymbol{\theta}_{\mathbf{u}}-\boldsymbol{\operatorname { c o s }} \boldsymbol{\theta}_{\mathbf{0}}$ and the electrowetting number based on a Student's twotailed t-test with $95 \%$ confidence limit. This suggests that EWOD effect is not the main cause of contact angle change at this high frequency.

This frequency-based difference shown in the correlation behavior reveals that, electrowetting occurs below a frequency threshold. The frequency-based relationship between electrowetting and dielectrophoresis was studied by Jones et al.44-45: electrowetting dominates at lower frequencies while dielectrophoresis dominates at higher frequencies. Due to the asymmetric electrode configuration (Figure 2.11) in the measurement setup here, the aqueous droplet experiences an inhomogeneous electric field during electrowetting. For a comparable system as reported here, Shapiro et al. ${ }^{106}$ have demonstrated the non-uniform electric potential scaling inside a slightly resistive droplet and in its underneath EWOD electrode. Since the voltage mainly drops across the droplet rather than across the MDS at high frequencies, the gradient of the electric field may be sufficiently high to cause a significant volume polarization force ${ }^{46}$ on the droplet and thus induce dielectrophoresis here.

In addition, at a low salt concentration of $6.25 \mathrm{mM} \mathrm{KCl}$, the correlation in the adapted YoungLippmann equation behaves similarly: Below a frequency threshold of $10 \mathrm{kHz}$, significant linear correlations deliver comparable slope values below one $(\boldsymbol{\beta}=0.63 \pm 0.05)$ at various frequencies (Figure $4.18 \mathrm{~b}$, left bars). This confirms that electrowetting dominates in the low frequency range. Moreover, this indicates that the frequency and the salt concentration have no significant influence on the correlation in the adapted Young-Lippmann equation. 
However, the frequency region for electrowetting is limited by a lower threshold at the low salt concentration $\left(f_{\mathrm{t}}=10 \mathrm{kHz}\right)$ than at the high salt concentration $\left(f_{\mathrm{t}}=100 \mathrm{kHz}\right)$. This implies that the salt concentration in the droplet regulates the frequency threshold, below which the adapted Young-Lippmann equation can well describe the electrowetting behavior at alternating voltages.

\subsubsection{Conclusions}

The frequency dependence of the EWOD effect on application of alternating voltages is the focus of study presented in this section. The key prerequisite for this study is the simultaneous implementation of EWOD excitation, impedimetric investigation and imaging analysis. This was fulfilled with the high-speed imaging instrument in association with the LV-EWOD-EIS system. By means of frequency-domain EIS using a sinusoidal voltage signal with variable frequency and amplitude, the EWOD effect was induced on a droplet with various salt concentrations on the low-voltage EWOD electrode in oil. Its impedimetric response at diverse measurement conditions confirms the validity of the equivalent circuit model for electrically representing the object under study: the droplet and the MDS. The latter includes two solid dielectric layers in the EWOD electrode and a fluid oil layer between the droplet and the electrode. The basic Young-Lippmann equation was adapted to account for alternating voltages by specifying the effective voltage and added with a correlation coefficient $(\boldsymbol{\beta})$ to indicate the EWOD efficiency.

The frequency dependence of the three EWOD-related variables in the adapted YoungLippmann equation was investigated: the effective voltage drop across MDS, the MDScapacitance per unit area and the contact angle under voltage. First, the frequency dependence of the voltage drop across MDS indicates a low-pass filter behavior; the low-pass frequency region can be regulated by the salt concentration. Second, the MDS-capacitance per unit area shows a voltage-dependence at low frequencies due to the deformation of the oil layer between the droplet and the electrode during electrowetting. Meanwhile, the decrease of the MDS-capacitance per unit area at high frequencies reveals that the time limitation may result in the incomplete storage of the electric potential energy in the MDS. Third, the frequency dependence of the contact angle under voltage is visible above a voltage threshold and below a frequency threshold.

Finally, the correlation between both sides of the adapted Young-Lippmann equation was studied with regard to frequency dependence. Below a frequency threshold, electrowetting dominates and can be well described by the adapted Young-Lippmann equation independently of frequency and salt concentration. In this frequency region, the correlation coefficient $(\boldsymbol{\beta})$ is less than one, thus the electric potential energy stored in MDS is indicated to only partially contribute to EWOD effect. 


\subsection{Non-Linearity and Dynamics in EWOD System ${ }^{\mathrm{XIV}}$}

In this section, the non-linearity and dynamics of electrowetting and dewetting were studied with focus on the electric potential energy per unit area $\left(\boldsymbol{E}_{\mathrm{el}} / \boldsymbol{A}\right)$ for the EWOD system with oil as the ambient medium. In this study, the EWOD system (Figure 4.19 a) was electrically excited, impedimetrically characterized using dynamic EIS and optically analyzed by highspeed imaging. The EWOD system was a $3 \mu \mathrm{L}$-droplet of $200 \mathrm{mM} \mathrm{KCl}$ immersed in oil and resting on the LV-EWOD electrode with two solid dielectric layers (Chapter 3.1): an ODTS monolayer coating and an anodic $\mathrm{Ta}_{2} \mathrm{O}_{5}$ layer $\left(\boldsymbol{U}_{\text {anod }}=30 \mathrm{~V}\right)$. The objects of interest are the droplet and the interfacial oil layer between the droplet and the electrode. The latter is considered as the top layer in the MDS. The applied voltage stimulus was a symmetric staircase signal $\left(\boldsymbol{U}_{\text {off,min }}=0.3 \mathrm{~V}, \boldsymbol{U}_{\text {off,max }}=5.8 \mathrm{~V}, \boldsymbol{T}=1 \mathrm{~s}\right)$ with a square signal $\left(\boldsymbol{U}_{\mathrm{pp} / 2}=0.3 \mathrm{~V}\right.$, $\boldsymbol{f}=10 \mathrm{kHz}$ ) integrated at each voltage step (Figure 2.7, Chapter 2.1.2.2, Chapter 3.3.3). The ascending voltage ramp in the staircase stimulus induces electrowetting and the descending ramp leads to the dewetting. For both processes, the time-resolved response of the EWOD system was traced in terms of following parameters (Figure $4.19 \mathrm{~b}$ ): the contact angle $\left(\boldsymbol{\theta}_{\mathbf{u}}\right)$, the contact area $(\boldsymbol{A})$ of the droplet and the area-related capacitance $(\boldsymbol{C})$ of the multilayer dielectric stack (MDS).

(a)

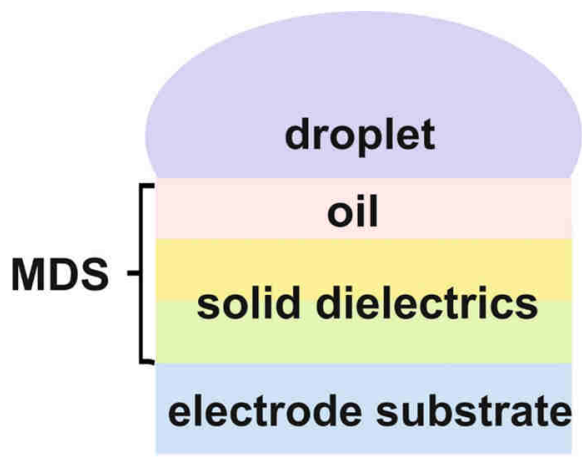

(b)

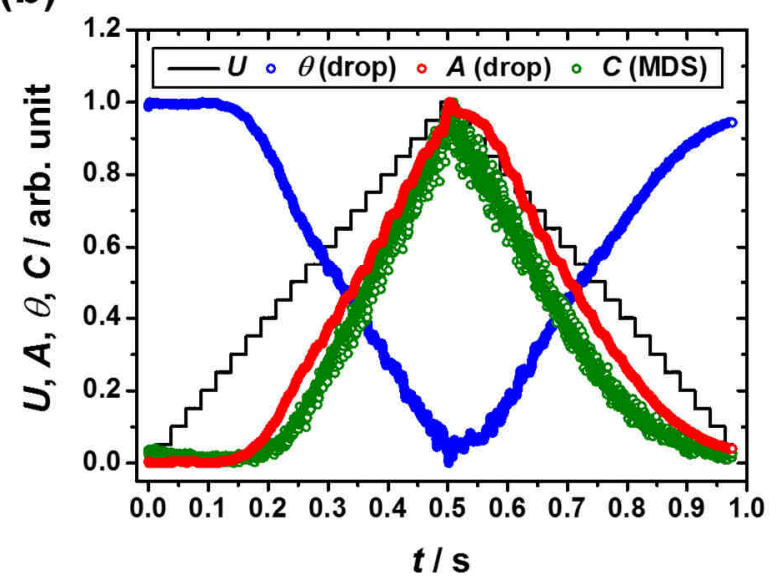

Figure 4.19. (a) EWOD system model (Chapter 2.2.1.3): an aqueous saline droplet on the MDS (multilayer dielectric stack) consisting of an interfacial oil layer and solid dielectrics (b) Overview of the time-resolved response of the contact area (red), the contact angle (blue) of the droplet and the area-related MDS-capacitance (green) to the staircase voltage stimulus (black). The arbitrary unit is defined for each parameter as the ratio to the difference between its maximum and its minimum value.

\footnotetext{
${ }^{X I V}$ All results in this section were produced in cooperation with the research group at IBA for the funding project, "Impedanzspektroskopische Bioanalytik - schnell und hochparallel", 2016 FGR 0040.
} 
This section begins with the voltage dependence of the origin of the EWOD effect, which is the electric potential energy per unit area stored in the MDS, and the correlated capacitance per unit area of the MDS (Chapter 4.3.1). The non-linear behavior and the hysteresis feature of the EWOD system at electrowetting and dewetting is characterized by the thickness change of the interfacial oil layer (Chapter 4.3.2) and the droplet deformation with a view of the voltage threshold and the contact angle hysteresis (Chapter 4.3.3). The dynamics of the EWOD system is studied with the characteristic time of the contact area alternation based on the timeresolved measurements of the electrowetting and the dewetting process (Chapter 4.3.4).

\subsubsection{Electric Potential Energy and MDS-Capacitance}

As the Young-Lippmann equation (Eq. 2.2) implies, the electric potential energy per unit area $\left(\boldsymbol{E}_{\mathrm{el}} / \boldsymbol{A}\right)$ stored in the MDS yields the EWOD effect. This electric potential energy per unit area depends on the MDS-capacitance per unit area $\left(\boldsymbol{C}_{\mathrm{MDS}} / \boldsymbol{A}\right)$ and the applied voltage $(\boldsymbol{U})$ according to Eq. 2.9, $\frac{\boldsymbol{E}_{\mathrm{el}}}{\boldsymbol{A}}=\frac{\mathbf{1}}{\mathbf{2}} \cdot \frac{\boldsymbol{C}}{\boldsymbol{A}} \cdot \boldsymbol{U}^{2}$.

To find out the $\boldsymbol{C} / \boldsymbol{A}$ in this study, the area-related MDS capacitance $\left(\boldsymbol{C}_{\mathrm{MDS}}\right)$ was determined by means of dynamic EIS for equidistant voltage steps between $0.3 \mathrm{~V}$ and $5.8 \mathrm{~V}$ in an ascending ramp followed by a descending ramp. The corresponding droplet contact area $(\boldsymbol{A})$ was obtained through the synchronized optical imaging (Chapter 3.3.3).

Based on the $\boldsymbol{C}_{\mathrm{MDS}} / \boldsymbol{A}$, the electric potential energy per unit area $\left(\boldsymbol{E}_{\mathrm{el}} / \boldsymbol{A}\right)$ is calculated with the voltage offset $\left(\boldsymbol{U}_{\text {off }}\right)$ for each step in the staircase signal according to Eq. 2.9. A representative voltage dependence of $\boldsymbol{E}_{\mathrm{el}} / \boldsymbol{A}$ is shown for the ascending ramp in red and for the descending ramp in blue (Figure 4.20).

The non-linear $\boldsymbol{E}_{\mathrm{el}} / \boldsymbol{A}$-voltage dependence in the ascending ramp and that in the descending ramp are generally in coincidence with each other despite slight discrepancy. Within the voltage region from $2.2 \mathrm{~V}$ to $4.1 \mathrm{~V}, \boldsymbol{E}_{\mathrm{el}} / \boldsymbol{A}$ has lower values in the ascending ramp than in the descending ramp. In contrast, higher $\boldsymbol{E}_{\mathrm{el}} / \boldsymbol{A}$ values were determined in the ascending ramp than in the descending ramp between $4.4 \mathrm{~V}$ and $5.3 \mathrm{~V}$. 


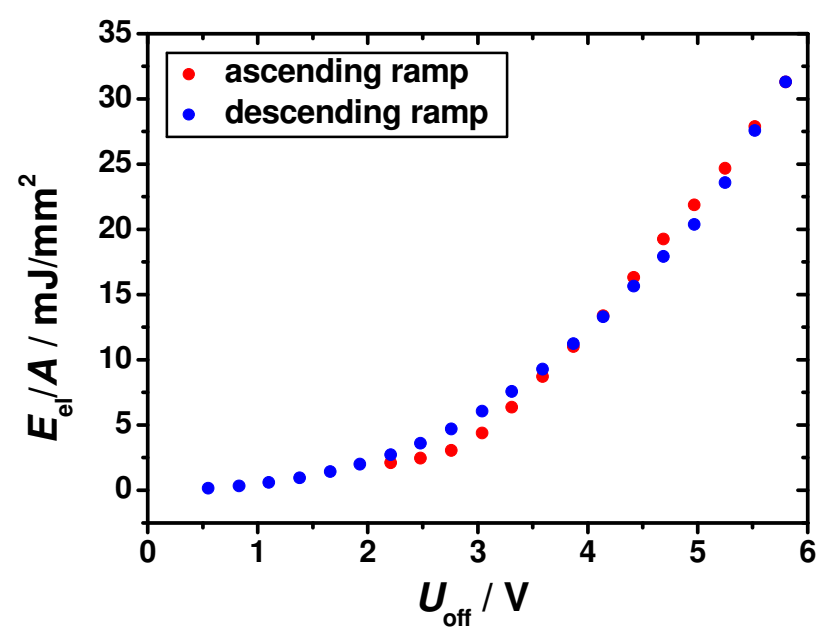

Figure 4.20. Non-linear voltage dependence of the electric potential energy per unit area $\left(E_{\mathrm{el}} / A\right)$ in the ascending (red) and in the descending (blue) voltage ramp. $U_{\text {off: }}$ voltage offsets of the staircase stimulus. ${ }^{x v}$

The discrepancy of the $\boldsymbol{E}_{\mathrm{el}} / \boldsymbol{A}$ behavior in the electrowetting and in the dewetting process can be elucidated by the different $\boldsymbol{C}_{\mathrm{MDS}} / \boldsymbol{A}$-voltage dependence in the ascending ramp and in the descending ramp shown in Figure 4.21.

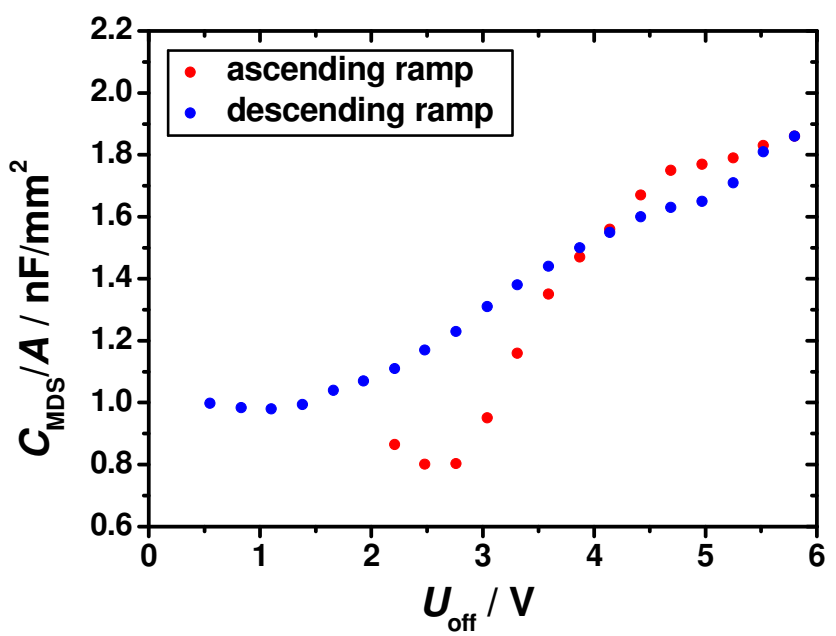

Figure 4.21. Non-linear responses of the MDS-capacitance per unit area $\left(C_{\text {MDs }} / A\right)$ to the ascending (red) and to the descending (blue) voltage ramp. $U_{\text {off }}$ : voltage offsets of the staircase stimulus.

In the ascending ramp, data points of the $\boldsymbol{C}_{\mathrm{MDS}} / \boldsymbol{A}$ are presented above the voltage threshold (about 2 V, Figure 4.23 and Figure 4.24, Chapter 4.3.3.1) for inducing EWOD effect. The omission of the data points in the measurement beginning is explained in Footnote XV.

\footnotetext{
${ }^{\mathrm{XV}}$ At the low voltages in the ascending ramp $(0.3-1.9 \mathrm{~V})$, six data points of the capacitance were not appropriately determined due to the signal drift at the beginning of the EIS measurement, which did not originate from the object under study. Since $\boldsymbol{E}_{\mathrm{el}} / \boldsymbol{A}$ and $\boldsymbol{C} / \boldsymbol{A}$ were calculated with the capacitance, their data points in this voltage region are hence omitted in all relevant figures.
} 
Starting with its minimum value around $2.5 \mathrm{~V}$, the $C_{\mathrm{MDS}} / \boldsymbol{A}$ shows a steep rise followed by an attenuated increase in the ascending ramp. In the descending ramp, the $\boldsymbol{C}_{\text {MDS }} / \boldsymbol{A}$ decreases continuously and almost linearly with the voltage.

This $\boldsymbol{C}_{\mathrm{MDS}} / \boldsymbol{A}$-response to the applied voltage sequence is expected. As elucidated in the study of the frequency influence on the EWOD effect in Chapter 4.2.3, $\boldsymbol{C}_{\mathrm{MDS}} / \boldsymbol{A}$ should rise with the increasing voltage and decline with the reducing voltage. This voltage-influence on the $C_{\text {MDS }} / A$ can be reasoned with its correlation with the thickness of the oil layer $\left(\boldsymbol{d}_{\text {oil }}\right)$ in the MDS and the influence of the electrostatic pressure on $\boldsymbol{d}_{\text {oil }}$. The relationship between the $\boldsymbol{C}_{\text {MDs }} / \boldsymbol{A}$ and the $\boldsymbol{d}_{\mathbf{o i l}}$ can be expressed with Eq. 4.4 and Eq. 4.5, which are indicated by the equivalent circuit model (Figure $2.12 \mathrm{~b}$ ) representing the oil layer and the solid dielectrics as capacitors connected in series. The results about the oil layer deformation are provided in detail further on (Chapter 4.3.2).

Moreover, in comparison to the slight difference of the $\boldsymbol{E}_{\mathrm{el}} / \boldsymbol{A}$-voltage dependence in the electrowetting and the dewetting process, more significant difference is shown by the $\boldsymbol{C} / \boldsymbol{A}$ voltage dependence in the comparable voltage regions. This can be explained by the Eq. 2.9: Although $\boldsymbol{C} / \boldsymbol{A}$ contributes proportionally to $\boldsymbol{E}_{\mathrm{el}} / \boldsymbol{A}$, its influence is much less significant than the voltage influence on $\boldsymbol{E}_{\mathrm{el}} / \boldsymbol{A}$ with the power of two.

Therefore, despite the different response of $\boldsymbol{C} / \boldsymbol{A}$ in the electrowetting and dewetting process, the non-linear voltage dependence of $\boldsymbol{E}_{\mathrm{el}} / \boldsymbol{A}$ is comparable in both processes.

\subsubsection{Oil Layer Deformation}

To quantify the deformation of the interfacial oil layer during the electrowetting and the dewetting process, the oil layer thickness was evaluated. Due to its nm-scale, it is difficult to measure this parameter directly with the commonly used optical techniques. In this study, the oil layer thickness was indirectly determined from $\boldsymbol{C}_{\text {MDS }} / \boldsymbol{A}$ (Figure 4.21), by accounting for the capacitance of the solid $\mathrm{Ta}_{2} \mathrm{O}_{5}$-ODTS-bilayer $\left(2.66 \mathrm{nF} / \mathrm{mm}^{2}\right)$ using the plate capacitor model as described by Eq. 4.4 and Eq. 4.5. The approach and the procedure can be found in the previous sections (Chapter 4.1.4.2 and 4.1.4.3). A representative oil layer deformation with the electric potential energy per unit area $\left(\boldsymbol{E}_{\mathrm{el}} / \boldsymbol{A}\right)$ is shown in Figure 4.22 for the ascending ramp in red and for the descending ramp in blue. 


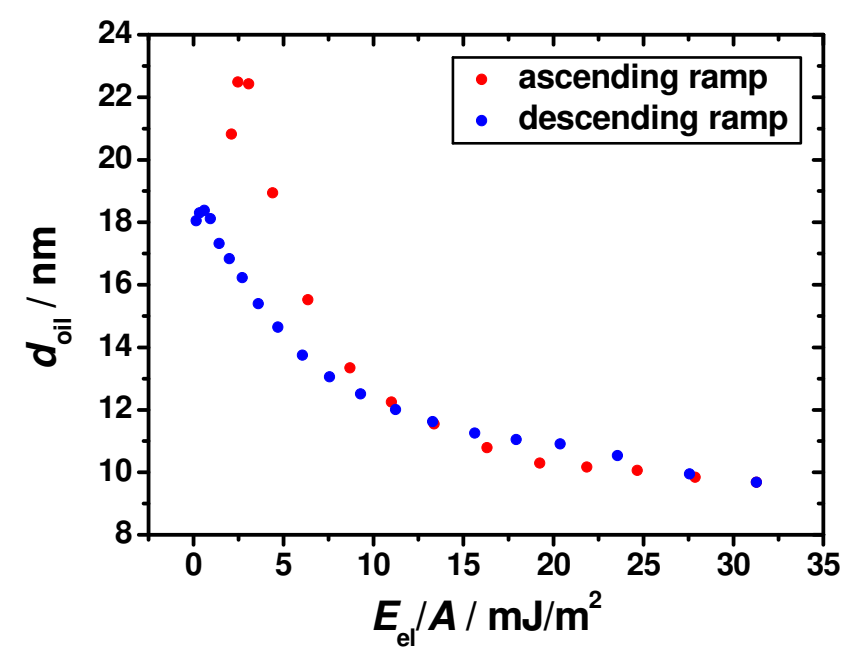

Figure 4.22. Non-linear variation of the oil layer thickness $\left(d_{\text {oil }}\right)$ with the electric potential energy per unit area $\left(E_{\mathrm{el}} / A\right)$ in the ascending (red) and in the descending (blue) voltage ramp.

In the ascending ramp, the oil layer reaches its maximal thickness at the low $\boldsymbol{E}_{\mathrm{el}} / \boldsymbol{A}$-level corresponding to the voltage threshold around $2 \mathrm{~V}$ (Chapter 4.3.3.1). This observation agrees with the literature report about a thick oil layer separating the water phase and a hydrophobic substrate at the droplet resting state ${ }^{35,161}$. The "thick" oil layer results from the substrate-wetting by the oil phase, since their microscopic interactions are stronger than those between the substrate and the aqueous phase. In this study, the oil phase should completely wet the hydrophobic ODTS-surface of the LV-EWOD electrode, mainly due to the long-range van der Waals interaction between the alkyl chains in the ODTS molecules and those in the oil (tetradecane) molecules (Chapter 4.1.4.1).

Beginning with its maximal value, the $\boldsymbol{d}_{\text {oil }}$ decays with the $\boldsymbol{E}_{\mathrm{el}} / \boldsymbol{A}$-increase constantly and approaches its minimum of $9.9 \mathrm{~nm}$ at the highest $\boldsymbol{E}_{\mathrm{el}} / \boldsymbol{A}$-level of $31 \mathrm{~mJ} / \mathrm{m}^{2}$. Upon the $\boldsymbol{E}_{\mathrm{el}} / \boldsymbol{A}$ decrease along the descending voltage ramp, the $\boldsymbol{d}_{\text {oil }}$ rises gradually and reaches a stable value of $18 \mathrm{~nm}$ around the $\boldsymbol{E}_{\mathrm{el}} / \boldsymbol{A}$-level of zero. This indicates that the droplet presses the oil out of the space between the droplet and the electrode by increasing the supply of the electric potential energy per unit area in the EWOD system. Reversely, the oil flows back with ceasing energy. This oil layer deformation can originate from the electrostatic forces ${ }^{47}$ changing the balance of the microscopic interactions ${ }^{162}$.

In the low $\boldsymbol{E}_{\text {el }} / \boldsymbol{A}$-range between $2.5 \mathrm{~mJ} / \mathrm{m}^{2}$ and $13 \mathrm{~mJ} / \mathrm{m}^{2}$, the oil layer presents a larger thickness change during the ascending voltage ramp than during the descending ramp. This reveals that the oil layer deforms more strongly during the electrowetting process than during the dewetting process in this low $\boldsymbol{E}_{\mathrm{el}} / \boldsymbol{A}$-region. In the high $\boldsymbol{E}_{\mathrm{el}} / \boldsymbol{A}$-range above $13 \mathrm{~mJ} / \mathrm{m}^{2}$, the difference in the oil layer deformation between the electrowetting and the dewetting process turns to be insignificant. The different non-linear $\boldsymbol{d}_{\text {oil }}$-change along the electrowetting and along the dewetting process suggests that, the oil layer deformation is a non-conservative process in the low $\boldsymbol{E}_{\mathrm{el}} / \boldsymbol{A}$-range and a nearly reversible process in the high $\boldsymbol{E}_{\mathrm{el}} / \boldsymbol{A}$-range.

Thus, the hysteresis in oil layer deformation occurs mainly at low energy levels. The involved dissipation ${ }^{10,35}$ may originate from the fluid friction in oil, namely the viscous effect. 
Furthermore, $\boldsymbol{d}_{\mathbf{o i l}}$-values obtained at the end of the descending ramp and at the beginning of the ascending ramp $(18.0 \mathrm{~nm}$ and $22.5 \mathrm{~nm})$ are slightly different, but comparable with the oil layer thickness determined for the droplet resting state $\left(\boldsymbol{d}_{\text {oil }}=15.2 \mathrm{~nm} \pm 7.2 \mathrm{~nm}\right.$ ) in Chapter 4.1.4.3.

\subsubsection{Droplet Deformation}

The droplet deformation is characterized by the contact angle under voltage $\left(\boldsymbol{\theta}_{\mathbf{u}}\right)$ and the contact area $(\boldsymbol{A})$ between the droplet and the EWOD electrode. These two parameters were traced online by high-speed optical imaging during the electrowetting and the dewetting process (Chapter 3.3.1, III and IV). In this study, the voltage threshold of EWOD effect is determined with both contact angle and contact area (Chapter 4.3.3.1). Moreover, the contact angle hysteresis indicates the energy loss in a cycle of the electrowetting and the dewetting process (Chapter 4.3.3.2).

\subsubsection{Low Voltage Threshold}

The voltage threshold for EWOD effect is defined as the lowest applied voltage, where the droplet starts to deform from its initial static state. In this study, the voltage threshold was yielded based on the response of the contact angle (Figure 4.23) as well as that of the contact area (Figure 4.24) to the voltage steps in the ascending ramp.

At the beginning of the voltage staircase, the contact angle remains constant despite the voltage application (Figure 4.23). The inhibited droplet deformation can be due to the inertia or fluid friction resulting from the bulk viscosity of the droplet and that of the surrounding oil. By increasing the voltage offset, the EWOD effect is shown by the reduction of the contact angle after the voltage threshold $\left(\boldsymbol{U}_{\mathbf{t}}\right)$ is overcome.

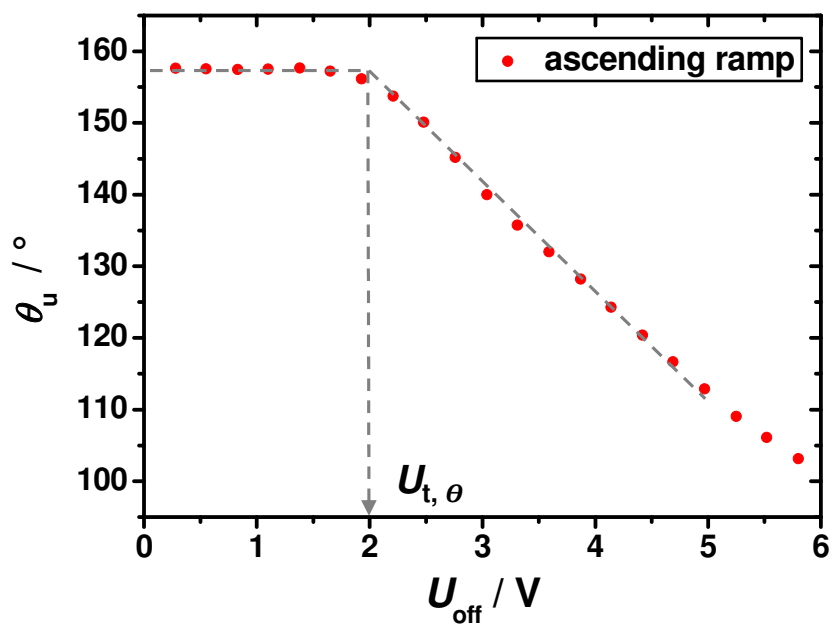

Figure 4.23. Voltage threshold $\left(U_{t, \theta}\right)$ of EWOD effect determined with the contact angle $\left(\theta_{\mathrm{u}}\right)$ response to the ascending voltage ramp. $U_{\text {off: }}$ voltage offsets of the staircase stimulus. 
As illustrated by the gray dashed lines in Figure 4.23, the voltage threshold corresponds to the intersection point of two extrapolated fitting lines: the base line of the initial static contact angle and the tangent line to the receding contact angle.

For a $3 \mu \mathrm{L}$ droplet of $200 \mathrm{mM} \mathrm{KCl}$ in oil, the voltage threshold $\left(\boldsymbol{U}_{\boldsymbol{t}, \boldsymbol{\theta}}\right)$ was determined to be $(1.93 \pm 0.16) \vee$ based on four measurements of the contact angle response.

Along the same ascending ramp, the droplet inertia behavior and the droplet spreading process upon overcoming the voltage threshold results in the corresponding contact area response: a stable phase with the initial constant area value followed by an increase (Figure 4.24). Here, the voltage threshold was determined with the same procedure used above in the case of the contact angle response.

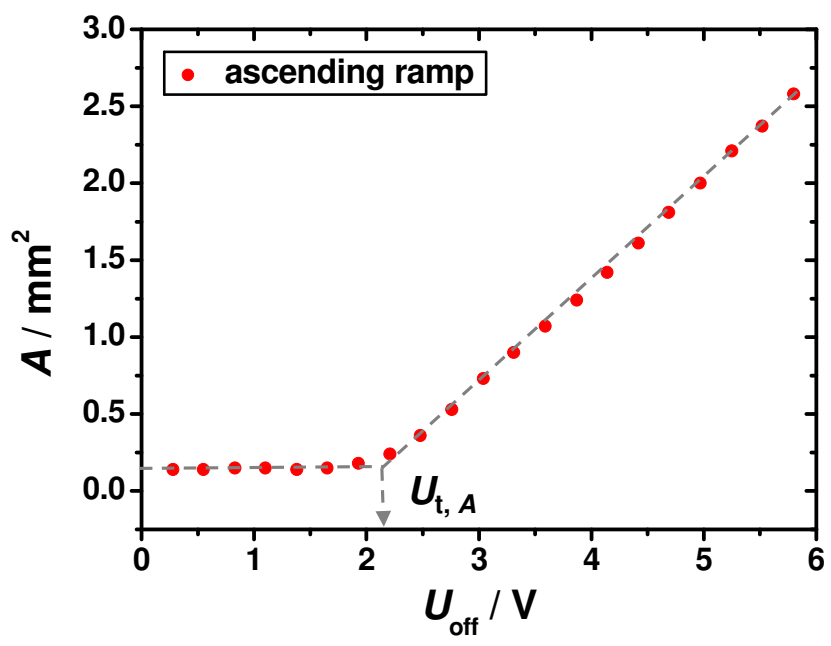

Figure 4.24. Voltage threshold $\left(U_{t, A}\right)$ of EWOD effect determined with the contact area $(A)$ response to the ascending voltage ramp. $U_{\text {off: }}$ voltage offsets of the staircase stimulus.

Four measurements of the contact area response yielded the voltage threshold $\left(\boldsymbol{U}_{\boldsymbol{t}, \boldsymbol{A}}\right)$ determined with the contact area of $(2.13 \pm 0.17) \mathrm{V}$.

The two voltage thresholds, $\boldsymbol{U}_{\boldsymbol{t}, \boldsymbol{\theta}}$ and $\boldsymbol{U}_{\boldsymbol{t}, \boldsymbol{A}}$, were determined for comparable low values. Concerning the advanced EWOD systems, this threshold is lower than the most achieved "low voltages" reported for example by Lin et al. $(7.2 \mathrm{~V})^{71}$, Khodayari et al. $(10 \mathrm{~V})^{163}$ and Moon et al. $(15 \mathrm{~V})^{164}$. The low voltage threshold yielded in this study confirms the achievement of a lowvoltage EWOD system.

The slightly higher value of $\boldsymbol{U}_{\boldsymbol{t}, \boldsymbol{\theta}}$ can result from the additional electrical energy required for overcoming the advancing contact angle, so that the force balance between the interfacial tensions and the surface friction force at the static state can be broken. Upon this condition, the contact line motion can occur. Due to the low contact angle hysteresis in this EWOD system (Chapter 4.1.4.1), the dissipation contributed by the surface friction against the contact line motion $^{36,165-166}$ is low, hence the voltage threshold for the contact angle change and that for the contact area change almost overlap. This minor contact line pinning is a feature of an effective EWOD system. 


\subsubsection{Contact Angle Hysteresis}

The hysteresis of droplet deformation in the electrowetting and dewetting process was investigated in this study with the cosine of the droplet contact angle $\left(\cos \boldsymbol{\theta}_{\mathrm{u}}\right)$ at diverse levels of the electric potential energy per unit area $\left(\boldsymbol{E}_{\mathrm{el}} / \boldsymbol{A}\right)$.

With respect to $\boldsymbol{E}_{\mathrm{el}} / \boldsymbol{A}$, a representative hysteresis behavior of $\cos \boldsymbol{\theta}_{\mathrm{u}}$ is shown in Figure 4.25 along an ascending voltage ramp (red, Stage I) followed by a descending voltage ramp (blue, Stage II and III).

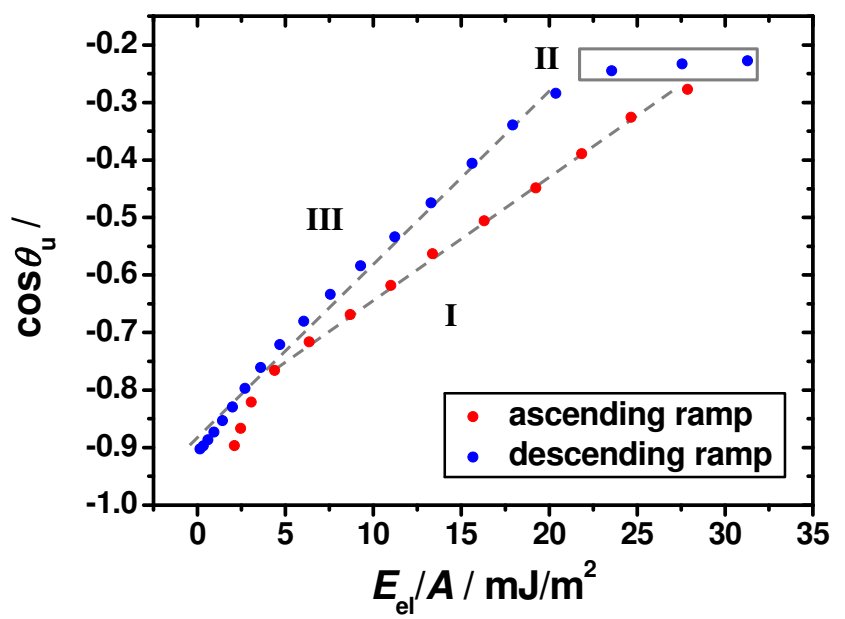

Figure 4.25. Cosine of the contact angle $\left(\cos \theta_{\mathrm{u}}\right)$ at diverse levels of the areal electrical potential energy $\left(E_{\mathrm{el}} / A\right)$ in the ascending (red) and the descending (blue) voltage ramp. Stage I: electrowetting; Stage II (enclosed square): inertia behavior in the switch from electrowetting to dewetting; Stage III: dewetting. Dashed lines: linear fitting lines $\left(\bar{R}^{2}=0.999\right.$ within Stage I, $\bar{R}^{2}=0.994$ at Stage III). ${ }^{\mathrm{XVI}}$

Starting from the low $\boldsymbol{E}_{\mathrm{el}} / \boldsymbol{A}$-level $\left(2 \mathrm{~mJ} / \mathrm{m}^{2}\right)$ corresponding to the voltage threshold $\left(\boldsymbol{U}_{\boldsymbol{t}}=2 \mathrm{~V}\right.$, Chapter 4.3.3.1), the $\cos \boldsymbol{\theta}_{\mathrm{u}}$ begins to increase and the electrowetting occurs at Stage I (Figure 4.25). The $\cos \boldsymbol{\theta}_{\mathrm{u}}$ shows firstly a strong rise with the $\boldsymbol{E}_{\mathrm{el}} / \boldsymbol{A}$ and subsequently an attenuated increase. This reveals a decrease in the efficiency of the electric potential energy in inducing electrowetting. The later increase reflects a linear correlation between the $\cos \boldsymbol{\theta}_{\mathbf{u}}$ and the $\boldsymbol{E}_{\mathrm{el}} / \boldsymbol{A}$. This is indicated by the adjusted coefficient of determination $\left(\overline{\boldsymbol{R}}^{2}=0.999\right)$ for the linear fitting (dashed line) in the $\boldsymbol{E}_{\mathrm{el}} / \boldsymbol{A}$-region from 4.3 to $27.9 \mathrm{~mJ} / \mathrm{m}^{2}$. This linear correlation coincides in principle with the Young-Lippmann equation (Eq. 2.2). This suggests that the equilibrium of interfacial interactions at the droplet-electrode-interface is rapidly achieved at each energy level in this region. This can be confirmed by the significantly shorter characteristic time $(\boldsymbol{\tau})$ in comparison with the period of a voltage step $(25 \mathrm{~ms})$ for this $\boldsymbol{E}_{\mathrm{el}} / \boldsymbol{A}$-region (Figure 4.27).

By switching from the ascending to the descending voltage ramp, the droplet shows an inertial behavior in Stage II (enclosed square in Figure 4.25). At this inertial stage, the droplet contact

$\mathrm{XVI}$ The omission of the data points in the measurement beginning is explained in Footnote $\mathrm{XV}$. 
angle declines merely slightly with the descending voltage ramp in spite of a significant amount of energy being withdrawn from the EWOD system. Moreover, a long response time is required for the very slight change of the contact angle at this stage (Figure 4.27). These results indicate a strong inertial behavior at this stage. The change of the droplet actuation from electrowetting (Stage I) to dewetting (Stage III) is related to the reversion of the flow impulse.

At Stage III, the droplet dewetting process can be described with an approximately linear correlation between $\cos \boldsymbol{\theta}_{\mathrm{u}}$ and $\boldsymbol{E}_{\mathrm{el}} / \boldsymbol{A}$, based on the linear fitting line (dashed line) with $\overline{\boldsymbol{R}}^{2}=0.994$. Furthermore, the low values of the corresponding $\boldsymbol{\tau}$ 's (Figure 4.27 ) indicate the equilibrium states being quickly reached and thus support the linear correlation principally reflecting the Young-Lippmann equation (Eq. 2.2). In comparison to the electrowetting behavior (Stage I), the dewetting behavior (Stage III) reveals a larger $\cos \boldsymbol{\theta}_{\mathbf{u}}$-alternation per unit $\boldsymbol{E}_{\text {el }} / \boldsymbol{A}$ above $4.4 \mathrm{~mJ} / \mathrm{m}^{2}$ according to the slopes of their fitting lines. However, below this $\boldsymbol{E}_{\mathrm{el}} / \boldsymbol{A}$-level, less $\cos \boldsymbol{\theta}_{\mathrm{u}}$-change is shown in the dewetting process than in the electrowetting process. In addition, the $\cos \boldsymbol{\theta}_{\mathrm{u}}$ correlates almost linearly with the $\boldsymbol{E}_{\mathrm{el}} / \boldsymbol{A}$ in the dewetting process. These results suggest that the conversion efficiency between the electric potential energy and the droplet deformation maintains in the dewetting process and decreases at the $\boldsymbol{E}_{\text {el }} / \boldsymbol{A}$-level of $4.4 \mathrm{~mJ} / \mathrm{m}^{2}$. Moreover, the oil layer deforms also more effectively in the electrowetting than in the dewetting process in the low $\boldsymbol{E}_{\mathrm{el}} / \boldsymbol{A}$-region (Figure 4.22, Chapter 4.3.2); however, this region ends at a much higher $\boldsymbol{E}_{\mathrm{el}} / \boldsymbol{A}$-boundary of $13 \mathrm{~mJ} / \mathrm{m}^{2}$. This means that the $\boldsymbol{E}_{\mathrm{el}} / \boldsymbol{A}$-range for more effective electrowetting is significantly narrower for the droplet deformation than that for the oil layer deformation. This indicates that more or stronger dissipation occurs related to the droplet deformation process than that to the oil layer deformation.

At the end of Stage III, the droplet returns to its resting state at zero Volt with a comparable contact angle to that at the beginning of the electrowetting, where $\boldsymbol{E}_{\mathrm{el}} / \boldsymbol{A}=2 \mathrm{~mJ} / \mathrm{m}^{2}$ (Stage I).

Comparing the electrowetting process (Stage I) with the dewetting process (Stage III), they cover almost the same $\cos \boldsymbol{\theta}_{\mathbf{u}}$-range; however, the electrowetting process occurs generally at higher $\boldsymbol{E}_{\mathrm{el}} / \boldsymbol{A}$-levels than the dewetting process. This means that the electrowetting process requires higher energy input than the energy withdrawn in the corresponding dewetting process.

In fact, the contact angle hysteresis between the ascending and the descending voltage ramps can be associated with four stages: the initial inertial stage prior to the electrowetting, the electrowetting process (Stage I), the inertial stage on alternating from electrowetting to dewetting (Stage II) and the dewetting process (Stage III). The initial inertial stage is not plotted in Figure 4.25 but is reflected by the voltage threshold (Chapter 4.3.3.1). The contact angle hysteresis can originate from the energy dissipation processe ${ }^{10,35}$ including overcoming the diverse frictions to actuate the droplet deformation, such as the fluid friction or the viscous effect in the droplet and in its surrounding oil as well as the surface friction responsible for the contact line pinning. 


\subsubsection{Contact Area Dynamics and Characteristic Time}

A representative time-resolved response of the droplet contact area to a voltage step stimulus is shown in Figure 4.26: the electrowetting process upon the voltage jump from $4.14 \mathrm{~V}$ to $4.42 \mathrm{~V}$. The contact area starts with the end-value reached at the previous voltage step. It increases gradually with the time and achieves almost a stabilized stage at the process end.

This contact area increase should follow the course of a first-order relaxation process ${ }^{49}$. The electrowetting process can be considered as the conversion of the droplet geometry from one with a smaller to a larger wetted area under the influence of an external factor, here the voltage. Hence, the dynamic electrowetting process is comparable to a first-order reaction and can be described as a first-order relaxation process with the exponential function $\left(\boldsymbol{A}=\boldsymbol{A}_{\boldsymbol{e}} \cdot\left(\mathbf{1}-\boldsymbol{e}^{-\frac{\boldsymbol{t}}{\tau}}\right)\right.$, Eq. 2.28).

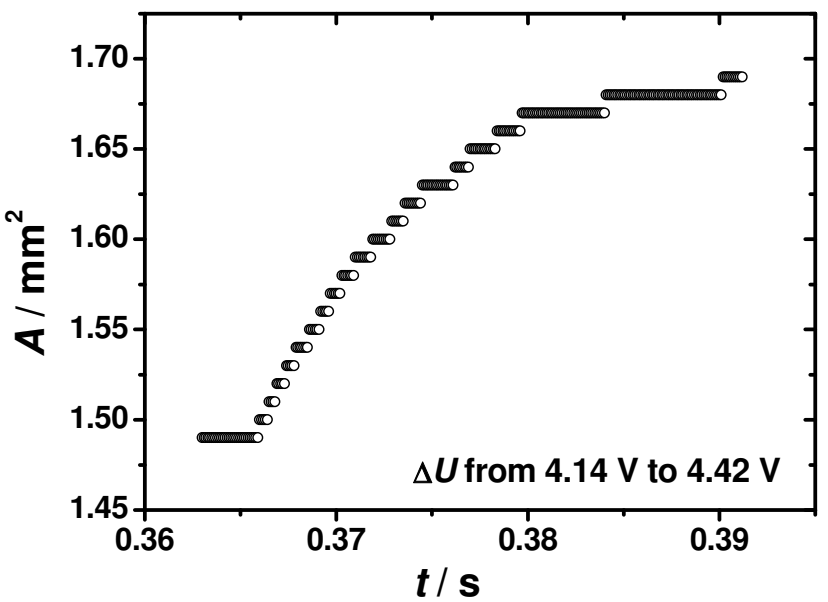

Figure 4.26. Time-resolved dynamic relaxation process of the contact area $(A)$ upon the voltage jump from $4.14 \mathrm{~V}$ to $4.42 \mathrm{~V}$.

Based on this approach, the contact area response was analyzed with Eq. 2.28 in case of an ascending voltage jump and with its negative expression, $\boldsymbol{A}=-\boldsymbol{A}_{\boldsymbol{e}} \cdot\left(\mathbf{1}-\boldsymbol{e}^{-\frac{\boldsymbol{t}}{\tau}}\right)$, in case of a descending voltage jump. Through curve fitting, the characteristic time $(\boldsymbol{\tau})$ was determined for the dynamic relaxation process of the contact area at all voltage steps of the staircase stimulus. For a first-order relaxation process, $\boldsymbol{\tau}$ can be easily used to assess the process velocity due to its inverse correlation to the process rate constant. With respect to the electric potential energy per unit area $\left(\boldsymbol{E}_{\mathrm{el}} / \boldsymbol{A}\right)$, the non-linear behavior of $\boldsymbol{\tau}$ is presented in Figure 4.27 for the ascending ramp in red and for the descending ramp in blue.

In both ascending and descending voltage ramps, the characteristic time behaves in a comparable way. With increasing $\boldsymbol{E}_{\mathrm{el}} / \boldsymbol{A}, \boldsymbol{\tau}$ shows a decline followed by a stabilized stage and an increase. This means that the droplet deformation process during the electrowetting firstly speeds up until an approximately constant rate is achieved and then slows down with the increasing supply of the electric potential energy per unit area. During the dewetting process, the droplet deformation velocity varies in an inverse matter. 


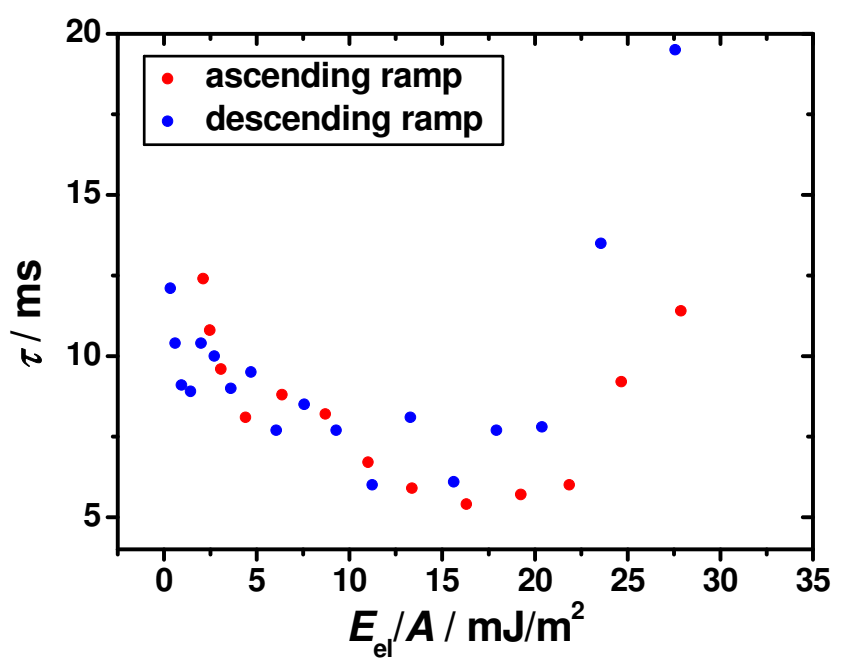

Figure 4.27. Non-linear correlation between the characteristic time $(\tau)$ and the electrical potential energy per unit area $\left(E_{\mathrm{el}} / A\right)$ for each voltage step in the ascending (red) and the descending (blue) voltage ramp. vVII $^{2}$

The $\boldsymbol{\tau}$-discrepancy between the electrowetting and the dewetting process is mostly significant at the high levels of $\boldsymbol{E}_{\mathrm{el}} / \boldsymbol{A}$. With the $\boldsymbol{E}_{\mathrm{el}} / \boldsymbol{A}$-decline, the $\boldsymbol{\tau}$-difference becomes less significant; comparable $\boldsymbol{\tau}$-values are found at the low levels of $\boldsymbol{E}_{\mathrm{el}} / \boldsymbol{A}$. This suggests the electrowetting process is faster than the dewetting process. The velocity difference diminishes with the reducing electrical potential energy per unit area. This hysteresis of $\boldsymbol{\tau}$ indicates the nonlinearity of the EWOD-system. It may originate from the difference between the "active" electrowetting process fed with the electric potential energy and the passive dewetting process, in which the droplet motion is more affected by the surface friction and viscous effect.

Moreover, the determined characteristic time is in the range between 5 and $20 \mathrm{~ms}$ (Figure 4.27). This range is comparable with the time scale ( 10 ms) corresponding to the boundary frequency $(\sim 100 \mathrm{~Hz})$ observed for this EWOD system, where the droplet reaches its hydrodynamic limitation to follow the excitation of an oscillating voltage signal. This coincident time scale could result from the common limitations in the droplet motion with a hydrodynamics-based origin, such as the viscous effect of the droplet and the oil.

In comparison to the period $(\boldsymbol{T}=0.1 \mathrm{~ms})$ of the applied EIS signal $(\boldsymbol{f}=10 \mathrm{kHz})$ and that $(\boldsymbol{T} \sim 1.4 \mathrm{~ms}$ ) of the optical measurement ( $\boldsymbol{v} \sim 700$ frames/s), the $\boldsymbol{\tau}$ of the droplet deformation process is significantly longer. This means that, within the observation window of each single EIS measurement and frame recording, the droplet maintains unchanged and can be described as "frozen" in time. Thus, the rapid EIS measurement and the optical imaging with a high-speed camera paves the way for a highly time-resolved characterization of the droplet deformation process.

\footnotetext{
$\mathrm{XVII}$ At the very low electric potential energy per unit area levels, the characteristic time could not be determined, since the contact area remains constant below the voltage threshold (Figure 4.24) due to the droplet inertia behavior.
} 


\subsubsection{Conclusions}

This section is devoted to the characterization of the non-linearity and the dynamics of the EWOD system (Figure 4.19 a) with a focus on the deformation processes during the electrowetting and dewetting in correlation with the alternation of the electric potential energy per unit area.

The investigation aim was successfully achieved by applying dynamic EIS associated with optical imaging. The staircase voltage signal of the dynamic EIS combines the voltage jumps for inducing electrowetting as well as dewetting with an integrated square function for online tracing of the system capacitance. Simultaneously, the optical imaging using a high-speed camera delivers the time-resolved droplet contact angle and contact area.

The non-linearity of EWOD was characterized by the hystereses in the deformation of the interfacial oil layer and in the droplet deformation with the stepwise voltage changes. With a view of the time-resolved deformation of the contact area at each voltage step, the determined characteristic time laid a foundation for analyzing the dynamics.

The electric potential energy per unit area $\left(\boldsymbol{E}_{\mathrm{el}} / \boldsymbol{A}\right)$ was determined with the MDS-capacitance per unit area $\left(\boldsymbol{C}_{\mathrm{MDS}} / \boldsymbol{A}\right)$ for each voltage offset in the staircase stimulus. It shows a nearly reversible behavior along the voltage staircase.

As key parameter for the oil layer deformation, the oil layer thickness was indirectly determined from the MDS-capacitance per unit area $\left(\boldsymbol{C}_{\text {MDS }} / \boldsymbol{A}\right)$ due to its nm-scale. The oil layer is indicated to flow out of the space between the droplet and the electrode during electrowetting and to flow back during dewetting. The outflow and the backflow of the oil layer is shown by the comparable efficiency of the conversion of the electric potential energy per unit area to the thickness alternation at the high $\boldsymbol{E}_{\mathrm{el}} / \boldsymbol{A}$-levels. At the low $\boldsymbol{E}_{\mathrm{el}} / \boldsymbol{A}$-levels, the electrowettingrelated outflow of the oil layer shows a higher energy-deformation-conversion efficiency than for the dewetting-related backflow. Thus, the deformation process of the oil layer is suggested to be reversible at high energy levels while hysteresis dominates at low energy levels.

Two characteristics of the droplet deformation were identified in this study: a quantified low voltage threshold to induce the electrowetting and the contact angle hysteresis between electrowetting and dewetting.

The voltage threshold was determined with the response of the contact angle $\left(\boldsymbol{U}_{t, \boldsymbol{\theta}}=1.93 \mathrm{~V} \pm 0.16 \mathrm{~V}\right)$ and with that of the contact area $\left(\boldsymbol{U}_{t, \boldsymbol{A}}=2.13 \mathrm{~V} \pm 0.17 \mathrm{~V}\right)$. The low values signify the low voltage demand for overcoming the droplet inertia to start electrowetting. The achievement of a low-voltage EWOD system in this doctoral research is confirmed with comparison with the literature values $(7.2-15 \mathrm{~V}){ }^{71,163-164}$. Moreover, the coincidence of the two values indicates the insignificance of the contact line pinning. Since this is a major source of dissipation ${ }^{10,50}$ in electrowetting and dewetting, this result suggests the high efficiency of the EWOD system. 
The contact angle hysteresis was studied with the $\cos \boldsymbol{\theta}_{\mathrm{u}}$-alternation with $\boldsymbol{E}_{\mathrm{el}} / \boldsymbol{A}$ for three stages along the voltage staircase upon the voltage threshold: the electrowetting, the inertia state upon the actuation direction switch and the dewetting. Among all stages, linear correlations between $\cos \boldsymbol{\theta}_{\mathrm{u}}$ and $\boldsymbol{E}_{\mathrm{el}} / \boldsymbol{A}$ can be observed in the dewetting stage and at high $\boldsymbol{E}_{\text {el }} / \boldsymbol{A}$-levels in the electrowetting stage. These linear correlations reflect in principle the YoungLippmann equation (Eq. 2.2). Their slope discrepancy indicates the different efficiency of the energy-deformation-conversion in electrowetting and dewetting. Furthermore, the droplet deforms in a significantly narrower $\boldsymbol{E}_{\text {el }} / \boldsymbol{A}$-range, where electrowetting is more effective than dewetting, in comparison with the oil layer does. This indicates more or stronger dissipation involved in the droplet deformation than that in the oil layer deformation.

The dynamics of the EWOD system was studied with the contact area response upon the voltage jumps in the staircase stimulus. Based on its principle as a first-order relaxation process, the deformation response was determined for its characteristic time $(\tau)$ to assess the deformation velocity. In both electrowetting and dewetting, the $\boldsymbol{\tau}$-alternation indicates the slow deformation at the high $\boldsymbol{E}_{\mathrm{el}} / \boldsymbol{A}$-levels and the quickly achieved balance of the interfacial forces in the EWOD system at the middle energy levels. The $\boldsymbol{\tau}$-hysteresis suggests a faster droplet deformation in the electrowetting than that in the dewetting. This further indicates dewetting to be more affected by dissipation than electrowetting. Moreover, the determined $\boldsymbol{\tau}$ between $5 \mathrm{~ms}$ and $20 \mathrm{~ms}$ is significantly longer than the measuring period of a single EIS and that of a single frame imaging. This ensured the successful time-resolved investigation of the droplet deformation.

The hysteresis between the electrowetting and the dewetting revealed by all the characterizations in this section can originate from the dissipation accompanying the deformation process of the oil layer and that of the droplet. The dissipation has been a major challenge in the fluid dynamics ${ }^{10,50}$ and may have diverse sources, such as fluid frictions or viscose effect and surface friction related contact line pinning. 


\section{SUMMARY}

The aims of this thesis are to optimize electrowetting on dielectrics (EWOD) on application of low voltages, to investigate the frequency dependence of EWOD in alternating voltage applications and to characterize the dynamics and non-linearity of EWOD and dewetting. The achievement of the low-voltage EWOD paves the way for its integration with electrical impedance spectroscopy (EIS) serving for the online electrical analysis of EWOD and dewetting.

First, a low-voltage EWOD (LV-EWOD) electrode was developed with the focus on optimizing the dielectric layers. To reduce EWOD operating voltages, three approaches originating from the Young-Lippmann equation were implemented by strategic selection of the dielectric materials and their fabrication methods. An optimized dielectric bilayer resulted from an anodic $\mathrm{Ta}_{2} \mathrm{O}_{5}$ initial layer coated with a self-assembled monolayer of octadecyltrichlorosilane (ODTS).

With an aqueous droplet of $\mu \mathrm{L}$ volume on the LV-EWOD electrode, the EWOD configuration uses oil as ambient medium due to its practical relevance for many EWOD applications. This results in a thin oil layer being entrapped between the droplet and the electrode as shown by Staicu and Mugele ${ }^{36}$. A multilayer dielectric stack (MDS) was postulated to describe the entire stack of dielectric multilayers in the EWOD configuration. It consists of the oil layer as a fluid dielectric layer and the solid dielectric electrode coatings.

To validate the layer quality and to adjust the fabrication process parameters, each layer of the MDS was characterized by five EWOD-related properties: hydrophobicity, surface friction, capacitance per unit area, layer thickness and relative permittivity.

The most appropriate anodizing conditions for $\mathrm{Ta}_{2} \mathrm{O}_{5}$ layers were studied at various anodization voltages $\left(\boldsymbol{U}_{\text {anod }}=20-50 \mathrm{~V}\right)$. An anodization voltage of $30 \mathrm{~V}$ was chosen based on the following outstanding features: smooth surface, homogeneous layer thickness $(\boldsymbol{d}=59.35 \mathrm{~nm} \pm 0.55 \mathrm{~nm})$, high reproducibility, reliability of capacitance per unit area $\left(\boldsymbol{C} / \boldsymbol{A}=3.93 \mathrm{nF} / \mathrm{mm}^{2} \pm 0.07 \mathrm{nF} / \mathrm{mm}^{2}\right)$ and high relative permittivity $\left(\boldsymbol{\varepsilon}_{\mathbf{d}}=26.3 \pm 0.5\right)$. For the anodization voltage range from $20 \mathrm{~V}$ to $50 \mathrm{~V}$, the layer growth coefficient was determined to be $(1.94 \pm 0.05) \mathrm{nm} / \mathrm{V}$. For anodization voltage from $30 \mathrm{~V}$ upwards, relative permittivity was shown to be independent of the anodization voltage. Based on the anodic $\mathrm{Ta}_{2} \mathrm{O}_{5}$ layer, a selfassembled ODTS monolayer $(\boldsymbol{d}=2.3 \mathrm{~nm} \pm 0.2 \mathrm{~nm})$ rendered the electrode surface hydrophobic. With a microscopic view of the interfacial interactions, this surface chemistry modification was indicated to contribute to the oil layer formation between the droplet and the electrode as a fluid dielectric layer. Due to its nanometer scale, the oil layer thickness ( $\boldsymbol{d}=15.2 \mathrm{~nm} \pm 7.2 \mathrm{~nm}$, without EWOD) is not easily accessible by direct experimental methods. In this study, the thickness of the oil layer was determined by measuring the layer capacitance indirectly and converting it to the thickness information using a physical model of the MDS. For the first time, this indirect methodology was applied for this purpose during EWOD. Moreover, by means of the indirect methodology, I determined the relative permittivity of the anodic $\mathrm{Ta}_{2} \mathrm{O}_{5}$ layer and that of the ODTS monolayer. Relative permittivity is a key material property for 
successful low-voltage EWOD. Its specification served initially for the validation of the fabrication conditions of the anodic $\mathrm{Ta}_{2} \mathrm{O}_{5}$ layer and later for the quality control of both dielectric solid layers. Based on the optimization of the dielectric layers, low-voltage EWOD was achieved in this study and confirmed by the low voltage threshold for the EWOD actuation in comparison with the literature values $(7.2-15 \mathrm{~V})^{71,163-164}$. The low voltage threshold was determined with contact angle $\left(\boldsymbol{U}_{\boldsymbol{t}, \boldsymbol{\theta}}=1.93 \mathrm{~V} \pm 0.16 \mathrm{~V}\right)$ and with contact area $\left(U_{t, A}=2.13 \mathrm{~V} \pm 0.17 \mathrm{~V}\right)$.

With the LV-EWOD electrode as the centerpiece, a low-voltage EWOD-EIS system was established in association with a high-speed optical imaging instrument. It enables the online investigation of EWOD by simultaneous EWOD excitation, impedimetric measurement and optical detection. Due to its modular construction, the EWOD-EIS system supports impedimetric measurements using diverse measuring techniques. In this thesis, frequencydomain EIS was applied for studying the frequency dependence of EWOD on application of alternating voltages; dynamic EIS was used for studying the non-linearity and dynamics of EWOD and dewetting. As the object under test in both studies, an aqueous electrolyte droplet on the MDS was modelled by an equivalent circuit. It represents the droplet as a resistor and the MDS as three capacitors connected in series. The validity of this circuit model was confirmed by the impedimetric response traced online by frequency-domain EIS.

Second, for the frequency dependence study, I adapted the basic Young-Lippmann equation to the alternating voltage application by using the effective value for the voltage drop across MDS and introducing a correlation coefficient $(\boldsymbol{\beta})$ as indicator for the EWOD efficiency. All three EWOD-related variables in the adapted Young-Lippmann equation were demonstrated to be frequency dependent: (i) The effective voltage drop across MDS shows a low-pass filter behavior with a critical frequency determined by the salt concentration in the droplet. (ii) The MDS-capacitance per unit area decreases with the frequency possibly due to the incomplete storage of the electric potential energy in the MDS due to the time limitation. At low frequencies, the increase of the capacitance per unit area with increasing voltage indicates a thickness decease of the interfacial oil layer during EWOD. (iii) The frequency- and voltage-dependent contact angle change indicates a voltage minimum and a frequency maximum for the EWOD actuation. Furthermore, EWOD dominates below a frequency threshold and is induced by partial conversion of the electric potential energy stored in MDS. In the low frequency region, the EWOD-related variables present a linear correlation with the adapted Young-Lippmann equation with $\boldsymbol{\beta}$ below one.

Third, the non-linearity and the dynamics of EWOD and dewetting was studied extensively for the first time using dynamic EIS in parallel to high-speed imaging. The dynamic EIS was configured with a staircase voltage signal in combination with a square wave function at each voltage step. Ascending and descending ramps induce EWOD and dewetting respectively, while the square wave signal serves as a high-frequency stimulus for the impedimetric measurement. By means of dynamic EIS, the electric potential energy stored in MDS per unit area $\left(\boldsymbol{E}_{\mathrm{el}} / \boldsymbol{A}\right)$ was determined in voltage resolution and in time resolution. This enabled the 
study of the non-linearity and dynamics during EWOD and dewetting with a new aspect of the electrical energy conversion in the deformation of the droplet and the interfacial oil layer.

The indirectly determined thickness of the interfacial oil layer was used to indicate its deformation. A thick oil layer was revealed to occupy the space between the droplet and the electrode before electrowetting and after dewetting. At the high $\boldsymbol{E}_{\mathrm{el}} / \boldsymbol{A}$-levels, its outflow with the progressing EWOD and its backflow with the dewetting were assessed to be reversible based on the comparable efficiencies of the energy conversion during deformation. At the low $\boldsymbol{E}_{\mathrm{el}} / \boldsymbol{A}$-levels, the oil layer deformation reveals a hysteresis with a more efficient energy conversion in the outflow during EWOD than that in the backflow during dewetting.

Moreover, the droplet deformation was evaluated by means of the contact angle and contact area to characterize the non-linearity of EWOD and dewetting. The quantified low voltage threshold with both parameters, as mentioned above, demonstrates that EWOD can be induced at a low voltage with the EWOD configuration developed in this study. The coincidence of the two voltage thresholds indicates the low dissipation relating to the contact line pinning and thus the high efficiency of this EWOD system. Furthermore, the contact angle hysteresis was evaluated with the $\cos \boldsymbol{\theta}_{\mathrm{u}}$-change with the $\boldsymbol{E}_{\mathrm{el}} / \boldsymbol{A}$. It was characterized by an inertia state corresponding to the switch from EWOD to dewetting and by the different efficiencies of the energy conversion to the droplet deformation in EWOD and dewetting. In comparison with the oil layer deformation, the droplet deformation was indicated to relate to more intensive energy dissipation.

To elucidate the dynamics of EWOD and dewetting, the characteristic time was determined for the droplet deformation process with a view of the contact area response upon the voltage jumps in the staircase stimulus. The characteristic time shows a hysteresis behavior indicating a faster deformation in EWOD than in dewetting. Moreover, the measuring period of a single EIS measurement and that of a single frame imaging is significantly shorter than the characteristic time $(\sim 5-20 \mathrm{~ms})$ of the droplet deformation process. This ensures the successful time-resolved investigation.

In this study, the low-voltage EWOD was successfully achieved and facilitates the diverse online investigations based on the simultaneous EWOD and EIS in association with highspeed imaging. In the context of common EWOD applications using alternating voltages, the EWOD principle was related to its frequency-dependent dielectric behavior. For the first time, the EWOD principle was extensively characterized for the non-linearity and the dynamic behavior of EWOD and dewetting using dynamic EIS in parallel with high-speed imaging. Moreover, the thickness of the interfacial oil layer was determined for an EWOD configuration using oil as surrounding medium as the common EWOD applications for the first time. The integration of low-voltage EWOD with EIS achieved in this study further indicates the promising future of EWOD-EIS-based actuator-sensor systems. 


\section{REFERENCES}

1. Guadarrama-Cetina, J.; Mongruel, A.; Medici, M.-G.; Baquero, E.; Parker, A. R.; Milimouk-Melnytchuk, I.; González-Viñas, W.; Beysens, D. Dew condensation on desert beetle skin. Eur. Phys. J. E 2014, 37, 109.

2. Zeng, X.; Qian, L.; Yuan, X.; Zhou, C.; Li, Z.; Cheng, J.; Xu, S.; Wang, S.; Pi, P.; Wen, X. Inspired by Stenocara Beetles: From Water Collection to High-Efficiency Water-in-Oil Emulsion Separation. ACS Nano 2017, 11, 760-769.

3. Lafuma, A.; Quéré, D. Superhydrophobic states. Nature Materials 2003, 2, 457.

4. Barthlott, W.; Neinhuis, C. Purity of the sacred lotus, or escape from contamination in biological surfaces. Planta 1997, 202, 1-8.

5. Tian, D.; Song, Y.; Jiang, L. Patterning of controllable surface wettability for printing techniques. Chem. Soc. Rev. 2013, 42, 5184-5209.

6. Midtdal, K.; Jelle, B. P. Self-cleaning glazing products: A state-of-the-art review and future research pathways. Sol. Energy Mater. Sol. Cells 2013, 109, 126-141.

7. Salta, M.; Wharton, J. A.; Stoodley, P.; Dennington, S. P.; Goodes, L. R.; Werwinski, S.; Mart, U.; Wood, R. J. K.; Stokes, K. R. Designing biomimetic antifouling surfaces. Philosophical Transactions of the Royal Society A: Mathematical, Physical and Engineering Sciences 2010, 368, 4729-4754.

8. Berge, B. Electrocapillarite et mouillage de films isolants par l'eau. Comptes Rendus- Academie des Sciences Paris Serie I/ 1993, 317, 157.

9. Lippmann, G. Relation entre les phénomènes électriques et capillaires. Ann. Chim. Phys 1875, 5, 494.

10. Bonn, D.; Eggers, J.; Indekeu, J.; Meunier, J.; Rolley, E. Wetting and spreading. Rev. Mod. Phys. 2009, 81, 739-805.

11. Zeng, J.; Korsmeyer, T. Principles of droplet electrohydrodynamics for lab-on-achip. Lab Chip 2004, 4, 265-277.

12. Hadwen, B.; Broder, G. R.; Morganti, D.; Jacobs, A.; Brown, C.; Hector, J. R.; Kubota, Y.; Morgan, H. Programmable large area digital microfluidic array with integrated droplet sensing for bioassays. Lab Chip 2012, 12, 3305-3313.

13. Fair, R. B. Digital microfluidics: is a true lab-on-a-chip possible? Microfluid. Nanofluid. 2007, 3, 245-281.

14. Wheeler, A. R. Putting Electrowetting to Work. Science 2008, 322, 539-540.

15. Li, L.; Liu, C.; Ren, H.; Wang, Q. H. Optical Switchable Electrowetting Lens. IEEE Photonics Technol. Lett. 2016, 28, 1505-1508. 
16. Berge, B.; Peseux, J. Variable focal lens controlled by an external voltage: An application of electrowetting. Eur. Phys. J. E 2000, 3, 159-163.

17. Xie, Y.; Sun, M.; Jin, M.; Zhou, G.; Shui, L. Two-phase microfluidic flow modeling in an electrowetting display microwell. Eur. Phys. J. E 2016, 39, 16 (5pp).

18. Hayes, R. A.; Feenstra, B. J. Video-speed electronic paper based on electrowetting. Nature 2003, 425, 383-385.

19. Abdelgawad, M.; Wheeler, A. R. The Digital Revolution: A New Paradigm for Microfluidics. Adv. Mater. 2009, 21, 920-925.

20. Cho, S. K.; Moon, H. Electrowetting on dielectric (EWOD): New tool for bio/micro fluids handling. Biochip Journal 2008, 2, 79-96.

21. Cho, S. K.; Moon, H.; Kim, C.-J. Creating, transporting, cutting, and merging liquid droplets by electrowetting-based actuation for digital microfluidic circuits. $J$. Microelectromech. Syst. 2003, 12, 70-80.

22. Abdelgawad, M.; Freire, S. L.; Yang, H.; Wheeler, A. R. All-terrain droplet actuation. Lab Chip 2008, 8, 672-7.

23. Rival, A.; Jary, D.; Delattre, C.; Fouillet, Y.; Castellan, G.; Bellemin-Comte, A.; Gidrol, X. An EWOD-based microfluidic chip for single-cell isolation, mRNA purification and subsequent multiplex qPCR. Lab Chip 2014, 14, 3739-3749.

24. Davoust, L.; da Cruz, C. A.; Theisen, J. On the use of AC electrowetting for biosensing based on dynamic contact angle. Sens. Actuators B Chem. 2016, 236, 849-857.

25. Seyrat, E.; Hayes, R. A. Amorphous fluoropolymers as insulators for reversible low-voltage electrowetting. J. Appl. Phys. 2001, 90, 1383-1386.

26. Paik, P.; Pamula, V. K.; Fair, R. B. Rapid droplet mixers for digital microfluidic systems. Lab Chip 2003, 3, 253-259.

27. Mibus, M.; Hu, X.; Knospe, C.; Reed, M. L.; Zangari, G. Failure Modes during Low-Voltage Electrowetting. ACS Appl. Mater. Interfaces 2016, 8, 15767-15777.

28. Berry, S.; Kedzierski, J.; Abedian, B. Irreversible Electrowetting on Thin Fluoropolymer Films. Langmuir 2007, 23, 12429-12435.

29. Dhindsa, M.; Heikenfeld, J.; Weekamp, W.; Kuiper, S. Electrowetting without Electrolysis on Self-Healing Dielectrics. Langmuir 2011, 27, 5665-5670.

30. Horowitz, P.; Hill, W. The Art of Electronics. 3. ed.; Cambridge University Press: United Kingdom, 2015.

31. Macdonald, J. R. Impedance Spectroscopy: Emphasizing Solid Materials and Systems. Wiley-Interscience: New York, Chichester, Brisbane, Toronto, Singapore, 1987. 
32. Barsoukov, E.; Macdonald, D. D. Impedance Spectroscopy Theory, Experiment, and Applications. 2 ed.; Wiley-Interscience: Hoboken, New Jersey, 2005.

33. Freire, S. L. S. Perspectives on digital microfluidics. Sens. Actuators A Phys. 2016, 250, 15-28.

34. Li, F.; Mugele, F. How to make sticky surfaces slippery: Contact angle hysteresis in electrowetting with alternating voltage. Appl. Phys. Lett. 2008, 92, 244108 (3pp).

35. Mugele, F.; Baret, J.-C. Electrowetting: from basics to applications. J. Phys.: Condens. Matter 2005, 17, R705-R774.

36. Staicu, A.; Mugele, F. Electrowetting-Induced Oil Film Entrapment and Instability. Phys. Rev. Lett. 2006, 97, 167801 (4pp).

37. Nelson, W. C.; Kim, C.-J. C. Droplet Actuation by Electrowetting-on-Dielectric (EWOD): A Review. J. Adhes. Sci. Technol. 2012, 26, 1747-1771.

38. Young, T. III. An essay on the cohesion of fluids. Philos. Trans. R. Soc. London 1805, 95, 65-87.

39. Butt, H.-J.; Graf, K.; Kappl, M. Physics and Chemistry of Interfaces. Wiley-VCH: Weinheim, 2003.

40. Frant, M. Funktionalisierung von Biowerkstoffen durch biomimetische Strukturen auf der Basis von Tetraetherlipiden - ein Antifoulingkonzept. Martin-LutherUniversität Halle-Wittenberg, 2008.

41. Zisman, W. A. Relation of the Equilibrium Contact Angle to Liquid and Solid Constitution. In Contact Angle, Wettability, and Adhesion, AMERICAN CHEMICAL SOCIETY: 1964; Chapter 1, pp 1-51.

42. Marmur, A. Solid-Surface Characterization by Wetting. Annual Review of Materials Research 2009, 39, 473-489.

43. Quéré, D. Wetting and Roughness. Annual Review of Materials Research 2008, 38, 71-99.

44. Jones, T. B.; Wang, K. L.; Yao, D. J. Frequency-Dependent Electromechanics of Aqueous Liquids: Electrowetting and Dielectrophoresis. Langmuir 2004, 20 , 2813-2818.

45. Jones, T. B.; Fowler, J. D.; Chang, Y. S.; Kim, C.-J. Frequency-Based Relationship of Electrowetting and Dielectrophoretic Liquid Microactuation. Langmuir 2003, 19, 7646-7651.

46. Jones, T. B. On the Relationship of Dielectrophoresis and Electrowetting. Langmuir 2002, 18, 4437-4443.

47. Melcher, J. R. Continnum Electromechanics. The MIT Press: Cambridge, Massachusetts, London, 1981. 
48. Tipler, P. A.; Mosca, G. Physics for Scientists and Engineers. 6. Ed. ed.; W. H. Freeman and Company: New York, 2006.

49. Atkins, P. W.; Paula, J. d. Physikalische Chemie. 4 ed.; Wiley-VCH Verlag: Weinheim, 2006.

50. Mugele, F. Fundamental challenges in electrowetting: from equilibrium shapes to contact angle saturation and drop dynamics. Soft Matter 2009, 5, 3377-3384.

51. Bockris, J. O. M.; Argade, S. D.; Gileadi, E. The determination of the potential of zero charge on solid metals. Electrochim. Acta 1969, 14, 1259-1283.

52. Macdonald, J. R.; Johnson, W. B. "Fundamentals of Impedance Spectroscopy" in Impedance Spectroscopy, Theory, Experiment, and Applications. 2. ed.; WileyInterscience: Hoboken, 2005.

53. Lasia, A. Electrochemical Impedance Spectroscopy and its Applications. Springer: New York, Heidelberg, Dordrecht, London, 2014.

54. Janshoff, A.; Steinem, C.; Wegener, J. "Non-invasive electrical sensor devices to monitor living cells online" in Thin film electrochemical sensors and biosensors: technology and performance. Springer Verlag: Heidelberg, 2004.

55. Grimnes, S. M., O Bioimpedance and Bioelectricity Basics. 3. ed.; Academic Press: Oxford, 2015.

56. Pliquett, U. Bioimpedance: A Review for Food Processing. Food Eng. Rev. 2010, 2, 74-94.

57. Ruffo, R.; Hong, S. S.; Chan, C. K.; Huggins, R. A.; Cui, Y. Impedance Analysis of Silicon Nanowire Lithium Ion Battery Anodes. J. Phys. Chem. C 2009, 113, 11390-11398.

58. Wang, Q.; Moser, J.-E.; Grätzel, M. Electrochemical Impedance Spectroscopic Analysis of Dye-Sensitized Solar Cells. J. Phys. Chem. B 2005, 109, 1494514953.

59. Cahill, B. P.; Land, R.; Nacke, T.; Min, M.; Beckmann, D. Contactless sensing of the conductivity of aqueous droplets in segmented flow. Sens. Actuators B Chem. 2011, 159, 286-293.

60. Lederer, T.; Clara, S.; Jakoby, B.; Hilber, W. Impedance spectroscopy on a digital microfluidic platform. Proc. SPIE 2011, 8066, 806625-806625.

61. Lederer, T.; Clara, S.; Jakoby, B.; Hilber, W. Integration of impedance spectroscopy sensors in a digital microfluidic platform. Microsystem Technologies 2012, 18, 1163-1180.

62. Hu, X.; Mibus, M.; Knospe, C. R.; Zangari, G.; Reed, M. L. Impedance spectroscopy and electrical modeling of electrowetting on dielectric devices. J. Micromech. Microeng. 2015, 25, 045020 (8pp). 
63. Hu, X.; Mibus, M.; Zangari, G.; Knospe, C.; Reed, M. L. Interrogation of Droplet Configuration During Electrowetting via Impedance Spectroscopy. J. Microelectromech. Syst. 2015, 24, 2092-2100.

64. Bard, A. J.; Faulkner, L. R. Electrochemical Methods: Fundamentals and Applications. 2 ed.; JOHN WILEY \& SONS, INC.: New York, Chichester, Weinheim, Brisbane, Singapore, Toronto, 2001.

65. Euler, L. "On Transcendental Quantities Which Arise from the Circle" in Introduction to the Analysis of the Infinite Springer Science: New York, 1988.

66. Orazem, M. E.; Tribollet, B. Electrochemical Impedance Spectroscopy. John Wiley \& Sons: Hoboken, New Jersey, 2008.

67. Foster, K. R.; Schwan, H. P. Dielectric properties of tissues and biological materials: A critical review. 1989; Vol. 17, p 25-104.

68. Pliquett, U. "Time Domain-Based Impedance Detection" in Capacitance Spectroscopy of Semiconductors. . Pan Stanford Publishing: 2018.

69. Teorell, T. Application of "Square Wave Analysis" to Bioelectric Studies1. Acta Physiol. Scand. 1946, 12, 235-254.

70. Huang, L. X.; Koo, B.; Kim, C. J. Sputtered Anodized $\mathrm{Ta}_{2} \mathrm{O}_{5}$ as the Dielectric Layer for Electrowetting-on-Dielectric. J. Microelectromech. Syst. 2013, 22, $253-$ 255.

71. Lin, Y.-Y.; Evans, R. D.; Welch, E.; Hsu, B.-N.; Madison, A. C.; Fair, R. B. Low voltage electrowetting-on-dielectric platform using multi-layer insulators. Sens. Actuators B Chem. 2010, 150, 465-470.

72. Li, Y.; Parkes, W.; Haworth, L. I.; Ross, A. W. S.; Stevenson, J. T. M.; Walton, A. J. Room-Temperature Fabrication of Anodic Tantalum Pentoxide for Low-Voltage Electrowetting on Dielectric (EWOD). J. Microelectromech. Syst. 2008, 17, 14811488.

73. Cahill, B. P.; Giannitsis, A. T.; Land, R.; Gastrock, G.; Pliquett, U.; Frense, D.; Min, M.; Beckmann, D. Reversible electrowetting on silanized silicon nitride. Sens. Actuators B Chem. 2010, 144, 380-386.

74. Kerrec, O.; Devilliers, D.; Groult, H.; Chemla, M. Dielectric properties of anodic oxide films on tantalum. Electrochim. Acta 1995, 40, 719-724.

75. Kim, Y.; Uosaki, K. Preparation of Tantalum Anodic Oxide Film in Citric Acid Solution - Evidence and Effects of Citrate Anion Incorporation. JOURNAL OF ELECTROCHEMICAL SCIENCE AND TECHNOLOGY 2014, 4, 163-170.

76. Young, L. The determination of the thickness, dielectric constant, and other properties of anodic oxide films on tantalum from the interference colours. Proceedings of the Royal Society of London. Series A. Mathematical and Physical Sciences 1958, 244, 41-53. 
77. Macagno, V.; Schultze, J. W. The growth and properties of thin oxide layers on tantalum electrodes. Journal of Electroanalytical Chemistry and Interfacial Electrochemistry 1984, 180, 157-170.

78. Boughaba, S.; Islam, M.; McCaffrey, J. P.; Sproule, G. I.; Graham, M. J. Ultrathin Ta2O5 films produced by large-area pulsed laser deposition. Thin Solid Films 2000, 371, 119-125.

79. Gala, F.; Zollo, G. Dielectric Properties of Self-Assembled Monolayer Coatings on a (111) Silicon Surface. J. Phys. Chem. C 2015, 119, 7264-7274.

80. Persano, A.; Quaranta, F.; Martucci, M. C.; Creti, P.; Siciliano, P.; Cola, A. Transport and charging mechanisms in Ta2O5 thin films for capacitive RF MEMS switches application. J. Appl. Phys. 2010, 107.

81. Jeliazova, Y.; Kayser, M.; Mildner, B.; Hassel, A. W.; Diesing, D. Temperature stability of thin anodic oxide films in metal/insulator/metal structures: A comparison between tantalum and aluminium oxide. Thin Solid Films 2006, 500, 330-335.

82. Christensen, C.; de Reus, R.; Bouwstra, S. Tantalum oxide thin films as protective coatings for sensors. J. Micromech. Microeng. 1999, 9, 113.

83. Chaneliere, C.; Autran, J. L.; Devine, R. A. B.; Balland, B. Tantalum pentoxide (Ta2O5) thin films for advanced dielectric applications. Materials Science and Engineering: R: Reports 1998, 22, 269-322.

84. Gebbert, A.; Alvarez-Icaza, M.; Stoecklein, W.; Schmid, R. D. Real-time monitoring of immunochemical interactions with a tantalum capacitance flowthrough cell. Anal. Chem. 1992, 64, 997-1003.

85. Ezhilvalavan, S.; Tseng, T. Y. Electrical properties of Ta2O5 thin films deposited on Ta. Appl. Phys. Lett. 1999, 74, 2477-2479.

86. Zhang, Y.; Ahn, P. B.; Fitzpatrick, D. C.; Heiner, A. D.; Poggie, R. A.; Brown, T. D. INTERFACIAL FRICTIONAL BEHAVIOR: CANCELLOUS BONE, CORTICAL BONE, AND A NOVEL POROUS TANTALUM BIOMATERIAL. J. Musculosketeletal Res. 1999, 03, 245-251.

87. Huang, N.-P.; Michel, R.; Voros, J.; Textor, M.; Hofer, R.; Rossi, A.; Elbert, D. L.; Hubbell, J. A.; Spencer, N. D. Poly(l-lysine)-g-poly(ethylene glycol) Layers on Metal Oxide Surfaces: Surface-Analytical Characterization and Resistance to Serum and Fibrinogen Adsorption. Langmuir 2001, 17, 489-498.

88. Clark, R. J. H.; Brown, D. The Chemistry of Vanadium, Niobium and Tantalum Pergamon Press: Oxford, New York, Toronto, Sydney, Paris, Braunschweig, 1973; Vol. 20.

89. Sloppy, J. D.; Lu, Z.; Dickey, E. C.; Macdonald, D. D. Growth mechanism of anodic tantalum pentoxide formed in phosphoric acid. Electrochim. Acta 2013, $87,82-91$. 
90. Wilcox, P. S.; Westwood, W. D. 'Anodic Oxidation of Tantalum. Can. J. Phys. 1971, 49, 1543-1548.

91. Chen, K.; Nielsen, M.; Rymaszewski, E. J.; Lu, T. M. Study of electron trapping in the amorphous tantalum oxide thin films prepared by d.c. magnetron reactive sputtering. Mater. Chem. Phys. 1997, 49, 42-45.

92. Kavanagh, Y.; Alam, M. J.; Cameron, D. C. The characteristics of thin film electroluminescent displays produced using sol-gel produced tantalum pentoxide and zinc sulfide. Thin Solid Films 2004, 447-448, 85-89.

93. Krasnov, A. N. Selection of dielectrics for alternating-current thin-film electroluminescent device. Thin Solid Films 1999, 347, 1-13.

94. Briehl, H. Chemie der Werkstoffe. 3 ed.; Springer: 2014.

95. Ueno, K.; Abe, S.; Onoki, R.; Saiki, K. Anodization of electrolytically polished Ta surfaces for enhancement of carrier injection into organic field-effect transistors. J. Appl. Phys. 2005, 98, 114503.

96. Albella, J. M.; Montero, I.; Sánchez, O.; Martínez - Duart, J. M. Theoretical Approach for the Constant Voltage Stage in Anodic Oxidation. J. Electrochem. Soc. 1986, 133, 876-879.

97. Wang, Y.; Lieberman, M. Growth of Ultrasmooth Octadecyltrichlorosilane SelfAssembled Monolayers on SiO2. Langmuir 2003, 19, 1159-1167.

98. Chinappi, M.; Casciola, C. M. Intrinsic slip on hydrophobic self-assembled monolayer coatings. Phys. Fluids 2010, 22, 042003.

99. Lane, J. M. D.; Chandross, M.; Lorenz, C. D.; Stevens, M. J.; Grest, G. S. Water Penetration of Damaged Self-Assembled Monolayers. Langmuir 2008, 24, 57345739.

100. Vuillaume, D.; Boulas, C.; Collet, J.; Davidovits, J. V.; Rondelez, F. Organic insulating films of nanometer thicknesses. Appl. Phys. Lett. 1996, 69, 1646-1648.

101. Zhang, H.; Guo, X.; Hui, J.; Hu, S.; Xu, W.; Zhu, D. Interface Engineering of Semiconductor/Dielectric Heterojunctions toward Functional Organic Thin-Film Transistors. Nano Lett. 2011, 11, 4939-4946.

102. Saliterman, S. Fundamentals of BioMEMS and Medical Microdevices. SPIE press, Wiley Interscience: Bellingham, Washington USA, 2006.

103. Onclin, S.; Ravoo, B. J.; Reinhoudt, D. N. Engineering Silicon Oxide Surfaces Using Self - Assembled Monolayers. Angew. Chem. Int. Ed. 2005, 44, 6282-6304.

104. Ulman, A. Formation and Structure of Self-Assembled Monolayers. Chem. Rev. 1996, 96, 1533-1554.

105. Schwartz, D. K. MECHANISMS AND KINETICS OF SELF-ASSEMBLED MONOLAYER FORMATION. Annu. Rev. Phys. Chem. 2001, 52, 107-137. 
106. Shapiro, B.; Moon, H.; Garrell, R. L.; Kim, C.-J. C. Equilibrium behavior of sessile drops under surface tension, applied external fields, and material variations. $J$. Appl. Phys. 2003, 93, 5794-5811.

107. Tietze, U.; Schenk, C. Halbleiter-Schaltungstechnik. 11 ed.; Spinger: Berlin, Heidelberg, 1999.

108. Hassel, A. W.; Diesing, D. Breakdown of ultrathin anodic valve metal oxide films in metal-insulator-metal-contacts compared with metal-insulator-electrolyte contacts. Thin Solid Films 2002, 414, 296-303.

109. Mott-Smith, H. M. Histroy of "Plasmas". Nature 1971, 233, 219.

110. Tonks, L. The Birth of "Plasma". Am. J. Phys. 1967, 35, 857-858.

111. Goldston, R. J.; Rutherford, P. H. Introduction to Plasma Physics. CRC Press: 1995.

112. Kong, M. G.; Kroesen, G.; Morfill, G.; Nosenko, T.; Shimizu, T.; Dijk, J. v.; Zimmermann, J. L. Plasma medicine: an introductory review. New Journal of Physics 2009, 11, 115012.

113. Hidding, B.; Manahan, G. G.; Karger, O.; Knetsch, A.; Wittig, G.; Jaroszynski, D. A.; Sheng, Z. M.; Xi, Y.; Deng, A.; Rosenzweig, J. B.; Andonian, G.; Murokh, A.; Pretzler, G.; Bruhwiler, D. L.; Smith, J. Ultrahigh brightness bunches from hybrid plasma accelerators as drivers of 5th generation light sources. Journal of Physics B: Atomic, Molecular and Optical Physics 2014, 47, 234010.

114. Gerlach, G.; Dötzel, W. Einführung in die Mikrosystemtechnik: ein Kursbuch für Studierende. Carl Hanser Verlag GmbH \& Co. KG 2006; p 385.

115. Menz, W.; Mohr, J.; Paul, O. Mikrosystemtechnik für Ingenieure. 3 ed.; WileyVCH: 2005.

116. De Palma, R.; Laureyn, W.; Frederix, F.; Bonroy, K.; Pireaux, J.-J.; Borghs, G.; Maes, G. Formation of Dense Self-assembled Monolayers of (nDecyl)trichlorosilanes on Ta/Ta2O5. Langmuir 2007, 23, 443-451.

117. Coates, M.; Connell, D. W.; Barron, D. M. Aqueous solubility and octan-1-ol-water partition coefficients of aliphatic hydrocarbons. Environ. Sci. Technol. 1985, 19, 628-632.

118. BioLogic Science Instruments EC-Lab Software: Techniques and Applications (Version 10.38). 2014.

119. BioLogic Science Instruments EC-Lab Software: User's Manual. 2014.

120. Hamann, C. H.; Vielstich, W. Elektrochemie. 4 ed.; Wiley-VCH Verlag: Weinheim, 2005.

121. Wang, J. Analytical Electrochemistry. VCH: New York, Weinheim, Cambridge, 1994. 
122. Fujiwara, H. Spectroscopic Ellipsometry: Principles and Applications. John Wiley \& Sons: England, 2007.

123. Tompkins, H. G.; McGahan, W. A. Spectroscopic Ellipsometry and Reflectometry: A User's Guide. Wiley: 1999.

124. Azzam, R. M. A.; Bashara, N. M. Ellipsometry and Polarized Light. North Holland: Amsterdam, 1977.

125. Accurion Nanofilm EP3 Operating Instructions (Software version: 2.3x). 2010.

126. Accurion EP4 Model Manual (for Versions 1.0.1+). 2009.

127. Muth, D. G. Ellipsometer Study of Anodic Oxides Formed on Sputtered Tantalum and Tantalum-Aluminum Alloy Films. J. Vac. Sci. Technol. 1969, 6, 749-752.

128. Lide, D. R. CRC Handbook of Chemistry and Physics 88TH Edition 2007-2008. CRC Press, Taylor \& Francis: Boca Raton, 2007.

129. Winter, R.; Noll, F.; C., C. Methoden der Biophysikaliscen Chemie. 2 ed.; Vieweg + Teubner Verlag: 2011.

130. Haugstad, G. Atomic Force Microscopy: Understanding Basic Modes and Advanced Applications. John Wiley \& Sons: 2012.

131. JPK instruments NanoWizard Series User manual: SPM Software Release 6.0. 2016.

132. Butt, H. J.; Franz, V. Water in Biomaterials Surface Science: 9. Atomic Force Microscope Analysis of Water-Mediated Interfacial Interactions. Wiley: 2001.

133. JPK instruments JPK DP Data Processing Software Manual: Version 6.0. 2016.

134. dataphysics Betriebsanleitung DataPhysics OCA-Serie. 2011.

135. Eral, H. B.; 't Mannetje, D. J. C. M.; Oh, J. M. Contact angle hysteresis: a review of fundamentals and applications. Colloid. Polym. Sci. 2013, 291, 247-260.

136. Technologies, A. V. AVT Pike: Technical Manual V4.0.0. 2008.

137. IBA Software programs applied in the doctoral research by Yingjia Li. https://www.iba-heiligenstadt.de/index.php?id=478\&L=0.

138. Hecht, E. Optics. Pearson Education: 2002.

139. Stavenga, D. G. Thin Film and Multilayer Optics Cause Structural Colors of Many Insects and Birds. Materials Today: Proceedings 2014, 1, 109-121.

140. Gauglitz, G.; Brecht, A.; Kraus, G.; Mahm, W. Chemical and biochemical sensors based on interferometry at thin (multi-) layers. Sens. Actuators B Chem. 1993, 11, 21-27. 
141. Yu, L.; Xu, H.; Monro, T. M.; Lancaster, D. G.; Xie, Y.; Zeng, H.; Chen, G. Y.; Liu, $X$. Ultrafast colorimetric humidity-sensitive polyelectrolyte coating for touchless control. Materials Horizons 2017, 4, 72-82.

142. Chi, H.; Liu, Y. J.; Wang, F.; He, C. Highly Sensitive and Fast Response Colorimetric Humidity Sensors Based on Graphene Oxides Film. ACS Appl. Mater. Interfaces 2015, 7, 19882-19886.

143. Everitt, B. The Cambridge Dictionary of Statistics. Cambridge University Press: Cambridge, UK New York, 1998.

144. Chen, X.; Xu, Z.; Wu, K.; Zhang, S.; Li, H.; Meng, Y.; Wang, Z.; Li, L.; Ma, X. Facile Peeling Method as a Post-Remedy Strategy for Producing an Ultrasmooth Self-Assembled Monolayer for High-Performance Organic Transistors. Langmuir 2016, 32, 9492-9500.

145. Li, Y.-J.; Cahill, B. P. Frequency Dependence of Low-Voltage Electrowetting Investigated by Impedance Spectroscopy. Langmuir 2017, 33, 13139-13147.

146. McGovern, M. E.; Kallury, K. M. R.; Thompson, M. Role of Solvent on the Silanization of Glass with Octadecyltrichlorosilane. Langmuir 1994, 10, 36073614.

147. Wasserman, S. R.; Tao, Y. T.; Whitesides, G. M. Structure and reactivity of alkylsiloxane monolayers formed by reaction of alkyltrichlorosilanes on silicon substrates. Langmuir 1989, 5, 1074-1087.

148. Bush, B. G.; DelRio, F. W.; Opatkiewicz, J.; Maboudian, R.; Carraro, C. Effect of Formation Temperature and Roughness on Surface Potential of Octadecyltrichlorosilane Self-Assembled Monolayer on Silicon Surfaces. The Journal of Physical Chemistry A 2007, 111, 12339-12343.

149. Bartell, L. S. On the Length of the Carbon-Carbon Single Bond1. J. Am. Chem. Soc. 1959, 81, 3497-3498.

150. Breitmaier, E.; Jung, G. Organische Chemie: Grundlagen, Stoffklassen, Reaktionen, Konzepte, Molekülstruktur. 4. ed.; Georg Thieme Verlag: Stuttgart, New York, 2001.

151. Pit, A.; Duits, M.; Mugele, F. Droplet Manipulations in Two Phase Flow Microfluidics. Micromachines 2015, 6, 1455.

152. Lohrengel, M. M. Thin anodic oxide layers on aluminium and other valve metals: high field regime. Materials Science and Engineering: $R$ : Reports 1993, 11, 243294.

153. Haynes, W. M. CRC Handbook of Chemistry and Physics, 93rd Edition 20122013. CRC Press, Taylor \& Francis: Boca Raton, London, New York, 2012.

154. Abbe, E. Beiträge zur Theorie des Mikroskops und der mikroskopischen Wahrnehmung. Archiv für mikroskopische Anatomie 1873, 9, 413-418. 
155. Hell, S. W. Nanoscopy with Focused Light (Nobel Lecture). Angew. Chem. Int. Ed. 2015, 54, 8054-8066.

156. Woods, D. A.; Bain, C. D. Total internal reflection Raman spectroscopy. Analyst 2012, 137, 35-48.

157. Woods, D. A.; Bain, C. D. Total internal reflection spectroscopy for studying soft matter. Soft Matter 2014, 10, 1071-1096.

158. Pit, A. M.; Ruiter, R. d.; Kumar, A.; Wijnperlé, D.; Duits, M. H. G.; Mugele, F. Highthroughput sorting of drops in microfluidic chips using electric capacitance. Biomicrofluidics 2015, 9, 044116.

159. Xie, Y.; Sun, M.; Jin, M.; Zhou, G.; Shui, L. Two-phase microfluidic flow modeling in an electrowetting display microwell. Eur. Phys. J. E 2016, 39, 16.

160. Mathematikkommission;, D.; Physikkommission, D. Formeln und Tafel (Mathematik-Statistik-Physik). 2. ed.; Orell Füssli Verlag Zürich: 1981.

161. Gau, H.; Herminghaus, S.; Lenz, P.; Lipowsky, R. Liquid Morphologies on Structured Surfaces: From Microchannels to Microchips. Science 1999, 283, 4649.

162. Quilliet, C.; Berge, B. Investigation of effective interface potentials by electrowetting. EPL (Europhysics Letters) 2002, 60, 99.

163. Khodayari, M.; Carballo, J.; Crane, N. B. A material system for reliable low voltage anodic electrowetting. Mater. Lett. 2012, 69, 96-99.

164. Moon, H.; Cho, S. K.; Garrell, R. L.; Kim, C.-J. C. Low voltage electrowetting-ondielectric. J. Appl. Phys. 2002, 92, 4080-4087.

165. Ren, H.; Fair, R. B.; Pollack, M. G.; Shaughnessy, E. J. Dynamics of electrowetting droplet transport. Sens. Actuators B Chem. 2002, 87, 201-206.

166. Wang, K. L.; Jones, T. B. Electrowetting Dynamics of Microfluidic Actuation. Langmuir 2005, 21, 4211-4217. 


\section{APPENDIX}

\subsection{Equation Derivations}

\subsubsection{Equation for Assessment of Dynamic Relaxation Process}

The dynamic process of droplet deformation is analyzed based on the change of the contact area between the droplet and the EWOD electrode upon each voltage jump within the staircase voltage stimulus. For a voltage step in the ascending ramp, the contact area increase is illustrated as a typical relaxation process as below.

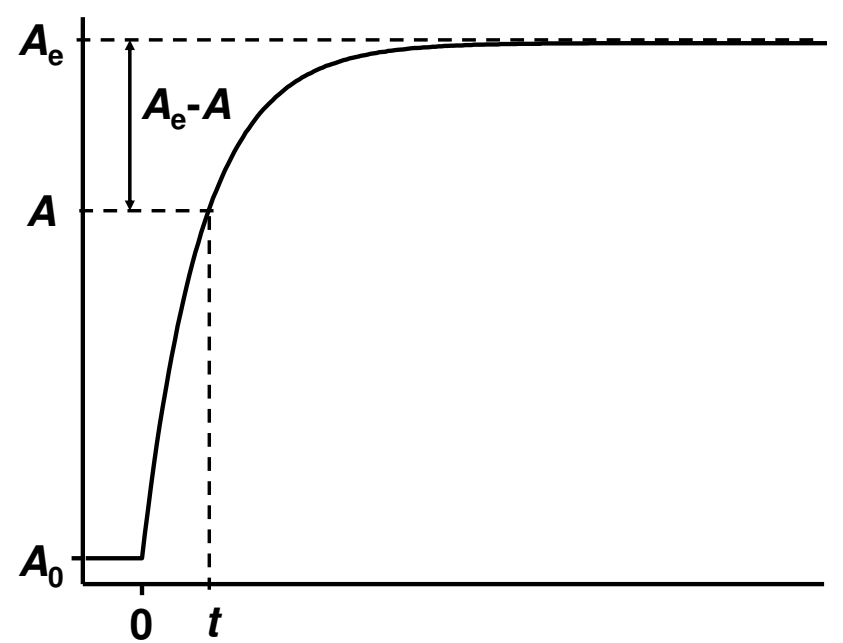

Figure A.1. Increase of the droplet contact area to its equilibrium end state upon a voltage step applied for a sufficient long period.

The difference between the maximal area value at the equilibrium end state $\left(\boldsymbol{A}_{\boldsymbol{e}}\right)$ and the contact area $(\boldsymbol{A})$ at time $\boldsymbol{t}$ is $\boldsymbol{A}_{\boldsymbol{e}}-\boldsymbol{A}$. The decrease of this area difference as a function of time can be regarded as a first-order reaction and is given by:

$$
\frac{d A}{d t}=\left(A_{e}-A\right) \cdot k \cdot t
$$

with $\boldsymbol{k}$ standing for the first order reaction rate.

The integrated form of Eq. A.1 is derived as below:

Eq. A. 1 is rearranged to

$$
\frac{d A}{A_{e}-A}=k \cdot t \cdot d t
$$

Eq. A.2 should be integrated over the time interval from zero to $t$ and over the area interval from the initial area $\left(\boldsymbol{A}_{\mathbf{0}}\right)$ to $\boldsymbol{A}$. The integral can be written as, 


$$
\int_{A_{0}}^{A} \frac{d A}{A_{e}-A}=\int_{0}^{t} k \cdot t \cdot d t
$$

which can be transformed using the substitution, $A_{e}-A=Z \Rightarrow \frac{d Z}{d A}=-\mathbf{1} \Rightarrow d \boldsymbol{A}=-\boldsymbol{d Z}$, to

$$
\int_{A_{0}}^{A}-\frac{d Z}{Z}=\int_{0}^{t} k \cdot t \cdot d t
$$

The integral results in

$$
-\left.\ln Z\right|_{A_{0}} ^{A}=\left.k \cdot t\right|_{0} ^{t}
$$

By replacing $\boldsymbol{Z}$ with $\boldsymbol{A}_{\boldsymbol{e}}-\boldsymbol{A}$, the calculation is continued as,

$$
\begin{gathered}
-\left.\ln \left(A_{e}-A\right)\right|_{A_{0}} ^{A}=\left.k \cdot t\right|_{0} ^{t} \\
-\ln \left(A_{e}-A\right)+\ln \left(A_{e}-A_{i}\right)=k \cdot t \\
\ln \frac{\left(A_{e}-A_{0}\right)}{\left(A_{e}-A\right)}=k \cdot t
\end{gathered}
$$

with $A_{0}=0$,

$$
\begin{gathered}
\ln \frac{A_{e}}{A_{e}-A}=k \cdot t \\
\frac{A_{e}}{A_{e}-A}=e^{k \cdot t} \\
A_{e} \cdot e^{-k \cdot t}=A_{e}-A \\
A=A_{e}\left(1-e^{-k \cdot t}\right)
\end{gathered}
$$

Since the characteristic time $(\boldsymbol{\tau})$ is equal to the reciprocal value of the reaction rate,

$$
A=A_{e}\left(1-e^{-\frac{t}{\tau}}\right)
$$

Thus, the Eq. 2.28 in Chapter 2.2.2.2 is obtained. 


\subsubsection{Root-Mean-Square Value of the Positive Alternating Voltages}

For the investigation of the influence of frequency on the EWOD effect, an alternating voltage stimulus in the positive region is applied to the EWOD system. This voltage stimulus is a sinusoidal signal with a direct voltage offset $\left(\boldsymbol{A}_{\mathbf{0}}\right)$ that equals the amplitude $\left(\boldsymbol{A}_{1}\right)$, the half value of the peak-to-peak voltage $\left(\boldsymbol{U}_{\mathbf{p p} / \mathbf{2}}\right)$. The voltage stimulus can be expressed as:

$$
U(t)=A_{0}+A_{1} \cdot \sin \omega t
$$

Here, $\boldsymbol{\omega}$ is the angular frequency of the sinusoidal wave function and $\boldsymbol{t}$ is time.

Based on $\boldsymbol{A}_{\mathbf{0}}=\boldsymbol{A}_{\mathbf{1}}=\boldsymbol{U}_{\mathrm{pp} / \mathbf{2}}$, Eq. A.14 can be transformed to:

$$
U(t)=U_{p p / 2} \cdot(1+\sin \omega t)
$$

The effective value, the RMS value, of the applied alternating voltage $\left(\boldsymbol{U}_{\mathbf{R M S}}\right)$ can be calculated according to its definition as below:

$$
\begin{aligned}
U_{R M S}=\sqrt{\left(\overline{U^{2}}\right)} & =\sqrt{\frac{1}{T} \cdot \int_{0}^{T} U^{2} d t} \\
= & \sqrt{\frac{1}{T} \cdot \int_{0}^{T}\left[U_{p p / 2} \cdot(1+\sin \omega t)\right]^{2} \cdot d t}
\end{aligned}
$$

with $\boldsymbol{T}$ standing for the period of the sinusoidal wave function.

Based on the following derivation ${ }^{\mathrm{XVII}}$ of $\boldsymbol{U}_{\mathrm{RMS}}^{2}$,

$$
\begin{gathered}
U_{\mathrm{RMS}}^{2}=\frac{1}{T} \cdot \int_{0}^{T}\left[U_{\mathrm{pp} / 2} \cdot(1+\sin \omega t)\right]^{2} \cdot d t \\
=\frac{1}{T} \cdot \int_{0}^{T} U_{\mathrm{pp} / 2}^{2} \cdot\left[1+2 \sin \omega t+(\sin \omega t)^{2}\right] \cdot d t \\
=\frac{1}{T} \cdot U_{\mathrm{pp} / 2}^{2} \cdot\left[\int_{0}^{T} 1 \cdot d t+\int_{0}^{T} 2 \sin \omega t \cdot d t+\int_{0}^{T}\left(\frac{1}{2}-\frac{\cos 2 \omega t}{2}\right) \cdot d t\right]
\end{gathered}
$$

\footnotetext{
XVIII The derivation is based on Nastase, A. S., How to Derive the RMS Value of a Sine Wave with a DC Offset, URL: http://masteringelectronicsdesign.com/how-to-derive-the-rms-value-of-a-sine-wave-witha-dc-offset/ [12.6.2017].
} 


$$
=\frac{1}{T} \cdot U_{\mathrm{pp} / 2}^{2} \cdot\left[\left.t\right|_{0} ^{T}+\left.\left(\frac{2}{\omega} \cdot \cos \omega t\right)\right|_{0} ^{T}+\left.\frac{t}{2}\right|_{0} ^{T}-\left.\frac{1}{4 \omega} \sin 2 \omega t\right|_{0} ^{T}\right] \quad \text { A.17. }
$$

Since $\boldsymbol{\omega}=\frac{2 \pi}{T}$

$$
\begin{gathered}
U_{\mathrm{RMS}}^{2}=\frac{1}{T} \cdot U_{\mathrm{pp} / 2}^{2} \cdot\left[T+\frac{T}{\pi}(\cos 2 \pi-\cos 0)+\frac{T}{2}-\frac{T}{8 \pi}(\sin 4 \pi-\sin 0)\right] \\
=\frac{3}{2} U_{\mathrm{pp} / 2}^{2}
\end{gathered}
$$

Therefore, the RMS-value of the applied positive sinusoidal signal is:

$$
U_{\mathrm{RMS}}=\sqrt{3 / 2} \cdot U_{\mathrm{pp} / 2}
$$




\subsection{List of Figures}

Figure 2.1. Electrowetting on dielectric (EWOD) effect demonstrated by a conductive droplet (dark blue) resting on a dielectric solid layer (bright blue) associated with an electrode layer (yellow) on a substrate (gray). (a) Under zero voltage: resting or dewetted state of the droplet with the static contact angle, $\boldsymbol{\theta}_{\mathbf{0}}$. (b) Under voltage: wetting state of the droplet with the voltage-dependent contact angle, $\boldsymbol{\theta}_{\mathbf{u}}$.

Figure 2.2. Equilibrium state of the three interfacial tensions on the triple-phase contact line. $\boldsymbol{\theta}$ : Static contact angle. $\boldsymbol{\gamma}_{\mathbf{s f}}$ : Solid-fluid interfacial tension. $\boldsymbol{\gamma}_{\mathbf{s l}}$ : Solid-liquid interfacial tension. $\gamma_{\text {lf }}$ : Liquid-fluid interfacial tension

Figure 2.3. Measurement principle of EIS in frequency domain. $U$ : sinusoidal voltage stimulus (red), l: current response (blue), $\phi$ : phase shift between the voltage and current signals.

Figure 2.4. Series connection of a resistor and a capacitor.

Figure 2.5. Square wave as a series of alternating positive (red) and negative (blue) voltage steps.

Figure 2.6. Basic principle of EIS in time domain demonstrated for a series $R C$ circuit. (a) $\mathrm{A}$ positive voltage step as electrical perturbation. (b) Current response with the time constant $(\boldsymbol{\tau})$.

Figure 2.7. Dynamic EIS signal for investigation into the dynamic behavior of EWOD effect. Main panel: Staircase voltage signal for the EWOD excitation. Inset (enlargement of the green marked region): Square signal superposed in each DC voltage step for EIS measurement in time domain.

Figure 2.8. Overview of the development process and the diverse aspects concerning the online electrical investigation of EWOD effect.

Figure 2.9. General layer structure of EWOD electrodes.

Figure 2.10. Principle of the self-assembled monolayer formation.

Figure 2.11. Optical image of a $3 \mu \mathrm{L}$ aqueous droplet on the EWOD electrode in oil with an inserted counter electrode and schematic abstract of the system under investigation.

Figure 2.12. (a) Equivalent circuit model for the system under investigation and (b) simplified equivalent circuit model. $\boldsymbol{R}_{\text {drop }}$ : droplet resistance; $\boldsymbol{C}_{\mathbf{d r o p}}$ : droplet capacitance; $\boldsymbol{R}_{\mathrm{MDS}}$ : total resistance of the multilayer dielectric stack; $\boldsymbol{C}_{\mathbf{o i l}}, \boldsymbol{C}_{\text {silane }}$ and $\boldsymbol{C}_{\mathrm{Ta}_{2} \mathrm{O}_{5}}$ : separated capacitances of the oil film, the silane layer and the $\mathrm{Ta}_{2} \mathrm{O}_{5}$-layer respectively; $\boldsymbol{C}_{\mathrm{MDS}}$ : total capacitance of the multilayer dielectric stack, composed of $C_{\text {oil }}, C_{\text {silane }}$ and $C_{\mathrm{Ta}_{2} \mathrm{O}_{5}}$ in series.

Figure 2.13. Circuit of a low-pass filter consisting of a resistor and a capacitor in series. $V_{\text {in: }}$ Signal input. $V_{\text {out }}$ Signal output. .23

Figure 3.1. Overview scheme of the fabrication processes for the low-voltage EWOD electrode.

Figure 3.2. Diced substrates covered with photo resist (left). One specimen after cleaning (right). .28 
Figure 3.3. PTFE sample holder with four specimens for the cleaning procedures.

Figure 3.4. Scheme of an electrochemical cell configured with two electrodes for potentiostatic anodization and an ammeter for current measurement. CE: counter electrode, WE: working electrode.

Figure 3.5. Anodization cell (left) facilitates a defined round area of the tantalum as the working electrode (WE). Separated anodization cell (right): two seal rings, housing bottom, top housing nut and middle part with a central aperture and a wired spring pin. .30

Figure 3.6. Logarithmic plot of current density over time for a representative anodization process of tantalum at $30 \mathrm{~V}$ in $0.1 \mathrm{M}$ citric acid for $90 \mathrm{~min}$.

Figure 3.7. Chemical structure of octadecyltrichlorosilane (ODTS).

Figure 3.8. Scheme of an electrochemical cell configured with three electrodes in a bulk solution and a potentiostat for the electrical impedance spectroscopy. CE: counter electrode, RE: reference electrode, WE: working electrode.

Figure 3.9. (a) Bode plot (black dots) of impedance modulus (left axis) and phase shift (right axis) against frequency for a $\mathrm{Ta}_{2} \mathrm{O}_{5}$ layer anodized at $50 \mathrm{~V}$ measured with PEIS. The fitted curves (red) based on (b) Equivalent circuit model for the tantalum oxide layer and the measurement solution. $R_{1}$ : Resistance of the measurement solution, $C_{2}, R_{2}$ : Capacitance and resistance of tantalum oxide layer.

Figure 3.10. Equivalent circuit model for the $\mathrm{Ta}_{2} \mathrm{O}_{5}$-ODTS-bilayer and the drop of solution. $R$ : Resistance of the measurement solution, $C$ : Capacitance of the bilayer.

Figure 3.11. Optical image of the electrochemical cell for cyclic voltammetry (CV) measurements in an aqueous droplet connecting three electrodes in oil. WE: LVEWOD electrode as working electrode, CE: platinum wire as counter electrode, RE: sintered $\mathrm{Ag} / \mathrm{AgCl}$ wire as reference electrode.

Figure 3.12. Schematic setup of the integrated LV-EWOD-EIS system associated with optical imaging. Modular setup with (I) measurement cell, (II) electroanalytical instrument, (III) optical imaging instrument and (IV) software.

Figure 3.13. Measurement cell setup and its disassembled components: a LV-EWOD electrode (I.1), an electrode holder (I.2), a platinum electrode (I.3), a glass cuvette (I.4), a transparent hood (I.5) and a holding bar (I.6)....

Figure 3.14. Recorded image of the intersection region of a $3 \mu \mathrm{L}$ droplet on the EWOD electrode. Image analysis with the baseline and the two tangent lines yields the right and the left contact angles as well as the diameter of the contact area. .....53

Figure 3.15. Transimpedance amplifier with a changeable feedback resistor, connections to the working electrode (WE) and the counter electrode (CE) as well as connections to the three channels of the Handyscope. AWG: arbitrary waveform generation, Ch1: Channel 1 for recording the applied signal, Ch2: Channel 2 for recording the response signal

Figure 4.1. Layer model for the object under investigation (Figure 2.11) and overview of the layer-oriented chapter assignment concerning characterization of EWOD-relevant parameters for each dielectric layer individually and for the entire MDS. 60 
Figure 4.2. AFM images with their color-height correlations for (a) anodic $\mathrm{Ta}_{2} \mathrm{O}_{5}$ layer $\left(\boldsymbol{U}_{\text {anod }}=30 \mathrm{~V}\right)$ with a root-mean-square roughness of $0.31 \mathrm{~nm}$, (b) anodic $\mathrm{Ta}_{2} \mathrm{O}_{5}$ layer $\left(\boldsymbol{U}_{\mathrm{anod}}=50 \mathrm{~V}\right)$ with a root-mean-square roughness of $0.47 \mathrm{~nm}$. 62

Figure 4.3. Optical image of the electrode substrates with $\mathrm{T}_{2} \mathrm{O}_{5}$ layer anodized in the round middle area at various voltages $(20-50 \mathrm{~V})$.

Figure 4.4. $\Delta / \Psi$ trajectory for the experimental data points (red) of the anodic $\mathrm{Ta}_{2} \mathrm{O}_{5}$ layers $\left(\boldsymbol{U}_{\text {anod }}=20-50 \mathrm{~V}\right)$ and the scaled theoretical thickness (black curve with dots) of the ellipsometry model (Chapter 3.2.3, Table 3.6).

Figure 4.5. Thickness of $\mathrm{Ta}_{2} \mathrm{O}_{5}$ layers anodized at different voltages $(20-50 \mathrm{~V}, \mathbf{N} \geq 3)$ and a linear fit (red).

Figure 4.6. (a) Bode plot of impedance spectra with impedance modulus (|Z|, dots) and phase shift $\left(\boldsymbol{\sigma}\right.$, circles) against frequency for a native tantalum oxide layer $\left(\boldsymbol{U}_{\text {anod }}=0 \mathrm{~V}\right)$ and for anodic $\mathrm{Ta}_{2} \mathrm{O}_{5}$ layers $\left(\boldsymbol{U}_{\text {anod }}=20-50 \mathrm{~V}\right)$. Grey arrows: critical frequencies for a native oxide layer and for one anodized at $20 \mathrm{~V}$. (b) Equivalent circuit model for data fitting. $R_{1}$ : resistance of the surrounding solution, $C_{2}$ : capacitance of the object under study, $R_{2}$ : resistance of the $\mathrm{Ta}_{2} \mathrm{O}_{5}$ layer. .

Figure 4.7. Capacitance per unit area of the anodic $\mathrm{Ta}_{2} \mathrm{O}_{5}$ layers $\left(\boldsymbol{U}_{\text {anod }}=20-50 \mathrm{~V}, \boldsymbol{N} \geq 3\right)$ fitted to the function, $y=a / x+b$, in red.

Figure 4.8. Relative permittivity of $\mathrm{Ta}_{2} \mathrm{O}_{5}$ layers anodized at different voltages $(20-50 \mathrm{~V}$, $N \geq 3$ )

Figure 4.9. AFM image besides the color-height correlation for an ODTS coating with a rootmean-square roughness of $1.45 \mathrm{~nm}$ on a $\mathrm{Ta}_{2} \mathrm{O}_{5}$ initial layer $\left(\boldsymbol{U}_{\text {anod }}=30 \mathrm{~V}\right) \ldots \ldots . .71$

Figure 4.10. Capacitance per unit area for the ODTS- $\mathrm{Ta}_{2} \mathrm{O}_{5}$-bilayer determined with EIS using a single sinusoidal signal $\left(\boldsymbol{U}_{\mathbf{p p} / \mathbf{2}}=0.5 \mathrm{~V}\right)$ on a $3 \mu \mathrm{L}$ drop of $200 \mathrm{mM} \mathrm{KCl}$ resting on the LV-EWOD electrode $(\boldsymbol{N}=3)$.

Figure 4.11. Cyclic voltammogram of the MDS during the charging and the discharging process (progression shown by orange arrows). Measurement at a scan rate of $32 \mathrm{mV} / \mathrm{s}$ with a $10 \mu \mathrm{L}$ drop of $200 \mathrm{mM} \mathrm{KCl}$ resting in oil on the LV-EWOD electrode (averaged data of 3 cycles).

Figure 4.12. Capacitance per unit area of the $\operatorname{MDS}(\boldsymbol{N}=5)$ and that of the ODTS- $\mathrm{Ta}_{2} \mathrm{O}_{5}$-bilayer $(\boldsymbol{N}=3$, Figure 4.10) determined with EIS using a single sinusoidal signal $\left(\boldsymbol{U}_{\mathbf{p p} / \mathbf{2}}=0.5 \mathrm{~V}\right)$ on a $3 \mu \mathrm{L}$ drop of $200 \mathrm{mM} \mathrm{KCl}$ resting on the LV-EWOD electrode.

Figure 4.13. Section overview with the optical image of the system under study, its equivalent circuit model (reprint of Figure $2.12 \mathrm{~b}$, Chapter 2.2.2.1) and the adapted YoungLippmann equation (Eq. 2.32). Three EWOD-relevant variables (blue): effective voltage drop on the MDS ( $\left.\boldsymbol{U}_{(\mathbf{R M S}) \text { MDS }}\right)$, MDS-capacitance per unit area $\left(\boldsymbol{C}_{\text {MDS }} / \boldsymbol{A}\right)$ and contact angle under voltage $\left(\boldsymbol{\theta}_{\mathbf{u}}\right) \cdot \beta$ : correlation coefficient for assessment of the EWOD effect. .86 
Figure 4.14. Impedance spectra of impedance modulus against frequency (a) during EWOD at varied voltage amplitudes $\left(\boldsymbol{U}_{\mathbf{p p} / 2}=0.5-5 \mathrm{~V}\right)$ on a droplet of $6.25 \mathrm{mM} \mathrm{KCl}$ and (b) during EWOD of droplets with diverse $\mathrm{KCl}$ concentrations $(\boldsymbol{c}=6.25-200 \mathrm{mM}$ ) at the voltage amplitude of $5 \mathrm{~V}$. Grey arrows: critical frequencies dividing the impedance spectra in the capacitive and the resistive response of the system under investigation.

Figure 4.15. Percentage of the RMS-voltage drop on the MDS versus frequency (a) for EWOD at alternating voltages from $0.5 \mathrm{~V}$ to $5 \mathrm{~V}$ on a droplet of $6.25 \mathrm{mM} \mathrm{KCl}$ and (b) for EWOD on droplets with $\mathrm{KCl}$ concentrations from $200 \mathrm{mM}$ to $6.25 \mathrm{mM}$ at the alternating voltage of $5 \mathrm{~V}$. Black arrow with $c$ : increase of $\mathrm{KCl}$ concentration.....89

Figure 4.16. MDS capacitance per unit contact area to the droplet versus frequency for EWOD at alternating voltages from $0.5 \mathrm{~V}$ to $5 \mathrm{~V}$ on a droplet of $6.25 \mathrm{mM} \mathrm{KCl}$. Black arrow with $\boldsymbol{U}$ : increase of the voltage.

Figure 4.17. (a) Optical image of a $3 \mu \mathrm{L}$ droplet in tetradecane. $\boldsymbol{\theta}_{\mathbf{u}}$ : contact angle under voltage. (b) Contact angle under voltage versus frequency for the electrowetting at alternating voltages from $0.5 \mathrm{~V}$ to $5 \mathrm{~V}$ on a droplet of $6.25 \mathrm{mM} \mathrm{KCl}$. Black arrow with $\boldsymbol{f}_{\mathrm{t}}$ : frequency threshold.

Figure 4.18. (a) Plot of the adapted Young-Lippmann equation (Equation 2.32) for the EWOD at frequencies from $100 \mathrm{~Hz}$ to $1 \mathrm{MHz}$ on a droplet of $100 \mathrm{mM} \mathrm{KCl}$. (b) Correlation coefficient $\beta$ for droplets of $100 \mathrm{mM} \mathrm{KCl}$ and $6.25 \mathrm{mM} \mathrm{KCl}$ at frequencies where $\boldsymbol{\beta}$ $<1(N=3)$.

Figure 4.19. (a) EWOD system model (Chapter 2.2.1.3): an aqueous saline droplet on the MDS (multilayer dielectric stack) consisting of an interfacial oil layer and solid dielectrics (b) Overview of the time-resolved response of the contact area (red), the contact angle (blue) of the droplet and the area-related MDS-capacitance (green) to the staircase voltage stimulus (black). The arbitrary unit is defined for each parameter as the ratio to the difference between its maximum and its minimum value........96

Figure 4.20. Non-linear voltage dependence of the electric potential energy per unit area $\left(\mathrm{E}_{\mathrm{el}} / \mathrm{A}\right)$ in the ascending (red) and in the descending (blue) voltage ramp. $\boldsymbol{U}_{\text {off: }}$ voltage offsets of the staircase stimulus 98

Figure 4.21. Non-linear responses of the MDS-capacitance per unit area $\left(\boldsymbol{C}_{\mathrm{MDS}} / \boldsymbol{A}\right)$ to the ascending (red) and to the descending (blue) voltage ramp. $\boldsymbol{U}_{\text {off: }}$ : voltage offsets of the staircase stimulus.

Figure 4.22. Non-linear variation of the oil layer thickness $\left(\boldsymbol{d}_{\mathbf{o i l}}\right)$ with the electric potential energy per unit area $\left(\boldsymbol{E}_{\mathrm{el}} / \boldsymbol{A}\right)$ in the ascending (red) and in the descending (blue) voltage ramp.

Figure 4.23. Voltage threshold $\left(\boldsymbol{U}_{\boldsymbol{t}, \boldsymbol{\theta}}\right)$ of EWOD effect determined with the contact angle $\left(\boldsymbol{\theta}_{\mathbf{u}}\right)$ response to the ascending voltage ramp. $\boldsymbol{U}_{\text {off: }}$ voltage offsets of the staircase stimulus.

Figure 4.24. Voltage threshold $\left(\boldsymbol{U}_{t, \boldsymbol{A}}\right)$ of EWOD effect determined with the contact area $(\boldsymbol{A})$ response to the ascending voltage ramp. $\boldsymbol{U}_{\text {off: }}$ voltage offsets of the staircase stimulus. 102 
Figure 4.25. Cosine of the contact angle $\left(\cos \theta_{\mathrm{u}}\right)$ at diverse levels of the areal electrical potential energy $\left(\boldsymbol{E}_{\mathrm{el}} / \boldsymbol{A}\right)$ in the ascending (red) and the descending (blue) voltage ramp. Stage I: electrowetting; Stage II (enclosed square): inertia behavior in the switch from electrowetting to dewetting; Stage III: dewetting. Dashed lines: linear fitting lines $\left(\overline{\boldsymbol{R}}^{2}=0.999\right.$ within Stage I, $\overline{\boldsymbol{R}}^{2}=0.994$ at Stage III).......................... 103

Figure 4.26. Time-resolved dynamic relaxation process of the contact area $(\boldsymbol{A})$ upon the voltage jump from $4.14 \mathrm{~V}$ to $4.42 \mathrm{~V}$. 105

Figure 4.27. Non-linear correlation between the characteristic time $(\boldsymbol{\tau})$ and the electrical potential energy per unit area $\left(\boldsymbol{E}_{\mathrm{el}} / \boldsymbol{A}\right)$ for each voltage step in the ascending (red) and the descending (blue) voltage ramp. 106

Figure A.1. Increase of the droplet contact area to its equilibrium end state upon a voltage step applied for a sufficient long period. 123 


\subsection{List of Tables}

Table 3.1. Parameters for the PEIS measurements of the $\mathrm{Ta}_{2} \mathrm{O}_{5}$ layer with EC-lab. 36

Table 3.2. Parameters for the data fit with EC-lab.

Table 3.3. Parameters for the PEIS measurements of the ODTS-Ta2O5-bilayer with EC-lab.

Table 3.4. Parameters for the CV measurements with EC-lab.

Table 3.5. Parameters for the ellipsometric measurements of $\mathrm{Ta}_{2} \mathrm{O}_{5}$ layers and ODTS coating.

Table 3.6. Refractive index $(\boldsymbol{n})$ and extinction coefficient $(\boldsymbol{k})$ of each layer for the analysis model.

Table 3.7. Parameter settings for the AFM imaging of a $\mathrm{Ta}_{2} \mathrm{O}_{5}$ surface (left) and for that of an ODTS surface (right).

Table 3.8. Parameters for the contact angle measurements.

Table 3.9. Parameters for the $\mathrm{CAH}$ measurements with the dynamic sessile drop method. .47

Table 3.10. Functionality and specification of the hardware components in the measurement cell.

Table 3.11. Hardware components of two instrumental setups corresponding to measurement applications with frequency-domain EIS and with dynamic EIS respectively. .....52

Table 3.12. Specification of the Pike high-speed camera. ${ }^{136}$ .53

Table 3.13. $\mathrm{KCl}$ concentrations in the droplet and the correspondingly used feedback resistors.

Table 3.14. Parameter setting for the simultaneous EWOD excitation, EIS in the frequency domain and imaging measurement.

Table 3.15. Parameter setting for the simultaneous EWOD excitation and dynamic EIS. .....59

Table 4.1. Overview of analytic methods or calculation applied to determine the EWODrelevant parameters for each dielectric layer in the MDS. AFM: atomic force microscopy, $\mathrm{CAH}$ : measurement of contact angle hysteresis, CA: contact angle measurement, CV: cyclic voltammetry, EIS: electrical impedance spectroscopy.

Table 4.2. Contact angle and contact angle hysteresis determined for the anodic Ta2O5 surface $\left(\boldsymbol{U}_{\text {anod }}=20-50 \mathrm{~V}\right)$ and for the ODTS surface based on a $\mathrm{Ta}_{2} \mathrm{O}_{5}$ initial layer.

Table 4.3. Contact angle and contact angle hysteresis of a $3 \mu \mathrm{L}$ water droplet on the LV-EWOD electrode in air and in tetradecane.

Table 4.4. Overview table of the EWOD-relevant parameters determined for each dielectric layer in the MDS. $\boldsymbol{R}_{\text {RMS }}$ : root-mean-square roughness, $\mathrm{CAH}$ : contact angle hysteresis, $\boldsymbol{\theta}_{\mathbf{0}}$ : contact angle without voltage application. .82 


\subsection{List of Abbreviations}

AC

AFM

$\mathrm{AOI}$

Arb. unit

AWG

CA

$\mathrm{CAH}$

CE

CV

CV

DC

df

DTS

EDL

EIS

EWOD

IBA

LED

LV-EWOD

LV-EWOD-EIS

ODTS

OTFT

PEIS

PTFE

RE

RMS

ROI

RSS

SAM alternating current

atomic force microscopy

angle of incidence

arbitrary unit

arbitrary waveform generation

contact angle measurement

contact angle hysteresis

counter electrode

cyclic voltammetry

coefficient of variation

direct current

degree of freedom

(n-decly)trichlorosilane

electrochemical double layer

electrical impedance spectroscopy

electrowetting on dielectrics

Institute for Bioprocessing and Analytical Measurement Techniques

Light-emitting diode

low-voltage electrowetting on dielectrics

low-voltage electrowetting on dielectrics integrated with electrical impedance spectroscopy

octadecyltrichlorosilane

organic thin-film transistors

potentio electrochemical impedance spectroscopy

Polytetrafluoroethylene

reference electrode

root mean square

region of interest

residual sum of squares

self-assembled monolayer 
STED

TSS

WE stimulated emission depletion

total sum of squares

working electrode 


\subsection{List of Symbols}

\begin{tabular}{|c|c|}
\hline $\boldsymbol{A}$ & contact area \\
\hline$A_{e}$ & contact area at the interfacial equilibrium state \\
\hline $\boldsymbol{C}$ & capacitance, capacitor \\
\hline bilayer & capacitance of the solid dielectric bilayer \\
\hline$C_{\mathrm{dl}}$ & capacitance of the electrochemical double layer \\
\hline drop & droplet capacitance \\
\hline$C_{\text {MDS }}$ & total capacitance of the multilayer dielectric stack \\
\hline$C_{\text {oil }}$ & oil film capacitance \\
\hline silane & silane layer capacitance \\
\hline $\mathrm{Ta}_{2} \mathrm{O}_{5}$ & $\mathrm{Ta}_{2} \mathrm{O}_{5}$-layer capacitance \\
\hline $\boldsymbol{c}$ & $\mathrm{KCl}$ concentration in the droplet \\
\hline$d$ & layer thickness \\
\hline$d_{\mathrm{c}}$ & diameter of the contact area \\
\hline$d_{\text {oil }}$ & oil layer thickness \\
\hline $\boldsymbol{E}_{\text {el }}$ & electric potential energy \\
\hline$f$ & frequency \\
\hline$f_{\mathrm{c}}$ & critical frequency \\
\hline$f_{t}$ & frequency threshold \\
\hline $\boldsymbol{G}$ & Gibbs energy \\
\hline $\boldsymbol{I}$ & current \\
\hline $\mathbf{p p} / 2$ & current amplitude \\
\hline$j$ & imaginary unit \\
\hline $\boldsymbol{k}$ & extinction coefficient \\
\hline $\boldsymbol{l}$ & length \\
\hline$m$ & number of $\mathrm{CH}_{2}$-units in the alkyl chain \\
\hline$n_{a}$ & amount of substance \\
\hline
\end{tabular}




\begin{tabular}{|c|c|}
\hline $\boldsymbol{n}$ & reflection index \\
\hline$N$ & number of measurements \\
\hline$p$ & pressure \\
\hline$Q$ & charge \\
\hline $\boldsymbol{R}$ & resistance, resistor \\
\hline$\overline{\boldsymbol{R}}^{2}$ & adjusted coefficient of determination \\
\hline$R^{P}$ & $\begin{array}{l}\text { reflection coefficient of the } p \text { component (parallel to the } \\
\text { incident plane) in ellipsometry }\end{array}$ \\
\hline$R^{S}$ & $\begin{array}{l}\text { reflection coefficient of the s component (perpendicular to the } \\
\text { incident plane) in ellipsometry }\end{array}$ \\
\hline$R_{\text {drop }}$ & droplet resistance \\
\hline$R_{\mathrm{MDS}}$ & total resistance of the multilayer dielectric stack \\
\hline$R_{R M S}$ & root-mean-square value of surface roughness \\
\hline$t$ & time \\
\hline$t_{\mathrm{m}}$ & monolayer thickness \\
\hline$T$ & period \\
\hline $\boldsymbol{U}$ & applied voltage \\
\hline$U_{\text {anod }}$ & anodization voltage \\
\hline$U_{C}$ & voltage drop across a capacitor \\
\hline$U_{\text {off set }}$ & voltage offset \\
\hline $\boldsymbol{U}_{\mathrm{off}, \min }$ & minimum voltage offset \\
\hline$U_{\text {off,max }}$ & maximum voltage offset \\
\hline$U_{\mathrm{pp} / 2}$ & voltage amplitude \\
\hline$U_{R}$ & voltage drop across a resistor \\
\hline$U_{\mathrm{RMS}}$ & root-mean-square value of the applied voltage \\
\hline$U_{(\mathrm{RMS}) \mathrm{MDS}}$ & $\begin{array}{l}\text { effective voltage drop on the MDS in the root-mean-square } \\
\text { value }\end{array}$ \\
\hline$U_{\mathrm{t}}$ & EWOD voltage threshold \\
\hline$U_{t, A}$ & EWOD voltage threshold determined with the contact area \\
\hline
\end{tabular}




\begin{tabular}{|c|c|}
\hline $\boldsymbol{U}_{t, \boldsymbol{\theta}}$ & EWOD voltage threshold determined with the contact angle \\
\hline$V_{\text {in }}$ & voltage signal input \\
\hline$V_{\text {out }}$ & voltage signal output \\
\hline$X_{\mathbf{c}}$ & capacitive reactance \\
\hline$X_{C_{\text {drop }}}$ & capacitive reactance of the droplet \\
\hline$X_{C_{\text {MDS }}}$ & capacitive reactance of the multilayer dielectric stack \\
\hline $\boldsymbol{Z}$ & complex impedance \\
\hline$|\boldsymbol{Z}|$ & impedance modulus \\
\hline$Z_{I m}$ & imaginary part of the complex impedance \\
\hline$Z_{R e}$ & real part of the complex impedance \\
\hline$\alpha$ & cross-section area for resistance calculation \\
\hline $\boldsymbol{\beta}$ & $\begin{array}{l}\text { correlation-coefficient introduced into the adapted Young- } \\
\text { Lippmann equation for assessment of the electrowetting } \\
\text { efficiency }\end{array}$ \\
\hline$\gamma_{\text {If }}$ & liquid-fluid interfacial tension \\
\hline$\gamma_{\mathrm{sf}}$ & solid-fluid interfacial tension \\
\hline$\gamma_{\mathbf{s l}}$ & solid-liquid interfacial tension \\
\hline$\gamma_{\mathrm{sl}(\mathbf{0})}$ & solid-liquid interfacial tension under zero voltage \\
\hline$\gamma_{\mathbf{s l}(U)}$ & solid-liquid interfacial tension under voltage \\
\hline$\Delta$ & $\begin{array}{l}\text { phase shift difference between the } p \text { and the } s \text { component in } \\
\text { ellipsometry }\end{array}$ \\
\hline$\varepsilon_{0}$ & vacuum permittivity \\
\hline$\varepsilon_{d}$ & relative permittivity \\
\hline $\boldsymbol{\theta}$ & static contact angle \\
\hline $\boldsymbol{\theta}_{0}$ & contact angle under zero voltage \\
\hline $\boldsymbol{\theta}_{\mathrm{adv}}$ & advancing contact angle \\
\hline $\boldsymbol{\theta}_{\text {rec }}$ & receding contact angle \\
\hline
\end{tabular}




\begin{tabular}{|c|c|}
\hline $\boldsymbol{\theta}_{\mathbf{u}}$ & contact angle under voltage \\
\hline$\Delta \boldsymbol{\theta}$ & contact angle hysteresis \\
\hline$\Theta$ & Temperature \\
\hline$\lambda$ & wavelength \\
\hline $\boldsymbol{\mu}$ & chemical potential \\
\hline $\bar{\mu}$ & mean \\
\hline$\rho$ & resistivity \\
\hline$\rho_{\text {sl }}$ & charge density on the solid-liquid interface \\
\hline$\sigma$ & standard deviation \\
\hline$\tau$ & characteristic time \\
\hline$\phi$ & $\begin{array}{l}\text { phase shift between the voltage stimulus and current } \\
\text { response in EIS }\end{array}$ \\
\hline $\boldsymbol{\psi}$ & amplitude reduction in ellipsometry \\
\hline$\omega$ & angular frequency \\
\hline$\omega_{c}$ & critical frequency \\
\hline
\end{tabular}




\subsection{Circuit Diagram of Transimpedance Amplifier}
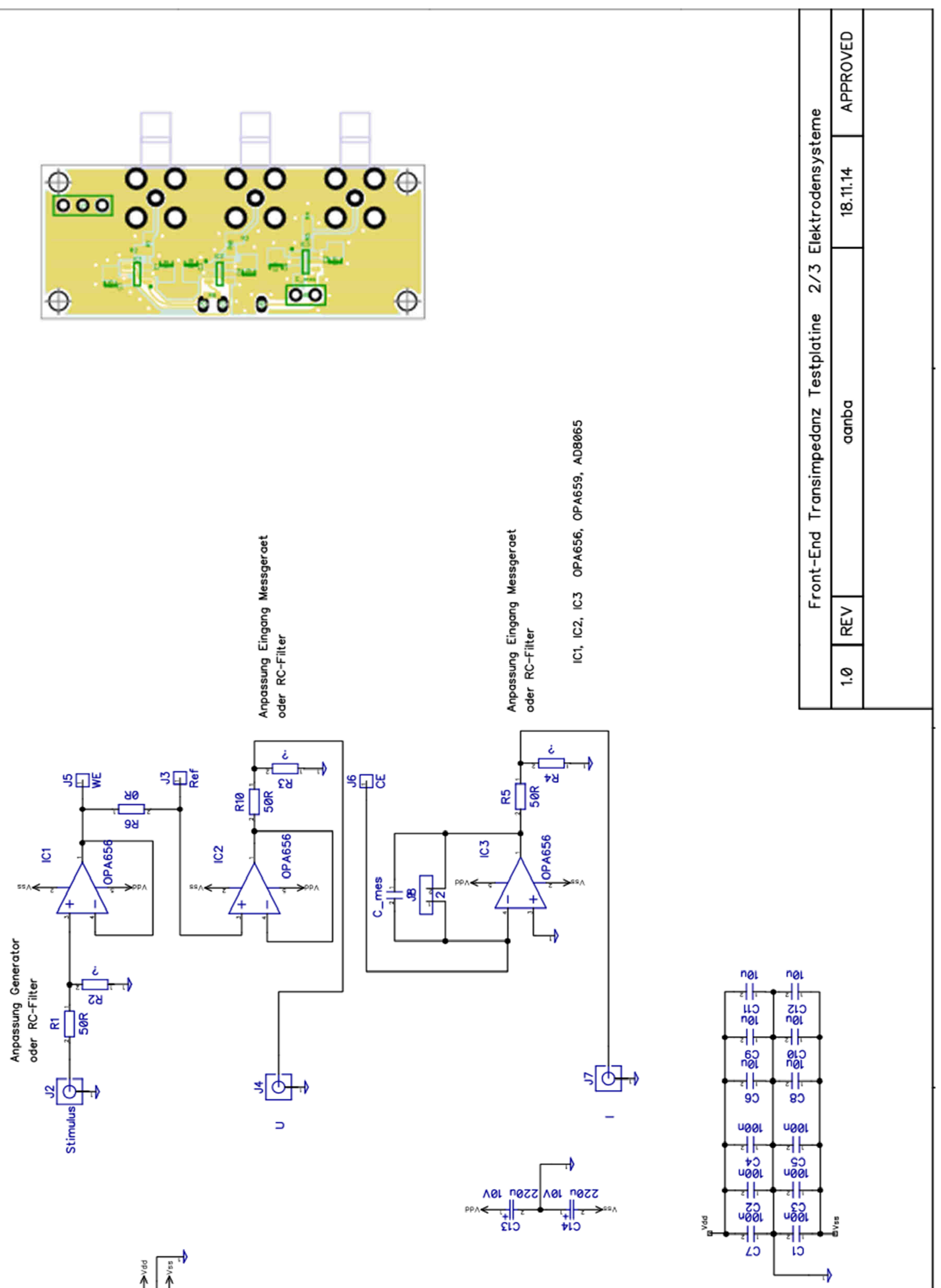


\subsection{Technical Drawings}

\begin{tabular}{|c|c|c|}
\hline Drawing Nr. & Component name & Relevance \\
\hline Li00 & Specimen holder & Figure 3.3, Chapter 3.1.1 \\
\hline Li01 & Anodization cell & Figure 3.5, Chapter 3.1.2 \\
\hline Li02 & Camera holder & Chapter 3.3.1 \\
\hline Li03 & Transparent hood & I.5, Figure 3.13, Chapter 3.3.1 \\
\hline Li04 & EWOD-electrode holder & I.2, Figure 3.13, Chapter 3.3.1 \\
\hline Li05 & Sample stage cover & Chapter 3.3.1 \\
\hline Li06 & Sample stage supporter & Chapter 3.3.1 \\
\hline Li07 & $\begin{array}{l}\text { Platinum electrode holder \& } \\
\text { Holding bar }\end{array}$ & $\begin{array}{l}\text { I.3, Figure } 3.13, \text { Chapter } 3.3 .1 \& \\
\text { I.6, Figure } 3.13 \text {, Chapter } 3.3 .1\end{array}$ \\
\hline
\end{tabular}

Li08

Sample stage base

Chapter 3.3.1 

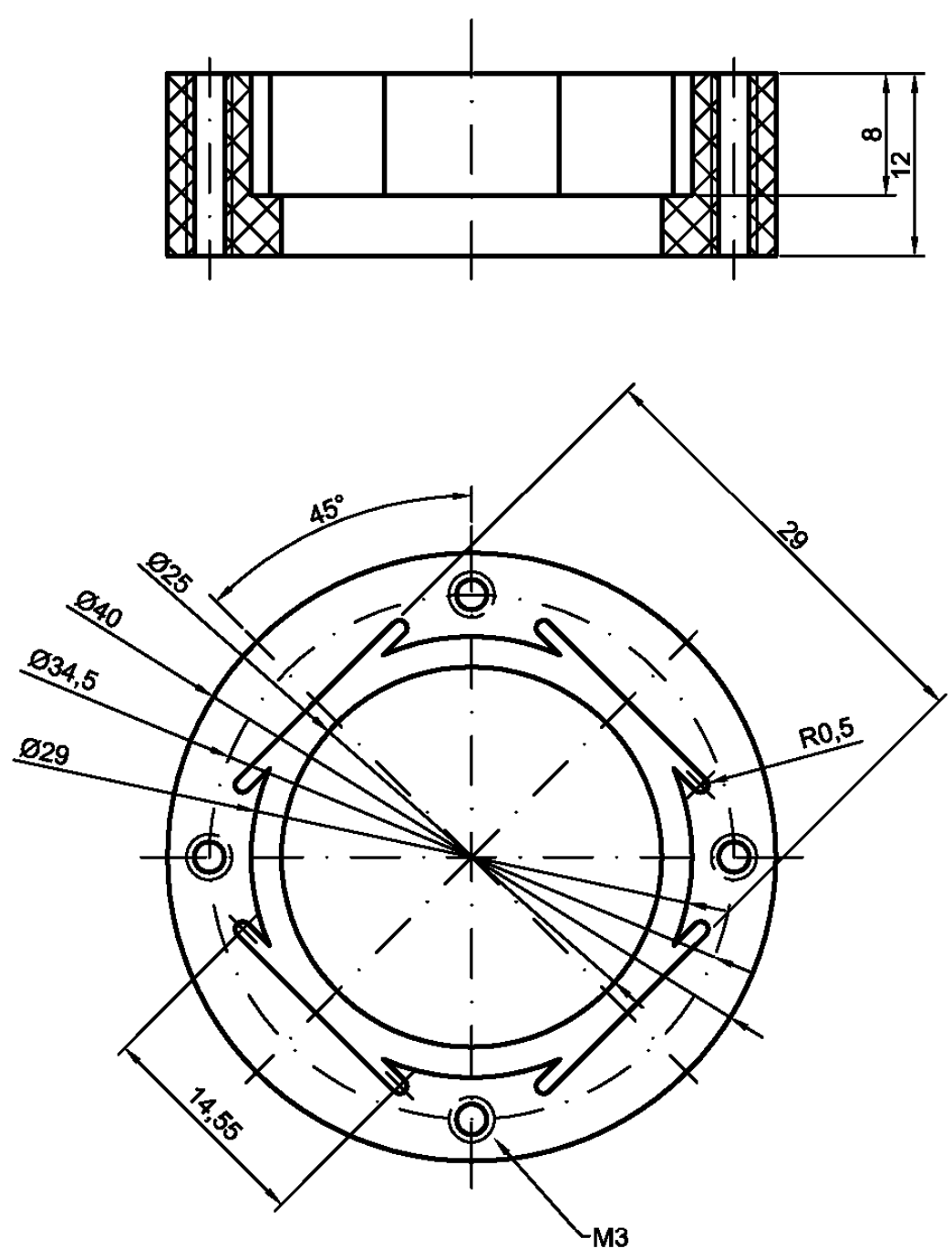

M 2:1

Rd 40 PTFE

01 Stk.

\begin{tabular}{|c|c|c|c|c|c|}
\hline $\begin{array}{l}\text { Verantwort. Abtg. } \\
\text { NFG }\end{array}$ & $\begin{array}{l}\text { Technieche Referenz } \\
860000\end{array}$ & $\begin{array}{l}\text { Errstellt durch } \\
\text { Y. Li; A. Grodrian }\end{array}$ & Genehmlgt von & $\begin{array}{l}\text { Datel } \\
\quad \text { LiO0.dwg }\end{array}$ & \\
\hline & & Dokumentanart & ETZ & Dokumenttanstatus & \\
\hline & & Thtal, Zusäblalicher Titel & & Bauteil-Nr. & \\
\hline & & $\begin{array}{l}\text { Wafel } \\
\text { Mitt }\end{array}$ & $\begin{array}{l}\text { ager- } \\
\text { eil }\end{array}$ & $\begin{array}{l}\text { And. } \\
\text { 2014-07-08 }\end{array}$ & Spr. \\
\hline
\end{tabular}




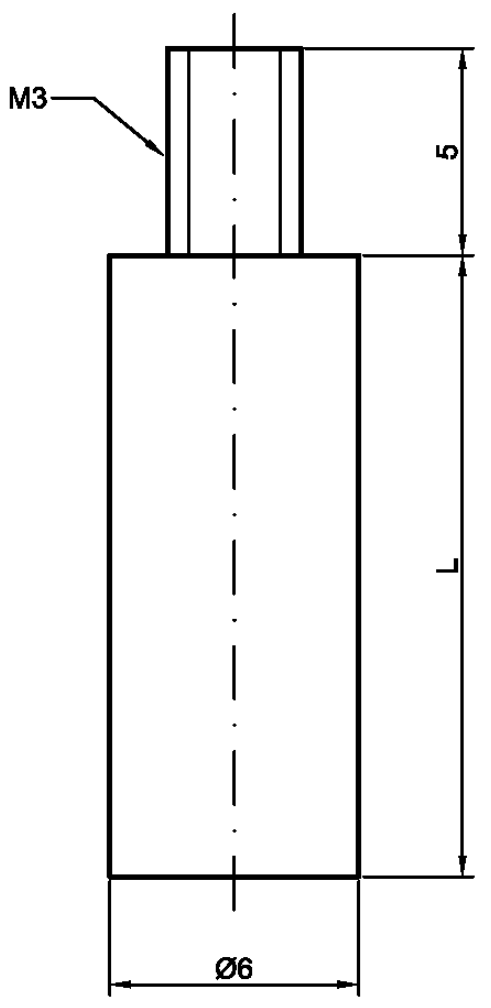

\begin{tabular}{|c||c|c|}
\hline L & 18 & 85 \\
\hline Stk. & 4 & 1 \\
\hline
\end{tabular}

M 5:1

Rd 6 PEEK

04 + 01 Stk.

\begin{tabular}{|c|c|c|c|c|c|}
\hline $\begin{array}{l}\text { Verantwortl. Abtg. } \\
\text { NFG }\end{array}$ & $\begin{array}{l}\text { Technische Referenz } \\
860000\end{array}$ & $\begin{array}{l}\text { Errstallt durch } \\
\text { Y. Li; A. Grodrian }\end{array}$ & Genehmlgt von & ${ }^{\text {Datel }}$ LiO0.dwg & \\
\hline & & | Dokumentanart & ETZ & Dokumentenstatus & \\
\hline & & Tital, zusäbzlicher Titel & & Bauteil-Nr. & \\
\hline & & $\begin{array}{r}\text { Wafer } \\
\text { Stan }\end{array}$ & & $\begin{array}{l}\text { and. Ausgabedatum } \\
\text { 2014-07-08 }\end{array}$ & Spr. \\
\hline
\end{tabular}




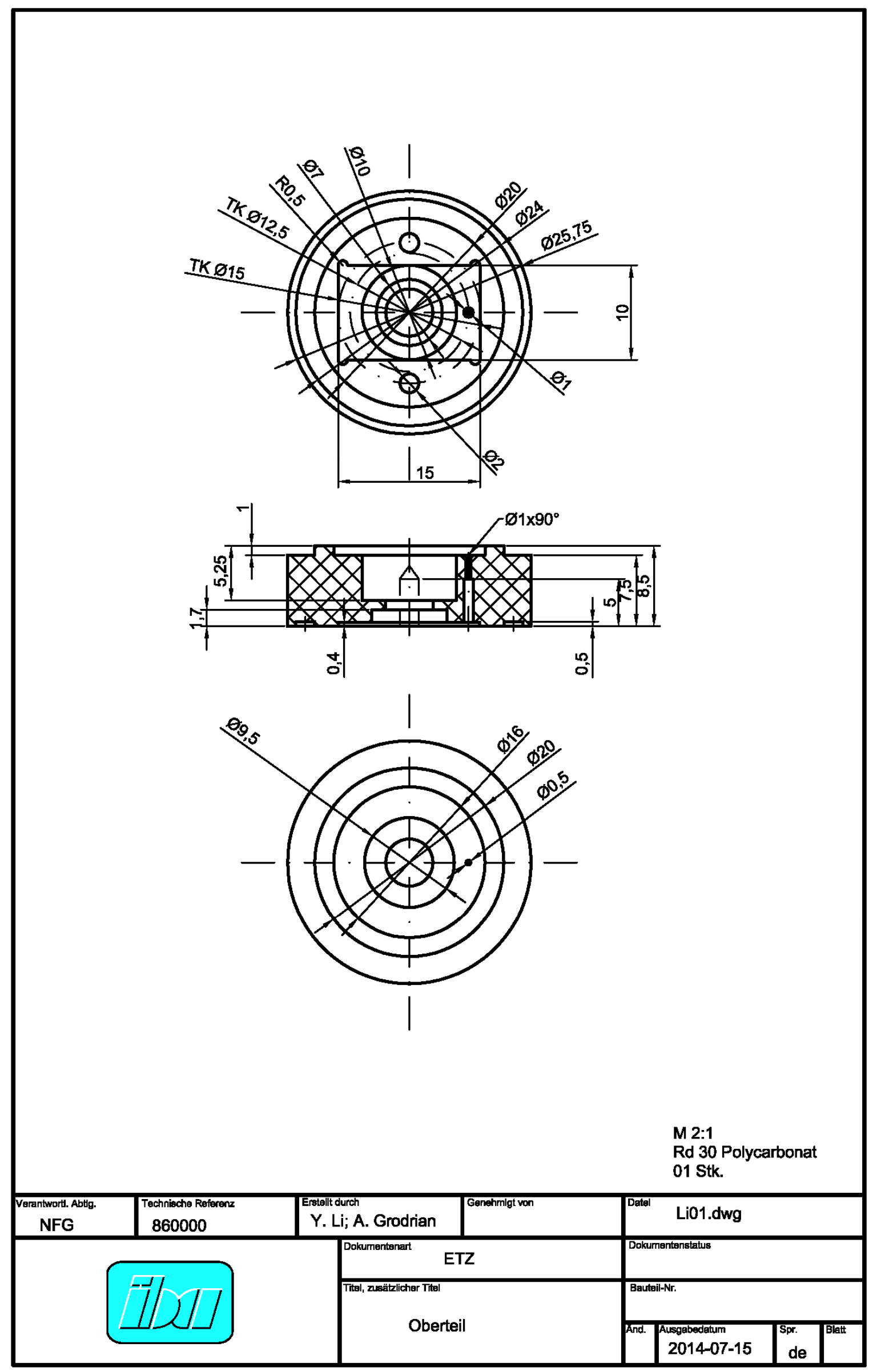




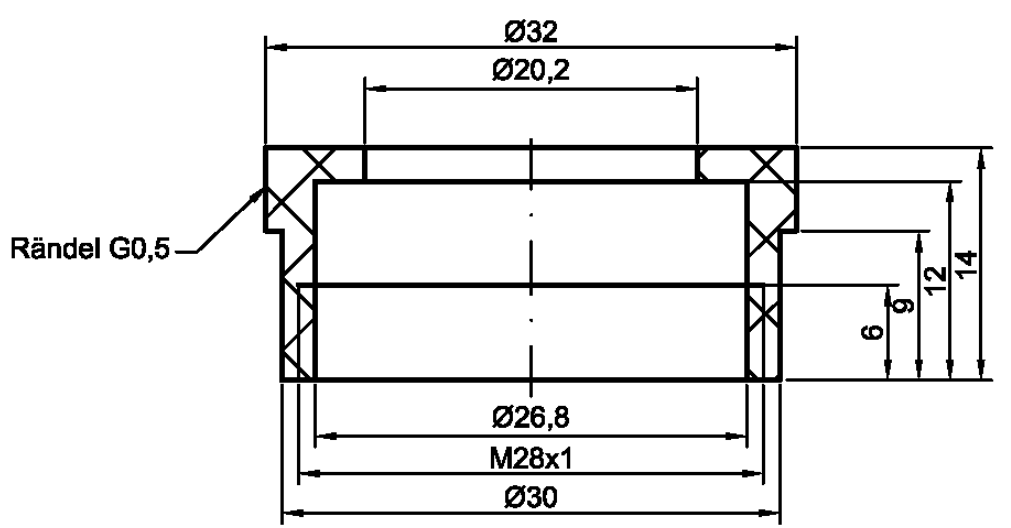

M 2:1

Rd 40 PEEK

01 Stk.

\begin{tabular}{|c|c|c|c|c|c|}
\hline $\begin{array}{l}\text { Verantwortl. Abtg. } \\
\text { NFG }\end{array}$ & $\begin{array}{l}\text { Tecthnische Referenz } \\
860000\end{array}$ & $\begin{array}{l}\text { Erstellt durch } \\
\text { Y. Li; A. Grodrian }\end{array}$ & Genehmlgt von & $\begin{array}{ll}\text { Datel } & \text { Li01.dwg }\end{array}$ & \\
\hline & & \multicolumn{2}{|c|}{$\begin{array}{ll}\text { Dokumentenart } \quad E T Z\end{array}$} & \multicolumn{2}{|l|}{ Dokumentanstzatus } \\
\hline & & \multicolumn{2}{|l|}{ Thtal, zusäblalicher Thtel } & \multicolumn{2}{|l|}{ Bauteili-Nr. } \\
\hline & & \multicolumn{2}{|c|}{ Überwurfmutter } & $\begin{array}{l}\text { And. Ausgabedatum } \\
\text { 2014-07-15 }\end{array}$ & de \\
\hline
\end{tabular}




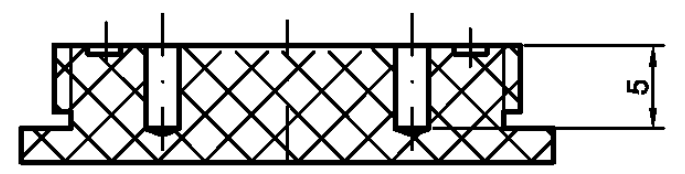

Ansicht $90^{\circ}$ gedreht
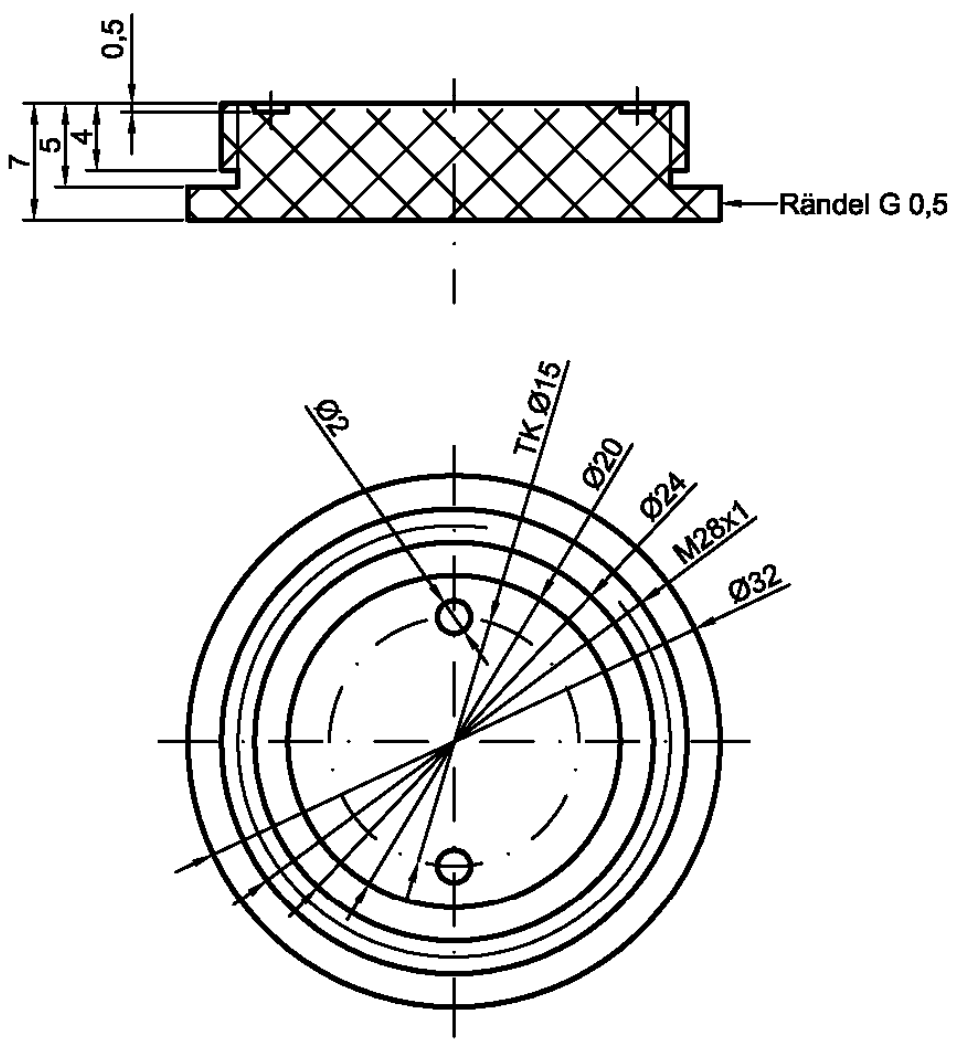

M 2:1

Rd 40 PEEK

01 Stk.

\begin{tabular}{|c|c|c|c|c|c|}
\hline $\begin{array}{c}\text { Verantwortl. Abtg. } \\
\text { NFG }\end{array}$ & $\begin{array}{l}\text { Technische Referenz } \\
860000\end{array}$ & $\begin{array}{l}\text { Eratellt durch } \\
\text { Y. Li; A. Grodrian }\end{array}$ & Genehmlgt von & $\begin{array}{ll}\text { Datel } & \text { Li01.dwg }\end{array}$ & \\
\hline & & \multicolumn{2}{|c|}{$\begin{array}{ll}\text { Dokumentenart } \quad \text { ETZ }\end{array}$} & \multicolumn{2}{|l|}{ Dokumentanstatus } \\
\hline & & \multicolumn{2}{|l|}{ Trital, zusäzalicher Thtel } & \multicolumn{2}{|l|}{ Bauteail-Nr. } \\
\hline & & \multicolumn{2}{|c|}{ Unterteil } & 2014-07-15 & de \\
\hline
\end{tabular}




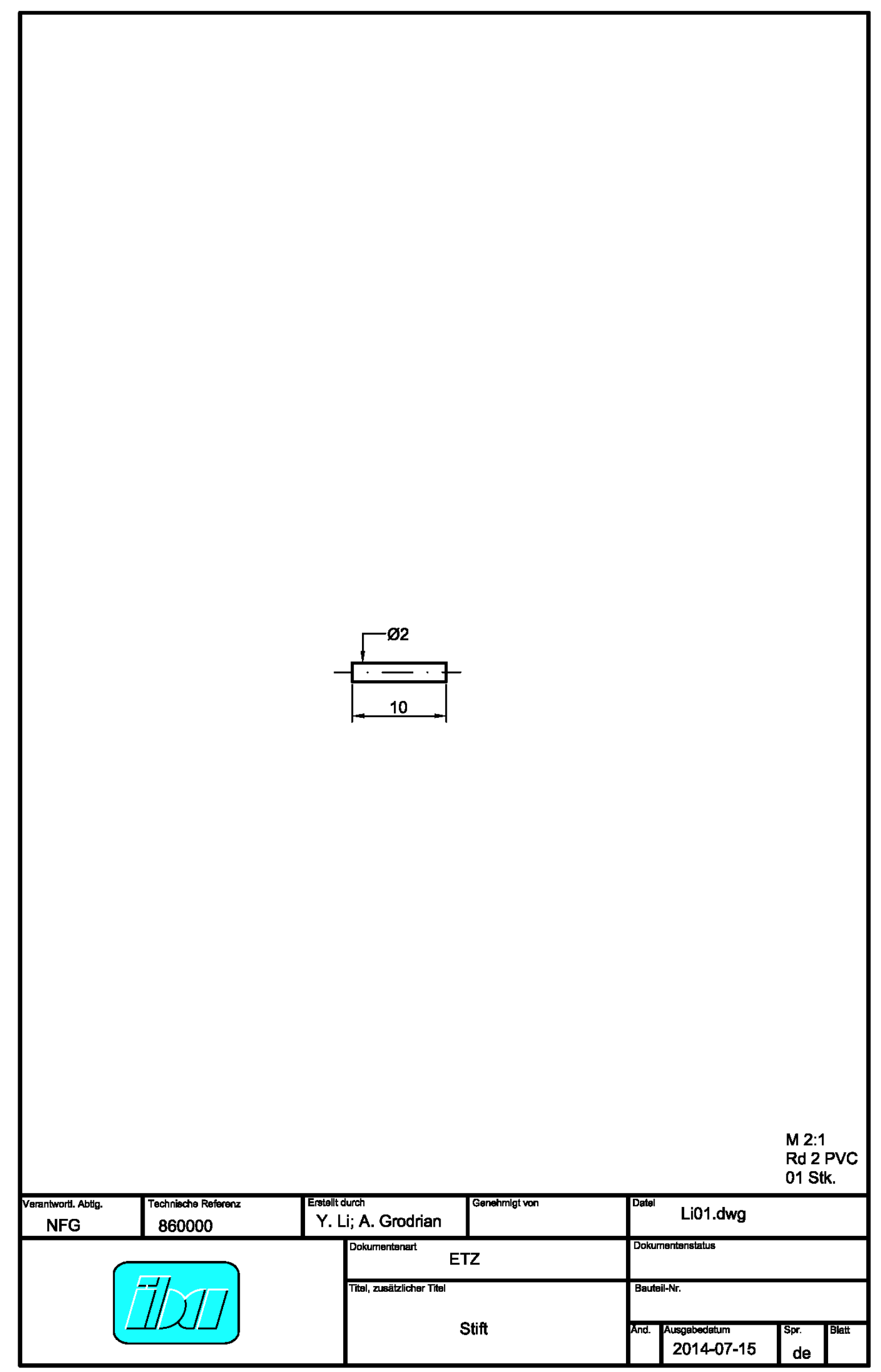




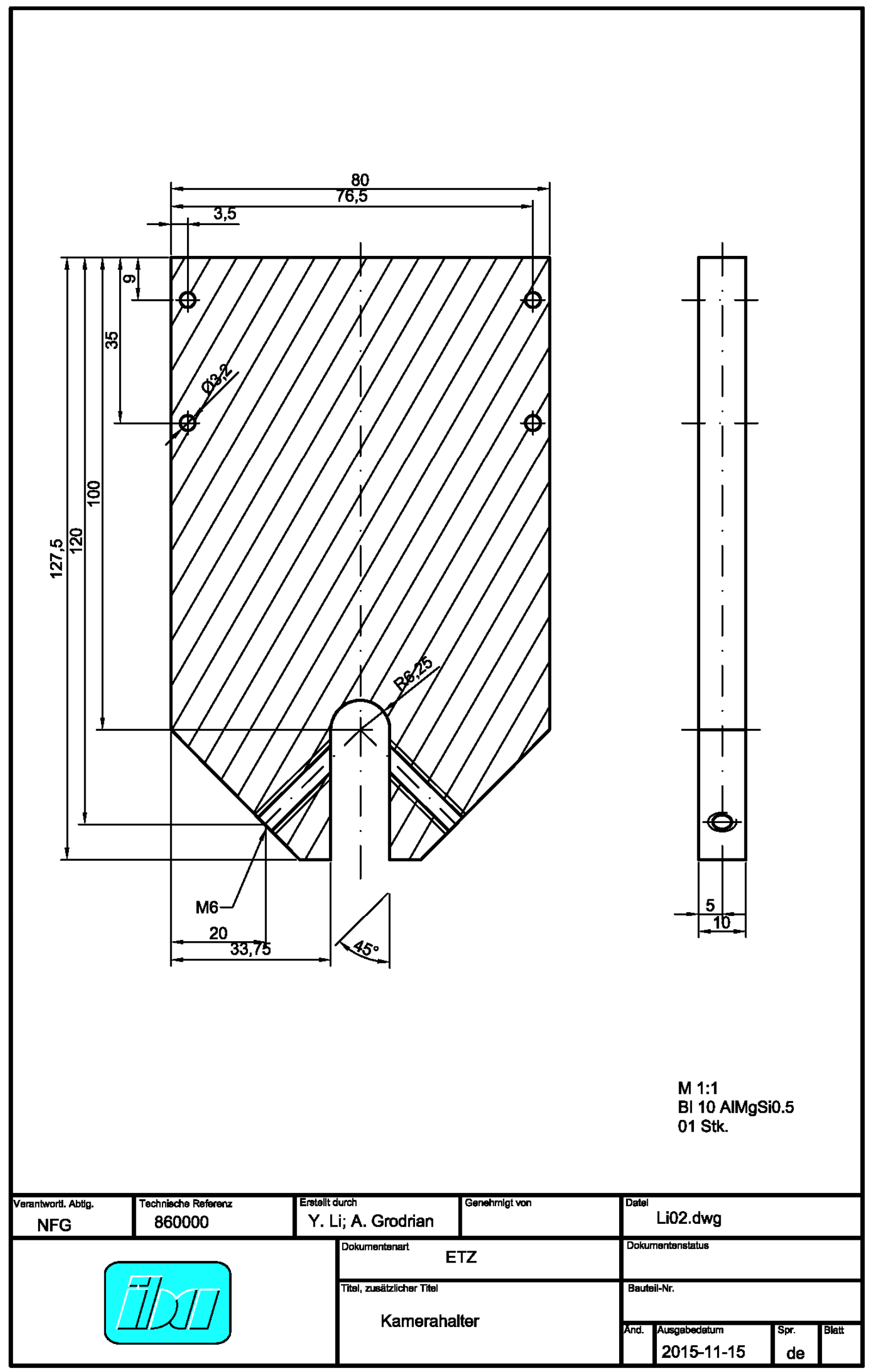




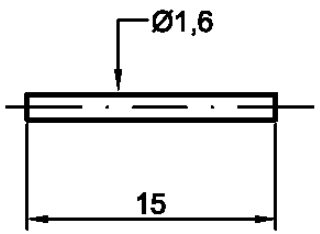

M 2:1

Rd 1,6 Wst.: 1.4301

02 Stk.

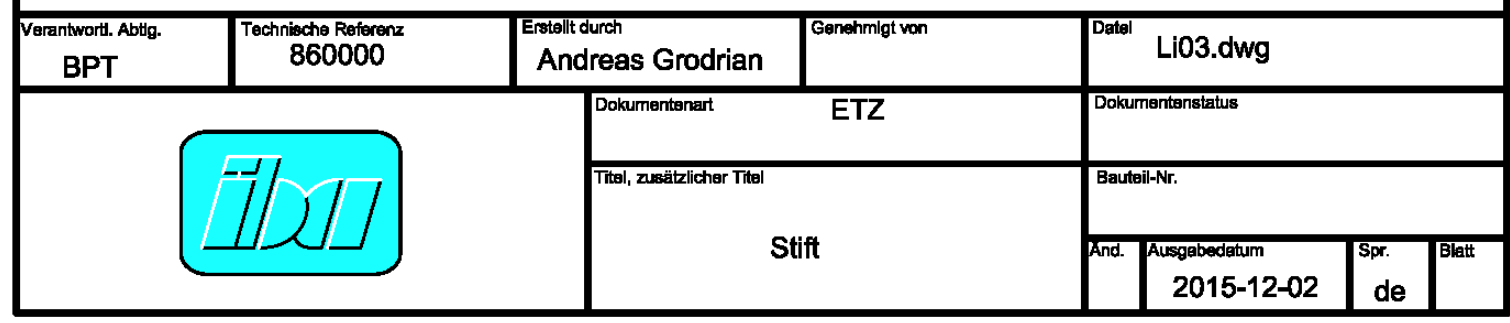



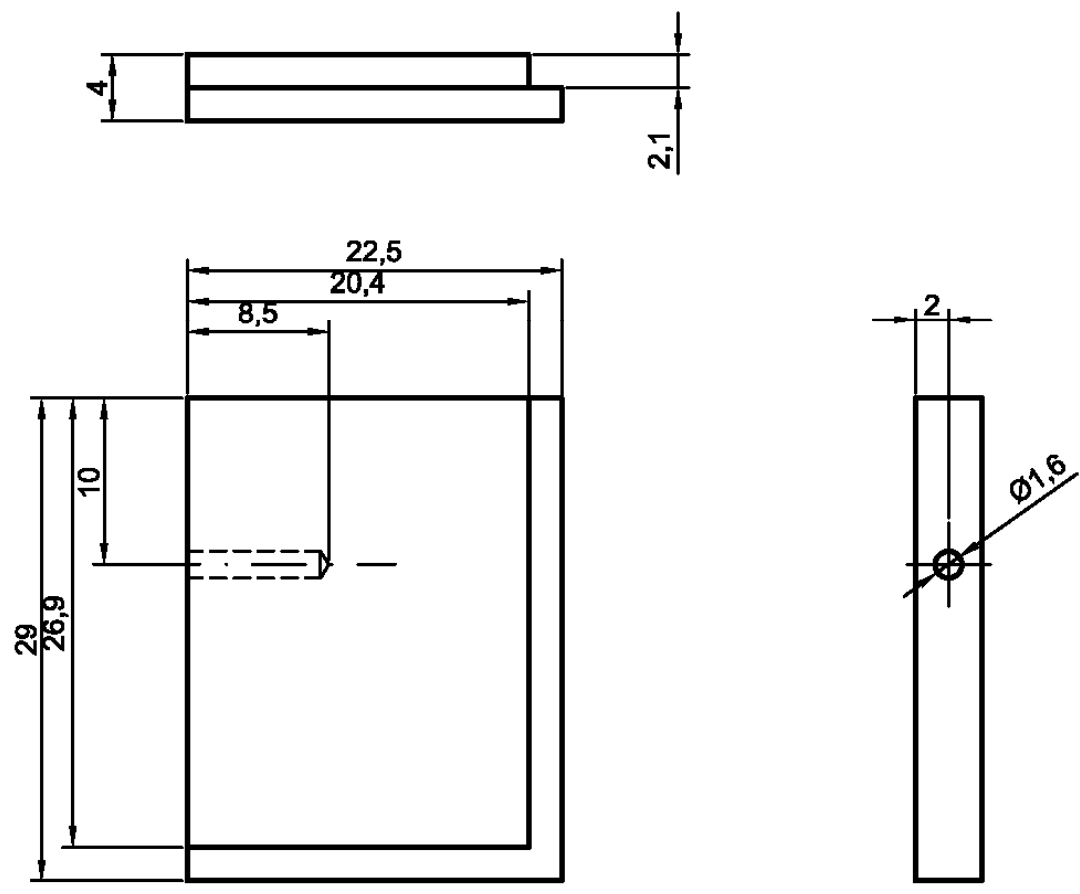

M 2:1

PC-Platte $4 \mathrm{~mm}$ klar 02 Stk.

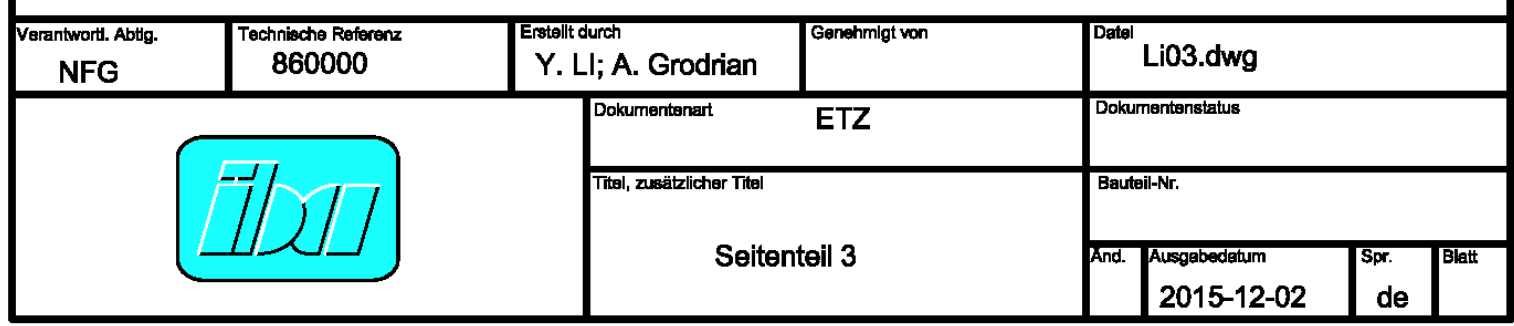



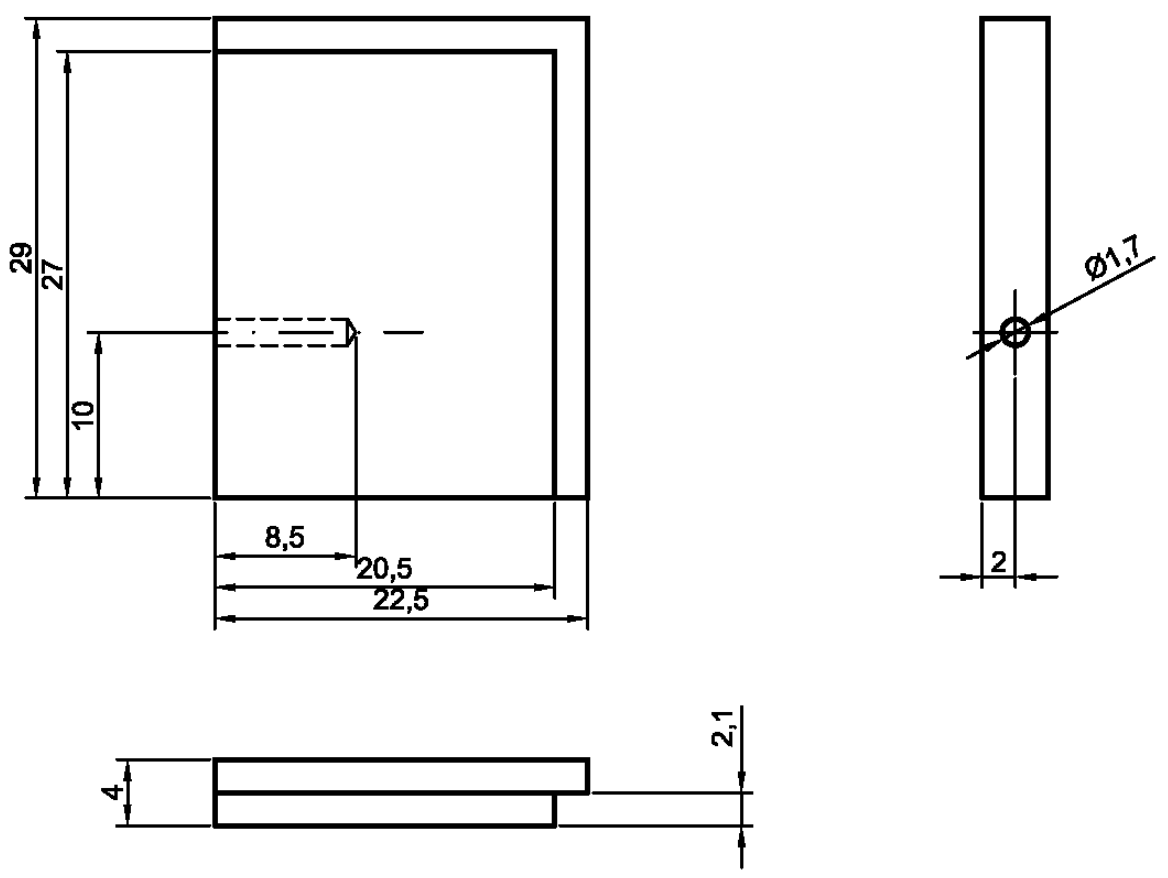

M 2:1

PC-Platte $4 \mathrm{~mm}$ klar 02 Stk.

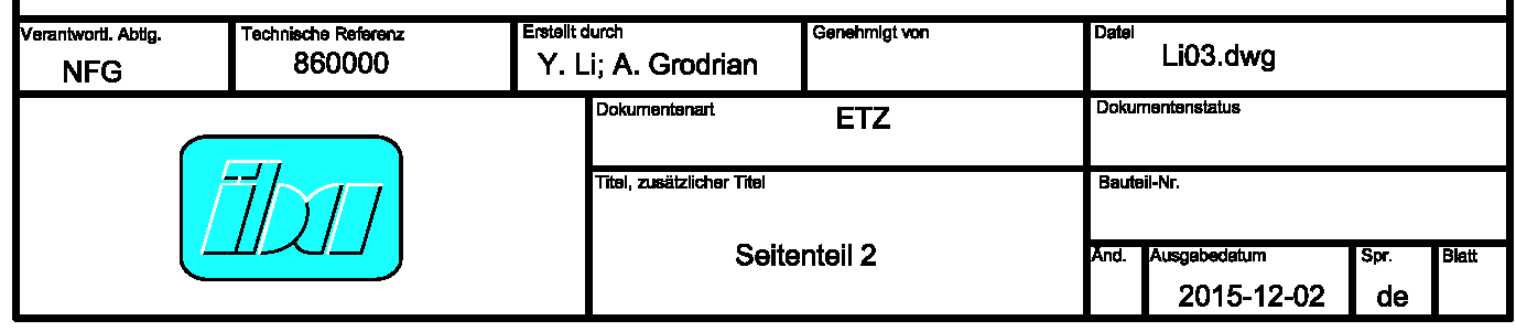



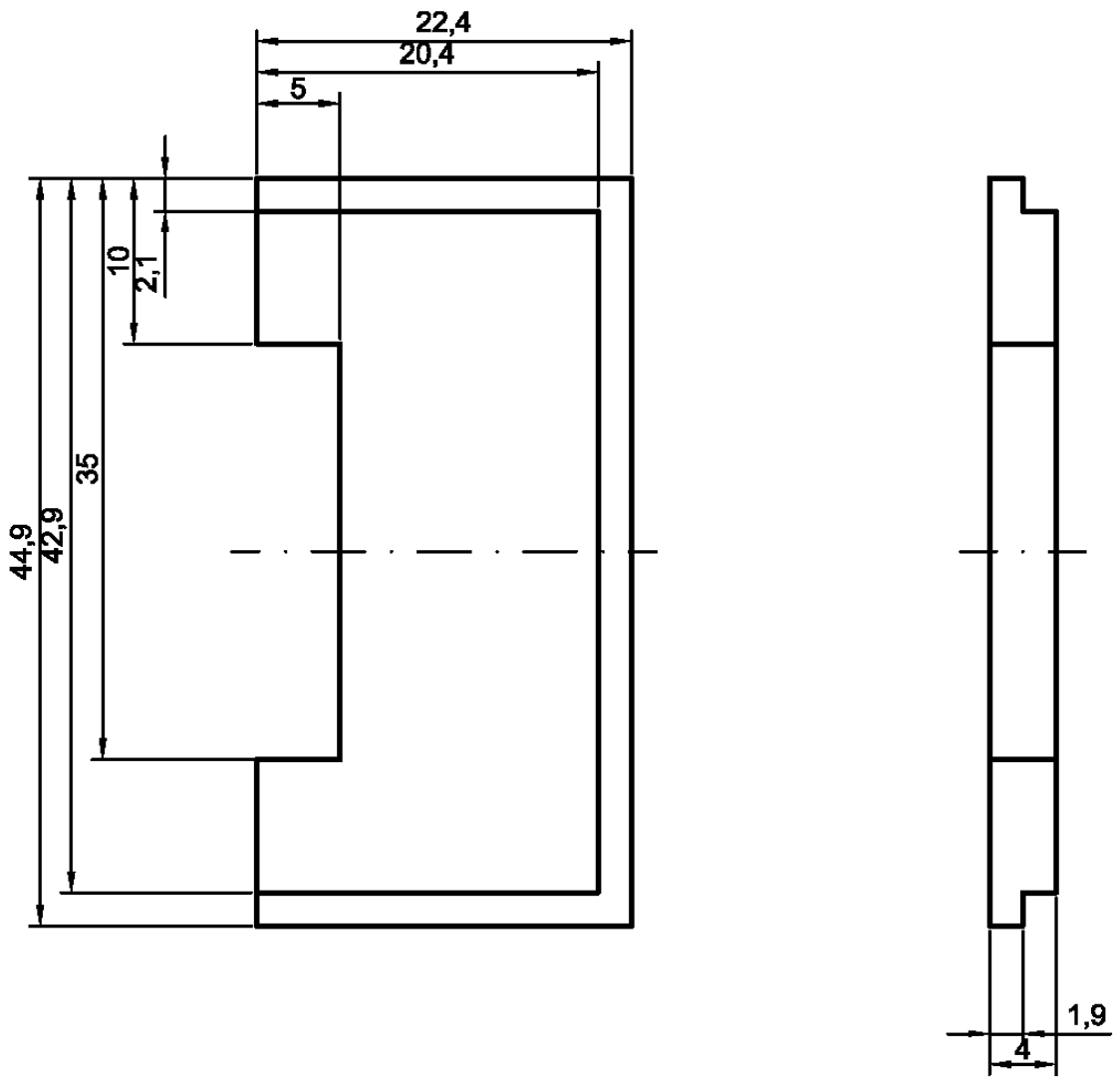

M 2:1

PC-Platte $4 \mathrm{~mm}$ klar 02 Stk.

\begin{tabular}{|c|c|c|c|c|c|}
\hline $\begin{array}{l}\text { Verantwort. Abtg. } \\
\text { NFG }\end{array}$ & $\begin{array}{c}\text { Technische Referenz } \\
860000\end{array}$ & $\begin{array}{l}\text { Erstellt durch } \\
\text { Y. LI; A. Grodrian }\end{array}$ & Genehmlgt von & $\begin{array}{l}\text { Datel Li03.dwg } \\
\end{array}$ & \\
\hline & & | Dokumentenart & ETZ & Dokumentanstatus & \\
\hline & & Tital, Zusä̈blicher Titel & & Bauteil-Nr. & \\
\hline & & Dec & & $\begin{array}{l}\text { And. Ausgabedgtum } \\
\text { 2015-12-02 }\end{array}$ & $\begin{array}{l}\text { Spr. } \\
\text { de }\end{array}$ \\
\hline
\end{tabular}



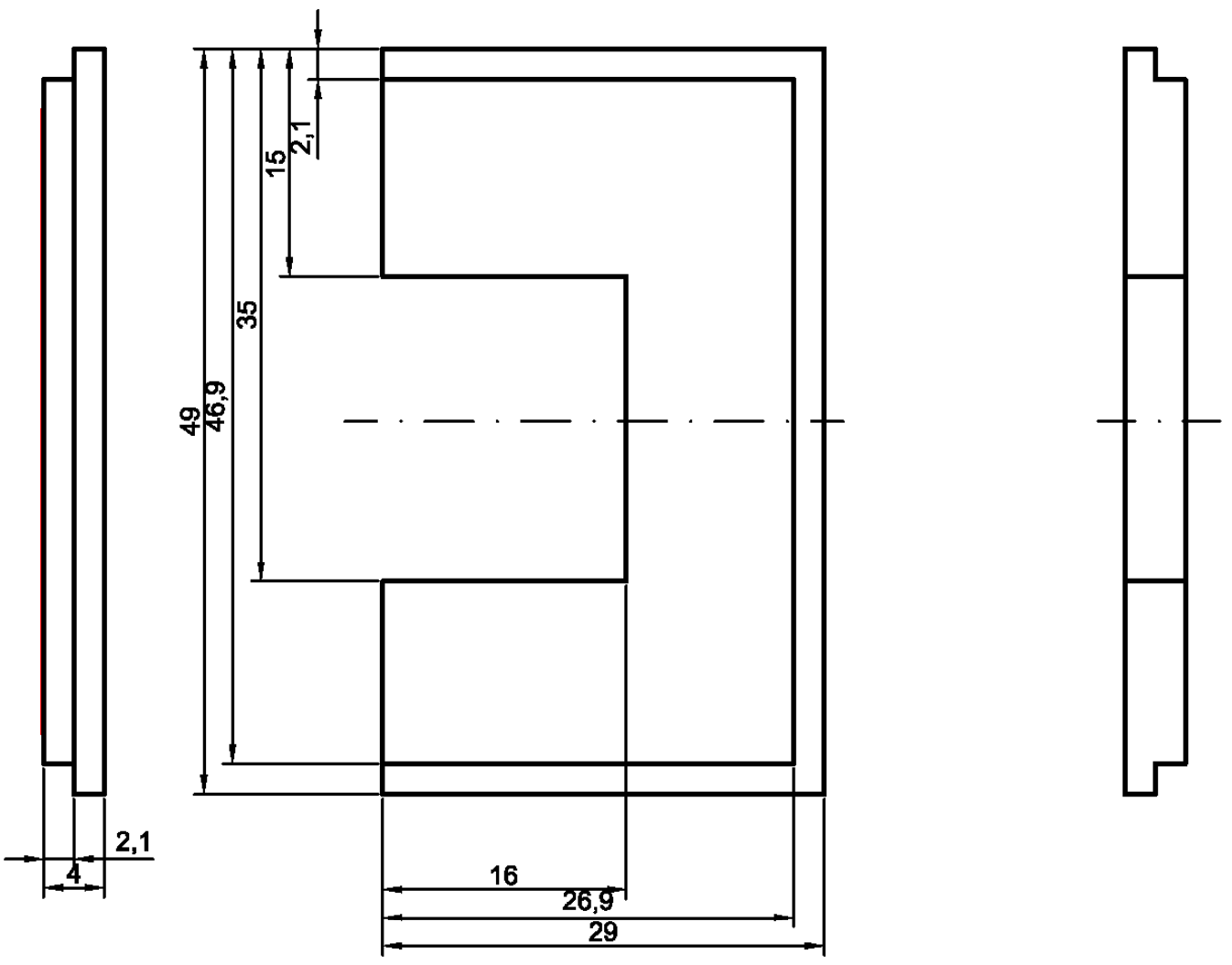

M 2:1

PC-Platte $4 \mathrm{~mm}$ klar 02 Stk.

\begin{tabular}{|c|c|c|c|c|c|}
\hline $\begin{array}{l}\text { Verantwortl. Abtg. } \\
\text { NFG }\end{array}$ & $\begin{array}{l}\text { Techniasche Reforenz } \\
860000\end{array}$ & $\begin{array}{l}\text { Errstallt durch } \\
\text { Y. Li; A. Grodrian }\end{array}$ & Genehmigt von & ${ }^{\text {Datel }}$ Li03.dwg & \\
\hline & & Dokumentenart & ETZ & Dokumentanstatus & \\
\hline & & \multicolumn{2}{|l|}{ Thtal, zusäatzlicher Tital } & Bauteil-Nr. & \\
\hline & & \multicolumn{2}{|c|}{ Seitenteil 1} & $\begin{array}{l}\text { And. Ausgabedatum } \\
\text { 2015-12-02 }\end{array}$ & Spr. \\
\hline
\end{tabular}



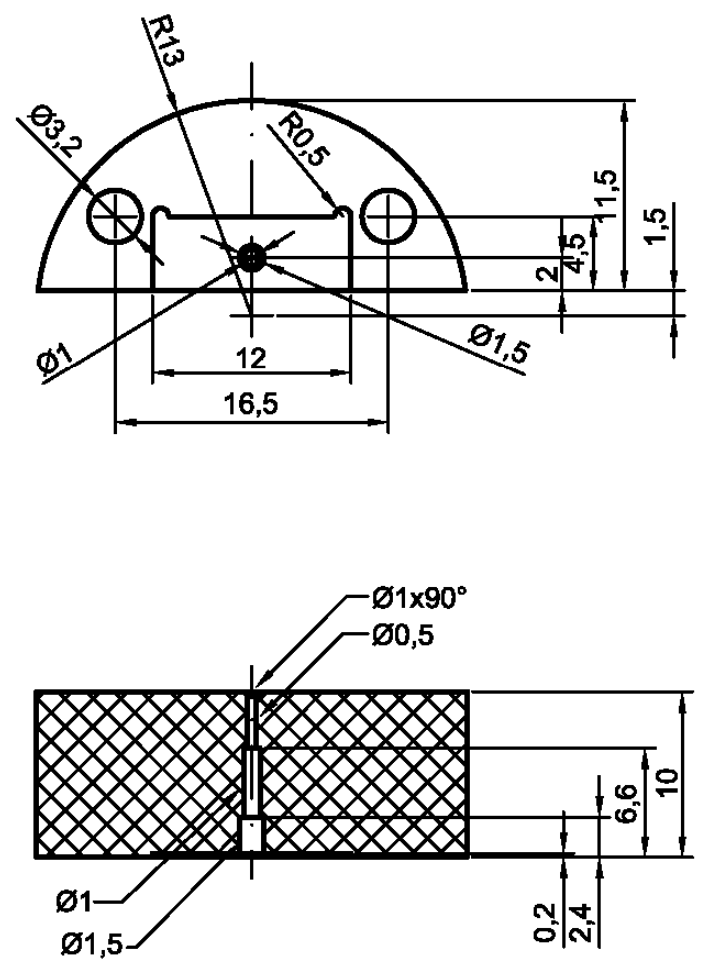

M 2:1

Rd 30 PC klar

01 Stk.

\begin{tabular}{|c|c|c|c|c|c|}
\hline $\begin{array}{l}\text { Verantwort. Abtg. } \\
\text { NFG }\end{array}$ & $\begin{array}{l}\text { Technische Referenz } \\
860000\end{array}$ & $\begin{array}{l}\text { Erstellt durch } \\
\text { Y. Li; A. Grodrian }\end{array}$ & Genehmlgt von & Diotel $\quad$ Liwg & \\
\hline & & \multicolumn{2}{|l|}{ Dokumentenart } & \multicolumn{2}{|l|}{ Dokumenttanstatus } \\
\hline & & \multicolumn{2}{|l|}{ Titel, zusä̈zlicher Titel } & Bauteil-Nr. & \\
\hline & & \multicolumn{2}{|c|}{ EWOD-Messzelle, Oberteil } & $\begin{array}{l}\text { And. } \\
\text { Ausggabedetum } \\
2014-09-01\end{array}$ & Spr. \\
\hline
\end{tabular}



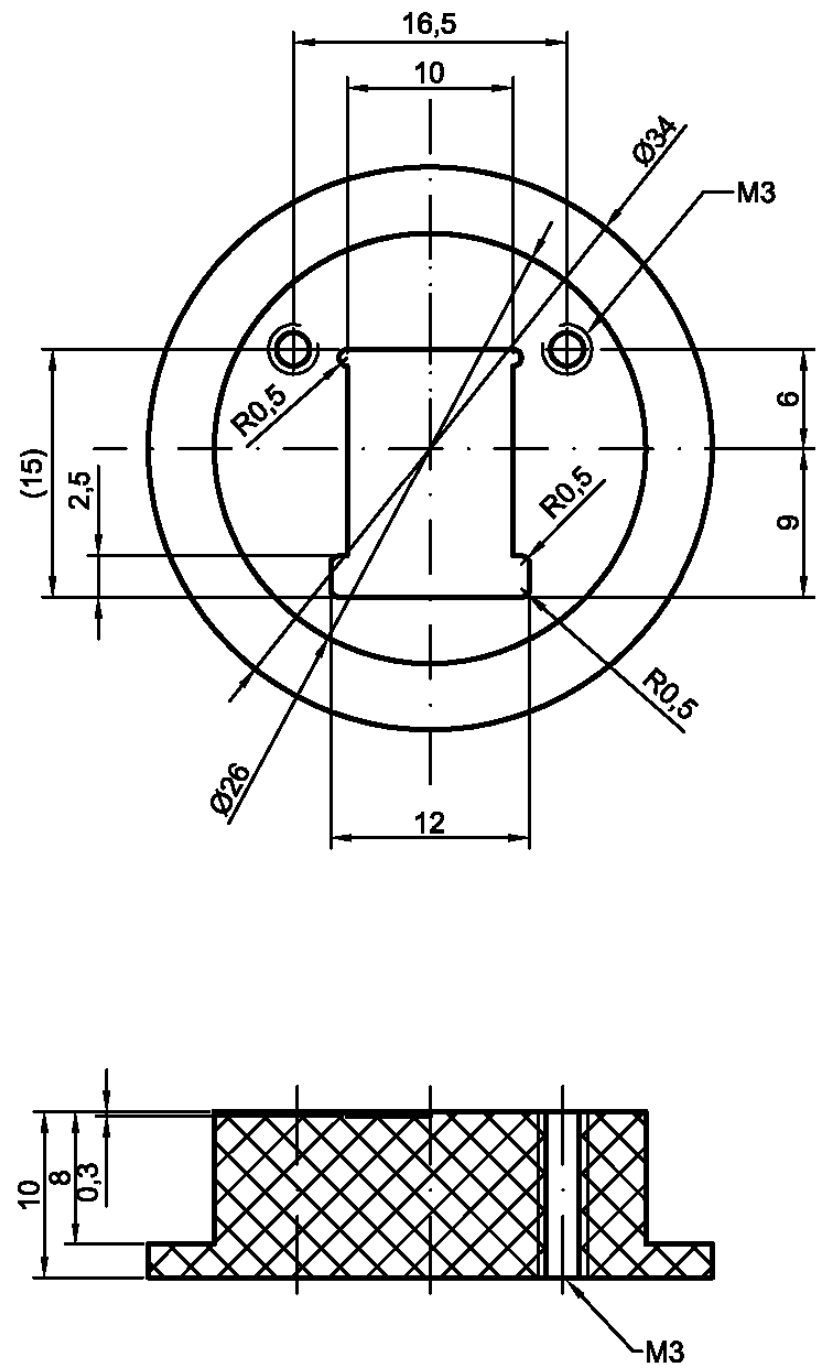

M 2:1

Rd 35 PC klar

01 Stk.

\begin{tabular}{|c|c|c|c|c|c|c|}
\hline $\begin{array}{c}\text { Verantwort. Abtlg. } \\
\text { NFG }\end{array}$ & $\begin{array}{l}\text { Technieche Referenz } \\
860000\end{array}$ & $\begin{array}{l}\text { Erstellt durch } \\
\text { Y. Li; A. Grodrian }\end{array}$ & Genehmlgt von & \multicolumn{3}{|l|}{ Li04.dwg } \\
\hline & & \multicolumn{2}{|l|}{ Dokumentenart } & \multicolumn{3}{|l|}{ Dokumentenstatus } \\
\hline & & \multicolumn{2}{|l|}{ Tital, zusätzlicher Titel } & \multicolumn{3}{|l|}{ Bauteil-Nr. } \\
\hline & & \multicolumn{2}{|c|}{ EWOD-Messzelle, Unterteil } & $\begin{array}{l}\text { And. Ausgabedetam } \\
\text { 2014-09-01 }\end{array}$ & $\begin{array}{l}\text { Spr. } \\
\text { de }\end{array}$ & \\
\hline
\end{tabular}



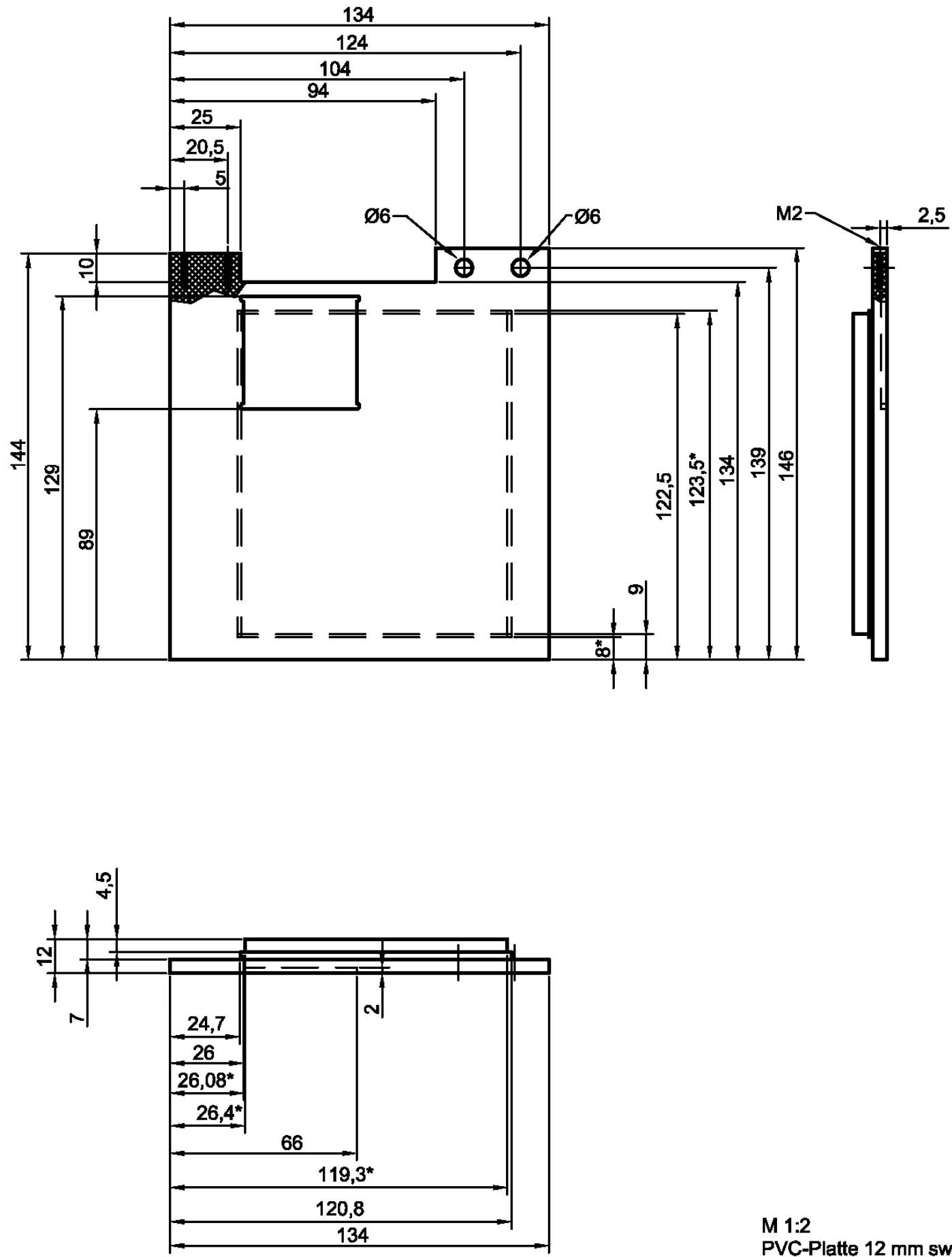

M 1:2

PVC-Platte $12 \mathrm{~mm}$ sw 01 Stk.

$\mathrm{XX}^{*}$ - anpassen!

\begin{tabular}{|c|c|c|c|c|c|c|}
\hline $\begin{array}{l}\text { Verantwortl. Abtg. } \\
\text { NFG }\end{array}$ & $\begin{array}{l}\text { Techniache Reforenzz } \\
860000\end{array}$ & $\begin{array}{l}\text { Erstollt durch } \\
\text { Y. Li; A. Grodrian }\end{array}$ & Genehmigt von & Li05.dwg & & \\
\hline & & \multicolumn{2}{|l|}{ Dokumentenart } & \multicolumn{3}{|l|}{ Dokumentanstatus } \\
\hline & & \multicolumn{2}{|l|}{ Tital, Zusä̈alzlicher Tital } & \multicolumn{3}{|l|}{ Bauteil-Nr. } \\
\hline & & \multicolumn{2}{|c|}{ Mikroskoptischdecke } & \begin{tabular}{l|l} 
And. & Ausgabedatum \\
$2014-09-01$
\end{tabular} & $\begin{array}{l}\text { Spr. } \\
\text { de }\end{array}$ & Blatt \\
\hline
\end{tabular}




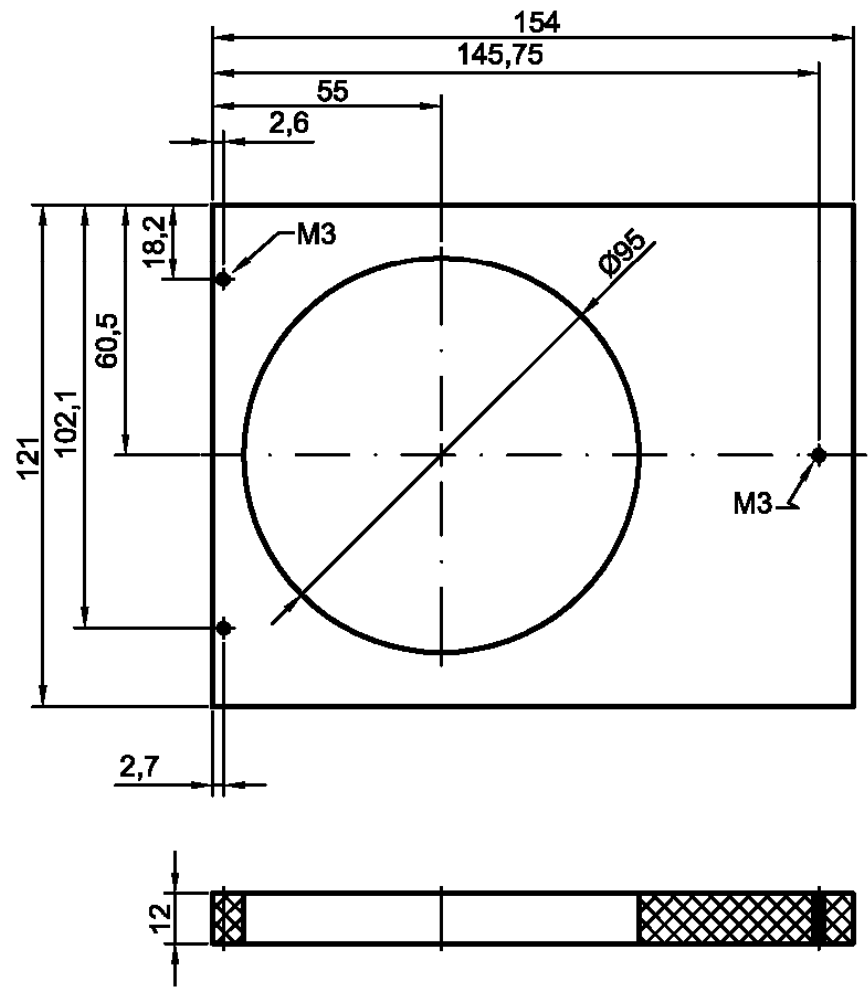

Befestigungsbohrungen $\varnothing 4$ auf Laborboy (Hebebühne) nach Muster

M 1:2

PVC-Platte sw, $12 \mathrm{~mm}$ anreißen und bohren

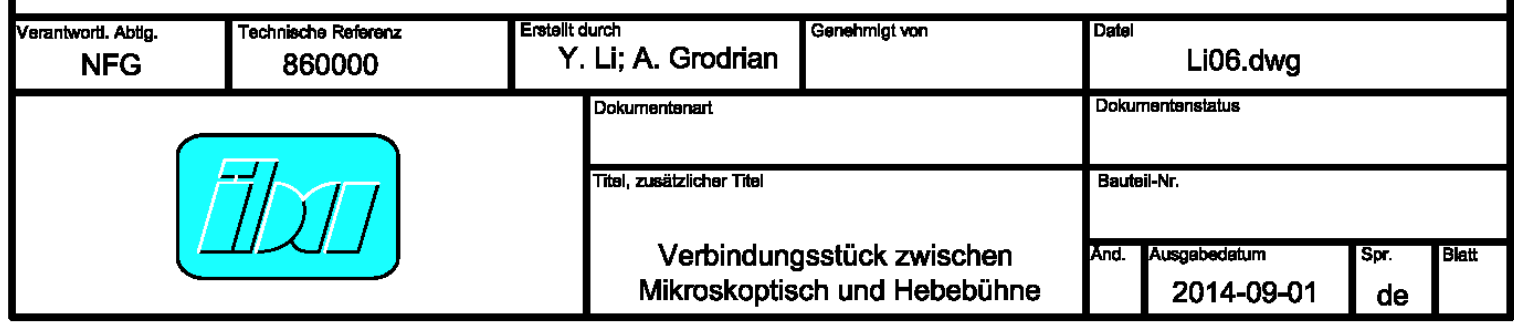



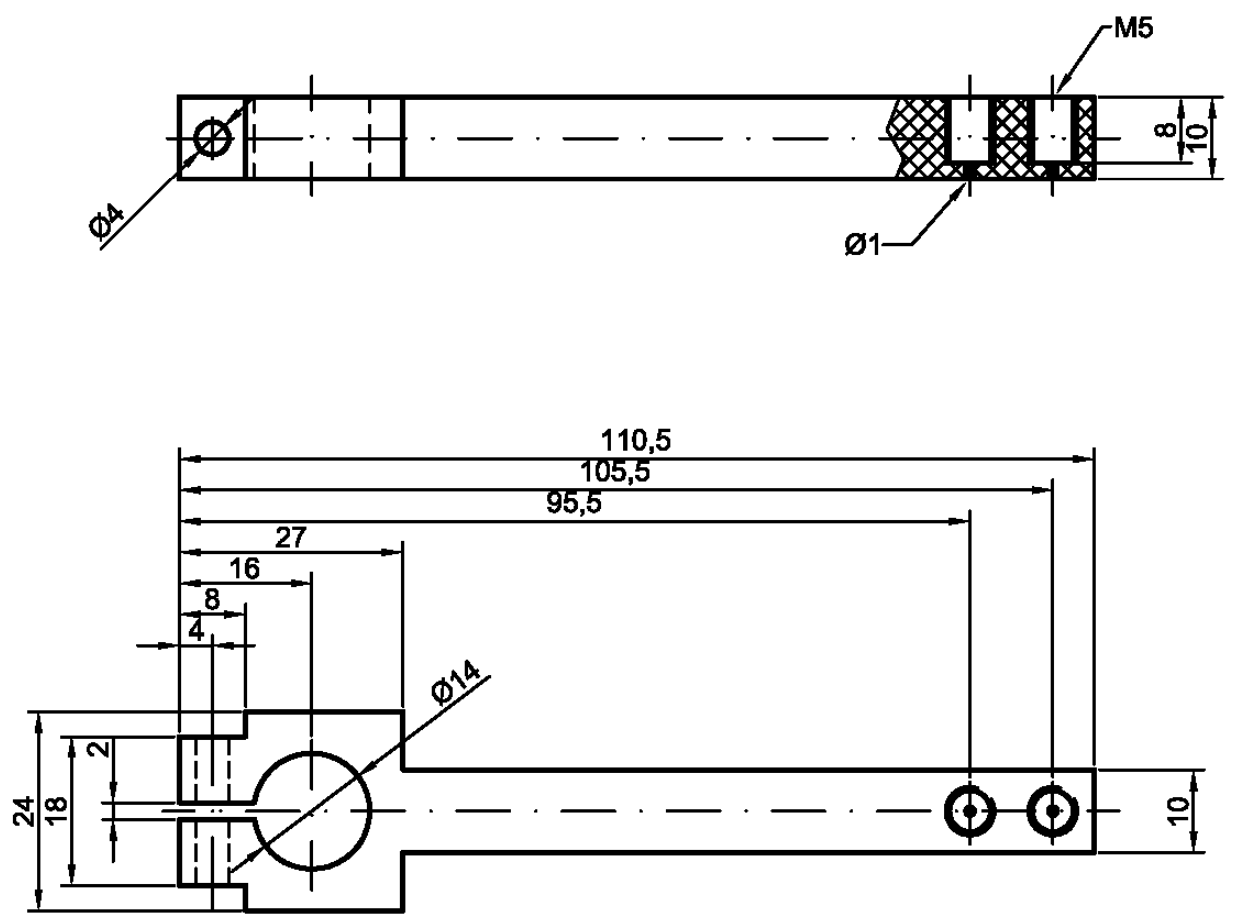

M 1:1

PVC-Platte $10 \mathrm{~mm}$ gr 01 Stk.

\begin{tabular}{|c|c|c|c|c|c|}
\hline $\begin{array}{l}\text { Verantwortl. Abtlg. } \\
\text { NFG }\end{array}$ & $\begin{array}{c}\text { Technische Referenz } \\
860000\end{array}$ & $\begin{array}{l}\text { Erstollt durch } \\
\text { Y. Li; A. Grodrian }\end{array}$ & Genehmlgt von & Li07.dwg & \\
\hline & & \multicolumn{2}{|l|}{ Dokumentenart } & \multicolumn{2}{|l|}{ Dokumentenstatus } \\
\hline & & \multicolumn{2}{|l|}{ Trtal, assäblalicher Thtel } & \multicolumn{2}{|l|}{ Bauteil-Nr. } \\
\hline & & \multicolumn{2}{|l|}{ Balken } & $\begin{array}{l}\text { Alusgobededam } \\
\text { 2014-09-01 }\end{array}$ & $\begin{array}{l}\text { Spr. } \\
\text { de }\end{array}$ \\
\hline
\end{tabular}




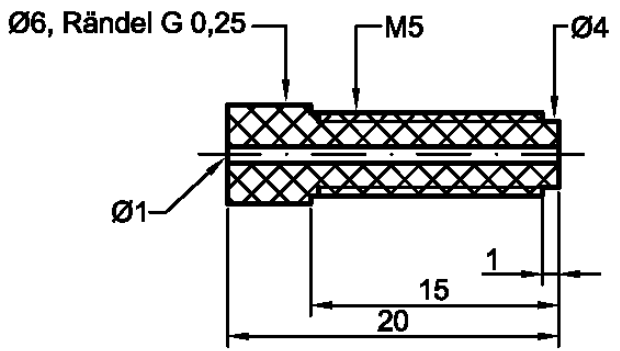

M 2:1

Rd 6 PEEK

01 Stk.

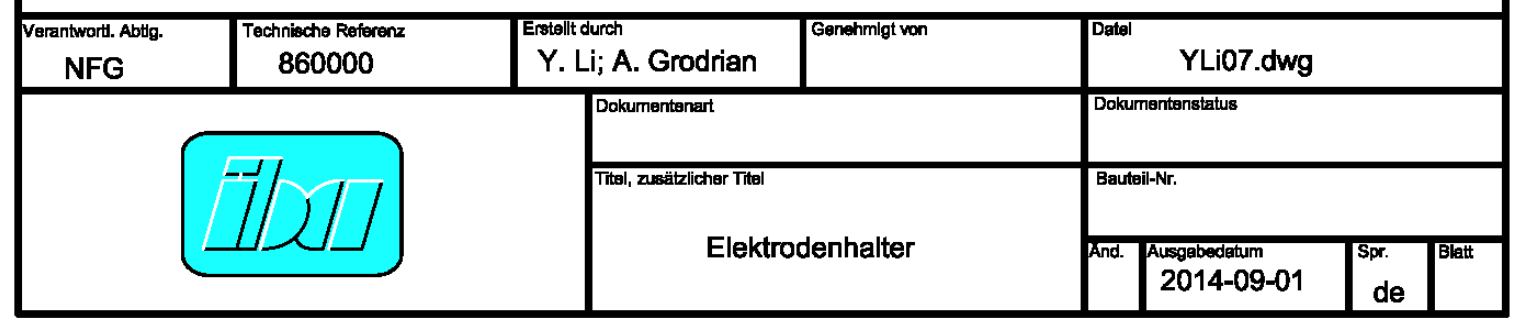



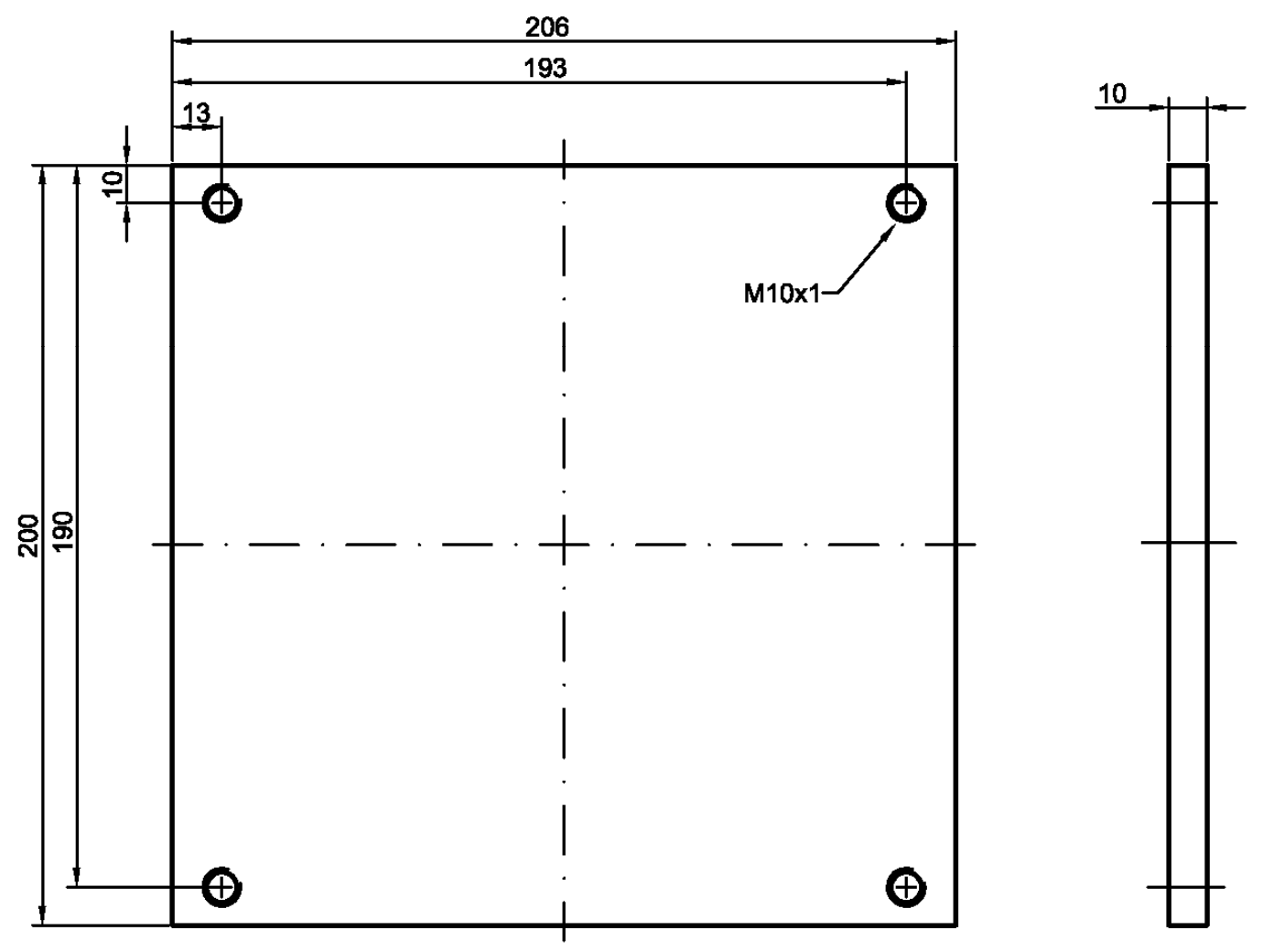

M 1:2

BI 10 AlMgSi0.5 F22

01 Stk.

\begin{tabular}{|c|c|c|c|c|c|}
\hline $\begin{array}{l}\text { Verantwortl. Abtg. } \\
\text { NFG }\end{array}$ & $\begin{array}{l}\text { Technische Reforenz } \\
860000\end{array}$ & $\begin{array}{l}\text { Erstallt durch } \\
\text { Y. LI; A. Grodrian }\end{array}$ & Genehmlgt von & \multicolumn{2}{|l|}{ Li08.dwg } \\
\hline & & \multicolumn{2}{|l|}{ Dokumentenart } & \multicolumn{2}{|l|}{ Dokumenttenstatus } \\
\hline & & \multicolumn{2}{|l|}{ Trtal, avsäzlalicher Thtel } & \multicolumn{2}{|l|}{ Bauteil-Nr. } \\
\hline & & \multicolumn{2}{|c|}{ Stützplatte } & \begin{tabular}{l|} 
And. \\
Ausgabededum \\
2014-09-01
\end{tabular} & $\begin{array}{l}\text { Spr. } \\
\text { de }\end{array}$ \\
\hline
\end{tabular}



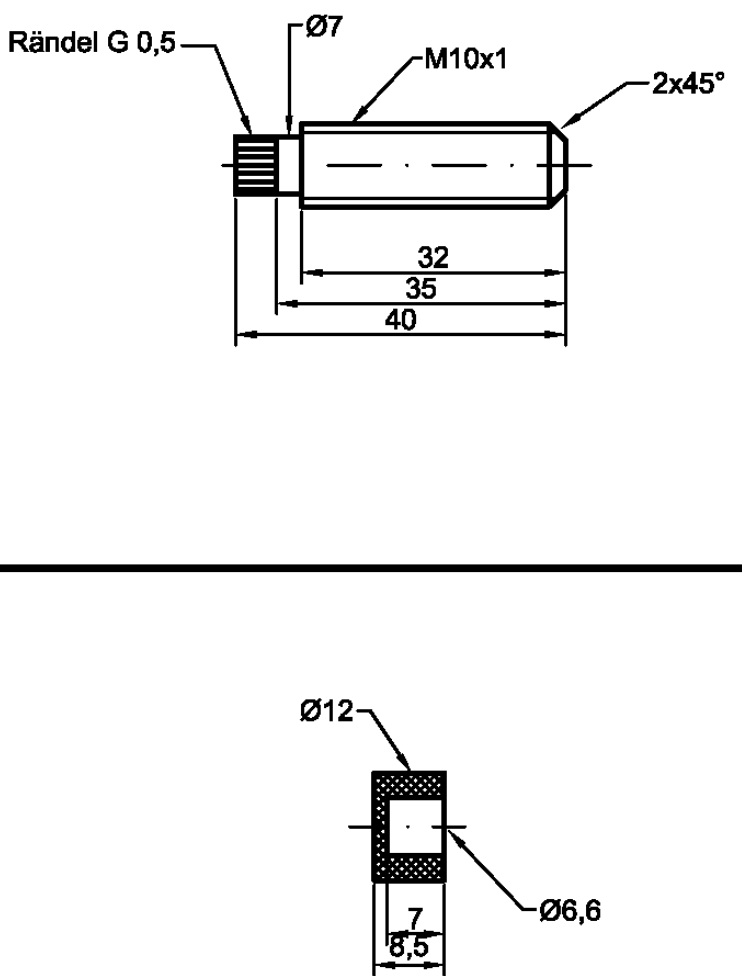

M 1:1

Rd 12 PVC gr

04 Stk.

Drehknopf

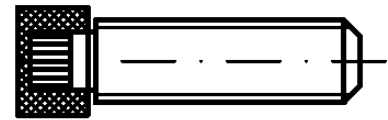

Drehknopf und Schraubengewinde verpressen !

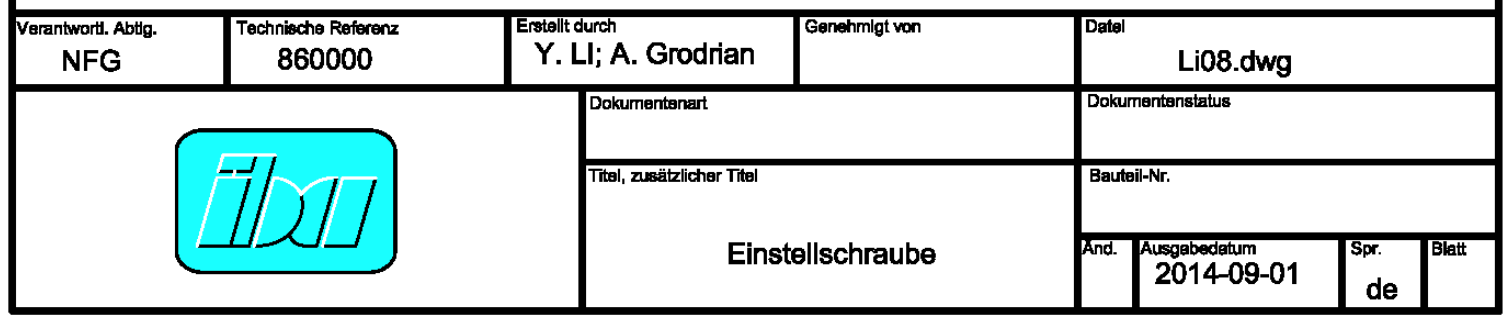




\section{Publication}

$10 / 2017$

Y.-J. Li \& B. P. Cahill „Frequency Dependence of Low-Voltage Electrowetting Investigated by Impedance Spectroscopy", Langmuir 2017, 33, 13139-13147.

\section{Selected Conference Contributions}

$09 / 2017$

$06 / 2017$

$03 / 2017$

$09 / 2016$

09/2016

$04 / 2016$

$02 / 2016$

$09 / 2015$

$03 / 2015$
Y. Li \& B. P. Cahill „Investigation of Low-voltage Electrowetting on Multilayer-Dielectrics with Impedance Spectroscopy", $12^{\text {th }}$ International Symposium on Electrokinetics (ELKIN) 2017, Dresden (rapid fire presentation \& poster).

Y. Li \& B. P. Cahill "Low-Voltage Electrowetting on Dielectric (EWOD)", $16^{\text {th }}$ European Student Colloid Conference (ESC) 2017, Florence (oral presentation).

Y. Li \& B. P. Cahill "Impedimetric Analysis of Low-Potential Electrowetting Process", 11. Interdisziplinäres Doktorandenseminar 2017, Berlin (oral presentation).

Y. Li \& B. P. Cahill „Impedimetric Measurement of Low Potential Electrowetting on Dielectric (EWOD)“, $9^{\text {th }}$ International Workshop on Impedance Spectroscopy (IWIS) 2016, Chemnitz (oral presentation).

Y. Li \& B. P. Cahill "Impedimetric Measurement of Low-Voltage Electrowetting on Dielectric", 7. Kurt-Schwabe-Symposium 2016, Mittweida (oral presentation).

Y. Li \& B. P. Cahill "Low Potential Electrowetting on Dielectric Based on Tantalum Pentoxide and Silane", $12^{\text {th }}$ Zsigmondy Colloquium 2016, Saarbrücken (oral presentation).

Y. Li \& B. P. Cahill "Low Potential Electrowetting System", $8^{\text {th }}$ Chemical and Biological Micro Laboratory Technology 2016, Elgersburg (poster).

Y. Li \& B. P. Cahill, „Niedrigspannungs-Elektrowetting-System basierend auf anodisiertem $\mathrm{Ta}_{2} \mathrm{O}_{5}$ und Silanen“, 11. Thementage Grenz- und Oberflächentechnik 2015, Zeulenroda (oral presentation).

Y. Li \& B. P. Cahill „Niedrigspannung-Elektrowetting“ 9. Deutsches Biosensor Symposium 2015, München (poster). 


\section{DANKSAGUNG}

An dieser Stelle möchte ich mich bei vielen Menschen herzlich bedanken, die mich auf dem Weg der Promotion begleitet und unterstützt haben.

Dr. Brian Cahill danke ich für die spannende interdisziplinäre Themenstellung und für die Möglichkeit eine Promotion in seiner Nachwuchsforschergruppe am Institut für Bioprozessund Analysenmesstechnik e.V. in Heiligenstadt (IBA) durchzuführen. Außerdem danke ich inm für die Unterstützung, mich in verschiedenen Bereichen weiterbilden zu lassen.

Prof. Andreas Janshoff danke ich für die Übernahme der Betreuung und Begutachtung von Seiten der Universität Göttingen, für alles Feedback und alle Diskussionen sowie für seine Unterstützung und Hilfe seit meiner Masterarbeit in seinem Arbeitskreis.

Dem weiteren Mitglied des Thesis-Komitees, Prof. Philipp Vana, danke ich für die Betreuung, die Übernahme des Korreferats, die hilfreichen Ratschläge und Diskussionen bei meinen jährlichen Berichten und das Verständnis bei der Terminfindung.

Ich danke den weiteren Mitgliedern der Prüfungskommission, Prof. Sarah Köster, Dr. Oliver Bäumchen und Dr. Thomas Burg.

Aufgrund der Interdisziplinarität meines Forschungsthemas bin ich bei vielen Kollegen in allen Abteilungen der IBA (Analysenmesstechnik, Bioprozesstechnik, Biowerkstoffe, Werkstatt und Verwaltung) zum Dank verpflichtet. Ohne deren Hilfe und Unterstützung wäre diese Arbeit nicht zustande gekommen:

Prof. Uwe Pliquett danke ich für die gute Kooperation, Unterstützung und Begutachtung bei der Datenauswertung der dynamischen Impedanzspektroskopie, für die hilfreichen Diskussionen und für die große Gastfreundschaft der Familie Pliquett.

Danny Echtermeyer und Jiaji Pan danke ich für die Entwicklung und Bereitstellung von Hardware und Software bezüglich der dynamischen Impedanzspektroskopie und Hilfe beim Verständnis über Elektrotechnik und Signalverarbeitung.

Andreas Bartel danke ich für die Bereitstellung der Hardware für die Impedanzspektroskopie im Frequenzbereich.

Dr. Marion Frant, Holger Rothe und Johanna Ludolph danke ich für die fachkundige Unterstützung bei Kontaktwinkelhysterese-Messungen und bei AFM.

Peggy Reich danke ich für die wertvolle Unterstützung und Diskussionen bei Impedanzspektroskopie und Zyklo-Voltammetrie sowie für die nicht-fachliche Bereicherungen.

Dr. Viktor Schroeder und Dr. Jörg Schemberg danke ich für das sorgfältige Korrekturlesen meiner Dissertation, konstruktive Diskussionen und die vielen hilfreichen Tipps.

Andreas Grodrian danke ich für die inspirierenden Diskussionen und Ratschläge über die Konstruktionskonzepte verschiedener Bauteile meines Messaufbaus und für die elektronische 
Umwandlung meiner Handzeichnungen. Der Werkstatt danke ich für die große Hilfsbereitschaft und die hoch-präzise Fertigung der Bauteile. Daniel Martin danke ich für die Einführung in das Zeichnungsprogramm Inventor.

Robert Römer danke ich für die Bereitstellung des Labview-Programs, für die tollen Ratschläge für Mikroskopie und Photographie.

Annerose Lindenbauer danke ich für ihre Unterstützung im Labor.

Prof. Dieter Beckmann, Ulrich Marschall und Sebastian Kaufhold danke ich für die Unterstützung von Seiten der Verwaltung.

Bei vielen Kollegen am IBA, die ich nicht erwähnt habe, danke ich für die Hilfsbereitschaft, das angenehme Arbeitsklima und die schöne Zeit.

Für die Unterstützungen bei meiner experimentellen Arbeit an der Uni Göttingen sowie für die organisatorische Hilfestellung danke ich den Mitgliedern der Arbeitsgruppe Janshoff: Helen Nöding, Hannes Witt, Marieelen Oelkers, Marian Vache, Sarah Verbeek, Jonathan Bodenschatz, Andrea Cordes, Elena Polo und Petra Lappe.

Dr. Bernhard Schröder danke ich für die große Unterstützung als Mentor, für die Motivation und konstruktive Ratschläge.

Sten Appelt und Gisela Wagner danke ich für die Freundschaft, aufbauende Worte und für leckeres Essen sonntagmittags.

Meinen Freunden aus dem Malzirkel danke ich für die schönen Stunden, gemeinsam in die künstliche Welt einzutauchen und die Alltäglichen mit anderen Augen zu betrachten.

Meinen Eltern (Shihua Pan und Zhengming Li) gilt mein besonderer Dank für den Rückhalt, die aufbauenden Worte und die Unterstützungen während der Doktorarbeit besonders in schwerer Zeit.

在此, 我特别感谢我的父母 (李正明与潘世华)一直以来的支持与鼓励, 尤其是他们在我读博最 艰难的时候对我的关爱与勉励! 